IV.

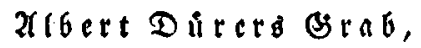

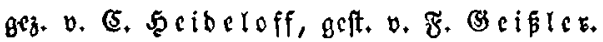

Ábert Dürer, (Sohn Zabert Ditrerz b. a. eines bejdidten Bolbjdmibtz, ber auz Evtab, einem Dorfe in SVer $=\mathfrak{u n g a n n}$ unweit bez Flecent

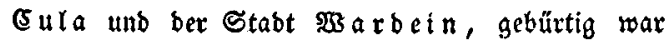
und fich 1455 nad) Rürnberg gewanot batte, ) warb geb. zu $\mathfrak{x}$ ürnberg am 20. Mai 1471, ftarb bas. felbft, 57 Sabr alt, am 6. 2fpril 1528 unb warb auf Dem S.t. Sobannis Frteshafe beerbigt. ${ }^{*}$ ) uriprüng=

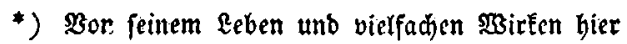
cin Metrererz anzuführen, verbeut bie Beforänkts Geit bez Raumez. Rur eine georängte Gharakterie ftie mag hier \$plab finden, ba fie unb cben fo wabe

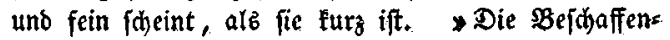
beit feiner Geele war lebbaft und bebacitjam, feu= rig unb anmuthig, ftark uno bequem; feine Einbile

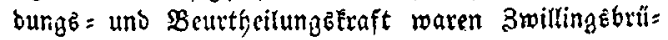


Xx

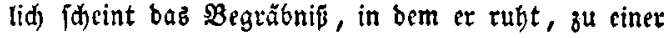
Bruft ber Familie $\mathfrak{F} r \mathrm{i}$ beftimmt gerejen zu fenn. Denn auf ber Mitte ber grojen Stcinplatte, weltie cs bebect, ift bie Infdurift:

\section{CCCC. XXt.}

Ser . Freben . Begrebnis .

nebft zrori গুJappenjdilbern eingehauen, bie $n i d t$ Dürers fino, Das cine berfelben hat vier Felber, nämlia cinanoer fdräg gegenübergeftellt zroei blaue Felder, und zwei nit jogenannten Eijentütlein ober benjelben ätnlithen Figuren; bas anbere zroei fitberne

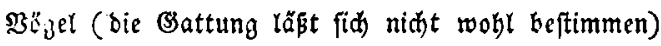
im blauen folbe. Därerz Shitbzeid)en bingegen wae ein rebendes uno ftellte eine Shür vor; wie man benn vor arters bie Garten und weidjen Eonjonanten wenig unterifieb, und, um cin ganz nabe liegeroses Beippiel anzuführen, felb/t in urfunben eben fo gut

ber, beren ber erftgeborne einen fleinen $\mathfrak{B}$ orzug vor bem andern batte.". So jagt $\mathfrak{g}$ e $i \mathfrak{n}$. So n $r$. ' $x$ r e $n$ im: (Sebähtnís ber Egren albert Dürers. (5)ßlar, 1728. Hl. 8. 
Düringen, ols Thüringen fdrieb. Uuth follen ifym vom Raifer Maximilian I. - wie man erzăglt,

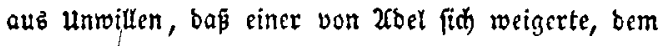
eblen Meifter bie wankenbe Leiter zu balten - bod viculeidt nicht ifm allein, fonbern ber ganzen löbli= hen Malerzuft, bret fitberne Sdilblein im azurnen Eerbe, zwei oben und eins unten, varticben worben (ev). *) So fojenEte Raifar Fricbríd III. Dem Sobann $\mathfrak{R}$ äntelin unb ben gejammien $\mathfrak{B u t h}=$ bruderen im כahr 1458 sinen getrönten Eümen zum Innungejoiltbe.

Soldhergeftalt gehörte gedahtę Segräbniß fon bet Bivcifel ber Familie von Dürers Sđyregervater an. Dürciz Frau, mit welder er fid) leiber! fofon im Sabr 1494 verchelidte - igre unaufbürlidien

*) In einer untriflifift zu Dürere Sitbe, wetde Sanbraxt $\mathfrak{2 h}$. 1. S. 225. nnfübut, beipt ez:

Contulit hu i c Caesar insignia nobilitatis:

Tantus honos artis famaque tan'a viri!

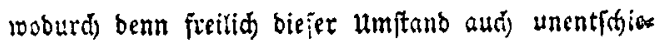
ben bleibt. 
XXII

Suälereien jollen nämlid), nadh \$irtheimers unb an= berer glaubwüroiger Mämner 3eugnifle, fogar ben Rebenzfaben bez Gerrtiden Rünftlerz yor ber 3cit

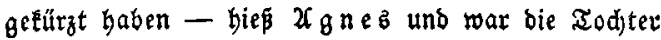

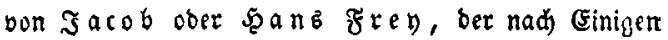
sin berübmter Med)anicuz, nać) Jndern sin guter Sänger und Şarfenjpieler gewejen fegn joll. $\mathfrak{B e r}=$ muthlidy war ex beibes und wohl nod mandies bane=

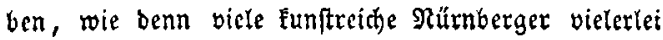
zugleidy auf̧ rübmlid)fte übten.

Die metallne Brabjdrift, weldye in ben fteincr=

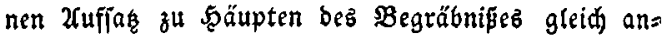
fünglidi eingelegt worben ift, lautet alfo:

Me, Al. Du.

Quidqnid Alberti Dureri mortale fuit, hoc couditur tumulo; emigravit VIII. Id. April. MDXXVIll.

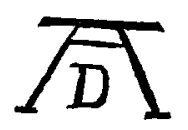




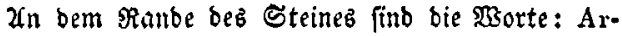
chitecturn. Piotura. Sculptura, eingebauen roorden.

Zlber bem gropherzigen Fünftler uno אünfters freunbe $\Im$ oadim bon $S_{a n d} \mathfrak{a r t}$ gnügte baran nidyt, jonbern ex lies im Jabr 1681 bas Brabmal erneuern und eine gegoffene Metallptatte mit zwei Jniđuriften beifügen, nämlid) redte :

Vixit Gernaniae suae decus

Albertus Durerus

Artiun lumen Sol artificum

Uıbis patriae Nor. oruameutum

Piclor Chalcographus Sculptor

Sine exemplo quia omníscius

Diguus inventus exteris

Quem imitandam censerent

Magmes magunturn Cos ingeniorum

Post scsqui seculi requiem

Quia parem non habuit

Solus heic cubare jubetur.

Tu sparge flores Viator.

A. R. S. MDCLXXXI.

I. de $S$, 


\title{
xxIV
}

und lints :

\author{
Sier rube \\ ภunftler Fürft! \\ Du mehr als groper Mann! \\ Snn biel Runft hat es bir \\ Rod feiner gleid gethan. \\ Die Ero war ausgemalt, \\ Der Simmel did jekt hat; \\ Du maleft beilig nun \\ Dort an Der Botteżtadt. \\ Die

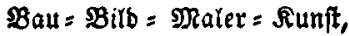 \\ Die nenmen bich spatron \\ unb jegen bir nunauf \\ im $\mathfrak{Y} 0 \mathrm{~b}$ \\ bie \\ Eorber $=$ Aron.
}

Dic bartnter aingehauene 3ahl 649. ift bie Rummer bes Segräsniftez; aud fteht nod am Ranbe zull ben Füpen: Rero. A. 1681 .

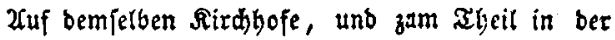
Rähe von Dürero Grabe, befinden fid) nod wehtere 


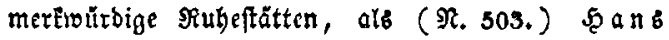

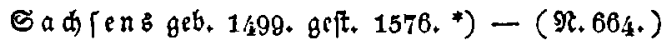

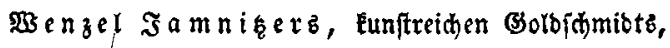
Email = Malers, Rupferftedsers uno Formidneibers, geft. 1585. - ( $R, 1.14$. unweit ber Selziduther=

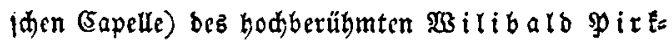
beimers, ber Dürers treuefter Freund war, geb. 1470. geft. 1530. - enblic) (auf bem fogenannten

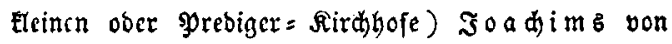
Sanbrart, geb. 12. Mai 1600ิ. geit. 1688.

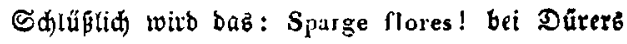
Brabjüăte noch immer auf baz beiligfte befolgt, wie folgenoe, unz von befreunocter sanb mitgetheilte vaduritibt bejagt:

*) Sonberbar genug crtäbnt bie Srabfarift nut

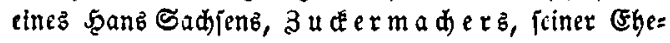
within und ifren beiben Erben. Sie 3abrzabl if

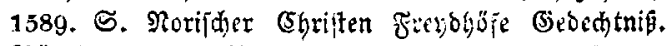
9ürnberg 1632. S. 76. - Drr ehrbare 3ెtderma= d)cr! Sollte or fich Des Shubmadicte unb poetcn gefdämt haben, obue ben folbft feines ఇamen Rie: mano geoädite? 
XXYI

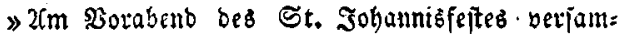
meln fid jebesmal fämmtlithe 2 feabemiler unb andere

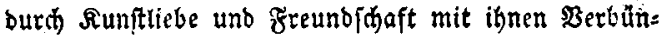
bete auf ber Nürnbürger $B$ Burg, im Rocal ber SRtuts tex E $x \mathfrak{n} \mathfrak{f}$, einer bejahrten, febr wadkern und lebenz: beitern Matrone, weldje biz Beföftigung metrerer von ben 2 fardemifern beforgt. Fränze aus (Fin)en= laub und Blumen werben gemeinidaftlich gewunoen und im 2(uztaujde freunsjofittider (Bejpräd)e, unter Erinnerungen an ben gropen. Sobten, unter 206fine

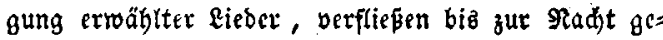
nupreid) bie Stunden. Mit 2 nbrudte bez folgenden Dorgenz finben fid $\mathfrak{Z}$ lle auf ber jogenannten $\mathfrak{F} r$ eiung (einen großin Borplaz̧e ber Burg, welder ben B̈pt=

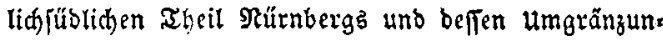
zungen in herridjer Milchung über(d)auen läpt) feft= lid gefteibet wieber ein, unb begeben fid in feierlie d) 3uge nad) bem St. Iogannizfriebgofe, wojetbft Dürers ajhe nun foft feit 300 Jabren ruht. ${ }^{*}$ ) Die Grabftätte wirb mit ben am vorigen 2 benbe gerwun=

*) Die Säcular = Frier am 6. Xpril 1828. wirb bas 


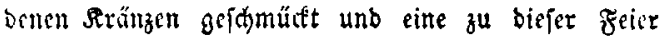
sigens gebidtete Şymme im Ehor abgefungen, fobann

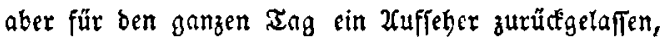

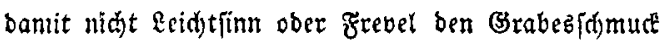
bes Rünftrerfïrften ftöre, ben Rürnberg mit gerchì: tem Stolze ben Seinen nenut.

Der für 1823*) von cinem uns niđjt belannt morbenen Sarfaffer gebiditete (Sejang lautet aljo:

Stärtenbe Morgenluft

शisebt um bie bunfle Bruft,

Die $\mathfrak{D}$ id umfdieft.

Eoler, Bollendeter, eqertidifer, lieblider, Reid a a beftat teter

Reifter ber Runft. -

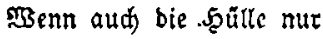
Ruthte, bie Eleinfte Spur

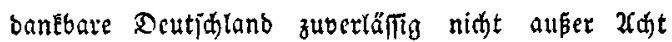
laffen! SBa er am 7. Mat (Dürets Beburtatage) 1771. getarten warb, ift uns unbelannt.

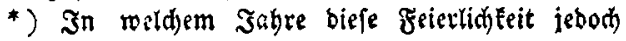


Răngit ift bahin;

Jmmer im şseltgewühl

Sey ez unz cin $\mathfrak{a} \tilde{y})$,

Ernjt wirb bes Eebens Spiel

xn biefer Eruft.

Reunmal fdon fand dis ascit

Bor ber unenolidgét,

כtarb aub die $\mathfrak{B}$ belt,

Seit $\mathfrak{D} i$ h bie Fube fand!

Sroṕ wirft $\mathfrak{D}$ u nod) genumnt,

Rid)t nur im Baterland -

$\mathfrak{B S} \circ$ man $\mathfrak{S} i d$ nennt.

unb bie fum Feltgericht

Strablet bafflbe $\mathfrak{i}$ d $\mathbf{t}$

Slorien = Slanz

um $\mathfrak{D}$ e i n veritärteb Bilb,

$\mathfrak{D}$ a $z$ rein unb Elar unb mils

nach ber Xngabe auf bem gernucten (3ebight am 2a.

Juni ftatt gefunden zu baben forint. 
รrroifdes Sebnen ftillt,

$\mathfrak{B i e} \mathfrak{D} \mathfrak{u}$ einft waxft.

1 Raphactz Sinnitz

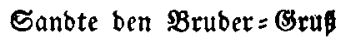

Einft $\mathfrak{D}$ i $\mathfrak{r}$ zurüd.

\$3as fich auf Erben fanb,

CFilt längft fhon şand in Şanb

Im böbern sünftlex $=$ \&and

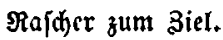

Stolz un[rer Baterftabt -

Mübe unb lebenafatt

Sankft $\mathfrak{D}$ u babin,

Dulbeteft, Yitteft viel,

\$3arft übler Eaunen Spiel,

Raftloz bod bin fum siel

Strebte $\mathfrak{D}$ ein Seift.

(Slübender Farbenton

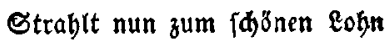

Ringz um $\mathfrak{D} i$ d her,

গ̧ą $\mathfrak{D}$ u auf fidhrer Spur 
$\operatorname{xxx}$

Zuncteft, hontef nux,

Siebfi $\mathfrak{D} u$ in ber giatur

5̧öberer Milt.

Bater, wir bringen hier Blumen und Thrî́nen $\mathfrak{D} i$ Sey) ftete in uns. Ebler- Bollendeter terrtider-lieblider, $\Re$ eid a us geftatteter Meifer ber $\mathfrak{A} u n$ It! Der Seraubeber. 


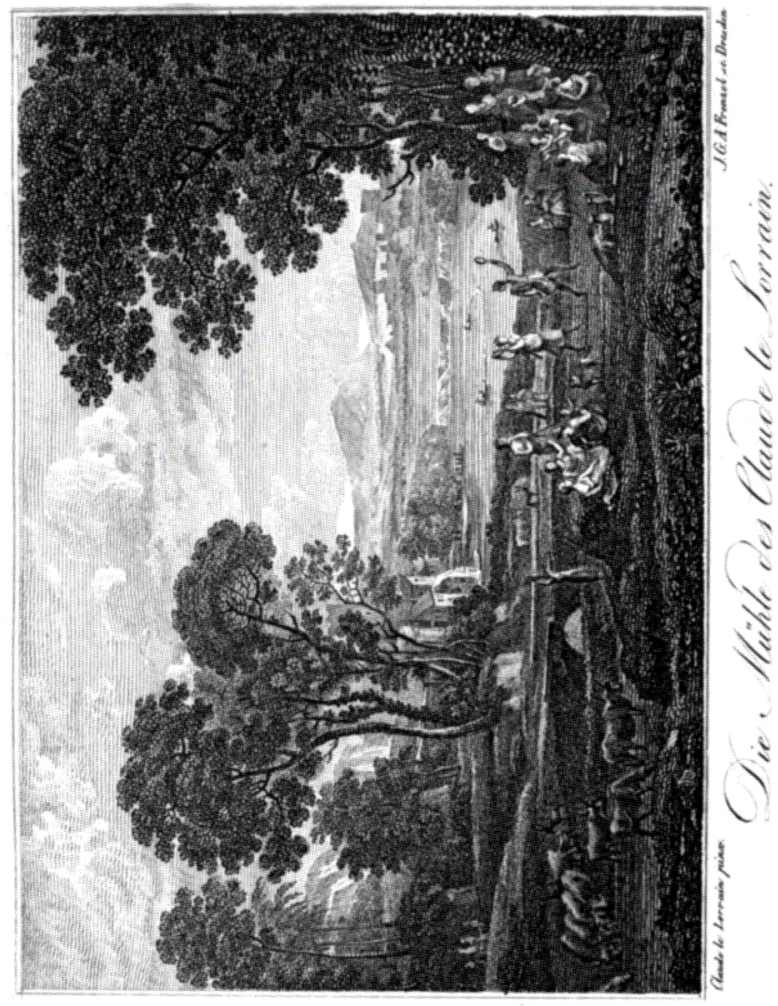





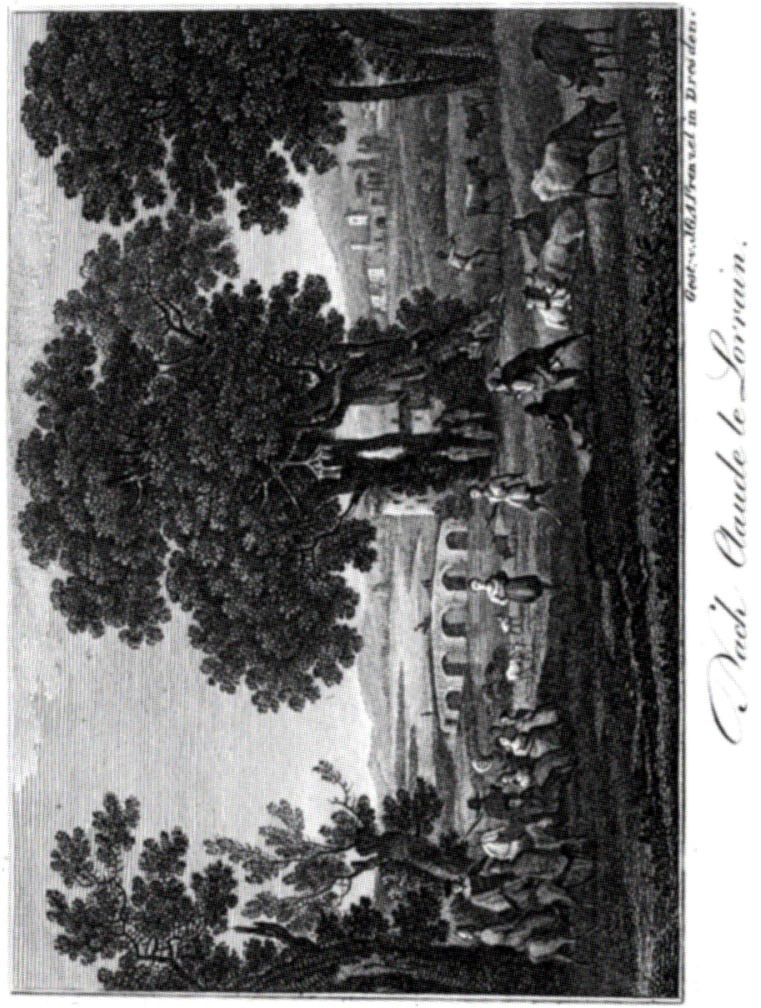





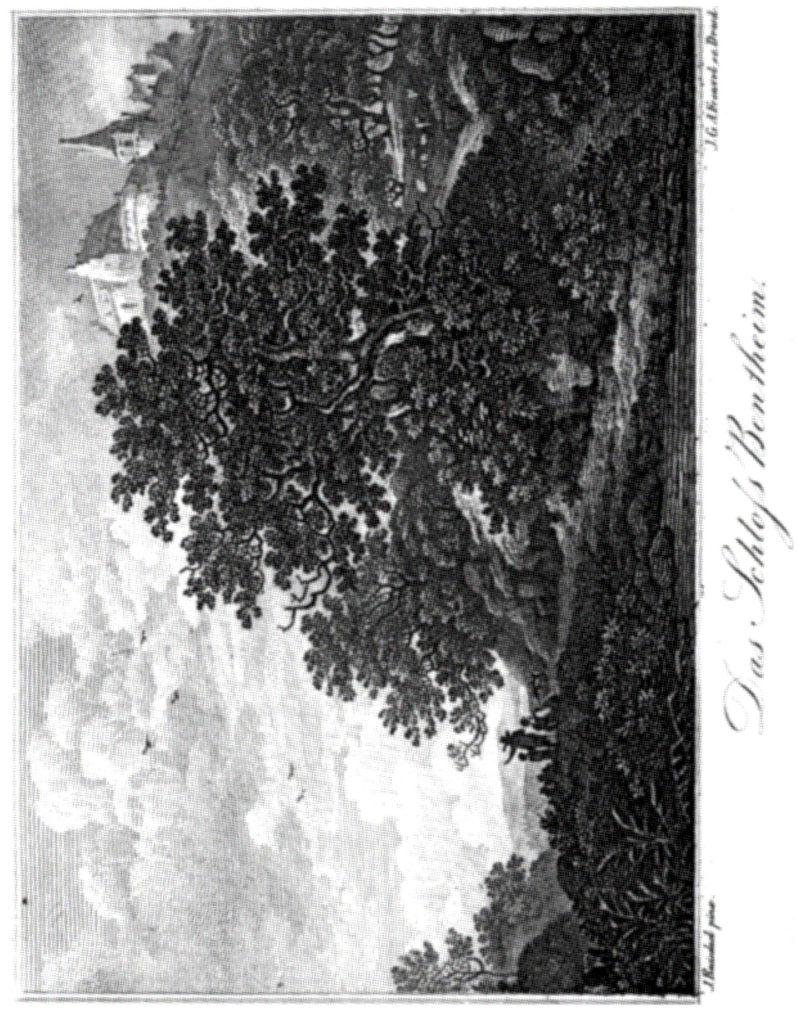





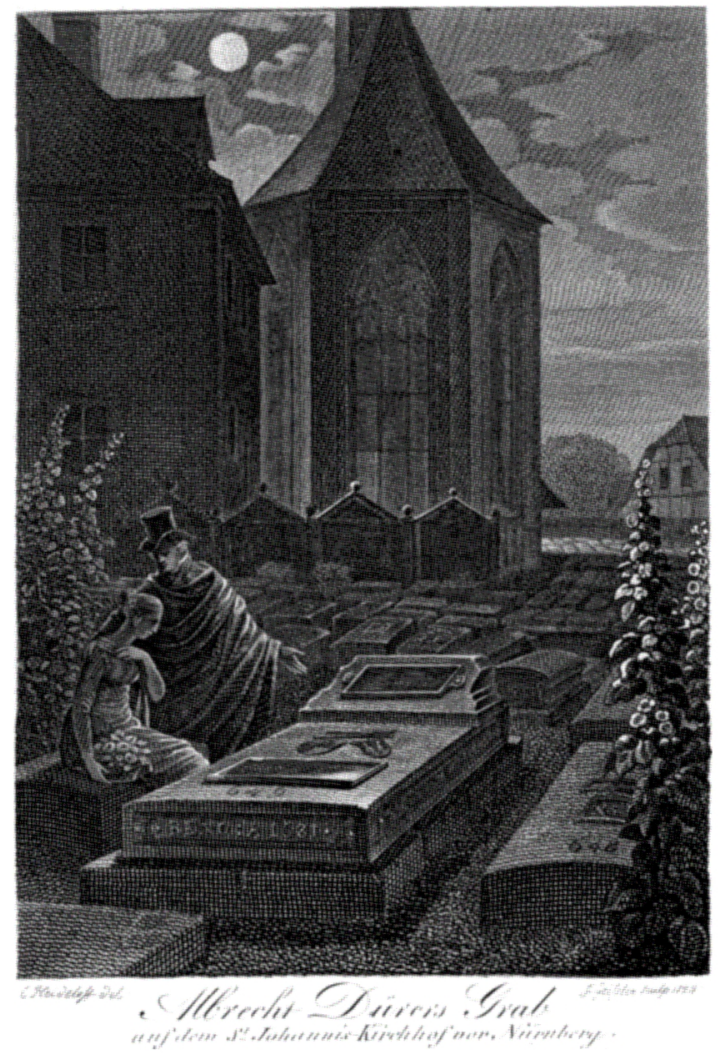





\section{Die $D$ ámoneninjel.}

I.

Jiron fam bom Spferfeft

Einer Injel bergezogen;

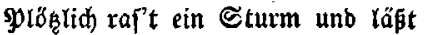

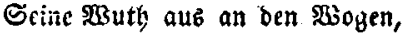

unb tein 2 neter bält megr feft.

als ifn fo von Stranb zu Stranb

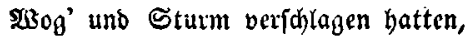
sahm - itsm gänzlich unbetannt Enolid) inn in fine Sohatten

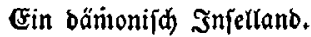

Für fein Sdjiff, bas unbaltbar In bem গampfe mit ben şellen uno bem Sturm gemorben war, Seut igm, um ez herzuftellen, Sutes \$olz bie Snjel bar. 
Boldh eint lieblidites (Semilath Feiner, wonnefúf:er Düft: 2uthmet jebez \&enzzebúj)! Zulez, şlumen, Ridyt unb Rúfte, Zules büntt inm znuberij̄.

Cine $\mathfrak{B z a r n u n g f t i m m e}$ fpridgt:

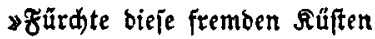
unb bieb jüße 3aubertidgt! Süngling, lap bid) nidt getưften! Irraue biefem Frieben nid)t!

Blinber Muth im fremben Raum Siann fid nur mit untheil lohnen;Dennod - angetanbet faum Juf ber Sñfel ber Dämonen Gällte Miton einen Baum.

Da verwanbelt fid) ber Fireio Seiner friebliden Befüble, Die er rol zu beuten weif; Selbft bie Ruft ber gaubentühle Brennt ihm Stirn und $23 a n g e n$ heif. 
Sđon verfünbet bie Sefage Sid) in fernen Donnertönen; Requvoll beut er, ben $\mathfrak{Z}$ tar Der Dämonen zu veriőhnen, Einen Baum ber ફ̧eimath bar.

Die Srafelftimme ipridgt:

\$ Sold ein Baum, ben Riebe weitte, Sey baz Spfer beiner pflidht! Bötterzonn ift bas Beleite Deß, Der ein Belübde bridt)!" Eüjen foll ex biefez Bano, (E) ex ruht bei feinen $\mathfrak{E a r e n}$ : unb nur fo läpt ign ber Strant Der Dämoneninjel fabren;

(Er etreid)t bas ફ̌eimathland.

Freubig fdlägt fein 5eerz. und faum Brebt ex auf befannten wregen Heber manden theuren Raum: Da tritt enda ihm entgegen, unter einem $\mathfrak{B}$ udjenbaum; 
Enda, wic ein Roicritamm ueppig błühend unb nicht minbes $\mathfrak{U}_{\mathfrak{r} g l o b}$ fróhlid), twie ein $\mathfrak{E a m m}$; Beibe Gatten fiton alb אinber Braut gepipielt uno $\mathfrak{B r a ́ u t i g a m . ~}$

Mit ihs wanbelt er fobann, Beibe wandeln froh zufammen, unter Fragen: 230 ? und rann? alinben fidh getheime frammen Still in beiber 5 serz̆ an.

Seitbem fand nun \&nda fein,

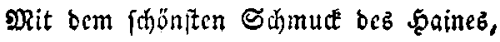
Morgens frïh vor Sonnenidein, Jmmer $f($ ) on betrïnzt ibr kleines, Grün umranttes Fenitertein.

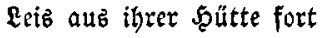

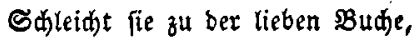
Sidit, ald ob fie an bem Drt Iigend etwas eben fudte; Dod) fie fanb ben \$aron bort. 
Brorbne 3eit ber liebe, bu

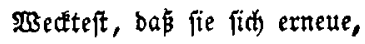
Deine $\mathfrak{W c l t}$ aus obcr $\Re u h$. $\mathfrak{D a}$, ba ¡dwur auch sieb unb Ireue Miron feiner $\mathfrak{E} \mathfrak{y} b \mathfrak{a}$ zu.

》) Miron" - rief fie, 》mein! idi bein! Whie cin Dopperlied burdbringet Midh bas Scil: mit bir zu fern! Mcine ganze Secle klinget Trie ein Ricberfeft im Şain - 》

Sitte marz auf Eyba'b Flur, \$3senn fich sicbezjinn vermählte, Das er fromm uno einfach nur Einen Baum zum Zempel wählte, \$Bo er feinen $\mathfrak{B u n d}$ befdrur.

und fie weigt ben füönen sraum, Den fie heilta pflegen wollte, şeibt ibn mit bem fidönften నraum, Den bie żeit erfüllen follte, Zeftlid cin zum Iempetraum. 
6

2ud) die $B$ ude wrigt fie ein!

auke Früglingžlerd)en fangen --

$\gg \mathfrak{R}^{\mathfrak{e} d}$ )

aber Miron fhaut befangen

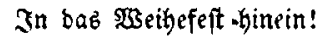

》Blicfe, Riebiter, blic cmpor!

Şöre diefe Brautgefänge! - 》

26ber feinem innern $\mathfrak{D h r}$

Shroben bie Sratelf́tänge

Der Dämonenintel vor.

2.

Sidht mebr blühten frifh uno thar \&nba's jugenblide : Trangen;

ueber ibren Frieben war

Eine böfe 马eit gegangen,

So ibr Samerz uno krib gebar. 
Ginjam rankt fie bin unb ber; unb beim Rlang ber $\mathfrak{E} e r d e n t i e b c r$, scsallt ibr suren bang und fdroer, Eeufzenb wallt er auf unb nieber, 》 Miron! Miron!" [eufzet cr.

$\gg$ Dort in jencm fitllen Saum, ISo cr mich zur Braut erforen, Steht niàt mebr ber heil'ge $2 B a u m$, Wo er Srette mix gefdworen; Rodh ift mir $c$ ह mie ein Iraum!

3euge war ber fänfte $\mathfrak{x} a g$, Der ba burch bie Sadbe glänzte; Rings bie frlur cin Feftgelag, Das ber Blumenmono befränzte,

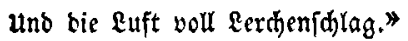
„ $D$ ba fdlug mein Serz fo froh, Wie ber Sdlag ber Lerhenlieber! अbr geliebten Eerben, 10 Sanget ibr nun nimmer wieber Seit mein epben mix entflob." 
》) IBahrer Rob ift folder (Gram;

rur bie Freub' ift wabre Reben, Die mit Miron zu mir Eam;

गुer Fann bie mir wiebergeben?

Der nur fann eb, ber fie nabm!*

\$) rie mar bie 3eit fo fdön, z's mid fcine lieb' entzüffte!

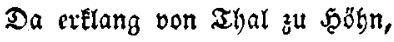
Die ber Frübling heller fdumülete, Riebefeliges Getön! 》

"Iret' id jegt in jenen Raum, 3u ber Mahlitatt fłöner Stunben : aules ftumm! ber Geilge Baum unires Sundes ift veridhwunden,

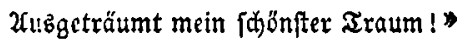

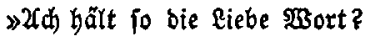
Nein id Eann - idh Eann's nidht faffen! Neine Budue muste bort Sid) zur Sarte madien laften, und bie trug ben fiebling fort." 
》) Aämpft ex mit bes Meeres ßBinden! 2Baz benn fud)t er fern von hier? Rohnt' or mehr alb Riebe finben? Eiebe fand ex ja bei mir! - 》

》) 2Arme Inba, ein Gelübb Sat ifn weg von bir gezogen! Fromme spflicht, getreu geübt, Nadht bie (3) btter hnz geroogen, Sb jie aud) bas Serz betrübt! - 》 》Şat ign eine fromme 9 flidit, Ein Belübs inn fortgetrieben? Fromme 9 flidht, bie ફ̧erzen bridht, Rönnen nidht bie (B)ötter lieben! Nein, bieß oürfen Götter nidht. -

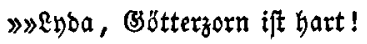
Enba! Enba! nidht vermeffen! - $\gg$ Zuer bie Berlapine ftarit, Wller (Sötter sRaht vergeffen, In bie tobe Begentwart, 
10

Troftlos irt fie an ben Strand, Iroftlos mantt fie auf unb nieber: \$ Meer, bu haft ifn mir entrandt! Wo cr weilet, gieb ihn wieber Nix zurüf, bu frember Lano!"

》) Fönnten bicfe 2(rme fidi) Einmal nur um ibn nod winben! Dod fein 2 uge würbe midh Nidjt mehr (d)ön und lieblid finden, Mteiner Xugen (3tanz exblich. "

$\gg$ Jebe $\Re$ of' ift vom Gefidt Zuf ber ahräne roeggefdwommen, Die aub meinem Şerzen bridt. Eerz und $\mathfrak{E}$ erditen find getommen, Zweer er - nein! ex lam nidbt. 》

》 Daz ift - o wie nenn' id's bod)? Jh⿱ bin ibm getreu geblieben! Selbft im Iobe werb' id nod Jhm verzeihen unb ihn lieben!

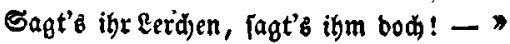


3.

Diron, fern im Sauscrbain

Einez frimben Injellanbeb,

Röjet frin Grchïbo, allcin

Sonder ringebent dсz Bandcz,

इae an syda mabnt, ju fign.

Da nun bat er opfind baar

abgethan, was פpflidit gefobert;

Die gerweibte $\$$ udje war

In ber Sdiffeform aufgelosert

$2 \mathrm{~m}$ båmoniןdhen 2atar.

3u bem lodénben Getön

Seines Dpferfeftes waren

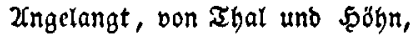
\$3oblgejdimüdte Nymphenjd)aaren,

alle ncugierreid uno fäön.

Jüngling, rüfte bich mit Muth!

Sich! es nabet beinen Rippen

Sid) ein Nel(t), voll 3̧aubergluth;

sibag' es nidt, baraus zu nippen!

Jüngling fen auf beiner șuth! 
2aber achtlos, wie er rat, Dreht er fidh im willen Reigen Giner bunten Nymphenistaar; Seine innern Stimmen fdweigen; Jhn umringet die (Sefahr.

Frei ift er, fein beilig ş3ort 3wingt ibn mebr, fich aufaubalten: Dennod rocilt er an bem Srt, $\mathfrak{z}_{0}$ bie finftern Mäd) walten, sosie gebunben fort und fort.

शBifl mit Inda'b Sulbgeftalt Die Erinnung ihn befdleiden: S bann mus die Fromme balb Einem wildern Taumel weid)en, Einer fünoliden Bewalt.

Smmerbar und immerbin Sălt ihn, wie an 3aubetbanbon, sweld)e mädtig feinen Sinn, Mäd)tig feinen (seift ummanben Murtilis bie 3auberin. 
(sinft an cinem muntarn $\mathfrak{B a d}$

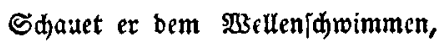
Dab gen $\mathfrak{B z e f t e n}$ eilte, nady; Sylöęlid werben Eerdenftimmen S̄n ben hoben lüften wad.

\$3eld ein $\Re$ uf! und ihn befällt 33 unberbar cin bumpfaz Sd)redten. "Ia, ihr 3ungen, fevb beftellt, Uนe bem Zauber mid) zu reden, Der midh hier gefangen hält! "

Tief bon inmerm ङdymerz befiegt, S3iro fein 2 uge trüb unb trüber, unb fein seerz im slide fliegt

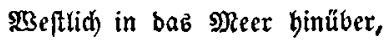
\$30 bie theure Sceimath liegt.

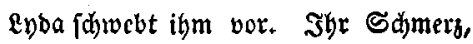
Seine ganze Seel' entführend,

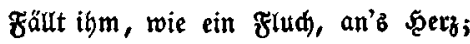
und ibr şlide, fo janft und rübrent, Bieght ihn mäd)tig beimatbmärtb. 
Sdion vorbeigezogen rar Mandez Sdiff, ex war geblieben Un bem Drte ber Befahr; Endidi) Eam ein Sddiff getrieben, Das ein Räuberfabrzeug war.

Und es naht und waht fid (d)on. " Männer," ruft er， 》läst eud rübren,

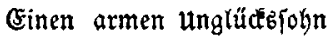
Seiner Sceimath zuzuführen! Id) veripred' eudt guten $\mathfrak{E o g n !}$ » 》 Sey bann, netmet ihr midh auf, (Sey) mit puch ber Bsötter Bnabe! und baz Gajiff lentet feinen Rauf Bum båmonifđen Beftabe, und ber $\Re$ äuber nimmt ign auf.

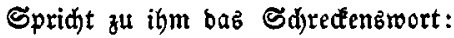

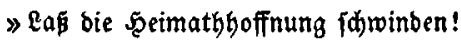
Sollft in einem guten port Jrgend eine Seeimath finden und fo flog bas fahrzeug fort. 
\SBeh!» ruft Miron, \$Boffroidt!

wer wirb einft an bir mich räthen!

(3ötter fínen zu (Serid)t,

Fitud) ereilet bas $\mathfrak{B e r b r c t h e n , ~}$

Straflos bleibt bie Sünbe niđht! -》

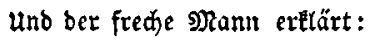

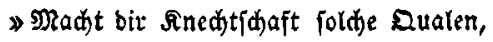
Run jo magit bu beinen \$rerth, Meiner Sđäg̨ung nẳ, bezahlen;

Mehr wirb nidgt oon bir kegehrt! -

⿰氵äährend ex jo böhn't und ladt, Biegt bas Sdjif mit guten \$Binben J6ent wirb's, ein Sturm errwaht; W3ic aus taufeno Feuerijhlünben Donnert bie Bewitternad)t.

Shrwarz verbůllt bie Sternemivelt, unb bas शreer, cin unerme Wreites Brab, bas fteigt uno fällt, Xugenbliaflid, aber gräplid Bon bem 
16

2rotll Szeaniben = \$Bzuth

Eine 2 zzelt barnieber würgen?

בaumelno raj't die \$3ogenfluth,

(S)eid) lebersigen (Sebiltrgen,

Shimmerns von ber Blize (Slut.

Sturmwind, ber von Sften pfin, Seulet pottend utz (Seminfal

Xuf bem Sdiff, unb jagt bas Sdiff,

Did)t an Mironb seimathinjel,

Sdmetterns auf ein Ferfentif.

und bie alte Sirippe Eradit,

Bon (Setrümmer rinģ umiăłvommen;

Siaum ijt, alb ber $\mathfrak{z a} \mathfrak{g}$ erroadht,

Raum cin Reben ifít entEommen

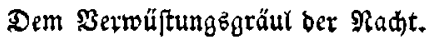


4.

eyba's Geele zagt und tlagt, Durrh bie graufen Nathtgeipenfter

Eirtes Traumz empor gejagt, (Eh) nod) um ihr șüttenfenfter \{eiz ein Morgenjthimmer tagt.

Tief in einem finftern $\Re$ aum Masar ihr Miron mit ben Mienen Gines Tobten, bap ex faum Siod 孔u Eennen war, exfdienen.

Sod) burdjidaubert fie ber Traum.

unb fie fpringt vom Eager auf, Wantt, im Innerften verniatet, 3̧u ber Bartenböh binauf, Sdjauet will umber und ridjtet Dann zum פiveer hin ihren Rauf.

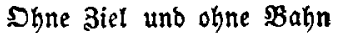
sosito fie fort unb fort getrieben; und in ihrem finftern $\mathfrak{\$ a} a \mathfrak{n}$ Siegt unb bört fie nidgt bie Rieben, w3eldhe fid ihr tröfteno nabn, 
Mit fdon barb erlofdnem Blid

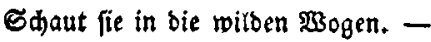
- Shr habt graujamlich baz (Slüdt Meines \&ebenz mir entzogen,

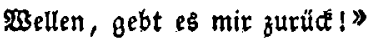

* Bebt ben holben Mann zurüd! Eapt mid ibn nod) einmal feben! Sollt id aud im 2ugenblice

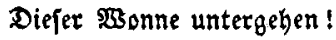
(S)önnet mir bas kurze BIüct! "

Starr bem Meere zugewanbt, Bebt fie ängittider bellommen. Sief)! was treibt bort an ben Strans Xd)! ein Eeidnam Eommt geidiwommen, unb ign wirft bie Fluth an's Ranb!

Didjt vor ihren Füpen ba Eegt bie Fluth ben Reidnam nieber. Dem Berfinken ijt fie nah, Wantend fliegt fie, naht fid wieber (3)ötter! waz erblictit fie ba! 


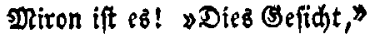

Sd)reit fie jammernb auf - wbie bleide

Sdrocigende Seftalt, fie fpridt:

» Miton bin idn! Mironz Reide -

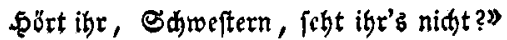

\Diefe Eippen, einft fo roth,

B3ie von Rojenblut umflofien,

Seht ! wie bleid), wie ftumm, wie tobt!

unb bas Zuge zugefdiloffen,

Dab fo holbe Grüpe bot!"

* W3ie nur! ad wie fann bas fern!

Deffne bid bu Strahlenauge!

Das id tief in mich binein

sidit auz bir unb geben fauge!

Deffne beinen Sonnenid)in!"

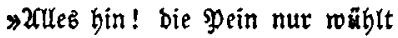

Jief in meincs \&ebens (Srunbe;

Waz mein armez

Fühlt jid an wie cine $\mathfrak{B}$ unbe,

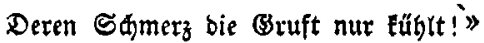


Futhtbar idhaut fie her unb bin, Furditbar ruft fie: 》Sdwarz berhangen Ift bie Robtenpflegerin!

Ift bie Sonne weggegangen?

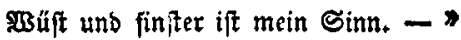

und fie fapt bie lalte Şanb, 2Birft (ith) auf bie Reidye nieber; Sthon bem \&eben halb entwanbt, Starren zudfenb ihre Brlieber, Bib fie plöglith fitc) ermannt.

und mit halb exftictem saut Ruft fie: - » Xuf zur 5ूodzeitfeier! פiron ift mir angetraut! Sebt mir meinen Spod)zeit = Sdleier!

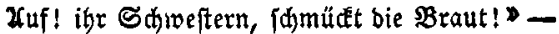

》) Rein! niđt auf! - nein etbentwätts! \$Beg! Ginweg mit freub' uno Ianze! flomm, bu füper, leçter Sd)merz! 2̧sindet RoEmarin zum Rranze spix unb ifm" - D̃a brach igr 5̧erz. - 
$230 h l$ mit mandhem Riebezppanb unb mit reidsen slumengaben, So bie Rieb' in Rränze roanb, 20 urben beibe binbegraben, 230 bie Gcilge Butbe fand.

Beildhen wirft ber Eenz bort $a b ;-$

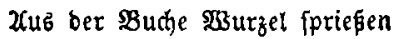
Surbe Reijer um bas (Gsab Beiber Riebenten uno biefen Eanfte Shatien brauf berab. B3enn ber leğte Strahl entfliebt, Noandelt bort ein teijez \$Beben, Daz burc) Laub uno Blumen ziebt, Sleid Der $\mathfrak{E}$ uft, in ber baz $\mathfrak{E}$ ben Cines Sharfentons veridicb. 
Der blinde Geigenfpieler. *)

Der Jahr'stag iff's, an bem suife, Die Sönigin, nach నampf unb Sieg, zum heimathlidien 9arabicje, ałz \$reupenz Sdutęgeift, aufwärtb ftieg; unaufgehaltne Iabränen floffen am Marmorbenemal ihrex Bruft, Das, jährlid) (Einmal bann exjdloffen, zur (Segenwart Bergangnes ruft.

Bergangnez, baz in (5röß' unt Mitbe vergeiftigt, fđöner nod) er[dicn! Drum nad Sharlottenburga Sefilbe fdeint beut gerwandert halb Bertin.

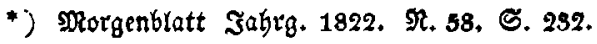


So pilgert Jungfrau, Marn uns Rnabe zu cinem frommen $\mathfrak{W}$ allfabrtzort, uno ferbit ber Greis am moridhen Stabe zidht babin hullfeabnenb fort.

3um Manbrungeziel, ber Brabeghate \&uifens, ftrömt bie Menโfbenflut, verionmolzen wie zum Sdnees Balle; in शु3irbel breht fid) Scut an \$̧ut, und während fidf bie bunte Nenge

balb wogent eint', balb töjend fajieb, fteht an ber Seite im Sebränge cin armer blinber Junualib.

Stod) fpriá, jelbit aus eritorbnem slicte ber शRut, ber bamals in ifm glomm, als er zuerift lie Shanazentüde trok ber Rattetidienfaat erflonm. Dort liep er, als hes Siegeb äeidan, ben red)ten fuf im 5opipital; erblinbet, mus er müisolf jhleidfen, entbebrent goloner Sonne Stral. 
24

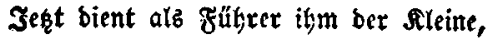
fein Sobn von fieben $\mathfrak{J a h r e n}$, und

fehlt biejer ifm, führt an ber \&eine

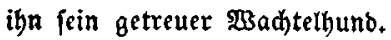

Dem Arrieger blieb, ftatt $\mathfrak{E}$ orbeerzweigen,

bie rothe Sharte auf ber Stirn,

unb - bie erbärmlidhte ber Beigen,

bejpannt mit fdled)tem Saitenzmirn.

Dex Beige war frit Sünglingetagen

ex mebr, alb ihm bie Ionkunft bold;

3eğt murte fargen Sinz fie tragen,

ergånzen feinen Bnabenjolb.

Bei frohem Janz, bei $\mathfrak{R a h l}$ unb Zeften

warb eingeftimmt unb aufgeppielt.

unb immer gab ex bac zum $\mathfrak{B e f t e n}$

wab אeiner für erträglid biett.

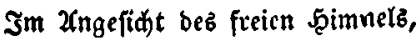

ber fegneno auf ben 2 trnen blict,

ftebt jeşt in Mitte bes (servimmels

oer Invalide, balb arbridt. 
Dod) hat bie Şänb' ex faum entzogen bem Drang', tritt bor er ftramm und barid, ftreidft mit אolfonitm ben $\mathfrak{B o g e n}$ uny fiebelt Znhalt $=$ Difiau's Maria).

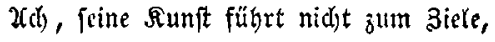
da fie ficin Mitleio ibm erflebt! Der Beifall feglt bem Minftrelfpiele, man bâlt bie $O \mathfrak{b r e n}$ zut uno geht!

Der אirieger fenkt bie thrönenfdroeren, gefololopnen Xugen ftill brab; wab fobern bern, bie mehr begehren? (5)s war fein 2đuleb, was er gab!

Da, wie zum Troft ein Gottgejandter, bringt auz bem bidten Menjonenjdwarm fum blinden Mann ein unbefannter und reipt bie Beig' ihm aub Die Zöne büpfen, toien, gleiten von bem fo falledt und mangelhaft bezognen Inftrument, fie ftreiten in şobllaut und in \$şed)[elfraft. 
Sunftfertig mebr, als jerbft vonnöthen, geráth bie : şeije zart und nett; bald ift es Ion von Şirtenflöten, Sboe balo, bals Flageolett.

Den Eauncngeift ber äne bänbigt ber Rünftler wie burd 3auberring, giebt, alb ber erfte Sag beenbigt, cin herrtides: God save the King!

Die Drpheuatŏne, rcin exgoffen, fie haben um bas Sunftgebiet ben bidten Sörertereis ge(d)loffen, ber na占 und nad fid weiter ziebt; benn fold) ein Spiel bon \$ghöbus Sobne auf offnem splas lodit nab und fern an Thüren, Fenfter, auf Baltone bie Damen und bie feinen Gerrn.

Sarofien balten auf bem glakge! Einftimmig ruft man: $\mathfrak{B} \circ$ u a $\mathfrak{r}^{*}$ ) fpielt!

-) Zllexanber von \$oudher, Ordefterbireftor Sr. 


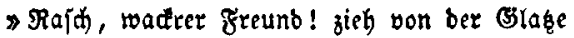

ben Şut!" - fo ruft er; Sener bielt

ben umgang an ber şand bes Sinaben

und Mitteio, Sunftinn, frober Muth,

fie warfen ibre reiden Gaben

bem Invaliben in ben Sุut.

Bei foldem unberbofften Segen

erglängt bez Iriegers Ingefidt.

Er ruft in biejem Silberregen:

》 Der alte Bott vertäst mid) nid)! -

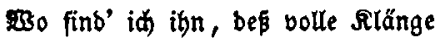

bas soantenbe Bertrau'n geftärtt? - -*

Dod biefer batte in ber Menge

(id) längft verloren unbemertt.

Arthur vom Rorofter.

Maj. Sart IV, yon Spanien; einer ber berühmtes ften Bioliniften. Er bielt fid lange in Jeutíntano auf. 
Sott helf in Deiner Roth.

sallade.

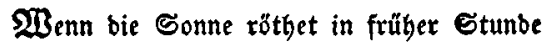

Die wcipen $\mathfrak{F} \mathfrak{c l j e n}$ am $\mathfrak{R c e r}$,

Shałken aub tiefem Meereb̉grunbe

(stodéentöne ber.

2(lo id), laufhend bem Beifterklange,

(Sing am Stranbe einft früh,

Sap eine Jungfrau am Steinabhange,

Sdöner fah id fie nie.

Die $\mathfrak{B}$ ellen fpülten vorüber am Steine,

2flz böten fie Morgengruf,

Jhre Blieber waren wie Sinnee fo rrite,

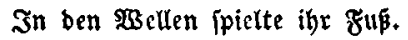

Sie ftrählte ibr Saar nadh vorn in Strägnen,

So gưldoen im Morgenjd)ein;

Sieber rollten ibre beisen aghränen

In ben Loden wie p̧ertenreign. 
Sie wu[d im weipen Meeresjhaume

$2 \mathrm{~cm}$ Steine ift feiben Beroand;

Эđ) aber ftand wie im tiefen Iraume

uno/riéb mein auge fo lang.

» Sd)öne Sungfrau bu, guten Morgen,

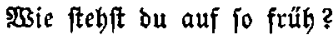

So früh in 2arbeit und in Sorgen

Sah ia cole Sungfraun nie."

Oa fdrą fie zujammen unb weinte

und falug bie $2 \mathrm{rm}$ ' in bie 5 höh:

s Shon wieber verloren!" - I็h meinte

Sie ptürzte fith in bie See.

- Sdöne Jungfrau, um Sottes \$rillen

(Sott helf in beiner Rotb!

Soll id beten für bid im Stillen

Sber lämpfen mit bem Iob?" -

Xuf fidlug fie thre gropen Xugen, unb farie: »3u fpät, zu ipät!

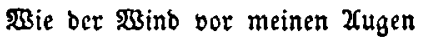

Jebe Şoffinung bod verwebt! - 


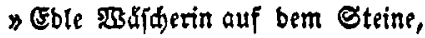

So fđön im Norgentoth,

ธag, warum must am Stranbe weinen

$\mathfrak{B} \circ \mathrm{bl}$ in fo tiefer Noth?》-

- Mein Bater, ber war ein großer אobnig,

Meine Mutter war fein Bemahl,

Stolge Füriten unb Spern unterthänig

Stanben in ifrem Saal.

Mit meinem $\mathfrak{B r u b e r}$ im $\mathfrak{\text { Rampf }}$ (i⿱千口) meffen

Modte fein Ritter gern.

Ji) felbft war eine bohe Srinzeffen,

Sebient von Rittern und Serrn.

Strablenb mit Bold = unb Silber = Sinnen,

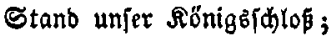

Seine నhû́rme lagen mitinnen

Ciner Stabt fo reid unb grop.

Mein Nater fikgt nun tief im meere

unb rauft ben weifen Bart,

$D$ baj meiner armen Mutter roăre

Gold bitter Roos geppart! 
grein lieber Bruber ift jeft ein Drade

und liegt auf rothem Bolb,

unb id, id weine, id weine unb rade

Db/nie bas stude wirb hols.

Die Stabt liegt veriunten im steeresgrunbe

NBobl joon an taupeno $\mathfrak{J a b r}$,

Seine Seele hat auf Erben Runbe,

Wie fie großs uno berrlih roar. -

- Sab̆ne Jungfrau, bu baft, wer bein Bruber,

Bater unb Nutter befannt,

Xber auper Bater und $\$$ Bruber

Nid)t mir ben Freier genannt.

Sie barg iffr Șaupt mit beiben 2Trmen,

unb fdhlub)zte wieber laut:

\Nenn Bott fid unjer wirb erccrmen,

ærert id meines Retters sraut.

Oft fah id früh bez Sonntags geben

am Stranb mand autagatinb,

Feiner bat midy weineno gefeben,

Sie gingen vorfiber gefdroint. 
Mand Sonntagkeinb [ah mid) liegen unb weinen, und ftürzte fpradiloz fort;

Du baft zu fpredien gewo $a_{\mathfrak{G}} \mathfrak{t}$ alleine

uno haft verfeblt baz $230 r t$.

D hätteft bu's gleid auşgefprod)en:

(Bott helf in beiner $\mathfrak{R}$ oth!

Dann wäre ber Zauber auf immer gebrodjen,

Эd) bein im Reben unb Iob.

D Menjabentinto, z̧u ipät verftanden Şaft bu ben redten Eaut.

Ed)loß und Stabt wäre auferftanben,

Id) beine reide $\mathfrak{B r a u t .}$

Nun werben bie (3)toden wicber Elingen

Bief unten aub bem Spe,

und alle meine Sungfraun fingen

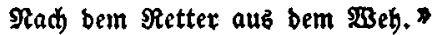

$\mathfrak{D a}$ \{hlugen an in ber Tiefe bie Slodten,

Die Jungfrau fprang vom Stein,

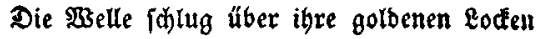

Sdäumeno fo weiß unb rein. 
Oft härt' idh Sonntage nod bie Broden

In früher Stunbe am $\mathfrak{R e e r}$

Aringen, ben fernen Retter zu locken,

Rie fah in bie sungfrau megr.

Billibalo Alexib.

Die Sagen von bem berfuntenen Binela, ben Blockentönen, welche ber Fif her in früther Sonntagss ftunbe bei heiterm fijönen Jungfralt, woldhe bem laufdenben Fifder einft zur jelben Stunbe erjojien, vercinigten fid) zu= fammen mit ben sitbern, welde bie grope Dftice bon bem majeftátijon Feljen Stubbentammer hers $a b$ gejehen, barbietet, roäbrend eines feçbroödentli= den Sommer $=$ Zufenthaltez auf bem romantifden Rügen zur vorftehenben, ben einfahen ßolfżton hof: fentlich nidft verleugnenden $B$ allabe. 


\section{Die Mofel $=\mathfrak{S} \mathfrak{d} \mathfrak{a}$. \\ Scenen a b ben tescten Jabren des oreipigjágrigen fiegb.}

》) Straflofe Fredhyeit fprid)t ben Sitten \$̧ohn und rohe Serben lagern fid, serwilbert Im langen firieg, auf bem verbeerten Soben."

Dieß jagt $\mathbb{S}$ diller yon ber 3eit, wo $\mathfrak{B}$ allen= fte in nod lebte. 3u weldher frutd $=$ und furdtbaren Uernote bie bamals erft geidopite Gaat päterbin ge= reift jen, werben bieje Blätte: - obroogl mit man=

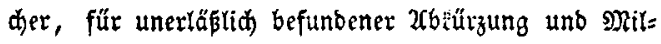
berung, bod im Şauptjäblichen treu nath ber $\mathfrak{B} e=$ fhreibung eines 2lugenzeugen - gnugiam beroäbren. Der Deutfije nimmt lebbaften 2 Untheil an ber $\mathfrak{B e r}=$ gangenheit frember Rationen; follte er an ben frü: 
Gern Drangfalen bez eigenen Baterlandz falt bor: übergeben? er ergöget fich an ben wunberbaren Sdjick= falen eines (s) if $\mathfrak{B} \mathfrak{I} \mathfrak{a} \mathfrak{b}$ unb ähnlidłer 2lbenteurer; follten bie 'Fährlicheiten eines deutichen Scodichülters nid)t auth anzichend füt inn feyn?

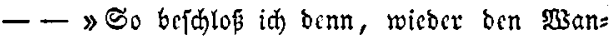
berftab zu exgreifen, ob fid vielleiăt anberwärtb ein

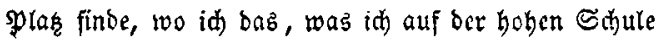
externt, in nüblidge 2rnwenount bringen fönne? Mein Reifegeräth war gut erharten, mein Stopdegen nod) ohne Roftlecte, mein Fchlijen bald gefdjürt, und, wiberrieth mir auch ber, in ben feibgurt eingcnähete 3ebrpfennig bie Eintebr in vornchme \$erbergen, to

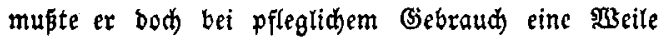
wiberkaltẹn.

Mit 2 (n)brude cinez frifden, jonnenhetlen Ingz ging id) burth bas spförttein bes Stäbthenz; bas gröpere ahor warb aus Beporgnißß vor Streifparthieen

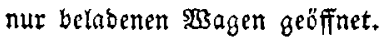

(Ein bejahrter Mann, vermuthlid) ber Mförtner, fás in einer Mauerblende, bie scände gefaltet, ein 
Itcines Buch auf bem Sdoofic; obne Brveifet wat ev mit bem Morgeniegen bejdäfitigt. $\mathfrak{U}(\vec{b}$ idi) ihm

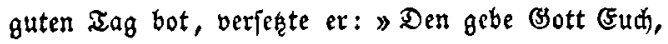

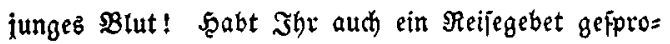
den? \$ָsarliă), Jhr bebürft nod mebr eines beglei= tenden Engets, als im alten Ieftamente bet junge Tobias!"

Sin ftugte zwar ein twenig, Iegte aber ful $B_{e}=$ jabung feiner Frage bie 5̧and aufs 5erz. Der B̧reib

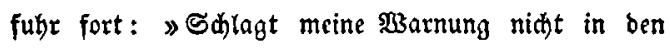
W3ino! Meibet vorzüglid bie şcerftrafe! ? Riemans ift in unfern בagen finer, felbit bie בobten in ber Erbe nicht! 》

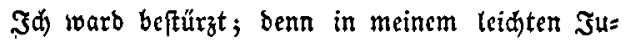
genbfinne hatte id) ber (Sefabren einer jekgigen $\Re$ Rije nid)t ernfflid gebacht. Dennod) idjämte id mid), als ein tüftiger, im $F e d$ ten verfudter (Sefell, אleinmuth zu verrathen, fohlug bie sinte anz Degengefäf, unb

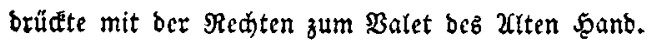

Raum war ith zwanzig Sdritte vom Ihore, alb

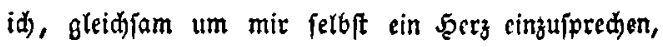


3u ben blauen Ruften Ginauf rief: »Cantabit vacuus coram latrone viator!"*) ober gu beutfd :

》 Sid)er unter Răubern fingt, \$3em im Sad fein SBąen fpringt! 》

》\$Bohlauf!" - fubr id rafder fortjdreitend in meinem Selbftgeipräc) fort - 》 Saăke trag' id

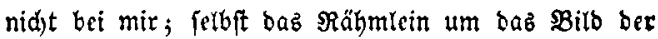

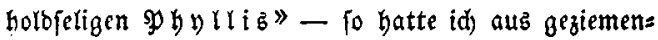
bex Beftheibrnbeit die liebrerthe Jungfraut Benannt, ber ervig mein Serz angebören wirb - 》felb/t bief Rähmlein ift bem Supfer nälyer, als scm Golbe vere want. Wohlauf benn! jebe Brille und Sorge weg= befungen vom 5eerzen!?

So hub ith benn mit beller Stimme, wetteiferno mit ben $\mathfrak{E e r d}$ en, bie aus ben braad liegenben Felbern aufflogen, cin Eieb an, bas idh fürglid in vertraus ter (Sejelfidaft bei Straf', anjonft in adj Tagen

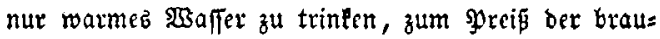
nen Mäbd)en, ober vielmebr meiner phollib, geoid): tet batte.

*) Juvonal. X. 22. 
38

(E⿱一) beginnt alfo :

3) Dunfeltraumes Eockentadr,

Duntelbraunes 2Uugenpaar,

Aönnen mix allin gefallen.

Braune Farb' eint feften Nuth,

3ărtrid) feit und füfre (Stut;

Braune Frudt ift füp oor allen!

\$Bräunliò ift bie reifite Traub'!

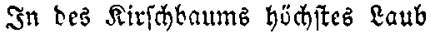

Steißt man nad) ben Slanz= Sorallen.

23az dic Sonn' mit Rieb' befdsint,

Wairb von brißsem Strahl gebräunt;

SBrauner Pfirficti lout vor allen!》

sch Gatte bieje Strophen völlig ungeftöt vor mid) hingejungen; benn auf ber ïben Lanoftrape grüfte nirgenbs ein \$3andrer, Eeinem burft' id banten. Die folgenden prießen nit guter Beleicnheit alle berühm= ten Brünetten, forvohl bez Simmps, alb ber Frbe, felbft bie f出warze, bor alfen Salomoniz nicht aubgeldhlofin. Sas Rieblein batte mir beim Rlange ber Relchgläfer red)t fein uno jier= 


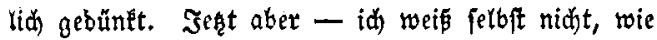
bas zuging - woltt' es mir wenig gnügen. 3 h) griff baher unter mein ledernez (soller, wo id ber geliebten 'Sungfrau Bill rooklverwahrt an ber $\mathfrak{B r u f t}$ trug, zog es herbor und reibete meine Blide baran.

Sdon Gatte id) bas (5onterfei breimal getüft und vertiefte mid, an einen Baum gelehnt, in zärtridhe Gebanten. Fben fiel eir Sonnenftrahl blizeno auf

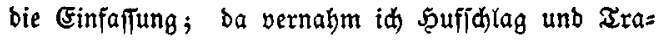
ben in ber Ferne. Sh) fah midi) allenthatben um. (Fine Sd)ar roftig und foübtg ausfebenber Reiter fprengte baher. Эd) verbarg augenblicklid mein Alleinob, und eilte, ba id mid bei weitem über= mannt fah, nah) einem, funfaig Sdhritte von ber Eanbftraße gelegenen alten Bsemäuer.

Nan batte minh nidt gewabrt. Die zerhuntpten Befellen ritten voruber, nad) ber Stabt zu. Ith blieb aub Beforgnifí, fie möbten zurübetegren, nod) eine Beile in ben verfallenen, ausgebrannten (Se= böft, unb batte inté żeit, bas ßBebenflidge ber an=

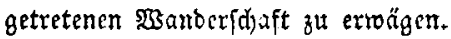

Ez war bamalz ein gar traurigez, fhredflides 
40

Reben; Entel unb Entelfinder werben bie Shilbee rung bavon für uebcrtreibung und Mäbrlein halten. Ränger, als ein Biertel = Säculum bindurh, batte

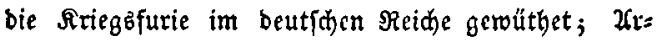

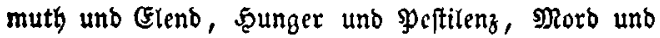
Branb bezeidneten allenthalben ibre Sdaritte. Eine

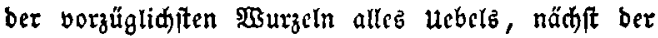
unbanfbarfeit ber Fürften und unerfättrid)en Şabgier ber Benerale, beftano barin, bofi ben Solbaten, burd) bie Betrügereten geiziger $\mathfrak{F e l b}=$ Sommiffarien, ber Sold vorentbalten warb. Dezhalb musten bie Rriegsoberften, wollten fie nicht geböhnt, beihimpft, ja unverfehenb arjdoffen werben, jebem unwejen ihrer untergebenen, obne nur bie $\mathfrak{A d}^{2}[\mathrm{eln}$ zu zuden, zuFehen. Soldyemnad) biente bie Sagung, meldie früberhin bon viel löblid)en Raijern ben Lanbblned): ten eingeidärft morben, nămliob), bā̄ ben Bauern, fo bas frelo beftellen unb bab ganze Menidhenge= fahlecht ernäbren, nidhts benn Şeu, Stroh, Sraut, \$ुsafter unb Solz genommen socrben folle, bloz zu einem Beipött. Die תriegbleute theilten fid nad eigner $\mathfrak{W}$ illeübr in Ratten, uno raubten, morbeten, 
verwǔfteten, verbrannten, was ihnen vortam. Dơ: fer unb Fleften, ganze Strafen ber Stäbte, ftanben verlafien. Die geflühteten Einwohner folgten, ans fänglid aus Sloth, nach Eurzer aeit aus Berwilde: rung, ben plünbernben 5̧orben. Şer ja norh ছ̧aus

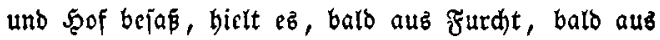
Bervinnjudt, mit ben herumftreifenden Soldaten. Die sauern berriethen ihre Ebelleute, bab Bsefinte

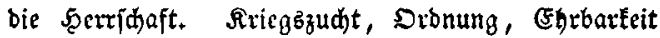
und Ireue, Sidherbeit bes Eigenthums, Grredtig= feit, Erbarmung unb Bottegfurdbt fahienen bänz̧lid yon bem blutgedüngten Eroboden veridgrunden.

$\mathfrak{U}$ tb id), was mir bievon im $\mathfrak{U}_{\text {gemeinen betannt }}$ war, in meinem Berfted bin unb her bedadft, ward mir ganz warm; iđh) wär gern it bas Stäbtchen, dab id) vor wenig Stunden vertaffen, zutüdigetebrt.

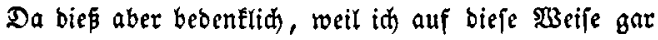
Ieidyt jenen Ranbreitern in bie şänbe laufen konnte, fo wählte id cinen jeitwärts führenden Fußiteig, uno getangte auf felkigem zu einer

Meine Şoffnung, bier roenigftens cine \$Bilohüter: ober నöblerhütte anzutreffen, blieb unerfüllt. Dod 
half mir ber mitgenommene Mundoorrath unb metne Reifcflajhe nothbürftig auz, und sies Mittagamahl, mobei ein Gtein ben Iijh, bas grüne Birlenlautb

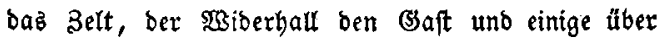
mir (d)Lagende Finfen bie Mujikanten abgaben, büntte

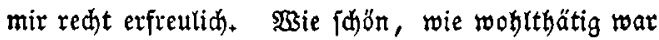
biefes milbe Gonnentidtt, biefe Ruhe, dicje tiefe Stille, zumal wenn id) baran badyte, waz brausen borgehe unb nun audh meiner wahricheinlich harre!

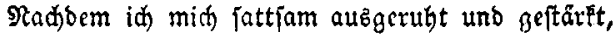
faste id) ben (Entidulus, 20les, was mir Bott auf= legen wolle, gebulbig uno männtich zu ertragen, unb überließ midy yon neuem ben einzigen şegroeifern, bie es hier gab, näm!ich ben übrrufeten, felten be= tretenen \$ुßstopfaden.

Uts idy bis zu Sonnenuntergang, balo burth

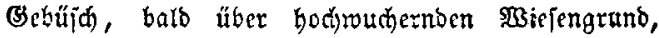
bald über unbebaute Felber gewanbert war, entbedte id in ber Ferne einen liften Sdyein und nahm meine

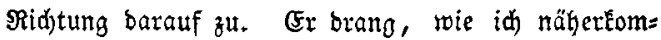
meno gewahr warb, aus ben, nur mit wenig Reften bon Błaz und Şlei verjehenen Bogenfenftern eines 


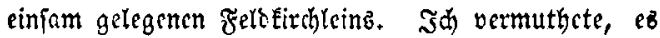
möbten Rrijenbe ober ctroa cinige Salzträger, ber= gleichen $\mathfrak{c}$ b viele in sortiger (J)egend gicbt, bajelbft ein unterlommen gerubt haben, in beren (Sefellidaft id) übernadten und am folgenden Morgen meine Reije fortiegen Eönne.

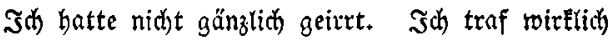
einige arme şanberer uno Salzträger, ingleiden zroei Sauflute aliz Düffelborf uno einen Boten, bot) aud lciber! viel 2andere.

Denn faum, baj it) dic Riuthpforte vorfidtig ge= offinet, un zu feken, wer fid bier bcfinbe, als midh pröglid) zuci aufipringende Männer binterrüçs an ben Xrmen ergriffon, mir thre piftolen auf bie $\mathfrak{B r u f t}$ fegten, Degen und Eeibguit nix abnatumen unb trostig befablen, midh fitll zu vertyalten, wolle id nid)t augenblidtich bes Iobre feyn! Seierauf ftieben fie midh in bie Nirche und warfen bie Iyür zu.

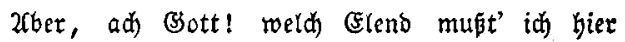
wahrnehmen. Neun gefattelte Sperbe, meift Sohim= mel, waren an baz \$̧ult eiltes langen $\mathfrak{B e t f t u b l}$ an= gebunocn und frafen aus פRaulfäcten; um ein gropes 


\section{4}

Feuer fapen eilf ftarke, furdhtbare, zum aheil fremb geťleibete Männer; bei cinem fleinern aber lagen ctride Feuerröhre und gegen zwanzig, mit Stridéen an einander gebunbene Bauern und 2(ndere. (5)enug, id warb alsbalb inne, baß id, um ben Eanbreitern zu entrinnen, aub bem Regen unter bie Traufe, nämlid unter eine 2lbtheilung ber Banbe geratben war, bie fith bamale, weit uno brcit gefürd)tet, zrifden Dem Rheine und ber Mojet herumtrieb unb beakglb bie Mojel = $\subseteq$ d) ar nannte.

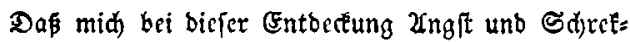

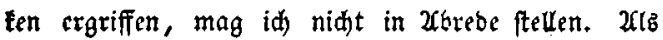
aber Cinige ber Berwaffneten aufiprangen uno midh Ieije fragten: wer idh jen? woher th fomme? galt ez Eein Räugnen. Donn Csincr von ihnen, Ramene SBattratoi Mutterpfennigen war, um fedzebn Dublonen mit ranzioniren Bebolfen, exkannte mich augenblictitit. Dies bradjte mir ben Bortheil, bas id) nidjt gleidh ben 2 ndern gebunben rarb, fonbern auf mein geges

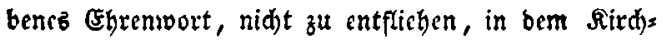
lein frei umbergeben burfte. $\Im a$, in furzem rief 
mid) SBattrami ans Feure, gab mir ein Stŭd SBrot

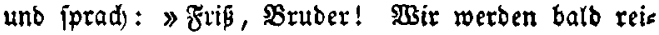
ten!" -

3roei Stunben vor Iagebanbrude ritten wir wire: (i⿱⿱亠䒑十) $a b$, nath bem (Scbirg zu.

Mid) batte Battramia binter (id) auf'z 9 ferb genommen; aber jämmerlich roar cz anzujeben, wie graujam bie anbern Befangenen zu fus fortgetrieben, unb, gleidh bem Sairh, mit speitjden = unb Säbel =

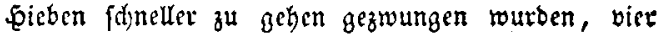
(\$ebundene immer zroijd)en vier wohlgerüfteten Infan= terijten und jedesmal 3mei zu Roffe zubinterbrein.

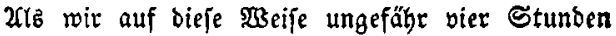
lang ber serghöhe zugetrabt waren, tamen woir in ein wilbcs, wüfes Thal. Şier wurbe zwijhen hohem

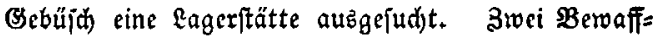
nete beftiegen bie hödfen Baummipfel, von wo aus fie die Strape uberjeken fonnten, unb wurben von zwei zu zivei Stunben abgelöft. Der ganze Irupp blieb an biejem Drte bis brei Stunben nah Dunfels merben. 
Die armen Befangenen erlitten inbes ben furdt: barften Scunger; Cttidhe berielben rupften Bras ab

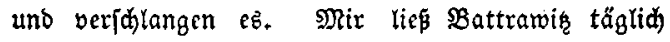
żwei Stüd Brot, orai 3rwiebeln uno ein wenig Salz reid)en. Şätte id bicinn, wie bie Folge lehren witb,

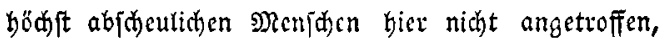
bätte ex ben Dienft, ben ith ihm geleiftet, nidjt gegen Feine Eameraben getübmt, böbłftwahriadeinlid wär'

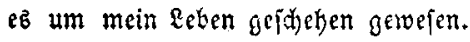

ZCK wir auf biefem Ylakge eine gute 3eit geraftet Gatten, zogen mich bie brei Bornebmften, nämlich ber Iange cseorg, ber für ben Sbriften galt, und Battrawi und Bobowi manbirten, auf bie Seite. Şeorg, ungefähr ein Dreipiger, war ein auzbünbig fäöner Mann, von

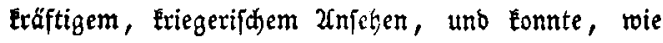
id) fpäterbin fattiam exfubr, unter biefẹn :Sebrroülfen immer nod) für ben zahmfren gelten. Die zwei Yekztern bingegen, beide Eroaten, gaken fowohl bem Zeupern, als bein Jnncm nad), bollsommen bas (segentyeil von ihm $\mathfrak{a b}$. 


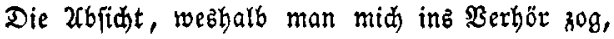
war teine anbere, alz fich zu erfundigen, wab id $g$ u $t$ willig für meine Xuslöjung geben wolle?

(od) ehe id Gicrüber 2 (ntwort ertheiten Eonnte, bie jeben Fadlz für bieje edien serren hödft unbefriedi= beno uno baher für mid) (ci)t betrübrid) aubgefallen jeyn würbe, gab eine ber Sdjitbrodjen ein 3eiden.

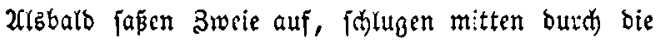

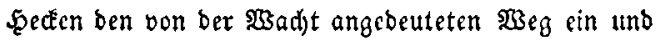
febrten in אurzem mit einem $\mathfrak{B a u e r b m a n n e ~ z u t u ̈ c , ~}$ ber zroijhen zwei Fingern ein idhmalez, eng bejdrie= benes 3ettelchen trug und es bem Dbriften überreid)te.

Da jebod biejer nur mühjerig, bie uebrigen aber bar nid)t lejen Eonnten, jo führten fie midh sines Stein= wurfe weit in's (Sefträuch und verlangten, id̆, als ein Belabrter, folle bab srieflein tefen, und da eb in franzblifider Sprade gefáricben war, verbolmetiden.

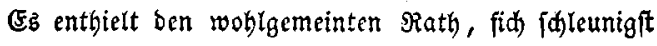
auf unb bavon zu madten; benn ber Gouverneur beb

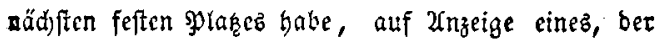

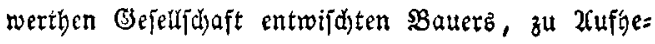
bung berfelben 2 nftalt getroffen. 
Die Znführer erwiejen fid jebr erzüunt, bas ber Brief nidht, wie fid's gebühre, abgefapt fey,

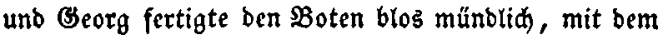
Befeble an ben $2 \mathfrak{b j e n b e r ~ a b , ~ k u ̈ n f t i g ~ a n b e r s ~ z u ~}$ fdureiben.

Mir aber batte meine Sprad)Eenntnis und Bereit= willigeit gropes 2fnjehen und 93 ohlwollen verjdafft. Man hielt mid) nun für bie (Sefellifhaft braud)bar unb ver(prad) mir, falls idt) nidtt lieber babei bleiben wolle, unentgeldidy fowohl bie Freiheit, ale fitd)reb Belcit bib zu jebem, mix bcliebigen Drte. Nur, fekte man bins

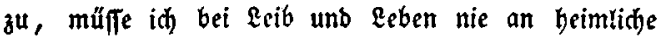
gludht benfen. - $\mathfrak{s} d$ fand, ba id jener aufage wes nig trauen fonnte, mid) zu ber $\mathfrak{X}$ cukerung berwogen, baß es mir zur zeit Gier $\mathfrak{x e d t}$ wo obl gefalle, unb gelobte 2lles, was man verlangte.

uebrigens jajen bie Furd)t vor ben, von bem Bou= verneux ergriffenen $\mathfrak{M a a b r e g e l n ~ k e i n e b i v e g z ~ f t a r k . ~}$ Szrenigftens nahm man nun ganz unbefangen einen nact) bem andern ber Mitgefangenen vor, und befragte

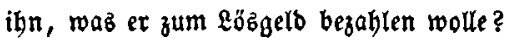


Der Xeltere von ben Düffelborfer Saufleuten ber: willigte alsbald hunbert Rei(f)ethaler, uns man war Damit zufriebcn. Der Jängere hingegen idhüg̨te vor, bie Stabt, beren Bürger er fey, lebe mit leiner ber kniegführenden Mähte in Feindidaft, folglid

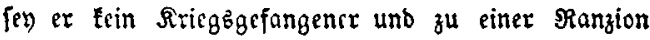
nid)t verbunden. Dodh ber arme Rarr muste jeine Wreizgcit finwex büben. Denn als man ihm mit bem ftarken Stichle eines Faufthammers Gunbert Streid) auf ben scib gegeben hatte, wäbrent 3̧wei ihn an ben 5eänden und 3wei an ben Füpen bielten, lernte er anbets über Neutralität urthcilen. Er veriprad ofne Borbringung fernerex Redteggrünoe hunbert unb

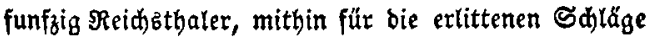
und auzgeftandenen Samerzen noch funfzig ayater mehr, ałb jein Elügerer Eollege.

Der aufgegriffene Bote madte einen $\mathfrak{B}^{\prime}$ rfuh,

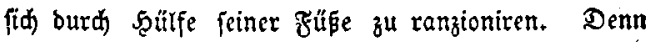
radbem ex feine Freilaffung um funfżig Reid)etbaler behanbelt, man iín lozgebunden und einftroilen zu W3artung ber Mferce beauftragt batte, erfah er fid bie (Setegentheit, bavon zu fhleiden umb verbarg fich in 
50

einen Şajelbuja). Nur bemerte man leiber bies bar balb unb Dreie zu פpferbe umfreiften ibu. Xub Berzoeiflung fprong er bis an ben Şalb in einen Teid), und ftrengte (id) an, binburd) zu waten; allein,

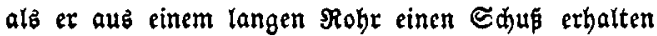
batte, unb nun, rogen (tebor nod) unerzogener אin: ber, flebentlid um Erbarmen bat, fagte ifm zlwar Einer bas eeben zu, ein Unberer aber jpaltete ifm, jobalb ex heraubgefommen, mit bem Säbel ben אopf

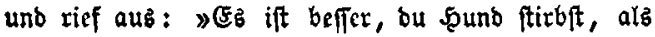
baß wir 2ulle verratben werden; $3 \mathfrak{s} r$ Zndern nebmt an ibm ein Beifpiel! feinem, ber Bleides verjudht, foll es beffer ergeben!"

Bon ben ubrigen Befangenen mupte ein Sdult= theip bunbert Reidsthaler uno ein Pfero beripreden. Die meiften aber entrdulbigten fich mit unmöglic) feit unb Xmmuth, wie benn in ber $\mathfrak{x}$ hat brei von ihnen, jebr rüftige SBauerpurjhe, wegen Mangels an un: terfommen, lange 3eit ihr sBrot vor ben Shüren ge= fucht batten.

SWeil nun Reiner mebr etwaz verwilligen toollte

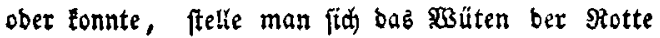


und bie Slualen vor, weldhe ben unglürtiden zuge: fügt wurben.

Meine freber hat mir johon vorbin faft ben Dienit verfagt, unb nux bie 25bficht, ben Eejer auf ben rid)= tigen Gefiahtepunct zu ftellen uno inn aus Einigem auf bas uebrige fhlteken zu laffen, hat mir nidt gänzlid)es Beridineigen geftattet. Xber bieß jdauber= volle sBilo weiter aufzurolleu, bin id nidyt vermö= genb. Sur foviel werbe hier nod) bemert, baß zwei Bauerfnechte, bie allererft, wie man bas nannte, unter (Sewcbr getreten waren, ihre vorigen Brother: ren aus $\mathfrak{B o b h e i t , ~ o b e r , ~ w i e ~ f i e ~ b e h a u p t e t e n , ~ u m ~ f i d ~}$ für früher erfabrne ungcred)tigkeiten uno Mips hanblungen zu räden, unter ben fredften Reben, weshalb fie bon ben säubern getobt wurben, auf fo

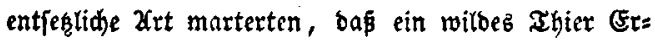
barmen gefüglt haben würbe. Einer biefer făänoli= hen Buben ift, wie id fpäterbin erfahren, crtappt, unb wegen anberer unthaten geviertheilt worben.

Fh) Fonnte midh bei biefen Graujanteiten faum bes Weinens erwebren; ich bat \$attramis, ben id jo für meinen Bönner anfehen mupte, auf sas beweg= 
lidjte, an Gott unb jein Shewiifen zu benfen unb ber

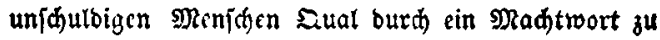
enben. Dod ber rief mix mit grimmigem Blic zu:

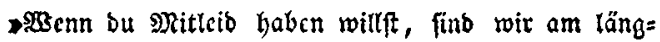
ften Freunbe gerwejen. Deb Teufets ift, wer von Nitteib roeī ! ”

23äbrend fid bieß ereignet hatte, modte, foviel id) nad) tem Raufe ber Sonne fhlof, bie britte Radmittagbftumbe berangetommen fern. $\mathfrak{D a}$ rief abermalz ciner ter ausgeftellten soften vom Baume, ex febe einen Mann; ber allem Xnjaheine nad etr wünjibte Sotjd)aft bringe.

So verbielt $\mathfrak{e z}$ (fid) audh, wenigftens nid) ber Znjidi)t biefer ftraßerfegerijhen Befellen. Denn ber Eerannahente war ein Sdjurfe, (nur mod)t's ibm ber Beier jegt in's (Beficht [agen!) war ein אunbs=

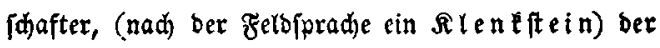

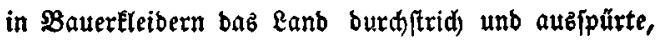
wo eine gute $\mathfrak{B e u t e}$ zu mad,en (ey).

Sobalb man inn capannt batte, zog er aub bem Ohx ein, in eine אugel zufantmengetellteb Brieflein, bas i申 abermale ben Xnfühtern lefen muste. Diejes 
nun war in ber fetbipradje abgefapt, bie idi) bamals frcylid) nod) nicht verftand, bod in ber Forge, wähs rent meineb unfrcirilligen 2ufenthaltez bei biefer

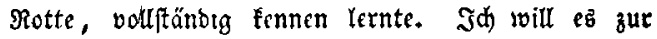

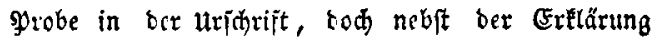
hicr mittheilen:

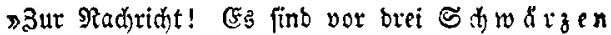

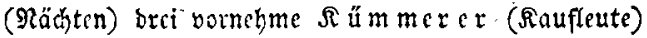

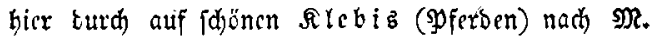
cafalt (geritten). Die werben nad) bret Sdyăr: zen rieber zurüle (d) wenzen (Befen) unb ctlide (S I eiden (Rcifegejullidafter) mit viclen baaren Me $\int\{\mathfrak{e} n$ (bielem baaren (Belte) mitbringen. Sie bas

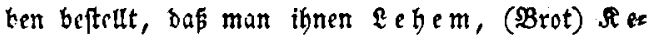
riß, (⿱⺈

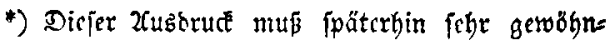
lid) worben fern; ;enn ex fintet fid einige Mal, wies wohl zufammengrzugen, $($ Füntel $=$ Fod) therz Gebidten. Uebcrbaupt bürfte tab bamalige

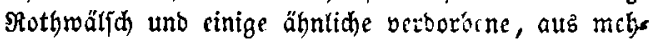

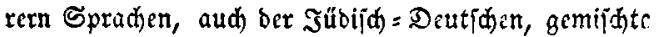
Dialecte von ben Spradforid)ern nicít genglid jut 
54

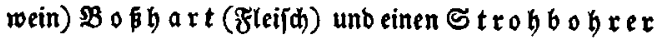
(cine Sanb) zu $\mathfrak{R}$. briffen (auftragen) foll. Denn fie wollen bajelbft fdobern (effen unb trinken.) Der $\mathcal{S}$ 中ö unb fie fo lange menteln, (aufbalten) bas ibr fie im $\mathfrak{S}$ d) of erbeth (F̧irthabaub) ober bod) im (S f ar (Dorfe) auf bem Da a

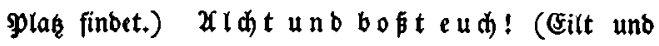
fdiweigt!) (Sute Sdivărz! ”

Rad) Berlejung biejes \$Briefleins warb ben \$fer: ben albbald Futter vorgeworfen, unb nath Berflus einer Stunbe faßen wir auf. $\mathfrak{J} d$ ) warb wieber mit auf ein Pferb genommen; bie übrigen Brfangenen

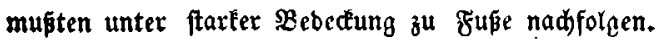

Bir ritten obne anzubalten $\{$ ed)s Stunben. Daun tehrten wir in einem alten, auggebrannten Sdloffe ein, baz berwiß feit vielen Jabren bloß ben Gulen unb Flebermäuren zur $\$ 30 h n u n g$ gebient batte. No(b)

vernad̆läpigen fenn. Ste ertlären Mandes in (Shro= nilen uno anbern wertboollen Sdriften, wab an= fonft mit ber Beit völig unverftänblid roiro. 
vor Berflú einer Stunbe fam ein, nath bem Sdnitt ber Şaare zu urthcilen, vorbem Solbat Berefener Bauer, unb bradte etlid)e Brote neb/t einem Fäplein F3ein; bent bie 5erren Straßenfeger hatten allent= balben ihre Bertrauten unb 3uträger, auf weldbe fie fih), Fomohl wegen (Sleid)beit ber (Sefinnungen, als

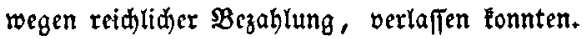

झुsir apen uno tranfen bei einem feuer unter cinem niebrigen Shuppen, unb nadbem ber Martes tenber mit einem Trinfgelbe von zroet Dutaten ent= Iaffen worben war, zogen wir twieber bei blinfendem

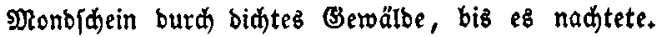
Man warb in bet Entfernung ein \$irthbhaus geroahr. Einer ritt bib auf einen Büdjenfdus barauf $z$ u, ftieg $a b$, fdnallte bie Sposen $a b$ uno falid zu Fuf

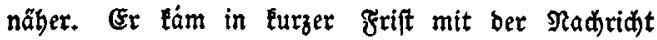
zurï̌, baß er hinterm Dorfe mit bem swirthe ge= iproden unb von ifm erfabren habe, es feb seit; benn die Reifenben wären in igrer Stube uno fdliefen.

2ugenblicklich ritten wir alle fort, fort, fort, unb famen, wie mir im Finftern büntte, an eine binter: 
56

thür. Die Reiter 備en $a b$; id unb nod ciner mus= ten bie Yyferbe halten; jene aber gingen burd) bie

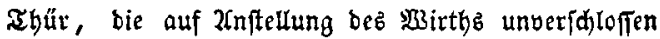
geblieben war, mit aufgerogenen siftolen ins saub.

(E) gefdah ein cinziger હdytis in bie Stube, aber biefer war aud̆ hinreichend, bie fỉhern Shläfer auffujd)reden und in Tobçangit zu veriegen. (Es wurben threr Fünfe gefangen genommen; cin $\left.\mho_{e} d\right)=$ fter hatte nod zeitig genug Eunte gerodien und wa: Durd) cin Fenfter entiprungen.

Die Befungenen nurben fämmtlid), faft obne cin खort zu vertieren, getnebelt uno nebft ihren idwer ren Felleijon fortgefübrt. Eictori muste id) mid) über

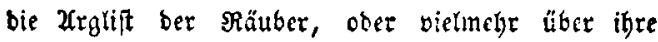
Beübthcit in Sdetmftrciden verwunbern, ja, bic 20sabrheit au befennen, fo tiefiz Mitleio id) mit ben unglüctiden fühlte und fo tüfter meine ganze (bes mütheftimmung war, in fonnte mid) einez frampfar tigen Radens nidt erwebren. Denn natistem bie Sdmapphänne bie Saufleute zu zwet und brei mit

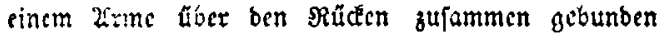
Fatten, nargmin fie ignen ben Neftet bes Baiutleibs, 
fo bas biefe armen, zum Theil betngten Mănner eb ftets mit bor nod) freicn 5̧ano balten mußsten, folgs lid) an ein Entraufon ober Bertitiden nidht benten tonnten.

Woir gelangtrit gegen Morgen in bie vorige Raubs boble, wo fid berrits tor geftern ia gerofine sBauex acbft nod cincm anocrn, beibe șsern, Brot uno Fleijh) in ueberf(us Geritiaffend, wieber eingefunten yatte. Wud waren intef einige Undere von ber (S) follidjaft, aud mit einizen Befangenen, hier einger troffen, und $p$ ga gab gegenjeitig einen gar lebhaften, jubelvollen Empfang. Mid fek̨te, beiläufib Befagt,

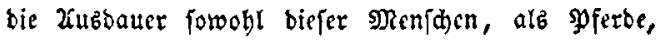
Göd)lich) in (Erftaunen. Joh war fo ermabet, baß id mid) fehr nad) cincr Streu fignte; abcr fie fömmtlith Gatten nod) fo frifde Jugen wie Falten.

Sunmehr ging eह gar luftig uno hor ż山. 2(ud) id) Surfte midy bem nidit entzichen, fah es gleidit in meinem Bemüthe ganz anders aus, fowoht wegen ser (Seroalttyaten unb (sraufamesiten, wowon id Beuge fert muste, als weil id furdste, es mödte einmal beifen: Matgefangen, mitgebangen! Die eb= 
58

Ien şerren bingegen hielten fith, ba biejer Streit) fo wohl gelungen war, für überzeugt, bas id von nun an mit ihnen gleides Ried fingen werbe. Sie ertlärten mir baher ohne umidweife, baß id berwehrt unb beritten gemadt werben, audh an ber Eünftigen Beute einen 2 ntheil empfangen folle. it) geftalten Sacten nad) bieß bobe (Slüd ausjdjlagen? Nan begann nun, bie gemadte Beute zufammen zu bringen und aufzubäufen. Es fanb fid an B̧etb

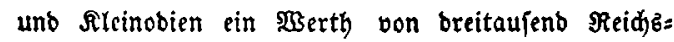
thalern, unb bas baare Belb warb in brei Theile ges theilt. Einen berielben betamen bie Musquetiere, weld)e bie frübcrn Bcfangenen nad)gebradht batten unb jeşt im গ̧zarbe bewahten; ber zroeite rourbe mit guter $\mathfrak{B o r j i d}$ t auf ben Rothfall zurückelegt, falls einer verwunbet würbe ober ein \$ferb zu Sdan= ben gieng, und id mufte benfelben, fey ez nun, baß man mid) für ben ehrlid)fen, ober für ben nod) furd)t= famften in Stehlen bielt, in Berwabrung negmen; ben britten theilten fie unter fid, , fo daj auf Seben

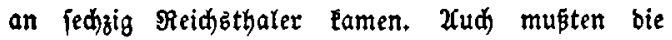

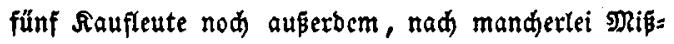




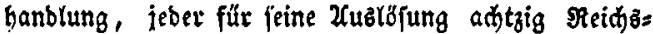
thater verwilligen.

unter ben Befangenen, welde von ben neu anges Eommenen (s)enofien mitgebracht worben waren, be= fand fid auth ein Doctor ber $\mathfrak{X}_{\mathfrak{z}}$ neifunft, ber fid ben Ramen $\mathfrak{s}$ a ncrati $\mathfrak{u}$ b gab. Sie in meinem $\mathfrak{l}_{e}=$ ben habe idy einen Meniden gefehen, in bem fith fo

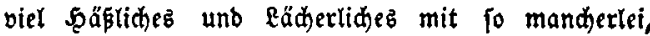

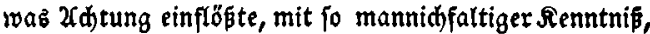
mit fo groper Berwandtheit, 2Anbere zu burdjdatuen unb igre Sdjwadjheiten zu benuzzen, vereinigt bătte.

Er war von Geftalt faft zroergartig, babei auf ber redften Sdufter mit einem 5eöderer verfehen, unb batte fo bünne Spindelfüpe, fo lange Enöderne Fin=

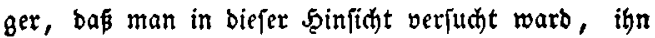
für cin Skelet zu halten. Seine ફ̧autfarbe bagegen

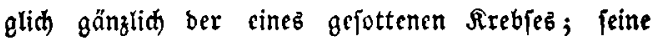
Stirn war faft vicrectigt, jein \$̧aar aus \$Beiß unb Sdroarz geulijht unb fehr fruppig; unter ben bu= fohigen 2 Lugbraunen funkelten Ereuzroeis gelbe, faben= artige Jugen fdarf unb feurig herbor, und ben Flei= nen, eingefniffenen Mund überidattete eine geroaltige 
60

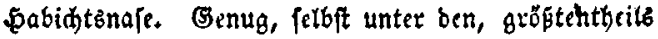

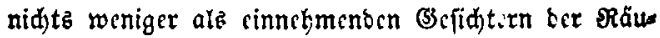
ber, glid) baz feinige, abjijreceno unb zum eaden

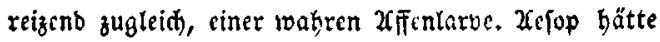
neben inm nod eine redit leiblitie Figur geppiclt.

Wie ber Şerr Doctor in \$Bahrbeit beife, und was für ein Ranbsmann $\mathfrak{a r}$ jey) ?habe id exft reit fpäter crfabren. Er fprad, obwohl mit einem fone berbaren, fdjarfon, faft fingenben Zccent, alle älteve unb neucre Spraden; mir wollte $c$ mandmal

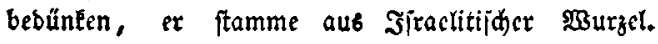
Daß $\mathfrak{c}$ in ber Sceilfunft crfabren fey, envies fid in ber Folge bei borfallenoen Bleffuren, io ber Mene iden, als פpferbe. Waser aud in allen üsrigen $\mathfrak{B i f f e n}$ idaften, felbft Eabbala, K(ftrologic und W(d)ymie

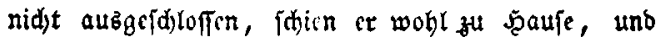

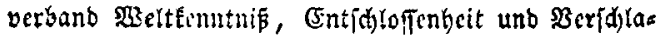
gengeit mit böd)ft feltjamer, felbif in ber grdisten (5) efabr ihn nidht vertaffenber $\mathfrak{E} a$ une.

Sein auffarlenber Xeuferez, fo wie fin ftete un ruhigeb Benebmen, anegte gleid hrim exiten $\mathfrak{X n}_{\text {s }}$ blidie, nämlich als ex nebft einigen 2 (ndbern vor ben 
Soriften gefanti ward, meine Xufmertjamerit. Ex benatm (h) bri bim gerochnlidem Berbär eben po vorfidtig, als feft; mir fam es vor, alb brüclee ce bem Beorg/etroa unvermerft in bie J̧and. W3ie bem jern modte, genug - er roar ber Eingige, ber fein \&öfegctb fu vervilligen brautte, wogl aber ves= fpreden muste, bei ber Bejellidaft zu bleiben uno iñ Dienfte zu leiften.

Sobalb er firi) auf bieje \$reije zum Staabs = Xrat ber lüblichen Mojeliđaar hatte anwerben laften, fteuten uns Bscorg und Battrarvis, id glaube, um fiti) einen Spā̄ zu madjen, alz Getahrte einanber vor, und sb id wohl bes 5̧errn Pancratius Doctor= but gebübrenb ebrte, trug id bod), zumal ba ex gar febe einem (şarlatan ähnlid) fah, tein $\mathfrak{B e b e n k e n , ~ i h m ~ m i t ~}$ einem Şanbrerébgruße, nämlid mit bemt: »Solamen miserûm socios habuisse malorum !” aufben $3 \mathfrak{a h n}$ zu füglen. Er ermiberte aldbald aufs fertigfte: 》Infandum jubes, domine confrater, reaovare dolorem!*)

*) \$Bedjelmorte Det Dibo uno bes Ireneas beim sirgil, bier zum İheil traveftirt. 
6.2

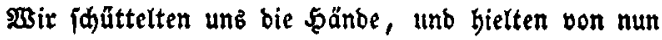
an, wo eb (idi) fügte, zufammen.

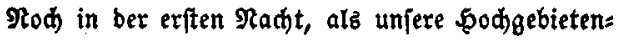
ben auf bas unmäprgfte zed)ten unb lärmten, folglich an Sdlaf nidjt zu benken war, ließ id bem neucn

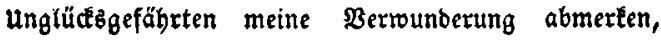
wie aud) ex, beffen Beridhlagenheit mir immer ein= leud)tenber werbe, inz Barn gefallen fen?

\ฐ̃a, ja Süben, bie Rajenipizze nad) Rorben jajiebenb $\rightarrow$ wer fterblide Menidh tönnte oft ziemlid geideibt fenn,

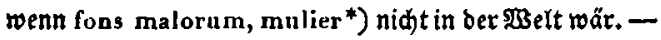

SRine Reugier warb inmer reger. Ith bat ith um nähere (čläuterung, unb er ertlärte fid), unter

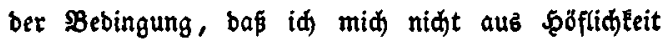
etra verftelle, fonbern ihm nad selieben gerabez= wegs ins (Befid)t lade, für bazu bereitwillig.

》Nod) vor ungefähr zwei \$roden" - begann ex, inbem er feinem (Seierijhnabel mit ber Spiz̧e bes 3eigefingers eine (5bre erroies - „befand id mid nebft

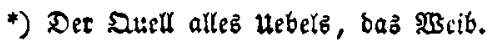


einer Renge ftattlider Reijegefäbrten uno Diener id) felbft, wie fie zalle, wohl belwaffnet, einen tüdtis gen Degen umgeidnallt, jharfgelabene piftolen in ben Solftern, unter mir einen präd)tigen, glänzend=

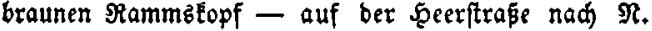
Me hercle! baż wax ein \$fferb(h)n, Baccalauree! iđd faß barauf wie Zlleranber auf bem Bucephalub, wie Perjeub, als er bie Xnbrometa rettete! Jh) war jeboch nux im \$egriff, zu bem beutiden friegsoolke zu ziehen, wohin mid ein vormehmer Patient be: juieben.

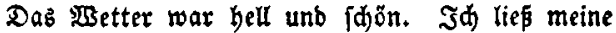
Xugen überall berumidweifen, unb - arrige aures, Pamphile !*-) gewabrte in einem, etroa zroeithun= bert Sdritte yon ber Landfrape gelegenen Gebülde sine gar feine, fdlanke weiblide (S) ftalt.

Mcine \&uft, mix bie ङdjöne näger zut bejehen, mad̆te mid) hödjt unruhig; bor Sattel unter mir

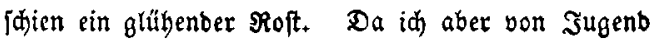
auf geroobnt bin, in Bejellijhaft anderer Männer von

*) SFig' bie Dhren, Şamphitus! 
64

bem frouenoolte immer zuletet beäugelt zu werben, bie $\beta$ aud Eürzlid in einer 5 erberge unter meinen ber: maligen Reifegefellen aufe neite erfagren hatte, fo bebielt ith, um cinmal alletn 5ankn im Rorbe zu jenn, meine anzenebme Entbeckung veizlid für mich. ”

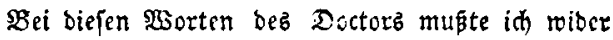
Millen von ber extheilten Ertaubníp (Sicbrauch mas

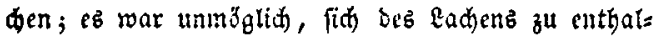
ten. Scr Doctor, ber eben trant, nur bon cinigen büftern :Banblidtern befolienen, glid in feinem Spiz̨entragen, mit bem gelben $\mathfrak{B}^{2}$ ämblein. unb tothen Doctormäntlein, mit ben fahlen, som Regen uno grzinb gebleid)ten unb zerrupften Sutfebern, mit fei= nen Groken Stulpitiefeln uno Sporen, in That uns झुahrheit einem Saushabne.

Sebr gelaffen fubr er fort: 》Э̧ blieb bezhalb binter meinen Peijegefábrten zurŭç unb beugte exit, ba jene wegen ciner (Ede tes :segz mid) nid)t mehr

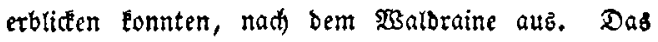

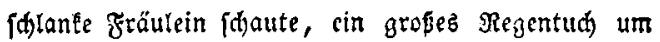
ben Ropf geidlagen, tiefïnnig vor fich nieber uno fithien midh anfänglid nidst zu bemerEen. $D \mathfrak{a}$ id 


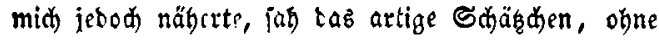
cben zu crid)redfer, auf, unb errviberte meine 2rntebe befd)riben und beffich.

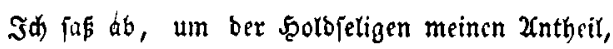

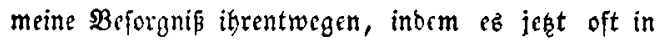

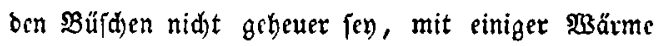
anz 5erz zul Iegen. Joh fragte nad) ihren Reifebe= glcitern. Sie blicte mid cine \$scike redt wumber= bar an, lähthelte bann ein menig - ungemein reizenb unb crbob aufe neuc mit cinem tiefen Seufzer bie lefir auebrutasvollen 2rugen.

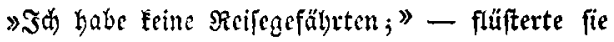

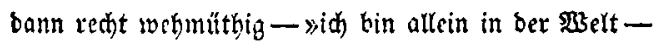
pine arme $\mathfrak{B s a i j e , ~ o b w o h l ~ v o n ~ g u t e m ~ S t a n t e ! ~ M e i n e ~}$ Uettern wurben bei einem näd)tiden ueberfalle un= feres Gdjlofies getöotet; idh ferbft entging nur burd) ein Wunder ben fhon um mid lobernben Flammen. Jek̨t bin id) auf Der $\Re$ cife, um bei Znnermandten, bie fïh in $\Re$, auffalten fullen, Sdugh für meine unerfabrne sugend zu fudjon. Fine bornckme Dame, bie im Yeseten Saditquartiev mit mir zujammentraf, hat mid in ihrem 
66

fie afre nad ifrem Sittergutbe wollte, trennten fid) bicr unfere I $\mathrm{ch}$ in bem (Sebüfd berborgen, bis ith einige ehrbare Reifente gewahr würbe, um mit ibnen bollends zux Stabt zu gelangen."

Sie przăblte bas mit wahrbaft bezaubernocr Stimme; ihre blaffe slange fodien fith ein wenig zu röthen; ifr fonniger $B$ tiue verrieth etwaz von ber Doffrung, vielleidit in mir cincn chrbaren Neniden=

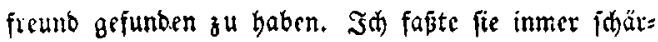
fer into Zuge. Fore saut, obglcith etrob gelblid, wat frin wie @eibe, ibre Micne ungrmein fittam und pinnegmeno, ifi SRut.b, mit joei Reifen bet

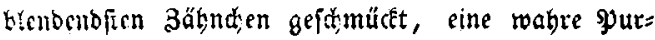

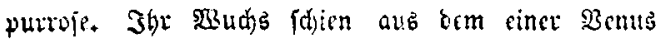
uno Diana zujamminiefest, uno ïr bantelbrauncs, frines Reitfleid verricth allerbinge vornet,me Scxtunft.

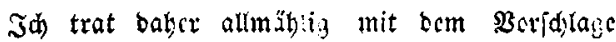
bevoor, fie in cigener פerjell nach ber Stadt zu brinisen, uno, fallz fie sort inre Barvanoten nidit fänb, bis zu anoerwertem untertommen itjren $\$ B$ c:

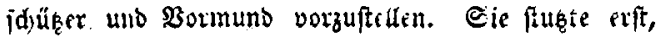


iditen mein anerbieten zu überlegen unb Elopfte bann in bie niebliden ড̧änbden. Stur bas eimzige Beben= len blieb ify übrig, ob fie aud bas fortemmen zls pferbe, bas fie lange nibt geübt, werbe vertragen tounnen?

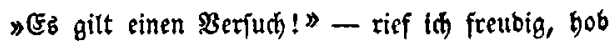
fie bann auf ben, etwaz wiberbarigen Rammblopf unb führte fie einigemal allf ber \$Biefe umber. Es gelang über Erwarten. Sh) muste ifgr baber nun felbit ten 3aum überlafien. Sie ritt nod einigemal immer fajneller und jodnelfer, in oer Runbe, lentete bann plöglid) ein, uno - jagte in vollem (Baflop in

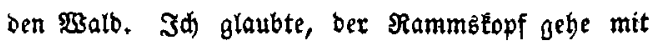
ibr burd) und rannte nad). Dod fie rief red)t luftig:

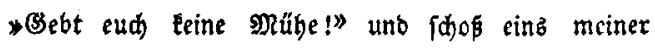
Diftole, boch nur in bic Euft ab. »Bleibt zurüce! ! rief fie bann, als id igr immer nod nadiferete *bleibt zurüte, fallb Euch Freiheit uno keben lieb ift! Euer \$ferbdien gefällt mir! 》

Nun war id) binfifttlid) oer fdönen Zmazone im Alaten, fpürte aber nid)t bie minbefte $q u$ ft, ocn Rummstopf im Stide zu laffen. 3 h verfolgte $b a=$ 
ber bie reizenbe 9 ferbebiabin immer tiefer und tiefer in bie Büphe, fo (d)nell id vermodte, bia id von orei bis viff biefer ehrliden Reute mit angelegten

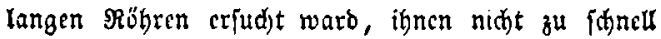
bав Bergnügen meiner (3)genmart 孔 entzichen.

Shr Eünnt lridgt crad)ten, alleroortrefflidglter Baccal uree! baj id) - geftalten Saben nat) - biejer freundichen Einlabung nit ungemeiner Erkenntlid) Feit Folge Xeiftete.

Doh muß iás ter Detila, her Circe, ber Arr=

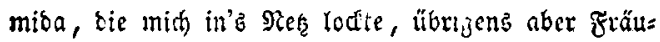

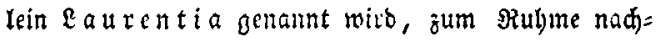
lagen, baf fie mid begen jebe Miphanblung in Sduk nakm. Sie fteht in gropem Refpect bei ber ganzen Rotte und nie Darf in ihrer Bezemuart eine Brau= famfeit verübt werben. Segr oft hat fie bie $\mathfrak{B} e=$ zaubten mit anjebnlidjen Summen unterftüçt, bie zu erwerben bem Doriften und ihr freitid nidht former werben. Ohne alfen 3awelfel bätt' id) aut) ben e:nft:

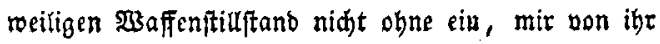
an Beorg mitgcgebenes, in wenio Budfitaben befte= benbeb seiden, unter fo billigen Sebingungen abges 
(d)lofen. Mogt \$br abrigens von mir urtheilen, waz Eud belicbt, unverbalten fen $E_{u d j}$, bos id nod jest

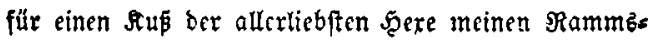
fopf, die mix abgenommenen (3)lder und $\mathfrak{B}$ unberarz ncien, ja, wenn $\in \dot{B}$ (r)n müpte, tiefen finger unbes

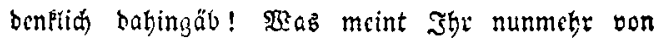
meiner Stugheit? " -

Die Erzäblung des ふoctorb, ber, wie id fpätcts bin oft zu bemerfen Bargengrit fand, bie Eitgenbeit bejaj, sm licbiten Befolidten zu erzäblen, worin er

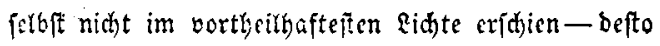
ungeftrafter fonnte er bagegen aud anberer fpots ten - hatte midh unb isn jelbit fekr erheitert, unb vies gieng logar in meine Srüume âber, als gegen Norgen bejdleffen worben war, ben nädjften Tag zu raften. Man fellte nun auf bas forgfältigite bie einftweifen Xubgeruhten zu Sdiřbradjen qus, bamit bie Uebrigen bor aller Befabr fidjer eine Beitlang fdhlummern könnten.

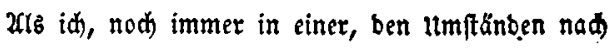
fchr fröblioben Stimmung erroadt war, wirtte ein Bits bes Sammers um fo fdmerglider auf mith ein. 
Denn, cine Zuböhe befteigenb, bemertte id biex Baucrn, bie gleid) SPferben an cinem $\$ p$ flug geipannt, in cinem abgelaffenen und jegt ouggetroteneten Xeidie aderten. So erbärmlit) mujten bamals biefe elen= cen Reute ihs Reben friften!

(Begen 2(nbrud) ber Nadt zogen wir weiter. Jh batte ein Roß betommen; ben Docior nabm Einer mit aufz פpferb. Die Gefangenen musten sefeffelt (id) burth Sacht uno Dunfel fortfinocn.

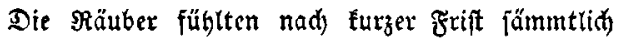
Ermattung uno bie Pferbe wolften nid)t weiter. Doh gelangten wir mit Norgendämmerung in cinen গ3salb, unb zogen nod zrbei Stunten bis zu einem tleinen alten Stäbtð)n, worin eine $\mathfrak{B u r} \mathfrak{B}$ la $_{\mathfrak{B}}$. Mit befielben Meier unb sBürgern lebte bie Mofclihar

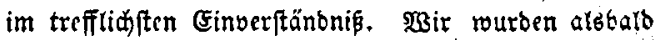
eingelaffen uno binter unz bie Ihore befaloflen.

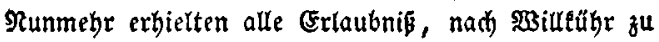
价afen. Die Befangenen fperrte man in eine Stube, beren Ihür und Fenfter mit $\mathbb{B}$ ad)en bejęt wurben.

Id) unb ber Doctor fhliefen febr feft unb exmun= terten uns erft Nadjmittags gegen brei ubr. $\mathfrak{I} n=$ 
beffen batte bet 2 wirth im gropen Saale bie Tafil auf bas Serridifte beftellt. Befottencs uno Ssebra= tenes, swilopret, Gseflïgel unb Fijáe, nebft ben be= ften Beinen, ftanben im gröbten Borratbe bercit.

Nad) Eurzer 3ait ftellte fin in biejem (Salthaufe aud Der Dorfwirth bon $\Re$, ein, berfelbe, welder ber tapfern (S) enofienjandt bie reiden Raufleute ver= rathen batte. Diejer erbob, bamit 2allez cin fein ebrbares Unieben behalte, grope Rlage, alb fey fein \$aus geplünoert und viet Gefdirr ifm zeridhlagen rorben. Er verlangte, bas man cinige ber ßeiter, bie er beffen befdulbigte, in $\mathfrak{B e r b a f t}$ nebme uno nad) Berbienti beftrafe.

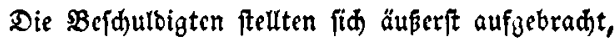
und rollten ihn nieberbauen. Dod firlen thre Streide fo fanft auf, wie Flaumfebern, und man folos in Furzem cinen Bergleid, bem $\$$ fak zroanzig Dutaten zu geben. Diefe Summe muste id, als Sdakmeifter, igm aus bcm gemeinen Säctel aufzăblen. $E_{\delta}$ war ber forb, obroohl ifn einer bes Raufleute nod beztudigte, zroangig Feidbthaler, welde er ihm in Berwabrung 
gegeten, unteridlogen zu haben. Dię rutbe jebort) nid)t genauer unterfudht, weil bie Mofelfidar $\mathfrak{e}$ ge rathen fand, biefen wackern Mann zum Freunde zu behalten,

Man madte fid Cie Racht burd reibrid tuftig, gegen $\mathfrak{z a g}$ aber ging mant wicber fallafen. Ith be= bünfte mir jekgt ein gar feiner Befell, mapen ia woht einfah, weld)en Eohne fith dirjenigen ga getrös ften haben, Deren Sonne ber Mond ift.

(segen \$ittag fam eine neue $\mathfrak{F}$ e lo t a ube, (b.h. in ber Felsiprad)e ein Bote) geflogen, mit einem $\mathfrak{B r i e f}$ Iein, baz in einen Erdeloß gepackt war, um ez im Faul ber (Entbeckung jogleid) wegfhleubern zu k̈nnen. Ser Brief war von einem Bogte befdrieben, welder lange 3eit in ber gröpten (sefahr geftanden, weil die Reiter, bie er einmal aubfpüren belfen, ifgm ben Iob gejdrworen hatten. Diefer rollte fith wiebex beliebt mad)en unb melbete in einem গauberwälih, Das Feiner redft ju beuten rouste, jebod) id) mit Şülfe bez Docturs nad) vieler gegebener Müh' ents zifferte - aud \$yancratius batte nun ein \$fero be= 
Fommen unb wir wursen beibe gang als Mofeler be= tradtet - ungefähr Folgenbez:

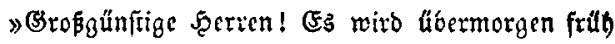
ein Sdjiff mit vielen $\mathfrak{3 a a r e n , ~ g r o p e r ~ B a a r j h a f t ~}$ unb ciner Menge æarাagiere von bier nad) Irier ab= gétil. Das ift 2ulke zu haben. 3u mchrever Sis herbeit fente id meinen Sobn als (Seijel."

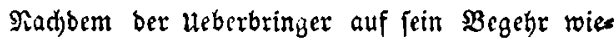
ber vor baz Iyor gelaffen worben war, um ben einftweilen in einem Barten ocrftedten Bogt5fohn bereinzufolcn, bradte er ihn in $\mathfrak{B}$ abjheit, einen gar feinen blonben und blauaugigen, etraab furditamen Snaben, ber, wie mid tief ergriff, meinem lieven, lang niá,t gejebenen jüngften Bruber aufb şaar glich. Man (pradh ihm Muth ein und fütterte inn trefflid), - bas aud idh hiebei war, barf id woht

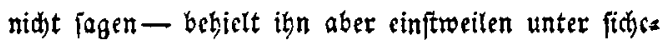
rex Dbfut.

Nun musten ifrer Reun tfeits mit langen, theils

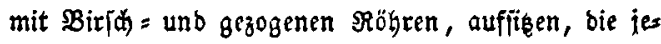
ber einen Infanteriften binter fid nakmen. $\mathfrak{3}$ d) abex

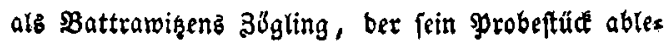


74

gen forlte, gab ben neunzebnten ab; Denn bie Räus Ler ftanben in ber Meinung, ungleide 3abl bringe

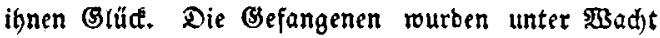
gelaffen und der Meier bez Stäbtdens zahlte für fie in $\mathfrak{B a u f d}$ und $\mathfrak{B o g e n}$ fünfbundert Reidzthales, um mit jebem (Finzelnen nad) Belieben sinen Zficorb

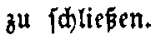

SWir hatten adjt frarke Meiten furückzutegen, fas Ben um zwei uhr Sadmittags auf und ritten in cinem Suge bie ganze Radt burd). Gegen Morgen Iangten wir in einem Stäbthen an, wo abermala volle Siderheit war, weit bie Befąung unz ans bieng. Şier brieben wir uno mađten unz luftig, biż die Sterne aufgingen.

Dann mußten bie Röprein wieber lostraben. unb wir ritten am ufer hin brei Meilen reit bis an ein völlig verlaffenes Dorf, wo wir uns in einer berfallenen Sđjeuer einquartirten. Die Feuerröbre nurben in ein (Gebüid) nake am Baffer verftedt, unb bamit aulleb befto. fidherer getinge, müten brei gu פferbe burd einen furth fegen unb fich am ent= gegengelesten ufer in sinterbalt legen. 
Xls nun am Morgen gegen adt Hht bab crwave tete Schiff ben Rhein berabidivamm uno bie orei, jenfeitz auflauernben Reiter fid) feken ließen, ftrengs ten fid ber Steucrmann uno bie Rubeter nad) Rög= liffecit an, auf bie anbere Scite zu grlangen, wo, ohne igr mififen, Der sauptfeinb auf fie laucrte.

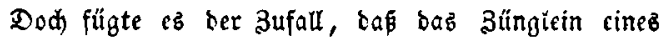

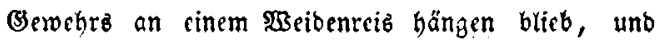
fonach ber ङdup losbranntc. Durd biefen Rnall twurben bie Sdiffer, bie fbon in bet Räbe anlanben wollten, unferev geroabr, uno ftiesen von neurm ine sibaffer.

Jest foboffen bie reiter am jenfeitigen ufer ihre Siftolen unb Feuerrögre ab, und tie armer leute zerarbciteten fith aufe ncue, in sie mitte uno fonad,

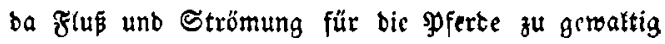
wav, in Sidjerbeit fu fommen. Dod nun wurte bon beiben Sriten wütheno auf baz Sditi gefeuert. Die ungliffliden Men[den, worunter fith aud Frauenzimmer befanben, berloren alle Befinnung. Einige fanten, von fugeln getroffen, 2rnbere ftûrz= ten, obne mebr auf bas Shjięen zu adjten, bin unb 
Ger, fhrieen unb jammerten, boben bie Şănbe zum bimmel. Das ganze St,iff, fhon an mekreren $D_{r=}$

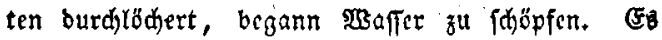
roankte hin und her. Untcr eincm furdtbaren 2 angfte geidrei gieng es mitten im tiffiten Strome vor une fern Xugen unter, $\mathfrak{R a n}$ jah lange 3cit nidhtz, atż rinige unb bas idnellere Nräufchn ber $\mathfrak{B r l l e n}$. Der Mafte baum mit ben ssimpel rititete fid crit nach ciner Tुeile langiam einige Ellen über bie flut empor.

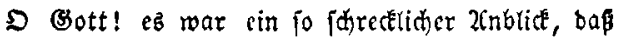
idh ifn, fo lang 2 them in mir ift, nitht vergeffen werbe. Daz şaffer wai gerabe nitht fegr unrubig, boh, bie obgebadte gurth ausgenommen, breit unb

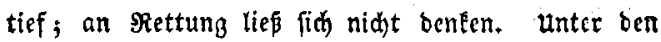
armen Berfuntenen, fünf unb zranjitg an ber $3 a b l$, follen fich, wie wix bürten, fehr wadfere und anger fehene Eeute, auh jum Iheil Xeltern bieler Rinber; befunden baben.

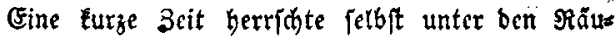
bern tiefe Srille, und nur nad) uno nad erbob fich tie und ba ein idfuer Blit. Beorg und einige 2the 
bere juirnen erfdŭttert. Die bei weitem gröpere Xnzahl aber Eümmerte fith in furzem soenig mebr

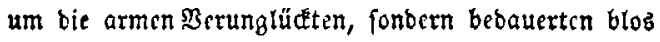

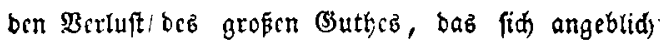

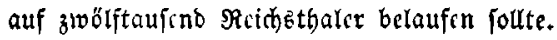

Diefe Stimmung warb bald allgemein. Der $\mathfrak{B e r}=$ brü über ben, wie mon lagte, fo lieberlid) aนร ben bänorn gegangenen Bortheil - benn man war ge=

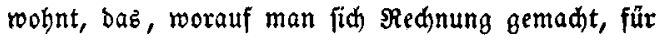
mit $\mathscr{F}_{\text {ug }}$ und Red)t gebörigrs Eigenthum anzujegen mad)te in $\mathfrak{i} u r_{z} e m$ Battramig uno einige der $\mathfrak{B o r}=$ nebmften fo unzufricben unb will, bas fic fid ocr= maken, nidht eher heimzuterhren, bis ber Sajabe ers

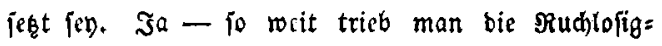
keit - man veridswur fid), ob aud (Einer einen im Şimmel unterzeidheten \$as hätte, ex folle nid)t un= angetaftet vorüber ziehen!

Run befand fich in bortiger Räbe ein Fllofter, bab, id) weip nidtt, aub weldhem Grunbe? zum $\mathfrak{E} u=$ therifden 2 bot genannt warb. In biefeb gelangten wir burdh Rift, unb ba bie Rloftergeiftliden fich in bie aufgelegte Sdaģ̧ung niđ̆t fügen wollten, wurben 
fie albabalb für mit bem seinb cinveritunten extęrt. Diejer Butwanb war, falls man ber löbliden Des

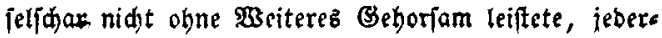
Jeit bei ber bant.

Man verfuty augenblidtid bem gemäß. Utı man Die Mönd)e zufanmen gelloppelt, wurbe alleb aufges idlagen und exbrothen, und, wie man luberidłlug, ungefähr bie \$älfte Der, burd) ben Sdiffibrud) erlits tenen (Einbufe mieber herbeigeidafft.

(Einer von bez 2 botz Dienern war burd) eine Xrt Eurtur zu dem Befenntniffe Bezroungen routen, baj ber Şauptidan bez Kilufterz unter einem Bebädt= nis̄lteine vergraben liege. Desbalb wuroen, viz man beficn Spur wirk:ich fanb - er betrug fünfzebnguns bitt Ducaten - gegen fed) mit sBilbroer verzierte Stcine aufgehober unb zrrijlagen, biebei aber jetbit ber Zobten nidht gefdont, fondern inre Gebeine her= aubgeworfen uno zerftreut.

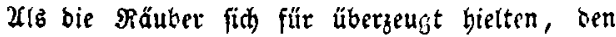

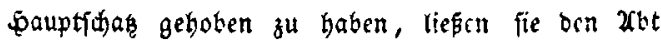
und bie Möndhe wieber fiti, um Effen und Irinten berbeizujdaffen, befien fid) jene auth nidyt vorigern 


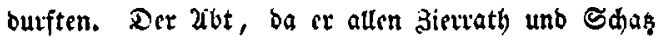
ser Firde zeritüt und geraubt jah, ließ jeinem 3orke freien \&quf und bielt eine gar eifrige, mit latei= nifthen Flobtern burdyebte Strafrebe, an ocrin Sthluife er jogar afgränen vergof*”). Die werthe (Sefellifhaft lad)te Darubcr, bas es forallte, unto bradite ihm cinen $b_{e}$ d)er auf bie Sriunbhrit bes Fubten $z^{u}$, ber ify die funfzeknhundert Ducaten ge= idientt! Da aber ber What und einige (s)eiftiche bon neuem filage erthoben, wurben fie, wie bie serren fagten, um bas פ̧faffengefdriei loz zu werben, fämmts lid) in bas (sünafel geiperrt.

Dhne unz um fie zu Kümmern, madten wir

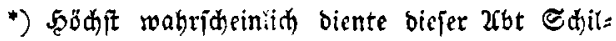
Yern bet bem Nöndhe in ten $\Re a ̈ u b e r n$ uno bem $(5 a=$ whiziner im \$allenftein zum \$orbilbe. (Eben jo leidt

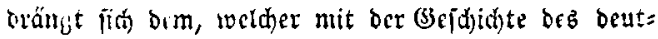

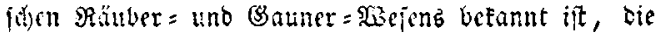

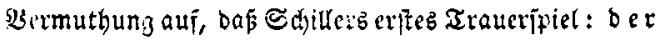
Stubent oon Rajfau mit beffen $(5 a r \mathfrak{l}$ : oor jirmlid cins und bajielbe gerveien forn möge. Bgeis

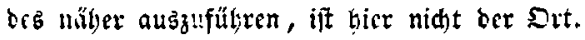


80

uns, ba fid) Mrunn und Roß gejättigt, mit ber rei= den Bcute wiebcrum auf zur Saupt = (Sejellfhaft, allwo wir bie Gefangenen nidt mehr vorfanocn. Die maiften batten fith mit bem Meicr um bas 2 ubs= löjungsget verglid)en, cinige waren entwijd)t. Der Meier foll bei bem Menidenbandel adthunoert Rcidzthaler bcrausgeprefit, folglidy oreibumbert rei= nen (Gewinn gehabt habcn. Mab er'z bereinft bei Gott verantworten!

Rummekr warbe ber fum unterpfande furüdbe=

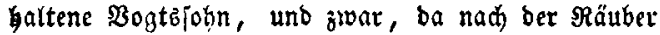
Urtbeil jein Bater an dem Berluft bez Shiffe ja mid) unr [ad)e gewefen, nebft ciner Belobnung von zwollf Ducaten, bem Boten roieber überliefert. Эa) Eonnte mid) nidit enthalten, ben lieben rothwan= gigen, immer nod) äng(tid den Sinaben, alb wär ex

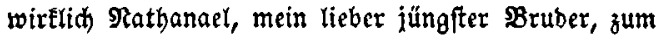

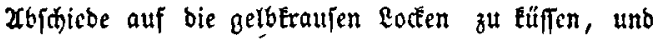
ifm babei im Stillen cin Segeneivort auf ben $\mathfrak{W e g}$ zu geben. Er bünte mir unter biefer Notte ein Eamm unter Bsiern, ein Engel, ber in ben Sdroe: ferpfuhl gefanot worber. $\mathfrak{A}$ th! was connt er füt 
Feinen, nur auf eigne Siderheit benkenben Bater? Dhne beffen frhänbridje Berrätherei wär bą un= blüc nimmer bejdeben!

Die Befellfwaft raftete brei bis vier Zage unb verbielt fid) gar ftill, um bie Cimwohner burd ben Brauben, fie fey fort, fider zu madhen. Zuth wurbe bieß (Serüd)t burd beftod)ene \$Bauern verbreitet.

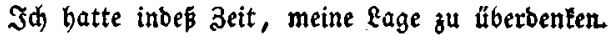
Der Untergang beà, Sdjiffz fam mir nidjt aus ben Bebanlen, und wenn id) in einfamen 2ugenbliden bas Birb meiner fanften $\$$ ghyllis betrad)tete, fonnte id) mid) faum bar abränen cnthalten. Szätte fie getwust, in welder (stejellidaft id lebe, waitbe fie nitht igr serz von mir gewendet, wärbe fie midh nitht verabjacut baben?

Der fdlaue \$pankratiub morte meine (Sefinnungen sum aheil errathen, mir jebod), weil id bei ben $\mathfrak{I n}_{n}=$ fühtern wohl gelit:en war, nidht volleommen trauten. (Er madte fich baber bei guter Belegengeit mit ber frage an mith: : Iid) fen, baß fo vtele wacfere Ecute in berfelben Stunde unb an Einem Drte bătten fterben müfifen, 
ba fie bod ofne ąreifel nicht biefelbe Beburtzftunde, nod) baffelbe Şimmelazeichen gehabt? ba id jedod meinen Glauben yon ben 2(jpecten burd) bie $200 r t e:$ "Astra iuclinaut, non necessitant" - die man wohl verbeutiden könnte:

》Sey bu nur fromm unb bete gern,

So idjaben gar nidtz bir bie Gtern'! —*

zu exkennen gab, lies ex ben Streit balb fallen, ging nuf unjer eignes Berdjid über, uno rüdte zulek̨t mit feiner wahren 206 fid)t heraus. SBSir verbanben unb nod) weit inniger unb berebeten unz, bei ber erften (Belegentheit gemeinjhaftlich zu entfliefen wab benn freilid) leidter zu bejdyliesen, als auszu= führen ftand.

2Cm fünften Iage fam abermalz ein Bote; bent

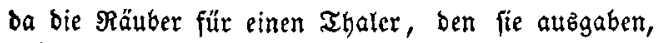

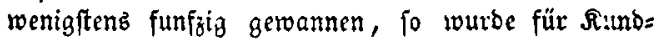
idjafter Éein Gelb gefpart. Ser jeşige trug einen grünen (Eid)en=-3weig in ber band, alb rolle er bamit bie Müten abrehren; eb war aber zroijden zwei Blätter beffelben mit grüner Scibe cin Brief= lein eingenähet, baz id, wie geroögnitid; lejen mujte. 
Der Inthalt babon war folgenber: \$ Der jawarze Umtapadter in ber Eleinen Stabt mit bem Rang= (d)nabelthurme und ber groṕen אirche, ackert morgen

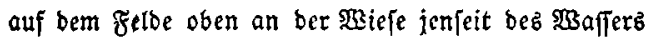
mit vier פferben und fünf Dhjen. Er hat zwar fieben Mann mit Feuergerwehren bei fith, aber bie

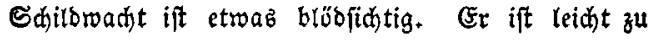
fangen, ehe nodh bie spferbe anz Geftab kommen."

Dieje Radridht war ben Räubern boppelt will= tommen; Denn ber fogenannte forwarze parater wat von töjen Reuten ber sande alb ift Feind angege= ben. So jafen benn (Filf um Mitternatht auf, um zwei Meilen böher hinauf über bas Finer fiteg auf sine bobe $B$ buthe; andere trodyen bin= ter cinem ב̧aune weg, um alles genau in 2 ugen= fdein zu nehmen und zu red)ter arit bas aeighen zum 2ungriffe zu geben.

SSii andern ritten nod) etwab weiter und ieģt erft warb id inte, was freitich wegen bes vieten

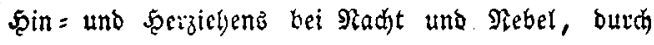
Didt uno నün, zum Theil auf Sdlcifwegen, mir bie jeşt entgangen wax, bnß ich in bex sähe meiner 
$8+$

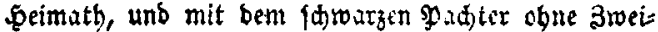
fel einer meiner liebften Sugenbbelannten gemeint fey. Es war in ber That fo, unb bod tonnte id bas ibm brobende unglüd nicht abrenben. 2uh lei= ber! wer unter פ̧zölfen ift, muß mit beulen. Dod babe weter id, nod) ber Doctor, obrohl wir für Ditglicber ber (Gefellidaft galten, auch mandimat Belb crficlten, bie ganje 3rit líbre, wägreno wir unter ins verioeilen musten, nie einem Menfdien an \&eib unb Reben Sdaben zugefügt. Id war fo zu fagen, Geheimfdreiber uno felb = (5afitrer. Banca=

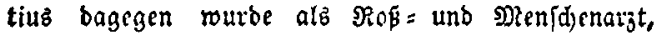
zugleid aber nebit einem gemijen $\mathfrak{z}$ affal, einem rothbärigen, feiften, großjprebserijd)r und efelhaft

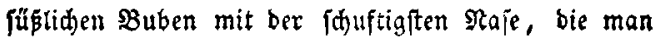
je gejeben hat, zum SPforbedicblable gebraudt. Dan fubte er, theils aus innerer lumuhe, theils noti eins gebent feines thcuren geraubten Fammstopfe, theils audi) bem $\mathfrak{E} a f f a l$, ben er nidjt ausfteben fonnte, zum $\mathfrak{P o f f e n , ~ m i t ~ r e h t e m ~ B e r g n u ̈ g e n ~ a u b , ~ u n b ~ f a m ~}$ feinem Ditwerber oft zubor, ober ftabl iam roobl gar bie geftoblnen spferbe. 
Der fhwarge Yachter fubr zur angegebenen 3eit

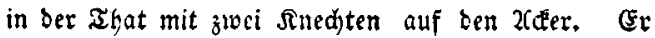
Gatte Sdilbrodjen, bie auf Bäumen fápen, un fiesen Dubfetierer zur Betecfung * Er jelbft roar mit

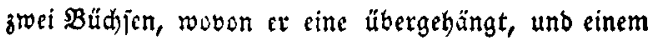
Fäuftlinge*) bevafnet, Mit fo groper (Sefahe mubs

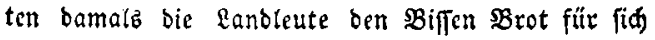
unb itre Rinder erbauen.

Sobald bie Reiter bemertten, daß bie eine ber

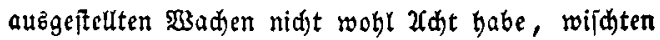

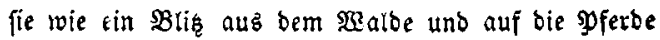
z̆ Diefe rurben ifnen aud, ba bie Xderfmethte, bem $\mathfrak{B e f e b l}$ zuwiber, auseriffen uno nad) bem Stäbt= ben jagten, einer berjetben aber zur Erbe fiel, nebjt ven Ddjen zu aheil. Der fdwarze spachser roar

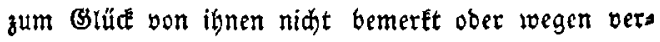
änberter $\mathfrak{A l e i b u n g ~ n i d y t ~ e r f a n n t ~ w o r b e n , ~ u n b ~ e n t f a m ; ~}$ anfonft bätten fie fidjerlid licber bas 2fakerbieh in bie Sdange gejhlagen, um ihn felbjt fortzuführen unb, wie fie firib vermeffen, in Stücfe zu gauen.

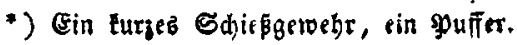


S3iv zogen mit ben erbeuteten 9 ferben und Stie= ren bavon - jo gern id cin wenig geblicben wär; an cin ltrlaubagefud burft' ich, wollt id nidht ver= bädtig verben, nicht benfen. Der Bote, ber li.tb geführt hatte, befam zur Berehrung sin altes Iab=

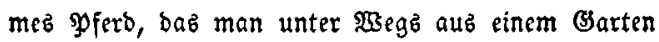
hatte mitgehen heipen.

शुar übrigens biefer Streid auth in fo toeit gelungen, fo fand bod bie töblid)e Sohar uriache, ibn hinterher zu bereuen. Wir glaubten uns nämlid, nadjbem wir bis gegen sracht immet fcitwärts gezo= gen, in Sidherheit, uno madyten in einem abgeleges nen Dorfe \$alt, fo wohl um ben eignen Sunger und Surft mit Brot uno uno noh mehr, um cinmal die spicrbe zu fíttern; bing bod von biejen gröstenthcils unjer seben $a b$ !

Man ftellte, wie ftetz (Bebrauh, sine Sdild= wad)t vor ein Şaus, uno machte mitten in ber Stube Feuer an, bap ez burd die zerfallnen răben fhimmerte; Fenfter waren in Dörfern feit Jabren nidt mebr zu finben. Sennod fdienen bie Räuber nidht red)t zt trauen; einige fprahen beimlid; bie 
smannidaft verfigte fid nebft ben פgferben in bas vierte 5aus weiter, uno alles mute fich ftill galten.

Die Räuber hatten faharfe Bitterung. Denn einige $B$ ürger uno Anchte aus bem Stâbtlein, wo fid) ber fawarze parhter aufhielt, unb ber fedifte

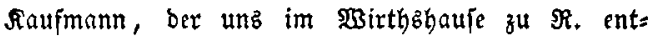
wijdte, hatten fich berebet, bem geraubten Bieh nad)zu[eben, unb famen, mit zroanzig freuerröhren bewaffnet, ungefăbr 20bends um neun uhr auf unire Epit.

Sobalo ber Şachpolten ifrer anfichtig roarb, machte ex Rărm und that fur Eojung sinen Sdup unter fie. Sene blicben nidits fohulbig und feuterten sann fämtlid in bellen Saufen burd) die Räben bes exiten \$aufer, in Meinung, wir befänden uns barin.

Die Sdillowahe, ciner ber brauften Eijenfref: jet, warb getöbtet. \$Bit aber, burdh bas Sdjiejent fattfam geroarnt, fd)lifpften zur Sinterthür hinaus; warfen uns auf bie spferbe, jagten hinter bem Dorfe herum unb fielen mu ben Zugreifern, ehe fie wie:

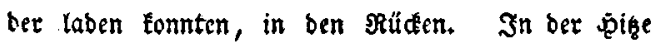


88

bes Xnfalls wurben fieben berferben niebergefdoffen, unter biejen ber Raufmann. Эa) felbit batte in's Blinbe in ben Dampf binein mit gefeuert; benn hier galt Eein Feiern; bier galt bloz: Friß Bogel ober ftirb! Fünf anbere wutben gefangen; bie übri= gen famen, ba bas Dori ganz öb unb an vielen Drten mit (Gefträud übermad) jen war, glüdilid) ba= von. SEir blieben bajelbft bis zu Tagzanbruda. Die fünf Arreftanten, worunter fehr wohlbabende Reute, wurben gebunden und geroögnifider Maajen fortge= trieben.

Unter เoas für graufamem Diäuen, Fłudłcn uno Edflagen biejen nun zuförberft für ben erjdoffenen Solbaten breihundert Ducaten als \$äăhrgeib abge= fobert wurben, will id gern mit Stillidweigen über: geben. afrs Einer von ihnen fidh bej halbftarris meigerte, mit bem Uuebruck : 》fo viel (Belb für fold

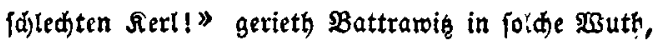
bas er ith auf ber Stelle nisberbieh. Swei Indere musten, bamit gleid)\{am ein siriegbredit gehegt

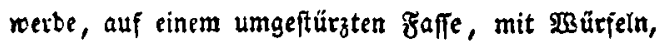
sie einer ber Solbaten aub ter Tajte zog, um bas 
Reben whiffeln, und ber ez veripielte, wurbe obne Erbarmen erfdidoffer.

Kud will id ber nadfolgenben Selbenthaten, bie,

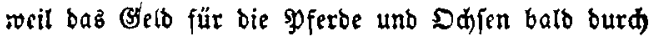
bie (Surgel gejagt rar, aufs neue unternommen wurben, bier nidht gedenten, wohl aber, das balb barauf ber Bande, abermalz burd) Berrath, , vor

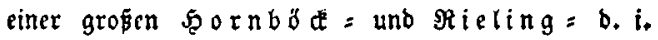
Rinber $=$ und Saneine $=$ ફeerbe breibunbert und zwan= jig Stück in bie Şänbe fielen. Şievon tourben albz= barb einige gejdlagen, geftodhen, verihenft und, ofge

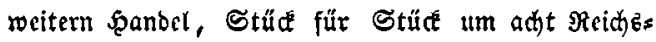
thaler verfauft. Den übrigbleibenben, bei meitem größern a a heil jollten bie șändler mit brittebalb tau= fenb İhalern austöjen, weldeb (Selb aud) nad) breirn Iagen erlegt warb.

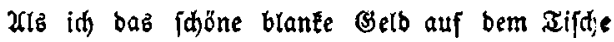
aufgezählt fah, jagte id auz unzeitigem Sd)erz:

》2⿰亻⿻ du lieber (sott im scimmelreich, S5sie theilit su bas (Suth fo wenig gleid);

Du giebft fo viel oft Einem Mann, Daf vierzethn Dieb' Gätten gnug baran! 
90

2ub unbebadt ließ bet Biebyänðler fich ou ber 2 nt: wort vorleiten: " (FE fino bod) ber Seerren nid)t vier= zefn bier, idh lehe nur neun! « Şierüber ergrimmte

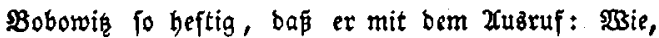
Rerl, bu băltit uns für Diebe? Rerne Reppect vor tapfern Solbaten! " feinen Faufthammer zudite unb auf ben seänbler los wollte. Sh fiel ibm in ben Xrm unb bat vor, weil id ja jelbft ben armen Mann berführt, er's audh gemis niđt fo bób gemeint bätte. Sierauf wollte inm Boborvis zur Strafe folner Eafferung wenigften bas Eoffegeld bchalten, ofne bie Seerbe berauszzugeben; allein (Seorg, ben if berbei= rief, toiberiezte fidh bem.

Diefer hies nun freilid ber Sobrifte; bennod gab, wie eb unter bergleichen Worben zu gehen pflcgt, zu Beiten Sciner viel auf ben andern. Dephalb wollte Boborvis nid)t unredit bebalten uno ber Bichbänbler verrieth uns nun, theile um leidtern Raufa babon 3u Fommen, thrilb aud) aus $2 b_{\text {gunft }}$ und Brooneid, cine Seerbe fedzig feifter Dhjen uno Rübe, bie in einer Entfernung von ungefäbr zehn Mailen burchs Lanb ziehen werbe. Dafüt erbielt er cinen Radjla 
von zroeifunbert reidatbolern, muste jebod, weil man befürditete, cr möhte audh an uns zum Bers răther toeroen, annod bablitien.

2(m vierten Morgen, als ber Biphbänbler nod) fahlief, madten fidh unferer funfzenn zu Soferbe bavon. Sair ritten ben ganzen Sag uno die ganze Rad)t, auper baj woir bes 2 abends in einem Bewälo Brot, Galz und Rnoblauch fpeiften und Tabad bampften.

Darauf ging es wieber fort. $\mathfrak{B i r}$ gelangten zur iedten 3eit zu ber Matte, wo ber erbaltenen 2 w $=$ zeige zu Folge die Shjen geveibet werben follten und, bamit id's furz madye, nactbom wir lange in Beץorgniß geftanden hatten, ber Streid mőge mifs lingen, warb eincr ber firten erfajofen uno bie ganze Seerde erbeutet. Dic Räuber fanben, weil ber Sandoerfauf bas vorige Mal viel eingebrad)t batte, für räthlid)er, bas angebotene Röjegerb nidit anzunebmen, uno ba bie Banbe auf 20 egen, wo fonit Ecin senid) hinkam, io gut Bejdeio wufte, ats 2unbere im eigenen Seofe, fo bradten wir mit Shülfe ber gefangenen בreiber 2alles in Sidherbeit.

Rummehr wars aber auf allgemeines :Berlangen 
92

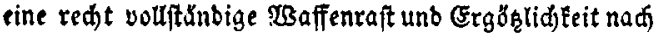
to mandjerlei Strapazen bejaloffen. Sie 2unfübrer fanbten nod mäbrend bes sarjdes nad) (Samera ben, Gäften unb Spielleuten veitcnoe Boten auz.

Diefe Einlabungen waren von bem beften Erfolg. Denn eines Mtorgens, als wir ohngçähr biz auf zwei Mcilen zu bem beftimmten Raftplake gelangt, vernabmen svir, plökli(h) hinter cinem Sügel bervor= fdalleno, bas gar luftige Irompeterftücétsin :

》) S\$zaz helfen mir taujeno Ducaten? « unb Mebrere ber (Sejeldidaft erboben ein Freubens

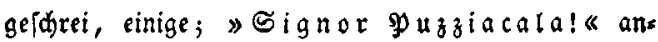
bere: $\gg \mathfrak{r i n z} \mathfrak{A} \mathrm{mabi}$ ! ! «

Diep erregte meine seugier uns id gerwahrte einen fonberbaren 3 ug, aus bem \$̦ohlweg herbors fommenb; Reiter, zum abeil blajent, audi) einige bedecț $\mathfrak{i r a ̈ g e n . ~}$

Drr 2rnfübrex jenes నruppb, ber auf einer gropen Falbe, wie weiland Silen, wadfelnd ritt, (dien nod zu ben Enatztinbern zu gehören. Sein ganz tables

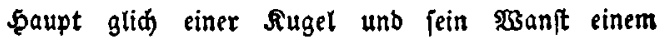
\$3allfifabaude. Dazu batte er hinter fid eine grofe 


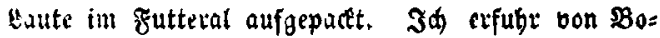
bomis, ber neben mir ritt, ber fettwanti fey ein

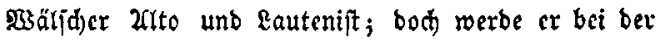
शrofelfd)ar, wegen fciner Sđwerfälligleit uno facig: teit, nut alz $\mathfrak{B o r f t e h e r ~ b e r ~ f e l o m u f i e ~ u n d ~ z u m ~} \mathfrak{B}$ er

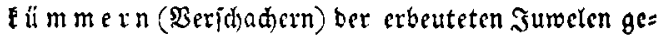
b:audt. Eine rocit beffere Figur madyte Junfer 2cmabis, ber einer ber Bornehmften fdien uno in Eurzem bem 3uge boroubfprengte. Diefer, ein fibüner falanter Sdhmarzlopf, tem cinige faft gleidhe Bärtlinge folg= tin, ritt auf cincm frattliden, prädtig gefattelten Dferbe; pr war in Sammt, Seibe und Bold gethe bet uno mit sinem feberbutc gridnualt. Er jomogl, aib orei ofcr vier feiner seg!citer, gliden ganz Gabalieren, zu irgend einem 5offefte ziebent. Dar (f)ienen fie Nuttcriöhnden, bic, bur(h) alfu luftige Eebensart unter bie (sejellid)aft geratben, nun etroa bas Befte berfelben burd falfdee 2 ürfelfpiel ober Spionerie in Dbad)t zu nebmen bătten.

Urs man fid gegenjettig genähert hatte, iprangen siele bon ben Syferben. Man beroilleommte fid alf's freubigfte, man umarmte fí aufs brüberliфfte, 


\section{4}

und feste fich bann wicber auf, um gemeinjd)aftich bie Reife zu vollenben.

Mir fam eb oor, als fen id bem fogenannten Xmabis aufgefollen; aud) bemerte id balb, baf er bei Georg fid nadh mir erfundigte. Diejer rief midg und ftellte mish bem JunEer als einen (Gelahrten vor,

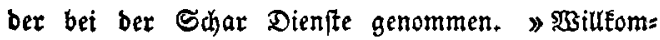
men, taujenbmal willémmen, \$̧err Bruber!》 rief Ladjenb Seerr Xmabis mit bellunkelnbem Blice, in= bem er fich bas farwarze, glänzende Stub̧bärtlein

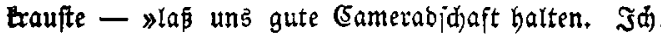
wär aud noth ein blober Feberbeld, hätt' ich nidht cinen Eameraben auf bem Tanzboben erftodyen!》

Şierauf bieß mid) ber Sunker ihm zur Seite bleiben uno erwies fid fo geroogen, daj id mir die Freiheit nakm, eines oritten Nujenjobns, näm= lid) bes Doctors Ermábnung zu thun uno ihn her: beizuwinken. Sanciatiub aber fijütelte gewaltig mit bem Ropfe, verzerte fein (Sefid)t, alb hătt' î́n tine Iarantel geftochen, uns fog fid unter ben stad)= trab zurüd. Jd) mufite glauben, er jey mit 2 ma $=$ bis jon berannt, bod ctroas vericinoet, Der Jun= 


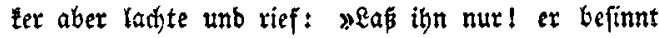
fid) (d)on eines 2undern. "

2Cla wir endlid - etroa früh netm $\mathfrak{u} \mathfrak{h r}$ - an Drt und Stelle, nämtich in eincm großen (Safthof? angelangt waren, wurbe jeben ertaubt, ber Ruthe

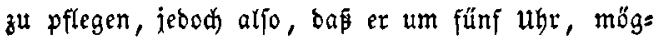
H(d) geläubert, bei dem zut haltenden Banquet fiij einfinde. 2(ud) erbiclt, wer ę verlangte, neue Rlei= bungbitüde, wovon eine ganze wohlverjehene Mon: tirungblammer offen ftand. $\Im$ dh unb bie Meiften Golten fich bort, was fie roouten und beffen jie größs= tentheire jebr bebürftig waren. Dann zerfteuten wir unb und fuchten, wo es fid) in ben bereit ge= Galtenen Baftzimmern eben fand, ein Rubepläkdjen.

Sur beftimmten 3eit war id) wieber munter, gefiel mix in meinem neuen feinen 2Unzuge, beffen fid tein vornehmer. Sifficier zu jhämen uriade gehabt, vor bem Spicget recht roobl, verfügte min nah) bem Speijefaale und traf an ber:Thtir auf panfratius. $\mathfrak{D a}$ ich inn fragte, was irm ber bübiche $\mathfrak{E x}_{\mathfrak{x}}$ S Stu= bent zu Leibe getyan? erwics er jeiner Najenjpige 
96

bie Berooknte Égrenbezengung, meinte, id habe ben Staar, unb fiob mid in ben Saal.

Эđ) prallte vor Erftaumen zurüct; benn an ber wabrhaft fürftlich bereiteten Tafel jąen bereite fünf bis fed)b, ganz roie Ebelfrauen gelleibete Sdönen, obon an abor, alb bie prärtigit gepuste, bod isęt ohne Stugbärtăen, Junfer 2umabiz - in rociblīer Zradt Fräulein Raurentia genannt!

Row wie geblendet rief id bem Doctor ine Dhr, was Soib von ber reizenben Sägerin $\mathcal{U}$ tarante fagt:

„Facies, quan dicere vere

Virgineam in puero, puerilem in virgine possis !" ") und Fonnte mir nun fattfam erlelären, wie ex bei feiner Sdrväh) für baz meiblid) (3)ed ledit, ber ein= zigen, wovon cr fidh nidht befreien Eunnte, von einer Eva biejer 2 rt fíd batte berfübren laffen.

Es war in ber That - nod jeset feht Raurentia's

*) Eir (Befitht, jungfräulit) bei einem Săng= ringe, jünglinghaft bei einem säbden zu nennen. -Or. Metain. VIll. 322. 
Santalige Eridheinung lobbaft bor meinen Xugen -

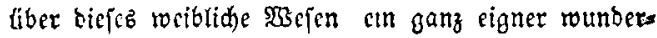
barer 3aubcr verbreitet. (s)ich fojon ihre Farbe weniger bem 2labafter, alz cinem gelblicten Mass mor, röthete fid ihre blaffe \$̧ange gleich nur jetten

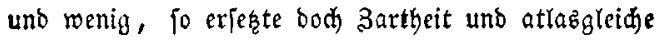
Feingeit ber Szaut, bie bödfte Bollenoung alker For = men, ber frifhe purpur ber Rippen und cine gerwiffe, taum bejdreiblidhe, id mödte fagen, rilbe Sdöns

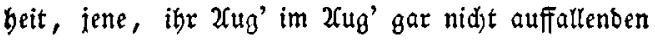

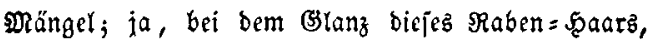
Diejer gropen, tief jojmargen unb bod unter ben fein gezogenen Brauen gleid) Riebesfareln ftrahyenben 2 Cu= gen fdien eine füblidie Scautfarbe faft ben $\Re$ Riz zu erböhen. Man benke fith babei eine fhlanke, unge= fäbr brei und zwanzigiährige Beftalt in bem, wohl=

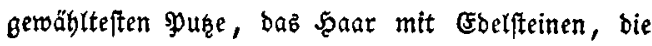
Bruft mit \$orlenfd)nuren gejdmüdt, benfe fie fid mit 2Cnnuth, einnehmender ફ̧eiterkeit uno lebbaftem Beifte aufb reidffte aubgeftattet - unb man wirb

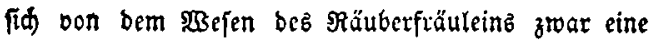


98

ungefäbre, bod binter ber \$äabrheit immer nod zurüdbłeibende Borftellung mad)en fönnen.

Zfud) unter ben übrigen Frauenęperfonen, welde für Semahlinnen unb Sdyeftern verjdiebener von Den 2nfübrern galten und größtentheile ale Dfficterg: Frauen titulixt wurben, waren recht artige Creatu= ren, alle kőfftid), zum Iyeil auch sin wenig frei Befleibet.

Daş rabenlodecige Fräulein batte das $24 m$ ter 2Birthin übernommen unb wies bem Soberften Beorg Den plą̧ zu ihrer Redten, mir aber ben zu ihrer

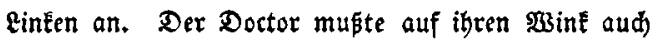
in unjerer Radbarjaftaft bleiben; ben übrigen Theil ber Iafel umgaben, möglidjt in bunter Reibe, bie andern Genoffen, weldhe fich nach und nad, alle bod)= zeitlid) gekfleibet unb zum atheil aự mit Frauen, verjammelt batten. Dab (Banze glid) bem (B)aft= mahle eines (senerals in einer eruberten Stabt, uno fidwerlidy würbe ein Fremoer, ber hereingetreten, alsbalt vermuthet haben, in welder Bejellidaft er fith befinde.

Sunmebr begann ber Sdimaub, wie itgn faum 
ber reidhe $\mathfrak{R a n n}$ in Evangelio glänzender auggeridy= tet bat. Die, von \$uzzziacala fommanbirten Spiel= leute, fünf ober jedhb an bex 3ahl, liesen fid $\mathfrak{n} a d$ h Bermögen, hören; Irant unb Speife in $\mathfrak{u}$ eberflú verbreiteten allenthalben Freube und $\$ 30$ glbehagen,

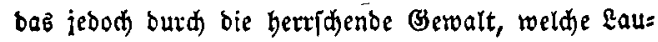
rentia mit Berwanbtheit und 2 rtigkeit aubübte, ziem= lid) in ben Sdyranfen beb 2 ñtanber blieb.

Bermuthlid) um bieje feine äupere 3udbt aufied)t zu halten, fahlug bab Fräulein beim britten (Sange bex (Seridjte vor, etwaz zu fingen, uno \$yuzsiacala, Der fib gewiffermajen für ihren şoffänger anjah, lies

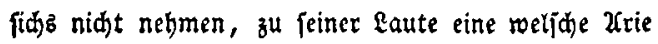

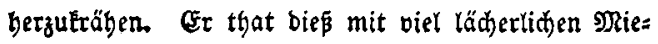
nen und Bewegungen, fo das ben Meiften bas, was fie nidt verftanben, bow getwiffermafen begreiflid warb, und endete erf, ats er gervaltig folwighte unb keudte.

Nunmebr foberte Eaurentia - id will es nur belennen, mit einem Briďe unb leifem şänbebruďe, ber mir, batt' id) gleid vor kurzem beim umeleiben

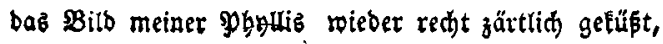


boh) burd) bie Seele futgr - mith unb ben Doctor, bod) midi zuerit zum Bejang atis bem Stegreife auf.

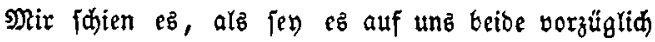
gemünzt. Jh) rax zu fehr in Bervirrung gexatben, um biefem $\mathfrak{B e r l a n g e n ~ f o g l e t c h ~ F o l g e ~ z u ~ l e i f t e n , ~ u n d ~}$ madte $\mathfrak{s}$ baher geltend, dak dem spancratiub, als Doctor, folglid) aud) als bem (selabrtejten von uns Beiben, ber Borrang gebübre.

Diejer batte zwar anfänglid Laurentien mit feinen Błidén f(hier, wie bie Mücte bas siđut, umgautelt, fdien fid aber, wie er Denn überbaupt leidyt luftis warb, ja ber allerluftigfte unter unb 2 Ulen, bei feis

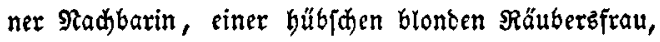
redit roogl zu befinben. Indes, von Raurentiens feurtgem shlic getroffen, veriprad) er joglcid) zu gehordien und hatte bie unverjäämtbeit, zuerft

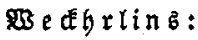

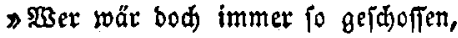

Das bei bem lieben Rebenjaft $2 c_{*} \approx$ unb, ba bieß 3ed)lied Bcifall fanb, Spitzen 8:

\d) empfinde faft ein Brauten

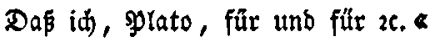


ganz, alb bidgte ex aub bem sopfe, vorzufingen. Sid verbroß cin wenig, bie ibm allgemein, ja jelbft bon Raurentien, gezollte Berwunberung, zumal ba pancratiub ladeno verfiderte, to etraz fib ithm ein Spicl!

İ) niefte baher einigemal vernabmlich unt erbot mid, ce bcm Doctor nidit allein gleid), fonbern nod) vorzuthun. (Ex zog dieß in 3̂weifel und id begann mit lauter Stimme:

\2uf! gehet tapfer an, the meine Ariegegenoffen! Sdilagt ritterlid barein; eu'r reben unverbroffen Für's saterlano auffegt -

"Profioiat!" rief mir pancratiuz zu, alb beant= worte er mein vorgeriges siejen, und wanbte fich oann, fect vertrauend, id werbe eb hier nid)t zut eincm Sdulilgezănt fommen laffen, wab wohl Einige

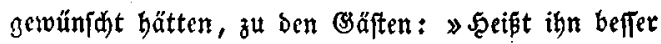
fingen, ihe Serren! Daz sieb ift bon sinctgrăf,

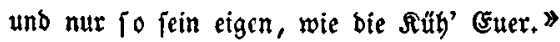

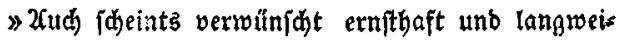
li $_{B}$ ! - fielen (sinige laut ladjenb ein. - 》 Sing 


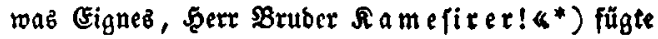
Eaffal bummbreifi binza.

Theils ärgerlid über bez Doctorz Rühnheit, theils um înn zu befdämen uno von meiner Fertigteit zu überzeugen, theils aud), um für bas ertheilte Ehren= wort Dank zu fagen, fang id alabals aub bem Sopfe:

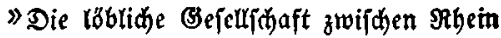
unb Dofel liebt baz $\Re$ iüitigienn;

Sad) unfall fie nidt fragen;

Dab (Erbreid) bin und her, Die Rång' burd unb bie হucer, â Fuß und פyferb fie jagen, Frifd $\mathfrak{X}$ Ulez wagen, Rein Sđeucn tragen.

ueber hohe \$erg', durdb tiefe Ingal Fall'n fie baher wie Blizzesftrabl, 2curwegs ohn' :şeg fith finden;

*) $\mathfrak{R}$ a mefirer, nath ber Ferbfprade: ain ver= laufener Stubent. 
SBei näd)t'ger Suntelbeit,

şann [कlummern anbre \&eut',

Sie zules fein aufbinben

Shn Riditanzünben,

Ridhts bleibt bahinten. "

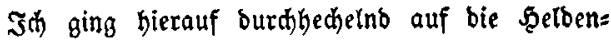
thaten \$oborvikens, \&affals unb \$puzziactla's über und jolof :

\$ (ehts in ber

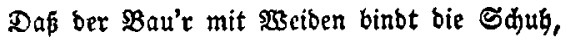

und uns bie 3ed' muß zahlen,

So Iang' er hat ein' אuth

Die Röß̈lein aud) bazu -

Die Rappen mit ben Fahlen

Sm Sdhlund wir mablen -

Der Bau'r mú zahlen!

Dies Stücklein, fo wenig werth es ror, warb mit lautem Saut)zen aufgenoinmen unb felbft saffal und ber fette Rautenift, berer id am wenigften gefdont, musten bei bem fhallenben Sclädter igren Berbrus unterbrưcten. Man verlangte co nod) cinmal, jang eb nun im shor unb nidt allein jene, jonbern jetbjt 
104

ber Sd)ulttheip dez Sotta, der die Einlabung zu biejem Schmaupe nidjt auszufdlagen gerwagt batte, muste einftimmen.

Dafür warb leģterer fofort auf ben nädfften $\mathfrak{S} a g$

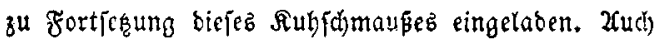
bejdlos man, theils um fich befto beliebter zu maden, theits um jein $2 \mathfrak{n}$ iehen zu zeigen, bie übrigen Bean= ten ber Stadt, bie Befehtababer ber fleinen $\mathfrak{B e}=$ faģung, weldhe bereits von ber gcmadten Bcute eine Berebrung erbalten, to wie fonft bie Bornebmiten baz̆ zu ziehen.

Das Singen, Sdhrcien unb Rärmen, aber aud) bie Irankenhrit, nahm nun immer mehr unb mehr überband. Da felbjt (Beorg fid berfelben biz zum uevermaaz überließ̄, uno id) uno Raurentia bie mei= ften ber angefitmmten Ricber nidyt mitfingen modten, fo führten wir indes mand)erlei (Sepprädbe, und an

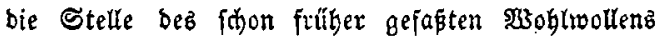

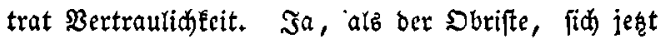
wenig um jie kümmems, aufitand und mit überlau= fendem Blafe unter ber Menge berumtaumelte, gab nir bas Frg̊ulein ben $2 \mathfrak{r m}$ und verfügte fin mit mir 
in einz ber effenitefyenden Nebenzimmer. Der Docs tor, ber biép bemerkte, fam uns, fen ç nun aus SBerlieftheit, ober aub Borficht, in furzem nady.

Da wit to zu brci, ziemlich froben und aufge= wecten Muthes, beijammen faß̧cn, fano id) fein Bedenten, bem zräutein meine Berrwunderung zu erecnnen zu geben, daj fie, nidet blop von ber $\mathfrak{R a}=$ tur, fonbern aud) allem 2 nnid)eine nad) bom (Blüct felde begúnftigt, zu bem Eeben unter biefer Genofiens (d)aft fid entidlofien babe.

In wie weit ez igr nun am şerzen lag, fidh in meinen 2 ugen 孔u redtfertigen, bleibe bahin geftellt. Genug, fie erwähnte zuförberft in şinfid)t bes Doc= torz, baj igr furz yor bem 3ujammentreffen mit ism ine פyfers gefallen, fie baber cines andern benōthigt

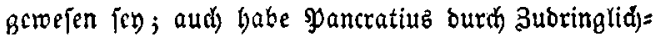

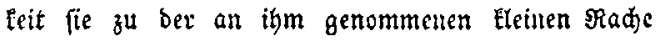
geteig̨t, fömme aber nunmefr, fo igm banad lüfts,

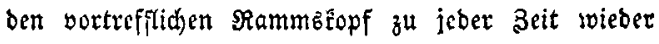

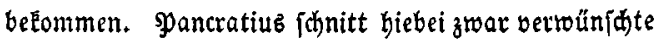
Befiditex, Eonnte jebod ben ibm gemaditen Bortwurf niđjt abläugnen. 
I06

5̧ierauf ging fie zu ihrer \&ebenşgef(fichte über, und wupte mit po viel (Setft unb Befühl zu erzäblen, baß man ifr wenigftens für ben 2 Cugenblide SIauben beimáp.

Rad) ibrer Berfidjerung - unb biejen punlt

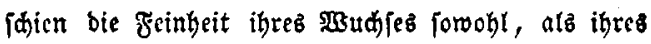
Benebmenz, yollfommen za beftätigen - war fie aus vornebmem (sejd)ledt, unb von ihrer Mutter ouf bas befte exzogen, both aud, alb bie einzige Zoditer, ziemlid) verzärtelt worben. Rady berjelben Zobe war fie fith allein überlaffen geblieben, biz inc Bater fid) zum zwsiten Mable mit einem, für feine Jagre jehr jungen Fräulein, bas niobt im beften Rufe Beftanben, yermählt hatte. Diejer Stiefmutter war fie gleid yon Znjange ber, befonbers aber von ber. Seit an, als fie Gerangetrüht, ein Dorn im 2uge gerefert. Da fie ungefähr in einem after von jiebs zebn Jahren geftanben - jekt wollte fie neunzebn feyn - war Georg, bamals Dfficier, bon fehr guttem baufe unb von ungemeiner Shüngeit, in ihr väters lides Sdjlop einquartirt worben, hatte vor ben $24 u$ gen ber Stiefmutter Gnabe gefunden, feircrjeits ober 
für Raurentia bie beftigfte Reibenidaft gefapt. Sie felbft batte jeine Buneigung erroicbert uno fid), theils burd) bie sirbe zu ithm, theils burd) bie Berfolgune gen ber Stiefmutter, berwegen laffen, bei feinem Wbauge mit bavonzugeben. Hebrigene batte Beorg - bie betheurte fie auf bas Sceiligfte - fid früher ftetz alb ein Ebrenmann und fahr braver Dfficice benommen unb war blos burd) (hled)te (Sejellid)aft, unbejonnente Spiel unb Reigung zum Trunke nad) uno nad babin gerathen, ber Znnführer biejer Sdar zu werber.

Modte an biefer Erzählung fo yiel Waabres fryn, als ba rollte, bab jdjöne Fräulcin wax augemidcins Iid) babei oft fehr, ja bis zu Thtänen berwegt. Mid) ergriff ihre \$̧ehmuth auf rounderbare \$̧eife, unb Eonnte id gleid) baz Mistraucn in mir nidt ganz

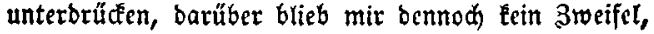
baz Fräulein fen eben to gut ebler Empfindungen,

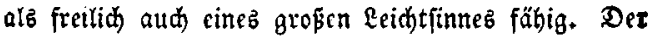
Doctor war aber ober ftelute fidf wenigftenz, bez igm früber geipiclten BetrugB ungeatitet, von ben anges führten umftänben volfeommen überzettgt, unb be= 
IOS

zeigte ber Erzählerin auf eine faft läd)erlichè meife feine wärmfte Thyeilnahme uno Berehrung. Wahrs (d)einlid) um biefen, ifr läftig werbenben şutbigun= gen zu entgehen, madite fie uns auf bie Rothrwens bigkeit oufmertjam, micber nach ber (sejertiflaft zu jeber.

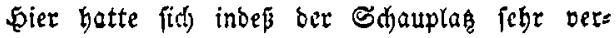
anbert. Die sidjter waren zunt aheil ausgetojht und bab Mujitdjor franb vertaffen; Noten und \$n: ftrumente lagen bort, fo wie im Saale felbft $\mathfrak{F l a}$ la uno Sdyerben in wilber unoronung. Die 3ed)er und anbern Bäfte Gatten fíd gröptentheils entfernt; was nod ba war, fás in einem andern Rebenzimmer am

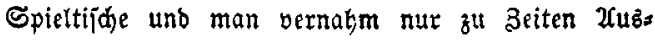
brühe bes 3orns uno Flüd)e. Don Sobriften Gatte man, Bănżlid beraujajt, zis Bette gekradjt, anbere fdnardten auf Stühlen und $\mathfrak{B} a ̈ n$ ten; ber Şameer= baud) פ̧uzziacola rub̆te red)t liebejelig unterm Bifhe, bas sauten = Futteral zärtlid im $2 \mathfrak{r m}$ haltento.

Eaurentien jhien vor biejem Unblicte zu graucn. Sie legte ince şand auf meine Shulter, fah einen Xugenblid ftill zu Boben unb idlug bann mir uno 
hem Dottor vor, ba wir hler nod bie pinzigen $\mathfrak{B}_{i}$ jonnenen soären, bis zu నagezanbrud) beifammen zu bletben.

T3ix gingen wister in tas Rebenzimmer uno \$ans cratius bradte cine Flajthe uno orci Gröjer mit. Eaurentia nabm am offnen zenfter, bab die Xusfidjt

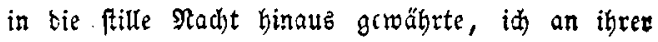
Seite, \$pancratiub in cinem gropen Sorgenftuhle

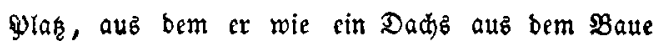
bervorgufte. Dod) wollte, aller gegebenen mübe

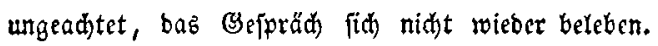

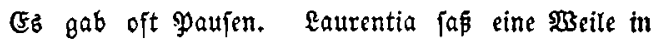
(id) verfents; bann - als wolle fie fid) mit (Scrwalt ber Freute in bie 2rme werfen, hiep fie ben Doctor. Yuzziacala's Raute herbei holen, um uns auti etwas - was abcr nun gleid)? - nun, bas sieb yon ber artigen $\mathfrak{B}$ ogerffellerin, zum $\mathfrak{B e f t e n}$ zu geben.

Sie fang nun in franzöfitider Spradje ein sieb:

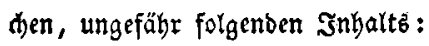

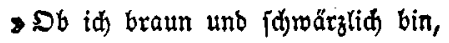
Mid) nidit Sdnee und $\Re$ ojen fdmutecen - 
Franbrer! ift zu jpröb' bein Sinn,

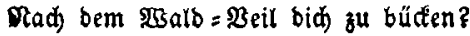

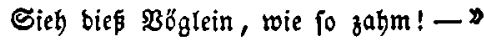

unb bei biejen nedend zum Doctor, ber baburd Bereight idnell uno

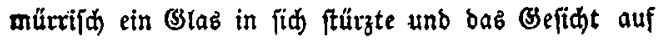
ben Iifid) legte, alb wolle er 佔lafen -

\Sieh Dieß $\mathfrak{B}$ öglein, wie fo załm!

Irauett's wobl, baß̄ eš bură Sd)lingen

um bie goldone Fritigeit kam,

Die voll fdiwarzer $\mathfrak{B e e r l e i n}$ bingen?

Da jeşt Yancratius wirflid entidjlafen fdien, blidte Eaurentia unter ben langen 2 Bimpern beroor freundlich nat mir auf, idlug bann bie Jugen nier ber und fang, ofne fie zu erbeben, bod mit leidter Writerteit, bis zu Ende:

- Sdroarz ift bas Bewanb bet Radt;

Dod erquidt fie jebes $\mathfrak{R} e b e r$.

Sdwazzem 2ug' bat bobe Wadt

Benub und iar Sogn gegeben. 
Rad)t umidattet Riebesluft,

Eiebealuft liebt bunkle Sd)atten,

Sucht, zu ruben Bruft an \$ruft,

Dämmrung monobeglänzter Matten.

Đirt und Şirtin follummern fü

unter buft'gen Rojenhed"en;

Raht Zurora, wirb gewís

Benus felbft die Sđlăfer wecten! *

So wenig eitel und eigenliebig id raar, eb fofien mir in biejen Berjen denned ein verborgener Sinn fu liegen, bet mid) unter bermaligen umftänden jebr in $\mathfrak{B e r}$ jud)ung gefübrt yoben würbe - bätte nidt bießs Riebdyen mit bem meinigen zum sobe ber $\mathfrak{B r a t =}$ netten zufällige 2febntid) keit gebabt! Jal bad)te meic net janften, unidulbigen Pyyllis; th brücte bos Bitb unterm Boller berftohten ans Serb, iprattg

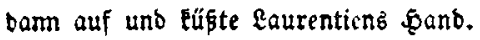

$\ngtr$ Iht fingt jđjön, Fräulein! « - rief id) mit

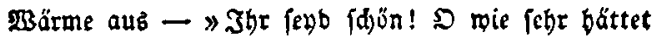
Jłr einen eblen Mann beglüdén tönnen! Wie bebaure iक Eut! ! 
112

Raurentia jhien betroffen. Sie bante mir burd ein Berbeugen bes Sopfs, tänoclte nod ein weinig mit ben Saiten ber \&aute, warf bann baz Inftrtu= ment auf ben alid und entfernte fidi.

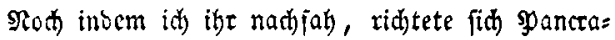

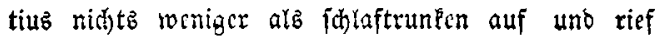
mir leife zu: "Nun, bu bift boh ein $\mathfrak{R a r r}$, wie

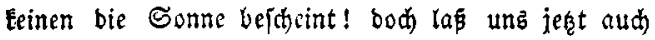
behen - id bin io verorüblic, id bin fo rotb Dod) - scria in crastium ! "*) Sonad) fud)ten mir aud cinen Sdlafwinkel und rafteten einige Stunben, bis unz ber Sonnenjđein weette.

Xla wir erroadt waren, begann Şerr spancratius mid) mit einem $\mathfrak{B e r} \mathfrak{e}$ aub Svibs Amoribns aufzus siegen, und Eonnte burdjaus nidt einfeben, wie in

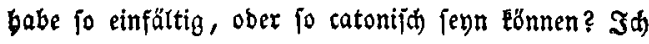
hatte gegen Norgen von \$ybllis geträumt, aud nad) langer 3eit zum exften Male wieber gebetet. Jeşt ging mir bas fecrz auf und ing geftand inm, baß id eine Beliebte babe und nur ber Bebanke an

*) Daz Ernfte auf morgen! 
fie mein Sđutugengel gewejen fey. Er fatrte mid mit ben fleinen ftedtenden 2ugen lange an, und fiel mix, ba id ihm offen ins (sefidht idaute, beftig (d) Eann nidht fagen - um ben 5̧als, weil ex jo weit nid)e langte, lfonbern um ben $\mathfrak{E} e$ ib. „ $\mathfrak{D}$ u bift nidjt nur ein braver $\mathfrak{B u r}\lceil$ de,

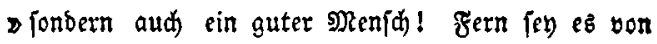
mir, über ctwaz zu fpotten, baz fo jelten auf Erben gefunden wirb! 》

$\searrow$ Uber mit beiner Rlugheit $\ll$ - fubr er bann fort - 》 iftz befto übler beftellt. Darum laß mid für bid, ober viclmebr für unb beibe forgen. श̧冖̧ nidht ber Ealgen und bereinft bie șälle zu befährben, unb - vergält mix bie Deliła meine berbammte $\mathfrak{g}_{i \in}=$

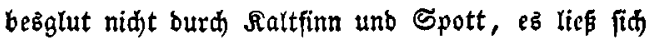

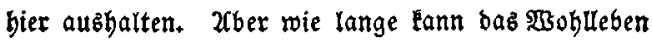
bauern? und bann - 2ajo! - bleibts boffentlid

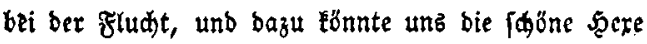
gat wobl begülflit) ienn. *

Er ertheilte mir hierauf nähere Eretärung und iprad: \# Şab' id gleid) bie vollfite urjad)e, bab Dbriften $=$ Fräulein zu baाTen, fo mus id bod gerecht 
II4

fcrn. Mag an ibrem Märdjen fo viel feyn, als ba will, fie bat bas ältertidye Şaub wirtlid aub siebe zu Seorg verlaffen. Nod ift ein Iheil bicjer Riebe nid)t erloid)en; (Seorg trïgt $2($ d) tung vor Raurentien unb fie vermag über ifn, bon Foll ber Iruntenteit aubgenommen, Allez. Zud ift fie zwar leichtfinnig, bod nid)t bözartig; fie füglt zuwciten sieue und mödte biefem berumidreifenden, gottlo[en, wenn aud) äuperlid) glänzenden feben entgchen; fie ift oft zum (S)uten geneigt, fogax gropmüthig. ' baft bu gethan, fo erwärmt bu erift von biejer Sonne fdienft? bu baft fie veriddmäht, gefränkt, beleibigt. Dod) nod), glaub' idh, ifts acit einzutenten. Suhe fie allcin auf, entidurbige beine fyludjtjamkeit - ein red)t artig fdwakender (Sornoon bift ou benn bod)! - fpiegle ibr eine geheime, aber defto feurigere Riebe vor, und gelingt bies, fo thue ithr ben \$orjhlag, fie mit SBeiftand beinez fidus Achates, will [agen, meincr, zu entfübren. Jh) roctte, jie geht barauf eim, unb hilft uns bie ganze lobblithe Mojeljoar über= liften. Sind wir bann aufier Sscfuhr und ou bleiblf

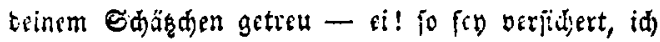


roerbe midi ber armen Berlaffenen aufo traulidjte amehnten! «

Dasి Eeģtere glaubte id) bem Doctur redit getn;

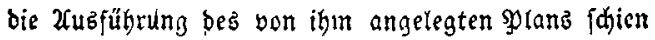

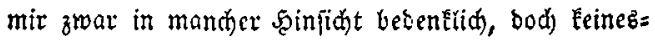
wegs unmöglid,; ber Bebanke, zu entkommen, woar mir fo jüßs und warb mir jest burd zörtiche Erins

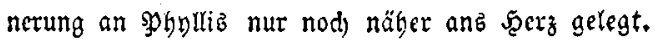

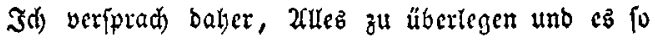
gut, alb id) vermüge, int

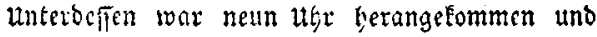
bie nod) geftern bor $\mathfrak{R a a ̆ b}$ auf bon ganzen beutio

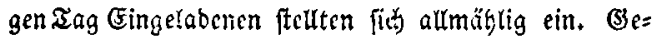
org unb bie übrigen Irunfenbolbe, in ber agat unvet: เwüftitite Sattren, waren fdon wicber auf ben $\$ 8$ eincn. (Ein herrlidyes Frühftü war aufgetragen, und ba augenfdeinlid Die Jerren Stäbter ben Soldaten nid)t trauten, fo yeriprad)en legetere, fowogh auf parol,

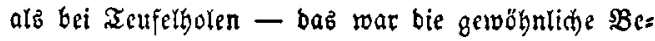
theuerung - das keinem, weber freien Rebenz, not 3utrintens balber, cin seib grideben folle.

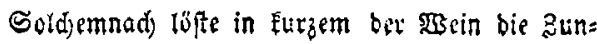


gen, und ber Scauptmann ber Stabt = Befakęung, ein gar bieberer unb verftänbiger 2Crter, fing an zu Ela= gen, baß bie beutiche Freiheit unterbrü art werbe, baß felbft bie freunde ber Deutjhen vor innen gehapt würben, baß nirgende mebr bei bem Artegsivolfe Stern und Seegen fry, wie bod) fonft unterm Sdwe: bentënig, unterm 5erzog Bernjaro, unter bem \$ap= penfeimer uno Spinola!

Dešbalt « - fiel ihm ber vorlaute und ruhm:

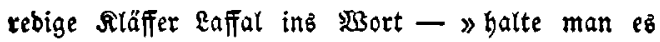

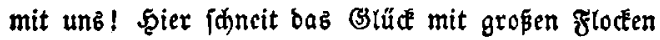
unb all" unire Feinde " - biebei fah er ben Sdult= thei und cinige $\mathfrak{B u ̈ r g e r}$ an - »müfen zu Sdanden werben! *

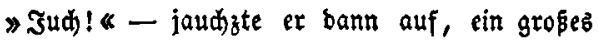
Yaß = Glas jaroingend -

* Dien' id bem Einen, fo Erieg' id) Eein Getb; Dien' id bem Zndern, fo hast mid bie Dien' id zu \$Baffer, jo wägrt mir's zu lang, Dien' idf zat Felbe, was hab' id zum Dank? Juthet ! es lebe ber einzige ţeld, Der unz läpt fteblen, fo viel uns gefällt! : 
Sicrauf ftürzte ex ben 20 ein in Einem 3̆uge binunter uno beid)los :

》 Frifd), uneerzagt, behergt und wader!

Der Ghanfe Săbcl ifi mein \$flug,

uno Beutemadjen ift mein $2\left(d^{\natural e r}\right.$;

Damit gewinn' it) Sold genug! *

\) Freilid! ! - fuhr ber Sdulttheis betaus, ber vielleidft nodh von geftern her ein boppelt freiez 2Bort zu baben meinte - \ab merken 3 ürger unb Baucrn nur allzumohl! «

\Der ift bes Ieufele, ber fid) eines Bärgets

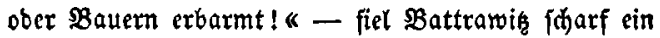
- bat mán nux exft Einen niebergemadt, fo iftz einem boi allen 2unbern, als erichiese man cinen Sund ! *

》Dab merken wir wohl! \& - verfeşte ber ehrs

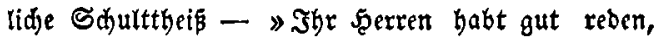
weil ibr wift, bas wir Eud nidts thun bürfen; anjonit -

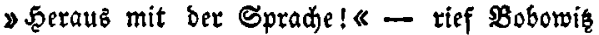
nun aud) lozbredsend - wber ift bes Teufets, bor nidit Jlles jagt, wab er benft! \& 
118

Es begann nun ein Streit und Betümmel, bej= fen zubgang id jebodi nid)t abwarten fonnte, weil mid) ber überall $\mathfrak{Z}$ ugen havende 2 reguz, Freund Syanfratiti, auf bie Seite zog und mir zuflifterte:

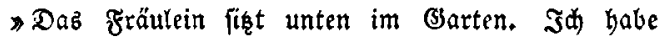
bid) bei ithr gemeldet. Wुär' id) an beimer Stelle num, mad's flug! - Speluncam Dido dux et Trojanus eamdern (teveniumt*) - Proficiat! "

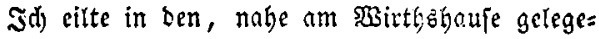
nen, red)t Gübid) arbaltenen (Barten und fand $\mathfrak{L} a$ als rcntiell in einer $\mathfrak{R} a$ ube. Gie war noth im Morgen= Eleibe, jebr fein, both einfach weiß getheibct; bie

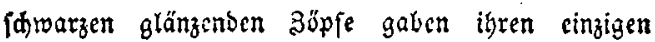
Edjmud ab.

Eaurentia jah tiefinnig zur Frbe und forien gerocint zu haber. Sie crbob, ba ith ihre pand faste, fowwermüthig bie Zugen; thre blaffe getbrid)e Farbe, bie ist faft bas 2rnjehen eineb Steinbilbes lieh, theilte ifr etras recht herzberwegentes mit.

*) In Gine Brotte fommen Dibo und ber Tro= janild)e \$eerfügrer binab. Bctanntlich aus Birgil. 
J゙h füblte wahre Theilnabme, innige Mitleio für

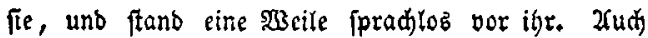
ifr fhienen bie șorte zu feblen.

> Mein Jnntes jagt mir," - begann id baum mit ungegeudettem Befühl - 》 baß Ihr nur bur unerfahrenheit, burdh \&cibenichaft ober burd) unglürt in eine, Eurer nidyt würbige Rage verjeget worben fenb, unb bas Bertrauen, weldjes mix Fure eble Brftalt, Eucr ganzes anmuthiges 2 Befer einflobt, gieft mir ben Muth, dieß zu wieberholen."

Sie jah mid) mit ben gropen feurigen 2lugen prüfenb an. 》 Iht fönnt mich nidht aditen «- jagte fie bann jiemtidi fägl - 》 und felbft meine Freube, in bicfer umgebung Einen - Einen beffern Menjdon gefunben z̆ baben, ferbft bie Reigung, bie mid) bebs halb zu (Eud) Kinzog, muв (Fud) von mir trennen.

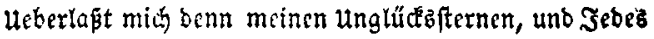
von une crfülle, waz ibm beftimmt warb! «

Raurentia's shlick unb ber Ton ibrer Stinme burdborang mein 5err. Ridt bloz meines şlans eingebene, fonbern audh in ber 2rbjidt, fie zu berubigen, betheucrte id ihr, bas aud id idon burd ben 
erften anblic wurberbar an fie geferfelt worben iey, und baß bie Empfindung, bie fie mir eingeflößt, wahr=

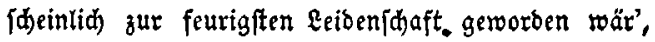
bătte nid)t - meine Xufridtigkeit zloang mid) zu biejem (Seftänoniffe, obwohl es gerwagt feyn modte eine frühere, ztvar nod) verffroiegene, aber unauğs löfdhlide Riebe bieß verbinbert.

\ath liebt? « frug fie haftig unb neugierig zus Gleid, und ferte nach Eurzer şauje janfter hinzu: Shr nennt Eure Riebe eine verjoniegene, und

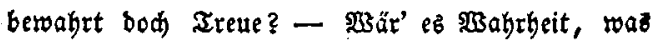
man von euti) Deutfacn riligmt? «

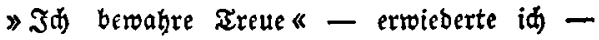
- weil id mir aud son bem Gegenftande meines Eiebe, obglcid) wir unz nie beutlid) extärten, ein (Sleides verfpredien barf. Rur einmal fprad bie folbe, alb id) ihr ein zärtlides, nur leib verrathens bes sied tuberreidt hatte: Şofft bas Befte von bes 3ufunft und einem ftandbaften (S)müth! - aber - in warb immer wärmer, weil Raurentia'b \$Blide

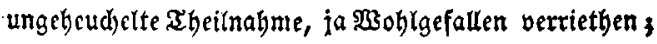
id) rís bas silto meiner \$gyblis bervor - \$aber- 


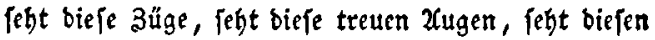
lieblidjen Mund und jagt bann, ob jene 2 orte, bon biefen sippen auzgefproden, nidft ben beiligften Sdinüren Gleid) Eommen? «

Eaurentia betractete bas Bitb. "Euer Măb= d)en $\ll$ - fagte fie bann - \$mú fehr gut fenn uno - ift fehr glüctich! 2(uch) ich war cinft \$cibee, ober glaubte ez bod) zu jeyn. «

Sie erfob sie Zfugen gen Şimmel und verjanE eine

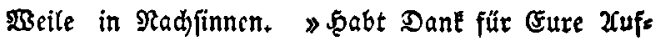
ridtigfeit! « - begann fie bann wieber - 》\$3as Fhr aud bon mir benfen mögt, id werbe (Eures Bertrauens nie unwerth exideinen. Fobert bievon jeben Beweiz, «

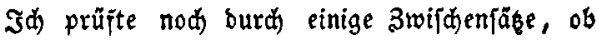
id midf fidjer auf fie verlaffen tónne, und ba id mid) biebon für überzeugt bielt, rief idh fie auf, bie

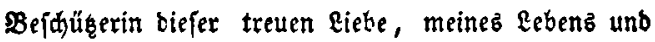

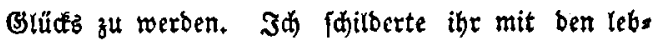
bafteften Farben, wie unglüetich id und mein Freund pankratius uns in unjerer jeşigen aage fühlten; id fekgte binzu, baßj ibre senntniß aller umftänbe, 


\section{2}

ifre 5erzenghüte, und bie MRadt, bie fie über ben Dbriften übe, uns auf bas gewiifiefte befreien tônn: ten; in befdrmor fie bei actem, was ing heilig fen, bei bem 2alter inres viclleidt um fie trauernben Baters, bei ber Wjihe ihrer Mutter, uns zu retten.

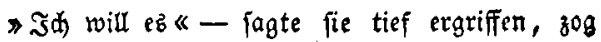

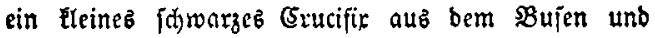
brütte cs an ihre Eippen - 》bei biejem einzigen Undenten einer glüctlititen, fhutbloien Satgend - id)

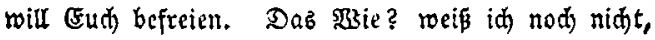
aber id werbe es finben. Georg mus jeben Falls mit ins Bertraucn gezogen werben; aber ex fann nid)t immer, waz cr will. Battraivis und $B_{0}$ bos wik, bribe neiden ism bie sberftelle; fie mistrauen

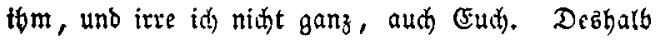
mus zetes idheinbar binter feinem sillten gefd)eben.

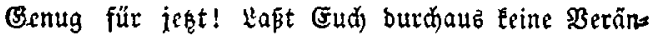
berung Fures Benebmens abmertion, ftellt (Eud) viel= mehr burd̆ bie jeşige luftige Rebensart, ber (Gejellyhaft geneigter gemad)t! - Nad biefen \$\$orten jajob

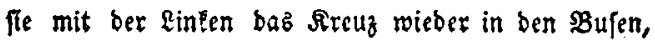


und reidhte mir mit fehr solem, yornchmen 2rnftanbe bic Redte zum Rufie. -

Beftärsten Mutty kehrte id in bie Irinfitube

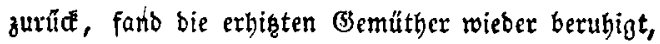
unb rie Bed)brüber, fonberbar genug! zu einem (3es

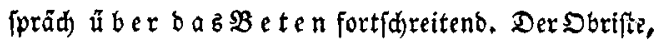
ber nod) aub früherer 2ngevobngeit (tỉ) gern über ernithafte Dinge unterbielt, Gatte nämlid ben Sdhult:

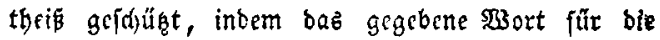
Frcitheit ber Rebe nitht gebrodjen werben bürfe, unb dicfer fobrnn nebft bem Gtabtgatiptmanne es übers nommen, die Gründe anzugeben, wę̆alb bei ben

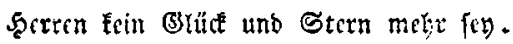

Der Stabthauptmann wirberbolte eben einige Serjolben Eully tid), alb ba war: Mangel an gered)tes Sad)e uno gutem Betvifien - Barnachläifigung bes

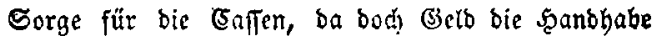

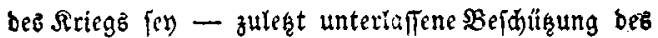

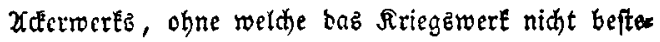
ben tanne - und ging zu eincm neuen aheile feinet Gefegprebiat über. (Er behauptete nämlid), in fral herer 3eit wären bie Solbaten nidt fo lafterhafte 


\section{4}

Drenjaten unb abjdeulide Fluder grwejen. Da babe ę bri bem Xngrifie grheipen: Fort, Brüber, in Botteznamen! Ein jeber fpredie ein Baterunjer! Dente an uniern gnäbigen secrin uno baltet euch reblid)! Nun, Bott bilf! - Dafingegen werte jeşt nur gefreffen, gefoffen, gefpielt und gebubt, uno wenn es ins Ireffen gebe, befomme man nidts zu bören, alb: 2uf, ibr Buridje, baj eud der Donner exץhlag! brauf ins Teufels Ramen! - und waz bergleid)en juredrlidje Morgen =, unb 2(benbjeegen mebr wären.

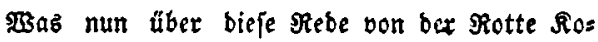
rah für Gefpött und \&äfterung getrieben roaro, will id) gern berfdreigen. Waahr[dsinlid fah man mir etwas von meinem unwillen an. Saffar rief mir böbs:

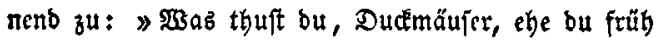
aufftebeft?

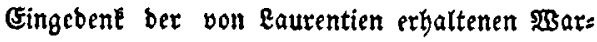
nung, burft' id ifim nidjt antworten, wie id in

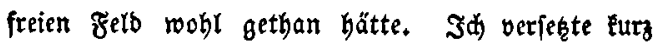

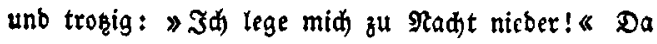
entftanb ein Ģeläd)ter, uno \$Bobonic tief: \aft 
ifn! wern er noh eine Socile unter uns ift, wirb ex gut! *

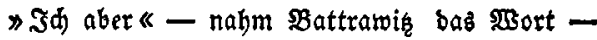
2halte $k b$ bamit wie $\mathfrak{p} a \mathfrak{r a}$, mein liebwerthefter Serr Bater. 2Ura id) zur \$elt gefommen war, gab's gar grope Şerrlibletit und main Bater Eonnte nidt mit fid) eins werben, wo er einen gnug(am mäd)ti= gen und gropen Sanjen zum \$athen finden folfe?

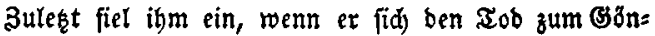
ner mache, werbe er erwig auf Erben leben. Dezs halb - wie benn bez simmels und feiner werthen 2rngebörigen meine ganze Freundiłaft überhaupt nie fonberlid geadtet - bat er ben Der Snodjenmann, ber etwas von ber lifit merte,

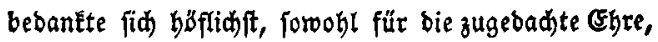
alb für bie 2(nerkennung feiner Madt, nad) welder Zales, vom erften Obemzuge an, ihm angeböre, und fügte finzu, baj er bem wettfen Bevatterzmanne in 2ruem zu Dienft Yeyn wolle, ein Crinzigez, näm= (ii) bie unfterblidkeit ausgenommen; biefe Eönne teinem vom şeibe Bebohrnen verwilligt werben.

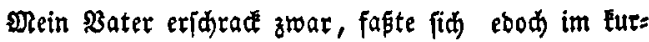




\section{6}

fem und verjeg̨te als cin gar jollaucr Sthelm: $\$$ Ja, lieber Seerr (Gevatter Iod, bej beideibe id mid; aber eine kleine Frift lünntet igh mir boch zufidern, bevor ifr midh abjenfet! " Der adod, ber fids fonft fclbft vom Ieufel nid)t ketrügen läpt, veriprad bas. unbebenflid,. \Die Frift, gnäbigcr 5eert (3evat= ter! 《 - jagte aun mein $\mathfrak{B a t e r}$ - 》 if bie, baz ihr mid) nidst eher holt, bib ids zlbor ein paternoffer

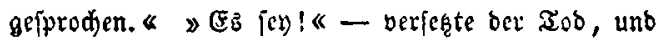

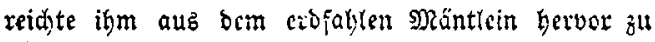

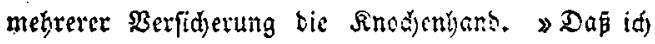
nun Eein Narx wât « - fprad ladjend mein Saier - jemala ein spaternofter zu beten! « Nun mögt

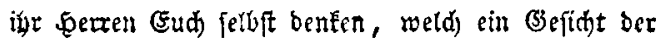
Eerr Tob gejdinitten. Ded) glaube id) fider, Bater Darra lebt nod) zur Ctunde, cz müste ihm dean

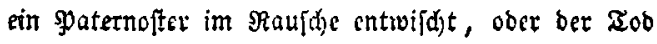
zum Gunbbjott mordeat jeyn! «-

Xuf biefe $23 e i f e$, nur zum Theil nod lăfterlidjer unt rud)lojes, bauerte bas (scipräd) eine feine \$Beile,

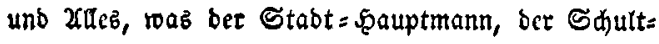

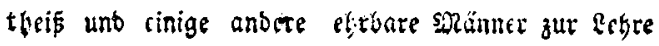


unb \$ुarnung vorbradjten, Diente nur bazu, bas

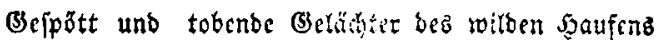
3u mebren. Sh modte, fo weit bies bie filugheit erraubte, bas Mrifte bavon gar nitit mit anbsosen, gab unter ber מand bem Doctor von bem Erfolg

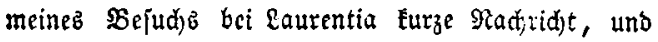
war froh, alz endich bie 5̧örnet zutr $\mathfrak{x} a$ fel riefen. 2ud vernahm id mit inniglidyer freude, bas bas

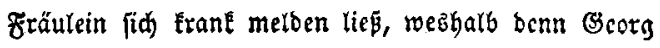
alabald ben Dortor an fie aborbnete. Daś biefcr bie Bielegenteit benusen werbe, bas angefungene WBerE weiter zu fordern und iigeno cinen şran mit thr zu verabreden, Eonnt' ih ohne mzeitercb vor aubję̧en.

Dießmal war bie (Bejelliojaft wod biel gemijater unb bunter. Denn, auker ben fogenannten Dfficieses. Damen unb Sdyweftern, gab es audh andere, bie um Gelb feib find. Nidft ofne bas gröstc \$ebautern (ah) id) mitter unter biejen bie నodter eines Rath8s bern, bic, id weiß nidft, ouf was bern in bie Sände geratyer roar. Reben biejer batte Bobomis frinen plas genommen, unb objhon bie 


\section{8}

2rrme fid Eaum ber bellen Sähren enthalten lonnte, muften bod ber Stabthauptmann und einige anbere ebrbare Männer ibr ganzes $2 \mathfrak{n}$ fehen aufbieten unb felbit ben Sbriften zu șülfe nebmen, um fie vor bem

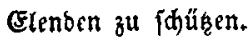

Da bierauf bei నijdye in Eurzem bas Sberfte zu unterft ging, gelang es mix unb einigen 20 ndern, bie Ratbeberrntodyter Geimlid entfichen zu lafien. 2ulein, laum war Boborwiés siés geroabr worden, als ex

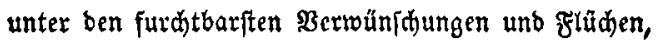
bemjenigen, ber ibm biefen Streid) gefpielt, ben Fod rrobte. Er benahm fid babei ganz roie ein Rajenber, fo bap - zumal ba ein Servitter im $2 \mathfrak{n}=$ zuge war - jelbit viele ber $\Re$ äluber fid bavor ent= fę̧ten unb aus Furd̆t, mit ihm exid)lagen ober von ber Erbe verfolungen' zu werben, ihn mit Bewalt

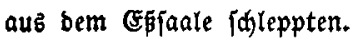

2イł $\mathfrak{B l i}$ unઠ Donnex vorüber gezogen, begann ber Rärm unb baz 3echen von neucm, unb bamit bie \&uft volffommen werbe, hatte Suzziacala alle (Sriger unb $\mathfrak{p f e i f e r , ~ b i e ~ i n ~ b e r ~ \Re a ̈ h e ~ z u ~ e r l a n g e n ~}$

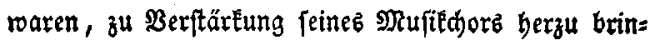


ben Iaffen. Es kegann ein Tanz, wobci benn ber

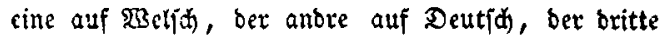

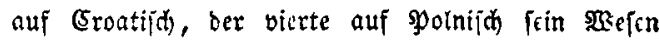
trieb und gewöhnlid ber 2ubtretende dem 3̧unäitjt: ftehensen zurief: 》Dor ift bes నeufelz, ber nidjt mit madht! " Das $\mathfrak{L} a d j e n$ und autrinfen, bas Singen uns בunzin bantrte bis lange nach) Mittermadt, okffon bis meiften (s) äte fid früger vertoren hatten.

$\mathfrak{X m}_{\mathrm{m}}$ folgenden g)lorgen rax mündlid) bie vers traute Funbihaft cingegangen, dap mit geringer

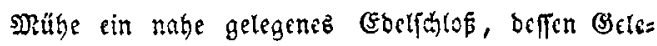

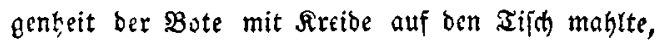
uีberrumpelt unb geplünbert, unb grope Bcute gemad)t merben fönne. Nun war zivar aukgemaht worben, Daß vor $\mathfrak{B e g i n n}$ neuer Sceldenthaten erft bie ganze Ddjenfeerbe burd bie Burgel gejagt werben folle. Da jeboch bei ben $\mathfrak{R e i f t e n ~ b u r d ) ~ b a s ~ i m m e r r a ̈ h r e n s e ~}$ Sălemmen - benn fihon an biefem Dlorgen war Branntwein und Iabad wieber in tebermads genoj= fen worben - ber Muth und bie Bermeffengeit gewadifen twar, 2nbere aber aud nur eime Barre= 
I30

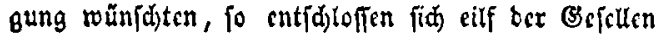
su bcm Ritte.

Die nod) unbártigiten barunter hatten fíd, che fie an bas Sdloß betommen, die Stoppeln vollends abnehmen lafjen unb waren in SWeibskleibern, bod) bie syiftolen unter bem fürtud, bie übrigen als Bauern mit rinem Fäpdon in bas Sdbioß einge= fdliden; nod anbere batten fid) in ber Năbe ver= ftect, um auf ein gegebenes Signal zu Şlilfe zu eilen. Die $\mathfrak{E}$ ift war bollommen getungen. Man Gatte bie beften Sadjen in Bett= Snbelte gepadt, aus welden man bie febern auf ben Sof gefdüttet, unb fünf 9 ferbe bamit belaben. Ein \$̧ofenecht, ber ben Berräther abgegeben, war fofort z̆ ber Banbe getreten, unb eine gar rüftige Mago bes Sdlofver: walters, nad jëmmerliăen Sdreien und 2 beinen, bem fnehte gegen bas Berfpreden, bas er fie fir: hen wolle, gefolgt. Dab Mirdhen ber Solbaten beftanb übrigens bamals blos barin, baß ber $\mathfrak{B r a ̆ u =}$ tigam bie Braut bis an bie Nirdhthür und bann wie= ber fort fübrte; nun galt eine fold) feujde Su= fanna für ein angetrauetes șbeib. In berjelben 


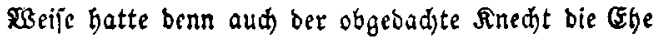

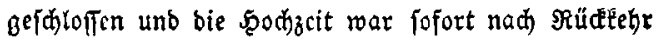
ber Räuber in Saus uno Braus begangen worben.

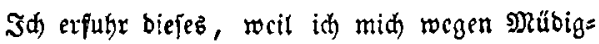
eeit frübzeitig niebrrgelegt, erft am folgenden Mor= ben, ba einige in hellem şaufen midh aufroedten und mir ben $\mathfrak{B r a} \mathfrak{a t}$ wein, wie fie es nanten, vore sett braditen. (Etlidje Rage jaßien bie löblie hen Gejellen roieber ftill unt fteif, bod alfo, als ob fie blob zum Saufen auf Erben wären.

W3eit ubbler, alb ber eben gemeldete, lief ein anbrer $\Re i t t a b$, za bem einige $\mathfrak{Z} a g e$ fpäter funfzegn Freimillige bură bie Botfdaft, bas abermals ein gar fetter $\mathfrak{B i c h}=$ Transport in ber Rähe vorbeiziege, fid) verleiten ließsen. Diefe hatten bie șeerbe bereits erbeutet gebabt, jebod, fid völlig in Sidjerheit glaubenb, obne bie fonft geroognte Borfitht in einem

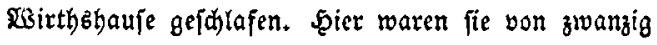
Musfetirern bei Rad)tzcit überfallen unb auf einmal burd) athür unb F̧enfter mit voller lojung begrüвt worben. Die fäänblitgfte frittht war hievon bie Golge gewejen. SRan batte fämmtlide פferbe bis 
auf 3woi im Stidje und, auper einigen Betyiebenen, fünf anbere ber Benoffen in Befangem jaft gurüctaften

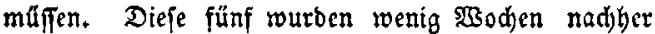
in D. get, 2 frt.

Man verbeimlidite bie Radidid von biefer Słlappe, fo gut eb geben rollte, glaubte aber auf ber anorrn Seite nur befto megr (Bileidgültigkeit, uebermuth und בroţ zeigen zu mü Ten. Deहhalb wurbe alfs neue eine prädtige (Safterei bejdloffen, unb man trieb bie unverfäämthcit fo weit, fogar Den Sberpfarrex und Diaconuz einzlitaben.

Der Mfarrherr war ein Nann von hohen Baben und groper Stanbhaftigfeit, welde er aud), obroohl felbft oft in ber orüdenditen Noth lebend, bei Ge= fabren ber Stabt jattjam bewährt hatte. So bejaß er benn aud beim (Fintritt in biefe Gejerrjhaft, too ex fich freilid als ben getreuen Sitrten mitten unter Wölfen betradhten muste, ben \$uth zu ber Xeufe= rung: Birlyeidt füge es ber barmberzige (Jott, baß er einem ber Seerren nod) bienen Ënne. Diefe \$3orte verleiteten freilich Einige Der (Benoffenjdaft zu ben abjheulidjften Räfterungen; anbere (d)ienen jcbod nad) 
benflidi zu werben, uno id meinerfeits bratute bem eftrwürbigen (Breife heimlid zroei Dublonen für bie Urmen in bie Sand, mit bar sitte, für mid zu brter.

Botomik, ber bick bemerkt hatte, fithalt mid beshalb auf bab unglimpflidfte uno mit frinem Rieb=

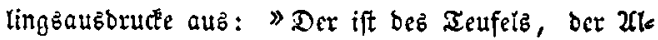

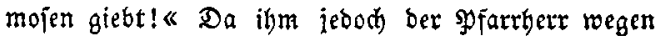
bicjer gottlojen $\Re$ coe zwar väterlidi), bod aud mit bem männlid)en 3ufake ftrafte: 》গুßer nur barum in Nen Siteg zieht, um zeittideb, ungeredtes (5)th zu erwerben; wer aus Ђূabgier ben Frieben hapt uns Den Nrieg will, ber tritt wahthaft in bie Fuptapfen bes בeufers! « raunte er mir inb Dhr: 》ber Pfaff foll mir bas Wzort fidter bezaglen! «

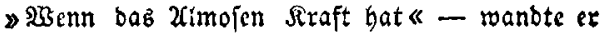

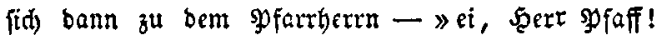
fo lupt mir alth etwas (seifftides zutommen, näms lich, bas id feft werbe roiber Scauen, Steden unb Sd)tesen. Dann jollt $\mathfrak{S}$ hr aud von mir eine Spende exhalten. «

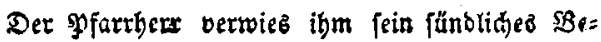


get)r mit 2Cnführung beb 3auberer Simon, bir aud) heilige Dinge um (B)lb begehrt, unb fuchte ihn 3u belebren, baj nur ber Soldat feft fen, ber ein gutes Bervifien habe unb auf ben Seern ber Seer: idaren vertraule. $(\mathbb{E r}$ verbolmetid) unb erflärte iĞm baz Sprüdtein: Si tibi Christus adest, fiet tibi aranca murus. ")

Bobowis ftellte fid aufmertfam rerbenb und

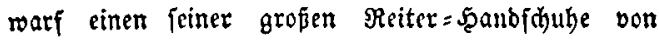

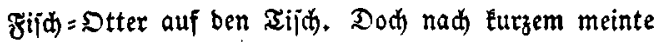
ex, bea פfarrers פrebigt fen ihm bei reitem zu bod, nagm ben sanbiduly wicber und ftand, mir einen gesinl gebend, auf. $\mathfrak{D a}$ in ihin folgte, zeigte er mis mit $\mathfrak{E}$ ad)en ein Eleines, f(tberbejdlageneb Bebet= bud, bas ber Beiftliche mitgebradjt und nebft bem griefterhute auf ben Tijath gelegt hatte.

Db nun wohl ber פyfarrberr feinen $\mathfrak{B e r l u f t}$ in furzem bemert batte, traute ex fich bod nidt mit ciner $\mathfrak{B e}$ idulbigung berbor, fondern beurlaubte fith

*) Jłt Eụriftus mit bir, Eann bir ein Spinngeweb jur Mauer werben. 
blos mit feinem 2umtb̧bruber, unter bem Borgeben, baj fie einen Iranken befuchen müipten.

Nir war ibre Entfernung fehr angenehm, reil fie baburh/gewiß mebrern Rränfungen uno Beleis

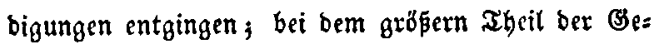
fellid)aft fano ein (Sleiches ftatt, weil bie Meijten gegen alle (Briftidice eine 2 rt Sd)eu trugen, unb fogar äußerten, fie fönnten feinen feben, obne an bie Balgenleiter zu benten.

Man lick nummebr ber wilbeften Euft ben sügel

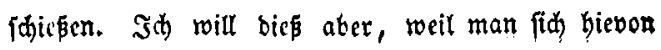
fbon aus bem vorber (Erzählten einen 3 egriff maden funn, mit Stillijworigen übergeben unb nur eineह,

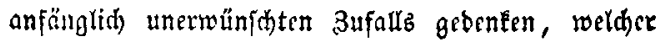
gleidhwohl bem, bereitz zwijhen Eaurentia, bie nod immer sie Nranke jpiclte, bem Doctor unb mir vers abrebeten slane geviffermajen bie Sans reidte.

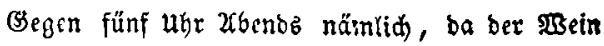
faft eben jo reid)lid über bie sifche, als in bie (5urgeln frömte, fercte fich spancratius, ber eben von Eaurenticn kam, in ber Näbe cinez Shentijळes

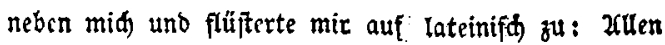


186

umftănoen nad Gabe bas Brciten ber SRojelfdar an ticjem Srte die längfte 3eit getauert; ez fheine, als fange mon feit bem lekgten unglüde an, ein wonig bom Ieufel zu träumen; Raurentia rolle mich morgen fith nod einmal fpredien und bebhaly unter bent Bormanbe, ber Gonnenwärme zu geniépen, wieter in ben Garten geben.

J4) weis nidit, ob bas Latrinif(t)resen ben $\mathfrak{B} 0$ bowis und Iaffal vetbrop, ob bicjer vielleidit jenent meinen 2Untheil an bcm Entfommen ber Rathsberrn= todter verrathen batte, obcr wab beibe fonft var= modite, fo an mid- zu bringen, geuug! fie liépen ein

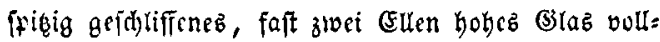

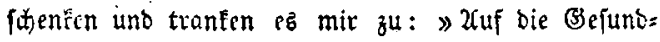
beit bes frömmiten Solbaten, bcr bie mciftrn Rulbe

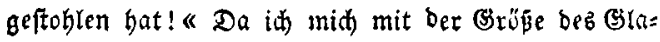
feb entiduldigte, rief Bobomigs: ber ift bes IeL= fels, ber nidht fäuft!

um Streit zu verbüten, fagte iă, bas id̆ bes Guten bercitz zu viel gethan; bod rolle id, um ibm gercht zu retoen, einen Iropfen trinken. (E) 


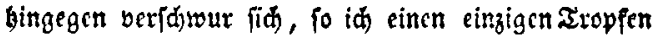
veridultte, fen id bez Tobes!

Jekst foljwoll bem Doctor ber Fanm. Bor Burn glüheno rief er mir zll: , hic ne scurra tibi nortem!" ") bem Sobowis aber: 》 Meint ihr, wir fino nidt Nannz genug, ef mit cud) aufzunegmen?

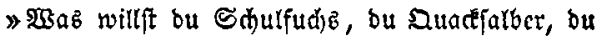
SBladbogel, ber erft jeçt bei ber Miferlăar ein wenig zut Febern getuminen? " - fagte Raffal verärottid * mach' nur fetivf beine Esurgel fertig, zu jaufen!e

Diich verbrof ber 5̧odmuth ber beiben Buben und id) fprifte bas (Blaz in hohem Begen gegen Decte unt Manb. Dief war faum geithehen, als

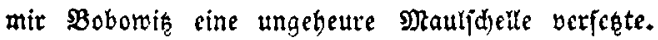

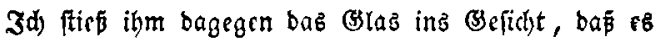
zerid)metterte und ifm baz Biut heruntertief, mir

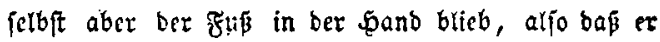

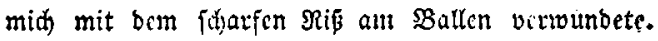
Man fiebet bie Rarbe bayon nod). Dann warf id

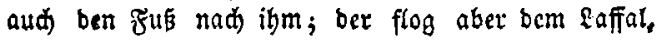

*) Diejer piaferbăting will bir ben Zod brohen? 
138

ber zwijhen uns jprang, an bas Şienbein, baß ex binte und blutete.

İ) unb ber Doctor ftanben nun für cinen Mann unb ficher woär bas Sd)armükel febr ernfthaft worben, bătten unę niđ̆t Battraroik uno anbere von einanber geriffen. SBobowis vermas fich bod), daj er aud mein solut feben müfTe, uno fallz id ein reblider Serl feb, wollten wir morgen vor ber skablzeit die Sadje auf gut folbatifa) autsmaden. Daffelbe ver= langte \&affal unter vielen Drohungen und (Sropfpre= Gereien bom Doctor, unb fo gern wir beibe in Fries ben geblieben wären, wir musten, wollen wir nidt ofter genedt werben, bie 2usfoberung annebmen. शBir gaben unb alle vier barauf bie bănbe, brahten unb jobann (S)läfer zu unb thater gegen feitig Beitheib.

Sierauf giengen wir, um unz follafen zu legen.

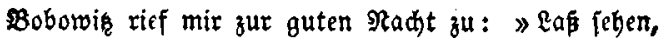

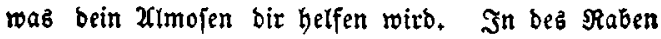
Magen treffen wir wieber zulammen. " $a f f a l$ hins gegen gab bem Pancratius nodmals bie Spant, füpte babei - wab unb gar läherlich dưnete - ein roth. taftnes Banb, baz um feinen ફ̧ut geênüpft war, unb 
ricf: $\gg$ Sdhlaf roohl, Doctor, unb befichl bid) (Sott,

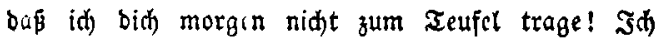
aber befehle mith jegt und immerbar in meiner $\mathfrak{R}_{\text {ieb }}=$ ften Seurb unt (3nabe, uno werbe bid) burd) berielben Bunft morgen fdlafen legen, «

Mir war bei allebem ber angefponnene Şanset fehr ungelegen. Der Doctor hingegen verwies mir eb, als wir unb in unferm fïmmerlein befprad)en, bebauptete, baj toer bie 3unge als Sdjrert braudje, nidt eben zu fürthten for, und ermahnte mich, nue

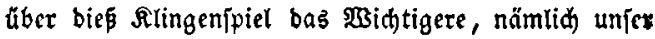
Borgaben, und zufderberft ben Bejuch bit bem Frätw Lein, nid)t auper $\mathfrak{Z} d$ ht z̆u laffen.

Sonad) laufate id fait mit anbrectenber Mozs genröthe am Fenfter, fah gegen aht ubr Raurentien, ihrem Beripredien gemäp, bem Barten zueilen uno folgte ihr nad) Eurzer Frift.

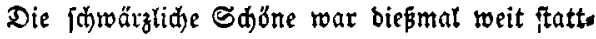
lider, als bei ber exften 3ufammentunft, gekleiber, unb trug logar eine Jumelenidnur in ben socten. (Es büntte mir, als fudje fie fid ftolzer und Eecter, ia fröblid utb freubig zu ftellen - was jebob) nidt 


\section{0}

Kange aub̆bielt. Sie gab mir nad ber erfren $\mathfrak{b}_{e}$ grüfung zuförberft ifre Bejorgnißs wegen bes beves: ftegenben Doppelten 3reifampfa zl ertennen; bod babe :fie ben Dbriften vermorlyt, bas er uno mebrere ber (Bejelfichaft zu Bermetbung jeber Şinterlift gegen= wãrtig feyn würben. Dann tam fie auf unfere $\mathfrak{B} e=$ freiung, fragte, ob wir jattjan mit (3) blo verjeben wärcn, unb ba id bicfeb bejacte, frgte fic: \$ Eo bleibt mir nidts übrig, alb eut) bieje beiben sperga= ment = Stüąe einzubänbigen, Georg hat fie mir für euch) gegeben. Tan gebraucht fie arz zimulete, unb hălt fie für Ialiemane, bie gegen jeben Feino unb Berfolger unfidtbar mad)en. Dićp aber an feinen Ort geftellt, fo werben fie cudj zum beften Freipn bienen, falla ihr auf senoffen ber Mojelínar ftoset. Denn alle Eennen biefes zeiden, objhon nur ble Bornehmiten unb Buveriäliigften es befiten. Beigt tar es benjenizen, bie eud viellsid)t auffangen, fo wiro man in cud) alzbals vertraute hodiwiditige Boter erbliden, und eud fogleid) zum bebülflich jeตn. «

* unb nun $\ll$ - fegte fie betlommen binzu - 


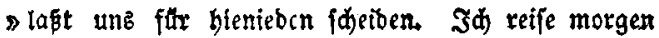
oon hier $a b$, und bie Sdar wirb aud nidt lange mehr raften. Eebt mohl! (Srǘpt von mix eureßraut, unb - fie folle zumeilen für eure Befreierin beten!

\$3ir waren beibe tiefberoegt. Shi küpte igr bank bar bie Jeand und riß mid bann geroalt

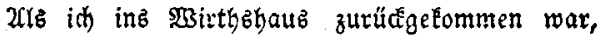
fand id bercitz bic ftreitenden Iheile unb biejenigen, welche ąeugen bes (Sefechts fenn wollten, in bes Briniftube verjammelt. Sobowis und Eaffal joitenen nur beabart geruht zu baben, un nunmebr ihre Buns

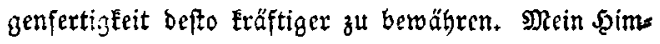
mel! wab prahytton fie - wie fie mit uns umgeben wollten! roie fie im Nirieg auferzogen wären, wie vielen fie bas Ridjt ausgebrofen, wie mandjem $\mathfrak{p}$ faffen und $\mathfrak{L} a n d z$ Enedte fie bas Garaub gemadht Gätten!

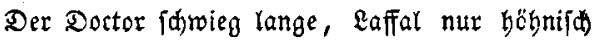
anjegend. Enblit) ris igm ber Bebulbefaben. 》) Sdroeig endidi, (Bropmaul! « - rief er ihm boge haft z̆ - 》was gilts, id) babe für bid cinen Stop, ben bu mir nidht aubjaläglt? roas gilts, id ipiefie

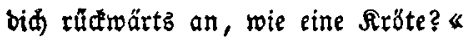


142

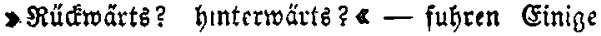
ber Reuter auf - 》bas follft bu uns bleiben lajfen! Das wär ein häßlid)r, unehrlid)er Stop! \&

\) $\$$ fagt mir, wenn Bortheil unb Bortheil gleid), (Se: fidf gegen Befidit ift, nennt igr bann ben אampf unehrlid)? \&

* Bortheil gleid), (Befid)t gegen (Sefidt meinten jene - vwer mag ba wab bagegen baben? \&

- Nun jo gebt $\mathcal{W}$ dit, ibr tapfern 5eeren! * -

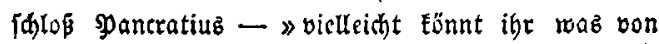
mir lernen. Seşt fommt endlid und laß̣t uns zur Sadje jareiten! *

Goldemna berf vügten wir unż fämmtlid bots

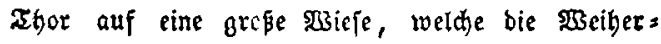
ERatte genannt warb. unjere Bregner waten bes

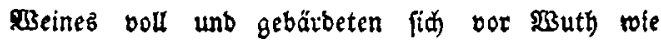
unfinnig.

Zfts wir angelangt, $30 g$ id nid)t faut vom Reber.

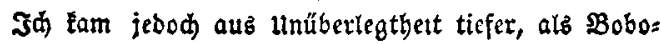
wie, ju fteben, fo baj biejer, um einen ganjen Sdub böber ftefend, bebeutend im Bortbeil gegen 
mid) toar. Sasir forticn eine 3eit lang mit rodbjelns bem Blük, unb licfen zulest grgen einanoer, baß beibe fharfe Rappiere neben ben Ecibern bingingen.

Runmehr warf Bobowig ben Degen weg, ume griff mid in ber Mitten, riß midh zu Boben unb ftieß mir mit ben Sinieen gegen bab Serz, als wolle ex mid rabebreden. $\mathfrak{I}$ d) abcr batte meinen Degen nod) feit in ber frauft, uns fdhb unb bämmerte ibm mit bem Rreuze beffelben fo lang auf ben Ropf, bis bas Blut wie ein Sptingquell beraub fprüste.

Fr ricf: "Das ift toie ein Mörber gehandelt! *

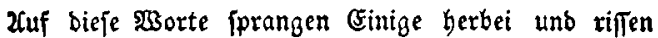

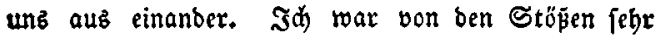
Lbel zugeridjet und empfand ein fharfes Steden auf ber SBruft, reldes erft lange 3eit nad)her burd) warmes Berftenwaffre und eine grüne Salbe, von Yancratius mir aufgejdrieben, fid gänzlid berlorsn bat. Dod aud Boborois batt' es nidut umionft.

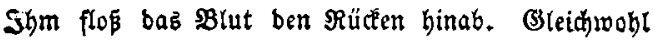
musten wir auf 3ureben ber Benofien einander bie 5anb geben unb unb aljo bertragen.

Nunmebr mathten fid bet Doctor uns Raffal 


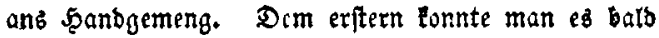
abmerlen, bap ex nidst bas erite Mal bei betgleithen fan; benn er fprang Gerum, wie eime 2kelfter, barb auf bicie, balb auf jene Seite, fo onj ber etraas

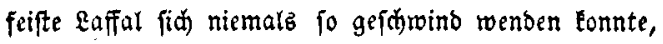
inm voll gegenüber zu ftehen. Endidi erjah er fid feinen Bortheil, lief auf Laffal ein, fprang bunn fdnell binter ibn, kehrte bab æappier mit beiben

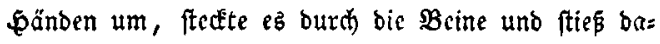
mit $\mathfrak{L}$ affal in bab 3 cidffte, jo baf biejer, ehe at bas Bejdethene redit inne worben war, zlt Boben lag.

Die Meiften erhoben ein lauteる (Selähter; einige gaben bem Doctor unredht, mehrere Redt). Eaffal hatte feinen Dold herausgeriften, als wolle er auf ben Doctor zu. Ere must' es aber rohl laffen, ba cr nidt aufaufteben vermod)te, fonbern in bie Stabt getragen werben muste. stsir übrigen gingen zum Ej̃en und biemit war alleb abgethan unb vergeffen.

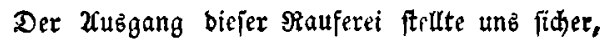
das fid) fo bald Niemand wieder an unz reiben werbe, war uns aber auch in anberer ড̦infidft fche willeom= 
men, weil er, als wẩ er verabrebet, in unjern fđon lăngft entworfenen \$glan papte.

Sancratius erinnerte mid bep bei ber aafer, inbem er auffpringend aubrief: " Ih mus ben armen gefpiesten Frofd dod) ein roenig famieren und bc= pflaftern! « und, noth fain B̧taz leerend, mir zu= trank: , Mesuento mori! *)

Saum war er fort, alz id) ftitl ward, ben אopf in bie Sand legte, und auf $\mathfrak{B}$ efragen unter Stöhnen uns Seufzen gerwaltig über Bruftidymerzen flagte.

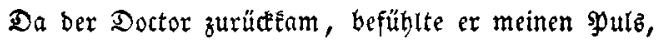

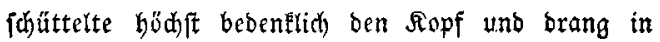
mid, eilibit baz $\mathfrak{B e t t}$ zu juchen.

(Ex behandelte mich son nun an alb einen höhjts

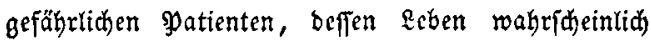
Durd) einen Blutfturz enden werbe, befudte zmar von 3eit za 3eit audi \&affal, blieb aber jowohl ben 2cbend über, alb aud), wie ohnebiés gefdah, bez গadjtz bei mir.

*) Srie ez hier genommen: „Bebent, bas bu trant werben, wobl gar fterben joluft!" 


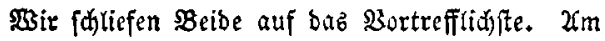
Drorgen aber, in aller Frǘye, beftrid mir ber Dot

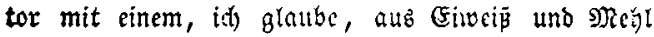
gemijatem Săbblein, Das ganje (Befid)t, fo bas id), alb er mir ben Spieget borhielt, jubier vor mir jelbja exfdrade. Sobann that ex über mein und $\mathfrak{E} a$ fralb Befinben bem Drriften Melbung und Fegte hinzu, baj

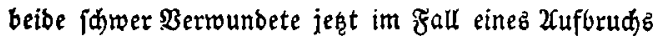
unmöglid forgen fönnter.

Der Dbrift Beorg Eam in Eurzem, alz roolle ex (ith) mit eignen Uugen überzelugen, an mein $\$ 8 e t t$, wo (id) es benn an $\mathfrak{B i n j e l n}$ und Stöhnen nidjt fehlen

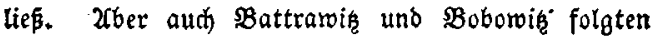
itm auf bem Fufe. Sach langem Streiten, roobei biefer beiben $\mathfrak{B e r b a d}$ gegen mid unb ben Doctor (iid) mehr als gentig ăuperte, indem fie fogar unfer Rateinijhreben anfübrten und für eine $2 \mathfrak{x t}$ Inodjen= jpergifd (b. h. Diebbiprade) auzgaben, warb endlid

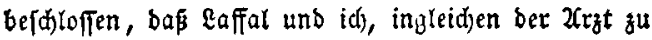
unjarer Berpflegung, zroar zurüclebleiben mödten, jebod) zut umerer Bebertung - fie meinten bamit

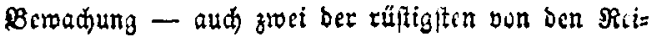




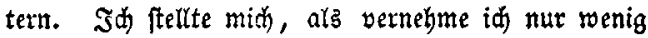
unt fönne nod) weniger ipred)en. Der Doctor aber beftand, obrodhl nur zum Sdheine, zu unjerer eigenen Sid)erbeit febr eifrig auf einer zurücţzulaffenden Batat.

Um $\mathfrak{m a d m i t t a g e ~ b i e j e z ~} \mathfrak{T a g e s}$ zogen, wie id

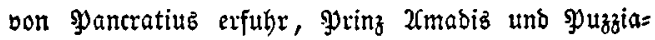
cala mit ifrem (Sefolge ab; gegen Mitternad)t aber brach die ganze übrige Banbe auf, bod wie herge= bradt, mit bem \$orgeben, dap fie in wenig Tagen

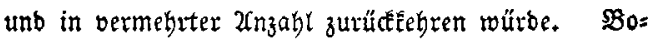
bowió hatte zwei ber ftäreffen, roheften, und bob: hafteften $\mathfrak{B u b e n}$ auşgejưt, mit bem gebeimen $\mathfrak{B}_{e}$ fegrl, uns frreng im Xuge zu behalten und, wenn id genejen, nadzubringen.

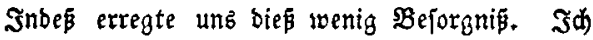
ftelte midh am näbjiten Murgen, fo virl möglid, nod) binfälligex. Den Eaffal yatte ber Doctor megr

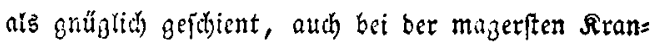

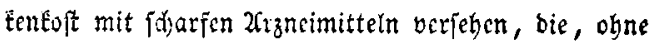

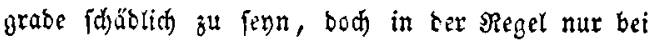

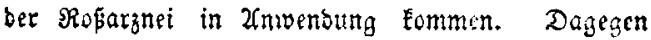




\section{8}

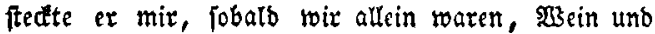
bie nahrhafteften Lefferbiblein zu, fo daj id), obrobl

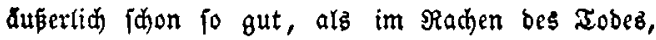
mid) inzgeheim trefflid wohtbefand.

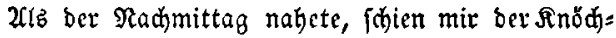

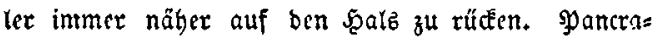
tius rief bie Reiter berbei, um inbep bei mir zu blcis ben, maajen er in bie Stabt =2rpothefe wothe, um nod) bab Rescte zu verrudhen.

UTts ex zurüdgetommen war unb mir einige Löffet

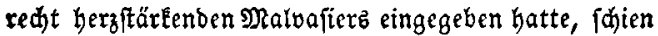
er immer beforgter zu werben, fügrte mir alle 2 Uu= genblite nach bem פulje, rieb mir bie Sđłläfe uno

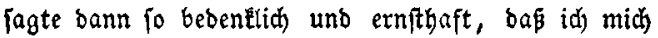
faum bes 2 (ufladhens entyalten Eonnte, zu ben zroci

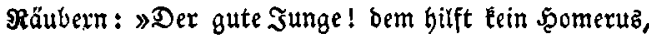
nod) Zriftotelez, kein (Shlyftiriluz, not) Laxantius, lein Bombaftuz, nod Syancratiub mehr." Sh that, als bätt' id) etraa von ber 2fubftellung biefez Reifes paffes verftanden, fah ben Doctor webmütrjig an,

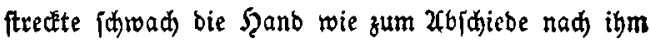

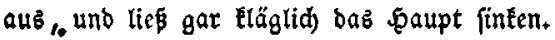


\ In ciner harben Stunbe ift ex Ealt》 - begama Dee Doctor betrübt von Reuem - 》aber lapt uns alz chrlidfe (5ameraben bei inm auzhalten! $\mathfrak{B}$ ir haben noch Şseinz bie Züulle; babei läpt cz fid allens falle sis in bie Rad)t wathen, 》

Die Wähter fanten bieß jehr vernünftig gefpros den; Brope stseinennnen wurben herbeigefdaft und Der Dottor, in bergleid)en Sunftitüretein ungemein

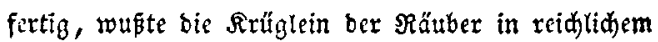
Dlaaje mit bem -Sdlaftrunke zu fülten, ben ex fï fo eben in ber 21potheke verfdafft hatte.

Der Sterbenbe war balb gänzlict vergeffen. Nod vor eilf $\mathfrak{u}$ ) Den, ber anbere unterm Sifde, und ber Doctor yatte bie Aeckgeit, mir mit lauter Stimme zuzutrin. Een: wauf, auf, ihe Tobten follt auferftehn!"

\# Bon biejen bciben" - rief ex bann unb gos die Reige feines Blafes bem einen ber fhlafenben Murmelthiere auf ben Ropf - 》ftebt vor morgen Mittag nidts zu befürdjten. "

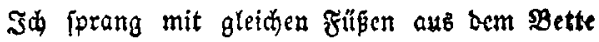
rnb roaf mid in sie steiber. 


\section{0}

ber Doctor vier gelabene spiftolen unb zrei Degen berbei. Dann machte ex fid bab Bergnügen, jedem ber Reiter einen Flügel bes Sd)nauzbartes zu ftugen, unb banb bann ben einen mit ben Füken an ben Dfen,

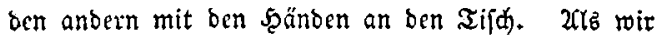
völlig reifefertig waren, löfchten wir nod zum ueber= fluffe bas Ridyt aus und verichlofien bas Stüblein.

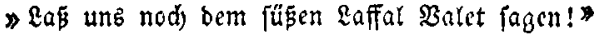

- rief ber Doctor und bielt mich an ber Ireppe

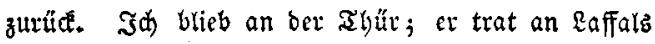
sett, wedite ben Siflafenorn, ber tibrigenz nod nidjt fizen, gefdrwerige benn gehen Eonnte, unb rief

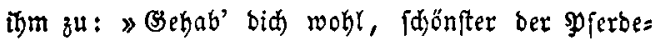
biebe, und willft bu unb narhieţen, fo vergis ja bes Dold) nidjt. Xabe, 5eerz! und leg' dic nun bsin rothtaftes 5ut = Band auf bie :Bunde! " Eaffal fen: bete ibm ein balb Shoce Flithe nad), bod glaub' id), mehr aus Berbrus, in Sd)lafe geftort roorben zu \{enn, alb weil er gerwußt hätte, waz vorgebe.

grsir eilten nun nad bem Stalle, mojelbft auf bes Doctorz $\mathfrak{b e f e g l}$ bie zwei beften 9 ferte fron oullig gefattelt ftanden, jekten unz idhnell auf, nabmen bie 
brei noch übrigen Mifolfferbe an bie \$̧anb und fpreng=

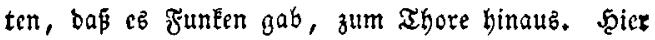

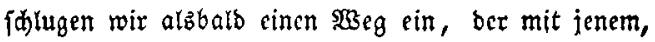
weldhen bie Neiter genommen, in entaegengejester Ridtung lag, und ließ̧en auf ciner gropen Şsiefe, etwa eine halbe Stunbe yon ber Stabt, bie brei Scandpferbe laufen.

Rach zwei Iagen gelangten wir, ohne von un= fern Sdutębricfen (Gebraud) mathen z̆ müften, (über weldhe fen er vorbem berglciden Münzen mit ben läd)erlidiften Eharalteren, z̧u Dut= zenben verfertigt uno um feine Summen verfauft

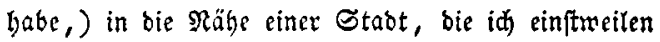

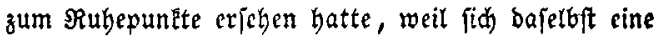
Befak̨ung befant, bcs eolen Ramens ber Solbaten nod) werth uns von einem meiner Eommilitonen befebligt. *)

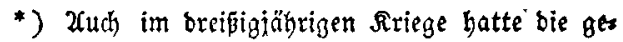
bieterifhe Rothroindigkeit bie $E_{r r i d h t u n g}$ von $B$ üb gergarben verantapt. 26us einigen, im Driginal 
Uts th zuerft bie Thürme gewahr warb, fprang id vom פferbe, warf mid zur Frbe unb bankte

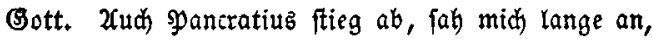
reidte mir bann bie Şanb uno rief etrab bitter, bod)

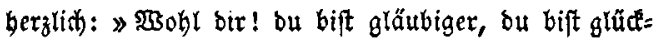
licket, als idib!" -

W3ir wurben von meinem Freunbe, bem Szaupte manne ber sefaz̧ung, auf baz gaftfreifte aufgenom=

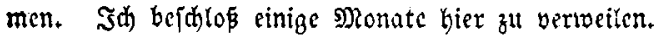
Den Doctor aber trieb feine unrube bald fort. T3sir trennten uns unter ben brüberlichften umarmungen. Ex hat mix fpäterbin gefdricben uno ift exft fürzłlid) als Reibargt, Nativitätfteller und Golbmacher einer febr hohen গ̧erion mit Iobe abgegangen.

\$3ie if felbft aber unter fattfamer Sidjerbeit

berübrten umftänden läst fich fălięen, baß nid)t felten mannfefte Mufenfohne nad vollendeten Stubien Şauptreute babei wurben, - Im allgemeinen wärbe eine getreue Sdjilderung bes Eebens und Sreibent ber beutiden Sodfichüler in früberer seit, von einem Sad) kunbigen geliefert, gewís viel Mertwüroiges uno Erbebentes Darbieten. 
in meine Seimath gelangt; wie id meine alte \$ntter und meinen lieben Rathanael gefunden; wie id meine 9hbltiz zum crften Mal wiebergciefen; wie fie, bas Iüblcin vor bie fhönen fdrwarzbraunen 2utgen bale tenb, Gelle atyränen geweint, uno nachbem bie gotbne Friebensjome in Doutidtano wiedcrum aufgegangen,

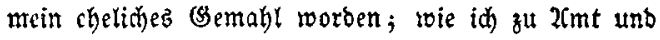

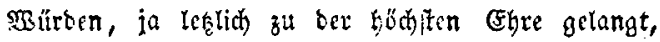

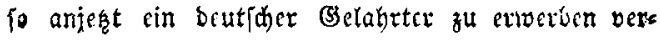
mag*) - Dieß getört nidt in bie (3eịthidjte meiner Sriegs = Fährtid)eriten. -

*) TBSill jagen : zur 2 Uufnahme in bie Duthlauth.

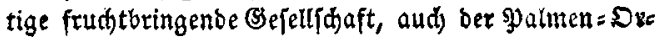
bin genannt.

\author{
F. Sin 8 .
}


Die Graber De foscolo, von

Ernftigr. yon ber Tintobur.

Die Sepolcri deb Uğo Foscolo, der alb Berfaffer Der Letterc di Jacopo Ortis unter unz belannter ift, wurben im Saljre 1806 gebidatet und 1807 zuerft, feits bem aber öfter, in Stalien gebrukt. Sie veranla $=$ ten, als einen netten Ion in ber italiämijichen Spoefie verfudenb, Iebbaften אampf in sob und Jabel. Gonberbar vermeint ein Girolamo Borguo in einer langen Differtation an bem Eebichte barallthun, baß Diefis bie rahare lyrifhe spoefie fey. Pindentonte bat in einem Gebidjte gleidyez ritela, aber ungleis 
d) Sraft, geantwortet; cin butter Diditer, Torti,

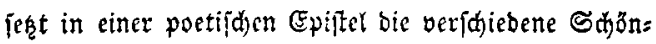
heit ber Gräber bes Foscolo und bes sindemonte

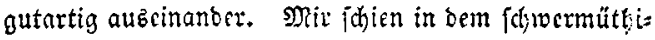
gen Ione bez Fobcoto eine Berwantifyaft mit Eurb Bnon und Eamartine, und, unbefdabet ihrer befon= beren Süarcen, cin 3ujammentreffen brei fold)er (scis fter in brei verịchicbenen Ränocen mertwüroig.

\section{An Sippolyt Pindemonte.}

Db fich ber Sdjlaf, ber cijerne, deb Tobes, \$ohl fanfter fdyläft im Sdjatten ber (5ypreffe, und in ber urne thränerquidetem Shoope?

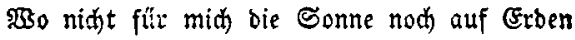

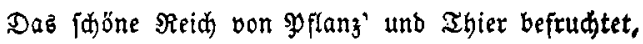
\$3ann nicht yor mir ber bolben 3ukunft Şoren Den Reigen führen fŏmeta)lerifđer Träume?

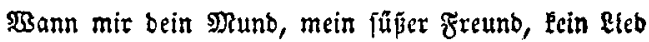


I56

Beberrid)t von fanft melob'joer arauer, fingt,

gann mir ins 5erz nidht mehr ber Geift ber Rebe,

Der jungfräulid)en, trauten Muifn fóndut?

Du einz'ge Seete meines irren febenz,

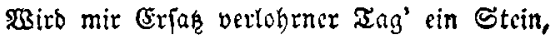

Der mein (Bebrin von unzäbrbaren fócibet,

\$3omit ber Iob fo Eano als Meer bcjät!

Wabr ift es, Pindemonte! aud bic Şoffnung,

Die leşte Böttin, flicht bie Grüft'; es becket

In feine Radht $\mathfrak{s e r g e f i e n ~} 2$ lúe ein,

unb 2ures ftürzt bie mähtigite (3ewalt

Bon Rattid) zu Raufd) Gintaumelno in (Exmattung;

Det Menfd), fein (Srab, fein lestes $\mathfrak{B i l b}$, die Refte

Don Ero' und Simmel find ein Sherz ter Beit.

Dod) warum foll, wer fterblid, bor bee 3cit

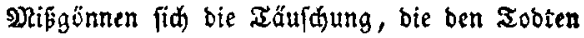

Dieffeits nod bălt ber (Gränge ber ßernid)tung?

Rebt er nidft fort in tiefen Grund ber Eroe,

MBann ibm verftummt bes בages $\mathfrak{p a r m o n i e , ~}$

sann ex fie roeden mit bolbjel'gem zeibe

Im (Seifte feiner Rieten? Scimmlifo, Gimmlifa

3it ber Bertehr ocr liebenben (Gemütber, 


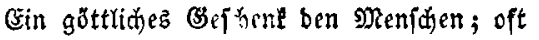
Eebt man burd) ihn mit bem verblid)nen Freunde, und er mit uns, wenn müttertict) bie (Frbe, Die ign als Sind begrüpt' und pflog, nun ihm Zn ihrer $\mathfrak{B r u f t}$ die leģte Freiftatt reidht, \$3飞enn faine Şüllle heilig fie bchütet Bor Sturmes unbill un gemeinen Fußę Entweigender Berütrung, renn ein Stein

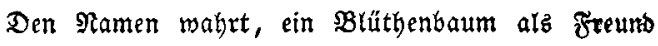
Mit Duft und Sdjatten jeinen Staub erquicit.

Nur wer kein Erbe Ginterläpt ber Riebe, Entbehrt ber turne Ruft; und blidet er jenjeits Der Tobtenfeier, fieht ex, wie fein Beift

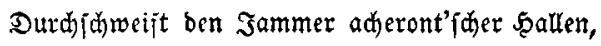
Dber (id birgt im Sd)irm ber mäđt'gen $J$ lügel Dez göttlictlen (Erbarmers; boch fein Staub Berfällt ben Nefifeln unbebauter Sdiolle, Wo Éiner Eiebften Inbrunft für ign betet, Sein einfam wandelnber den Seufzer bört, Den uns Ratur vom Tobtentügel haudst. Dod) Geute legt ein neu (Befés bie Bröber Žern frommen sBlicten, uns befámpft ben Tobtom 
I58

Den Ramen not). Uno fonber (sruft liegt ba

Dein priefter, o Iyatia, ber, bit fingent, Wit langer Riebe unterm armen Dade

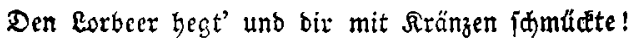

uno bu, mit brinem fähtheln, ziexteft ihm

Den Sang, lombarb'jidem Sarbanapal herbe,

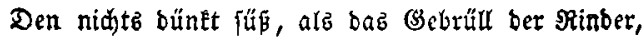

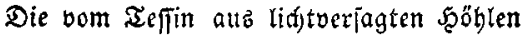

Mit Trägheit ign und fetter Noft beglüdern.

$D$ \{döne Muje, no bift bu? Nidut fügl' id

Zmbrofia's Şaud), Den אünder beiner Bottbeit,

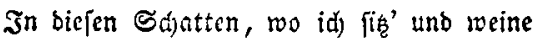

um meiner seeimath 5̧erd, şier fameft ou,

Fier ladtefit bu itm unter biejer Rinbe,

Die tief ibr Szaupt nun nieberjentt und falauert,

Denn, ad)! fie bectit nididt bas Gebein beb Zaten,

Dem einit fo milb fie Sthatten bot uno $\Re u h e$.

Spägit bu viefleidjt, umirrenb unter Bräbern

Dez গöbelz: too es fdjläft, baz beil'ge ફ̧aupt

Deines Marini? Niđit in ihren Mauern

Pflanzt' ihm ben Baum dic Stabt, bie üpp'ge Xmme

Entnerbter Săngex; keinen Stein, Eein \$Bort 
Sat fie füt inn, und fein Bebein vielleidyt Eiegt blutgetränft vom J̧aupte bç Banditen, Der auf baz Rab floct Rumpf und Miffetyat. Sord)! Durdf's Berüll zerworjener (Serippe umirrens, rajdhelt bie vertaffne Şünsin, Stört burd) ber (Gruben Radht unb heult bor Şunger; uno aนb bem Schäber, wie ber Mono floh, id)lüpft Der şiebehopf, und flattert burd) bie Nueuke,

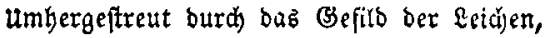

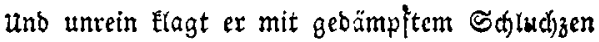
Die Strahlen an, baßi milb nocij ferj'n bie Sterne Den Grüften ber $\mathfrak{S e r g e f f e n e n . ~ D o u ~ f l e h e f t ~}$ umionft, o Bsottin, $z^{\mathfrak{u}}$ ber finftern sad) Um Ihau für beinen Didter, $2($ h) ! keine slame Eriprieft bem Tobten, wo fein berz fie begt, Sein Nund fie preift, feir Lrug' thr agränen ipendet.

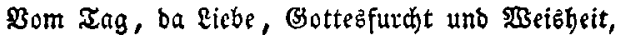
Men[d)lid gemad)t bie menidylid)en şyänen, Erbarmens mit fid felbft und 2 noern, bargen Die Rebenben vor witbem @્surm und (Seyern Die jammerberyen Irümmer, fo Ratur शad) ero'gem Spxudy für anbre Elooje reitht. 
ICO

Die Brüfte gaben 3eugniß ben Xnnaten, Enfeln 2uttäre, und yon bort ergingen Der Earen traute Sprüd)', und bei'ge Furdht Srjirmte ben (Eibjhrour ob ber Bäter Xid)e. Der $\mathfrak{B a ̈ t e r}$ Iugend und ber Söhne $\mathfrak{p f l i d h t}$ Bereinten jid, , Den frommen Braud zu büter, 3roa mannigfach, bod treu burd) Iange Sabre. Nidjt immer lagen ba bie Irauerfteine

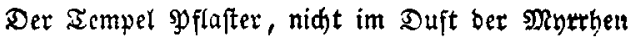
Berlez̧te ba ber Eeichen Mroderhaud) Die Flehenden, nidht ftarrten burh bie Stäbte Gemei Telte Bserippe - Weh! bie Mütter, In ihren Iräumen, jammern auf und ftrecken Die nadten 2 rme zum geliebten Scaupt

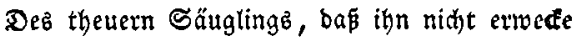
Dab tiefe Stdhnen eineb 2 cugejdieb'nen, Der aub ber uritätt bon ben Erben fleyet Dab Eăuflicje Bebet! - Eypreffen, Gebern, Die reinen Saudbe burd bie Rüfte gicsento, Debnten ein ewig Grün um jene urnen âum ewigen (sebäd)tnís, uno ber giebe

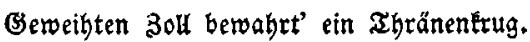


Der unterirb'ichen, Yangen Nadjt zu Yeudjten, Raubten bie Freund' ein (Sluthatom ber Sonte, SBeil nach ber Sonn' ein brecheno 2luge (d)aut, und jebe siruft empor zum forroindenden, (3)liebten \&idt) ben lesten Seufzer jentet. Beftrahlte saffer goffen bie Fontänen 2lts ahau ber 2maranthe, ber Biole, Der bunkeln Sdyolfe 3ier; unb wer ba jap, um Mild) zut weihen uno fein \&eib z̆u Elagen Befdiednen Rieben, fühlte rings um fid)

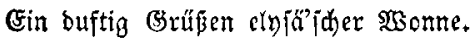
Rübrende Ihorbeit, fo in Zarbion Der Kleinen Stäbte Seügelgärten lieblid

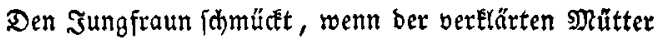
Sebnjudt fie ruft, wenn zu ber Şeimfebre Engeln Sie fleben für ben tapfern Meerebgelden, Der um den Maft fein triumphirenb Sdjif Bertürzt', und fid zur Iobtenbahr' ihn höhlte! Dod) wo bie \$3uth berufner Thaten fidummert, 250 Ueppigkeit und Sdreden find Şandlanger Des Bärgerlebens, ba iff's eitlex פomp, Gebilbe finb's, som Sorkub herbefdimoren, 
I6.2

P3o Fyramib und Marmoriäule ragt.

Daz $\mathfrak{B}$ ole, es fey gelabrt, fey reid, , fey vornegm, Der Stolz, ber (Beift bes berrlid)en Stalienz,

(5) bat zur (S)ruft umidmeid)elte spartőite,

Eebendig fhon, zum cinj'gen Ruhme - 2 appen,

unb wolle sob bie ftille Rubftatt gönnen,

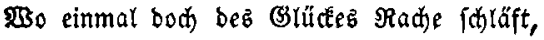

und Freundidaft nidht Des (Soldes Erbe fammert,

Dod roarmen Sinn uno Beipiel freien Riebcz.

D Sinbemonte, zu ruhmröro'gen Thaten

Entflammt ben ebeln (Seift cin ebles (3rab, Berflärt unb weiht bas Ranb, worin cहี liegt,

Dem Silgrim! Jో, ale idj bas Ronument

Sah, wo nun rubt ber $\mathfrak{L}$ cib bes grojen Mannes,

Der Serrifhem inren 3epter nicberbeugt,

Xuftreift ben Lorbeer, uno ben Bölkern zeigt,

Bon weldhen Bgränen, weldem shlut er triefe,

uno Jenes Sarg, bex ben Dlymp zu Rom

Bum zoeitenmal ben Simmlijacn gewölbt,

uno Dé, ber unterm \&uftgezelt bie SBelten

Sab brchn, beglänjt von unbervegter Sonne,

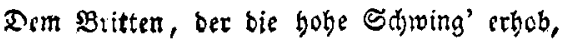


3uerft bie Bahn bes Fimmontentez ebnent: Beglü(tetes sand, rief id), burth jel'ge süfte,

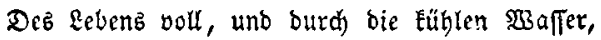
Die apennin bir giept won feinen Cobetteln! Froh Deines Şimmets überifrsömt bei Mono Mit filberreinftem Ridjte beine ફ̧ügel,

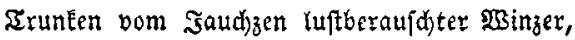
uno $\mathfrak{T h a ̈ l e r , ~ w o ~ b i e ~ w e i k e n ~ S ̦ a ̈ u j e r ~ f t e t h n ~}$ uno buftig bie Slivenhaine grünen, Streun bimmelan ber \$Blumendjöre \$Bseifraud ! Dod) Du, Florenz, vernakmft zuexit ben Sang,

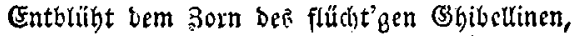
Du gabft bie theuern c5ltern, gabft die Sprothe Dem von Ralliope gefüß̈ten Munbe,

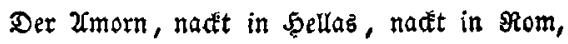
Mit weipipertlärtem Sđjleier zant umhülllenb,

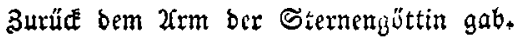
Beglüct're Stabt, bie bu vereint im Iempet Stalienz (3lorien wabreit, adi, vielleidt Die einz'gen, feit bie fdledtbewabrten Zlpen Mit wedjelnder 2almadit ber Menid)enloofe

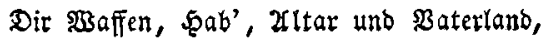


264

unb 2 UKes nakmen, nur niht bie Erinnrung!

Denn wo nod) 5̧offnung leuditen mag bes Rubmes Shodhyerz'gen Beiftern uno Staliens Fluren, Da follen unz bie günft'gen aeiden ftehn. Begeiftrung oft an biejen Marmorn trane Bittorio. Der Şeimath Böttern zürnenb und ftumm irrt' er, wo Zrno frrómt veröbet, Segnjüd)tig blicteno auf (Scfits uns Simmel; und weil nidhtz lebt, wab igm bie Sorge fänftigt, Stand ba ber Streng' uno trus auf feinem 2Antlig Das byeidhe Bild bes Tobes unb ber 5̧offnung. Run ewig ferbft wohnt ex bei jenen 50 hen, unb 》 Baterland" erbebt not) fein (Sebein.

D ja! ein Bott fprid)t aus bem heil'gen Frieben! In Marathon, to Seldenmaufoleen Xthen getwölbt, năbrt' (Er ber (Gried)en Iugend,

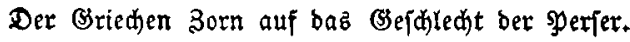
Der Schiffer, jegetno burd) Euböa's Fluthen, Sah Funfen butrdi bie weite Finfterni Bon Şermen blizęen und gejđlagnen Sdwertern, Sah Sdeiterhaufen bunft'ge Ftammen bampfen, Im Eiferpanzer Ririegetlarben ftrahlend 
Sampffertig [pähn; im Braun ber näht'gen Stille Sdrooll weit und weiter, rings Durd bie (Sefitbe,

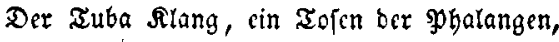
Ein Stampfon pfeiliannell angefprengter Roffe, Der Şuf, Den Şelmfturz Sterbenter zermalment,

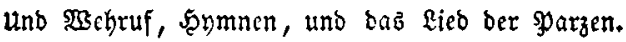

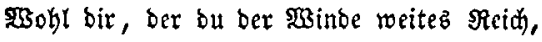
D Sippolnt, in Lebentłcn burdflogeft!

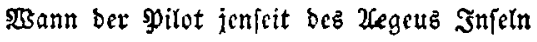
Dein Gteuer Iente, börteft bu gewi Bon Sechthat fhall'n bes Sellefpontor Strande, und heuteno gen $\Re$ böteums Borgebirge Die Branoung zu ठев 2ljob șille tragen Die Nüftung bcs 2 dill. Şodherg'gen Männern

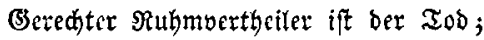
Rcin follauer Baeift uno keine (3)unft ber Aön'ge Erbielt die ftotze Beute Dem Donffeuts, Ė raubte fie Dem windzerjohellten Miel Die vom Zuernus alfijeregte $\mathfrak{B}$ oge. und mid, ben bab Jahrhundert und ber CEgte Durft flüdtitis treibt burd) vieler $\mathfrak{B o ̈ l k e r}$ ande, ZuFrufen heißt bie seclden mich, inr פmujen, 
166

Betebcrimnen fterbliden BebanEens!

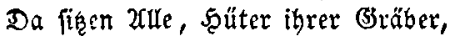

und woann bie seit mit igren Falten flügeln

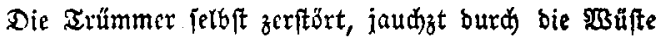

Der פierinnen SGor, und Jarmonie

Befiegt baz Sớrveigen ftarrender 2eonen.

Rod) Geut in Troas unbejäten 2 (uen

Erglänzt ein Drt ben \$ilgrimen unfterblidi,

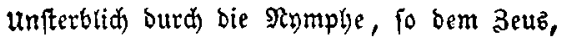
ב̧cub, bem Bemahl, gab Darbanuz zum Sogne;

So waro Iroja, fo watb Xffaraluz,

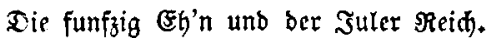
Dod) als EleEtra hört ben Ruf der \$yarze, Go von bez Iagz lebend'gen Rüften fie

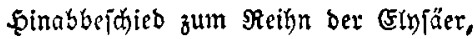
3u Jupiter idjict fie bas lefate Frebn:

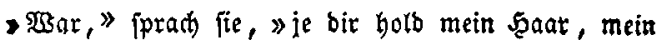
Intitis,

Die (üp burdirwadhte Stunb', und gönnet mix

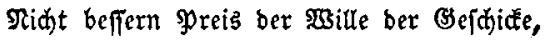
3ur Tobten fieh vom Simmel bod herab Unb Rad)rubm laß Elettren Deiner Riebe." 
So betenb farb fie. Det Dlympier feufjt, (seroährenb neigt bas 5̧aupt, bas himmlija), Bom Seaar Zmbrofía regneno auf bie Romphe, und heiligt'fo ben Reib und igre (Sruft. Dort fduläft aud) (Eridhthoniub, bort ruht Jłub gerehter Staub, bort löften Jions Frauen bas Scaar, vergebens, ad! abflebenb Bon ihren Gatten orohenbes Brrbängniß̈. Dort fam $\Re a f f a n b r a$, wann ber Bott im Bufen Sie fünden biép ben Iobeztag ber Iroer. Den Sdyatten jang fie liebevolles Rieb, Fubrt' ihxer Grbweftern, ifrer sBrüber Sinber, unb lebrt die Aleincn fanfte \$erzenblage. Grieufand fpradi fie: „S, wenn je von 2rgos, $\$ 30$ bem Inbiben uno bem Eaertiaben

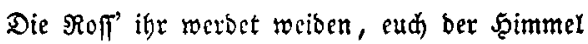
(5)̋̈nt beimzufebren, judfet igr umjonft Der $\mathfrak{B a ̈ t e r ~ E a n s ! ~ b i e ~ M a u e r n , ~ B a u ~ b e z ~} \mathfrak{p h o e b u t s , ~}$ Sebt ifr nod raudhen unter ifren arümmern! Dod wohnen werben Slions פperaten In biefen Situften; ein (Sefhene ber Bütter Şt's, hoben Samen feltit im Elenb wahren. 


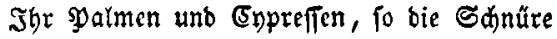
Des פriam pflanzen, ah! ihr wahjet jonell, Beiprengt von Thränen ber verwaiften Riebe. Bejdühęt mir bie EYtern! wer baz $\mathfrak{B e i l}$ Frommen (Semüthz abhält vom bcil'gen Laube, æBirb um slutzfreunbe feltner tratternd flagen, und ben artar mit reiner \$and berübren. Befduüzet mir bie Eltern! Eincs rages Sebt ifr, almojen fammelno, cinen Blinten In euren alten Sdatten irren, fraucheltio um Sräber wanken, uno bie urnen Eüffen, und fie befragen. Dann erfeufzen dumpf Die seb̆hlen all und aules fagt die Siefe: Slion zroeimal zerftöt und zroeinal frimmerno Erboben wieber ob ben ftummen Gaffen, Dał idöner ftrahl' bie leşte Siegrtrophäe Gefeneter speliben. und ber Seber, Der beilige, mit feinem Sang befdrwidjtenb Die Elagereichen Seelen, preift unfterblid Die Füriten ber 2raiver, fo weit Erbe Der grope Bater Scean umarmt. unb Dir, Seftor, Dir wirb ber Shrönen \$̧teis, 
23o als gebeiligt twirb betweint bas $\mathfrak{B l u t}$ Dem Datertanb vergofien, und jo lange Die Sonne leudhtet ob ber Menjhheit aeiben."

\section{Sefief $\mathfrak{n} \mathfrak{n} \mathfrak{e n}$.}

》) Baann mir bcin Mund, mein [üper Freund -》

f. Epistole e Poesie campestri d'ippolito Pindemonte.

》) Den Sang, lombarb'idem Sarbanapal herbe -》

1. 11 Giorno di Ginseppe Parini.

》In biefen Eshatten, too ith fite' -

ber Rinbengain in ber oftiden Borftast von Mairno.

》 unter Brä̈bern beอ \$̧öbels -》

Boritäbtijhe Sottezäctur zu Mailand, two Parini unmütroig bejtattet war.

》 Fin buffiig (Soû́pen - 》

Memoria Josiae in compositione unguentornm

facta opus pigmentarii. Ecclesiast. cap. XLIX. 1. 
170

Ev pupots, antiche illustrate dall' Abate Gactano Marini p. 38 .

$\rightarrow$ für den tapforn Meercabelecn -

Nelfon; ber Naft bes in Gappten ben Fronzofen genommenen Shiffes l'Orient, fein Sarb.

》ber Ieib bes grofen Mannes - $2 c_{+}$》

Macdiavell: Nibelangelo; Balilco, Neroton's

Borläufer; in o. Ritche Santa Croce zu frloretr.

- bez flüdht'gen Ghibclliner - $\rightarrow$

Dante foll sie divina Commedia vor feincr $\mathfrak{B e r}=$ bannung begonnen baken.

- von Rarliope gefüptem Munce -.

Pुetrare rarb in ber $\mathfrak{B}$ itbannung bon florentini= fichen (Eifern geboren.

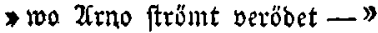

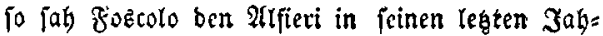
ren. Utth er liegt in samta Croce.

* In Marathon - 2c. "

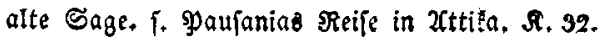


》 Des Sellcipentos Stranse -》

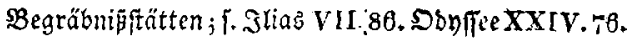

》bie Rüftung bis 2cdill -》

alte Sage. Analecta veter. Poelar, ct. Bruncl,

Vol III. Epigr, anon. 3go. פ̧auโanias, Rap. ss.

》ein Dot - unfterblid -

f. Le chevalier voyage dans la Troade, sde edit. IIawhius ant Dallaway : I.istou's journey to Cunstantinopolis.

"Die Simphe, fo Dem 3eus - 2 "

(Elettra, Iod)ter bes 2ctlas; alte Sage. [. Sd)o

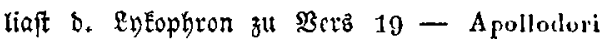
Bibliocl1. L. III. cap. 32, Virgil, Aen. VIII. $136^{\circ}$ Oricl. Fast. [V. 3 I.

》Seht iht - einen shlinden - 》

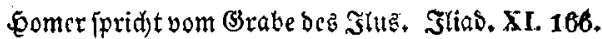
》 Slion zweimal zerftöt $\longrightarrow$ "

bon berkulez, Mindar Isthm. V. epod. uns bon ben 3mazonen. Stiab. IJl. 189. » Sefincter Petiben - Pelidi fitati.

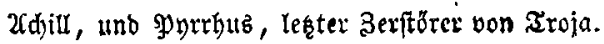


Biprefientrans am હd) Eeftergrabe. *)

$\mathfrak{J m} \mathfrak{B}$ inter 1824

1.

Jib) benfe Dein in trauenten Sonetten, șaie id in ftummer Trauer Dein gedente;

Bern in ber Sdmerzen Tiefe midh verfené, sar ewig Dein Bebilb in mir zu retten!

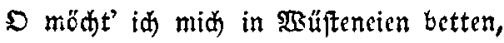
S3o graunvoll liber idroffe Felfanbänęe Strombonner brauft, - benn nur vergebens lente Fil) meinen (S)iff zum Ridht: Fr liegt in Retten!

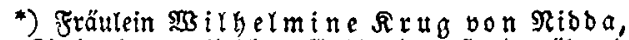

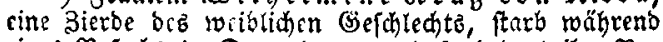
cines $\mathfrak{B}$ c广ú)

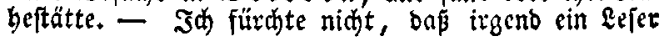


Nimm brum wab Riebesmeh unb bangez Sefnen Sn mir crregt, Bollendete, mit Milbe Bon bem verwaiften Bruberbarzen an;

Sochlyer auz überirbifdem sefilde Send' einen Frictenzftrahl in meine IGhänen, Sđmerzlinberno, wie \$u lebend oft gethan!

\section{2.}

() 2ugen, werth, 2comen zit burdjichatien,

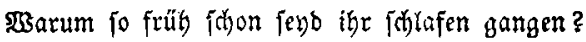
Entziełend eurer Sterne heitres \$orangen Die şamperm [d)liepend untcr zarten Brauen?

Deb Şaarb G'ering', wie Gingegaudyt von 'auen,

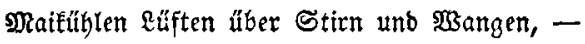
Den jugendtiden $\mathfrak{E} e i b$, ber Didh umfangen, Berftärter (Geift, Dem Iobe idon vertratuen?

Diefe, daz innigfte, heiligfte Gefühf trauernder Bru= berliebe ausjpredienden Somette ben Belegenheitage: bidhten (im hergebradten Sinne beb $\$ 230 r t b$ ) beis zăblen rerbe.

Der Serauggeber. 
174

3roar Dir Ent|d)lafnen raubt' ex nidhtz! Nur \&eben und Seligkeit baft fterbeno Du exworben, Dein Staubgewano bertaujdt mit Serapheftügeln;

2uf Morgenwolken fahn' wir Did entidweben Nur $\mathfrak{i} i r$, bie Sinterbliebnen, fint geftorben: Denn was ift $q$ eben über నobtenhügetn?

8.

2Bobl trugft Du's ahnungšjdwer im weiden \$erzen

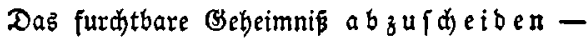
Im Stillen wägent alle unfre $\mathfrak{e}$ eiben um Did, ber Mutter namenloje Sdmerzen!

Uns thmer tiefer brannten Deine Rerzen PWie Sonnenlyeimgang über Dämmerhaiben, und Ieijer nui berübrteft Du die Freuben Der $\mathfrak{x g}$ elt, gewärtig alle zu veriberzen.

Daher mand feuthter Blid in trübe Fernen, Mand Rätbfermort in jarbverflungnen Eauten, Mand' ftille Sdjau in Sulli = unit Wuenorotb: 
Dein früyes şiel, Du Yafeft's in ben Sternen -

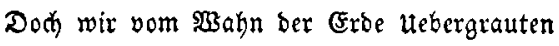
Baucno auf Sąno - bejorgten Feinen $\mathfrak{x} 0$ !

4

- Dort, wo im Felb bie jhlanken \$appelricjen, Fern von ber Scauptitabt raujdenbem Bewegen So geifterartig ihre \$3ipfel regen, Wögt' Эhr einft meinen Sdlummerort exfiejen!

Do mag ber Monb red)t fitbern fid ergiefen, Red)t meland)olifd füße Sdatten hegen, Dort, einer \{el'gen CEvigkeit entgegen,

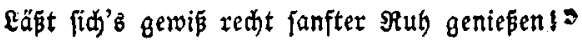

So pradf $\mathfrak{D u}$ jüngft, in Fricben mit uns wallend, Bertlärter Engel! unbejorgt rie nahe Oid) Dir croffneten bie בobtenpforten;

Snbes ber 2lbenbltern vom Szimmel jahe, unb Feierglocen aus ber Ferne forallent,

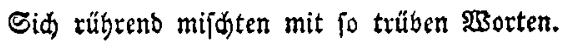


176

5.

Unb năher fam ber $\mathfrak{a} a \mathfrak{g}$, ber unfeilfdroere, Did) ewig uniern 2 rmen zu entrücken;

Die Şlume Deines Rebenb abzupflüten, Spurtos, als ob fie pie gewefen wäre!

Denn auz bez Sđicejals uferlojem Meere

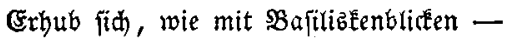
Sein Gift in unjer 2uler 5erz zu brücen -

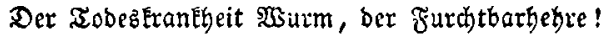

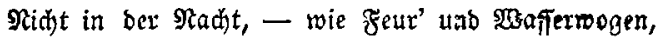
șie Räuber ihre Beute überfallon -

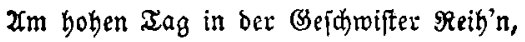

Sam ungewarnt ber Sithrectidue gezogen; Sosie Sebcl über Gonnentyäler wallen,

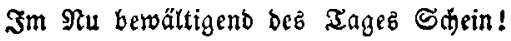

8.

$S$ mehr als Ios : unthätig zu ertragen -

2Benn aud) mit rubelofem :Bziderftreben - 
WBie fich ber grimme Feind am theur'iten Reben Bampiren gleid) ver[augt mit \$ssoblbehagen!

Mit angufenen ofne zu verzagen, \$Bie Blict und Seifter fdion bem Staub entidweben; Die sulfe, fried)end nur mit leifem $\mathfrak{B e b}$ en Die 2ldern mehr burdjidaubern als burdjiblagen.

unb gleid bem şanbrer über Sdyncegefilden,

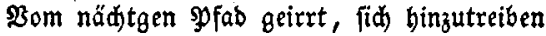
Uuf Blüc und Ungtüa , feben ober Sterben;

Zuz jebem Şügel eine $B$ urg zu bilben, snbeß nur überraufd)t yon büftern (Fiben, unb fdoon umfah'n unendlides ßerberben!

\section{7.}

Dod auth im Zobe nod lebt Bottca Bnabe פुenn aud verhüllt nid)t immer zu ertennen! und nimmer heift er \&eib und Geele trennen, Fr zeige benn bem Beift exft höhre şfabe! 
178

So, §amefter, lam aud Dir yom Riohtgeftabe

Der Eroigleit, ein feliges Entbrenncn

In Şonn' unb Reib, - ein Traumbilo, faum zu nennen

Bon fïns'gen Rippen, fexn vom Reit) ber Snabe!

(Ein (Barten, ähnlid) şarabiefezauten,

Durchirogt von Brumenoüften, Flängen, Farben,

Belebt von Gngeln, wie ber Morgen for̆n -

umfing Didh Shlummernbe in geift'gem Shauen, Did) grüpent : » Selig, bie in Frieben ftarben! Für fie erbłühten bieje heil'gen böbn!

8.

Inb ftiller marb Dein Serz jeit jener Stunde

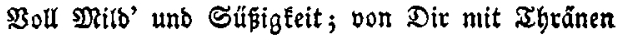
Serübmt, uno jenem namenlojen Gelnen

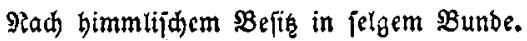

Doh ad, wex fdibert meine Sdmerzenzmunbe:

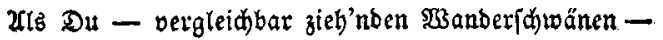

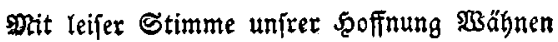
3rratorteft burd bie berbe నobestundel 
Du that'it $\mathfrak{e}$ in ben tief gefühytten $230 r t e n:$

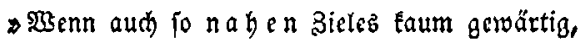

Jd) gern nod) Jabre unter Eudb getebt -》

》 Sdireit' idj bod furdtlob burdy bie bunfle pforten, Denn ber \$erjöhner ift aud) meir geroärtig, In mix vollendend, wab id) nux exftrebt!

\section{9.}

Dort, wo bie bunfeln robtentreuze ragen, Die jene spappeln idauertich umfloren, Die fie zlu ihrem Gdjlummerbain erforen, Sat man fie balb barauf zur $\Re$ uh getragen.

Dies trene Serz, baar aller irb'fden Natagen, 5at tief im $\mathfrak{B l u m e n h u ̈ g e l}$ (id) vertoren Selbft eine Blume, boch aus Bott geboren, 5odiberrlid zu crftehn in Eünft'gen నagen.

Biz bahin rafte ftill in bunkyer Trube

Beliebter Staub! Inbes am wzelten = Throne

Dein (Seift bie friebenspalme längft empfangen; 
ISO

Balb folgt Dein Sänger Dir zur erogen Rube,

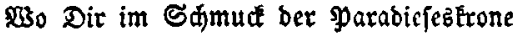

Bercitz baz Gottgeheimni aufgegangen!

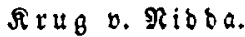

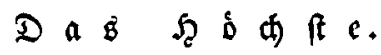

$\mathcal{O}$ unglüc(Ejelig Iradten

in enger Shranten אreis !

- liebeleerez Sかmadten

in Erbenmüh' uno Săveip!

Neinit bu mit lodeern Banben ein langez Blüu zu fabn?

Sätt'it bu bid jelbft verftanoen, ou jăbeft himmelan.

Bom Simmet muß es ftammen, was auf zum Simmel fübrt, 


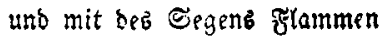
Die farte Bruft boührt.

Ridit aus bor torten Erbe, bes Rebcus Rieterichlag, bon oben tïnt bas 20 roe, bon oben fommt ber $\mathfrak{s a g}$.

und [cine @trablcn zünden, was fid ibm zugeroand, tino in tie Nad) bet Blinden fällt ber Sencitng spfant.

Dann aub bem Dunteln Rerne blufbt ber Bollending Aranz, unb wädift von Stern zu Sterne in immer billerm Bhang.

und Enget freundridi neigen iid) in Scn Rranz Gerein und wollen frine Beugen uno jeine sitier fisn. 
182

Denn nieven Eannô nid)t cnben,

too ewig nux $\mathfrak{B}$ eginn,

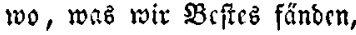

zu Seffrem weijet bin.

Drum breite, fromm erboben,

ber Riebe friügel aub!

bein Soffen iit bout oben,

bort oben ift bein \$̣aub.

Aarl $\mathfrak{f}$ őtifer.

$\mathfrak{P} \mathfrak{G} \mathfrak{a} \mathfrak{t} \mathfrak{a} \boldsymbol{f} \mathfrak{i} e$.

乃3u dem Bilbe ber Erinnerung in bem Eabinet

Der Frau Sergogin Dorothea von Eurland.

(Endlid bab' id wưreflid) Did sepehen,

Wohe Göttin ber Erinnerung,

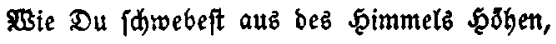

Erwig blüheno, cwig f̧hön und jung. 
Subft auf monbezbcllon Wolfchläumen, Şebft den zarten Schtcier, und Dein Shlid

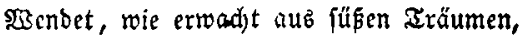

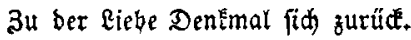

und jo tragen Did ber sbefte Sdutungen Durd) ber Beiten weite Arreife Gin, STso verjunkne Bilber Did umringen, Bleidher Irränze beutungsyoller Sinn. Dod ber Segniudt innigftes Empfinden Wsendet Deinen ftill gejenteten Blice Nod) einmal zu jenen Sdattengründen In ein theures ฐugendland zurüc. unb ein Denfmal [ét' id bort fiá) heben, Daв Zrfádien bie Stätte nennt; Die Erinmung feiert hier ein $\mathfrak{E} e b e n$, Das firf nimmer von ber Geele trennt. Tुseile hobe șimmelz = \&öttin, weile $D$ Erinnrung, weile tröftend bier; Sliebe nidht mit flügetidneller Eile; unire Sreten folgen jegnend Dir. 
I8't

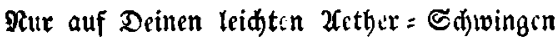

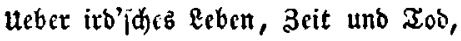

W3agt sex Gsift hinauf, binauf zu bringen

Durch bie Dämmerung zum Morgenroth.

$\mathfrak{u} \mathfrak{r}, \mathfrak{F} \mathfrak{i} \mathfrak{h} \mathfrak{r}, \mathfrak{v}$. Sd dippenbad.

\section{Sdattenbiloet.}

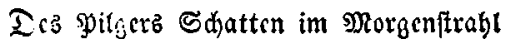
3ialyt weithin butch bie Flur;

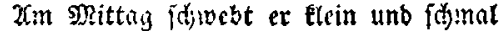

Didlt an reb gailgers Spur;

$\mathfrak{X m}$ Zlbenb nux wäd) it er wiebcr großß, (Ein Rieje finft er bev $\mathfrak{R a d j t ~ i n ~ S d y o o r . ~}$

So zipht ber Jugent Şun[d) unb Traum

Wseit hin ourd) ferne Eand;

Der Mann erblidft bie 5̧ofinung Eaum

In jeinem S(y)itt gebannt;

Dem acter nur Eebret fie wicber zurüct, Sinit is zur aodcenad)t ben Blidx.

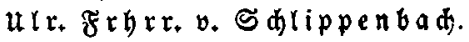


Exd

gandides Spiel in einem 2 ufbuge. 


$$
\$ \mathfrak{c} \mathfrak{i} \mathfrak{d} \mathfrak{c} \text {. }
$$

Robert, cin Jïger.

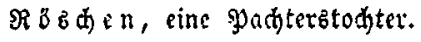

Friti, ihr Bruber. 


\section{Eiffer Aluftitt. \\ \$zalbige Begeno - Niorgenbãnmentng. \\ Robert.}

Dit Singerbuddefe ano Jagotafde - er tedgt einen fleinen Táftg in ber Sznis.

Edaus! ber Boget ift gefangen, Dem ith Zagelang vergebenz unaufbörlich nachgeftellt; Dodi - es madyt mir Feine frecube, Șsil am Sicle bic fez Strebenb Mir ein mädtiger Berlangcn Nach gctietter 2fugenweibe 5eerz und Scele Göher idwellt.

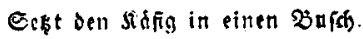

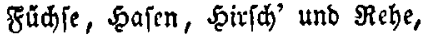
Selbft bie tiefuerborg'nen Dadje

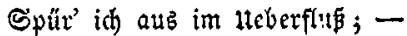
Daß zur Rühhlung meiner Rippe 
183

Mit die rolbe Berr' crroudije

2um verworrenen Seftrüppe,

Sprach ter liebe Sott: Sie roeroc!

unb fie warb nach frinem soillen; -

Fins' idi) auf ter weiten (Eve

Ridt ein Mäbdhen aud, baz Mehe

Diefer beipen Brujt z̆ fitún

Wit getreuer Riebe Rup? - -

Still bavon! wab frommt mein \$ähnen -

Zu' bies Iräumen, all bies Sebnen?

grimmer, nimmer wirb's geftillet,

Benn nidjt eine \$Bunbergano

Mix bab liebe Bitb enthiulut,

Daz, wie fä̈nem Stern entroandt,

Bor bem gcift'gen $\mathfrak{X}_{\text {uge fdrobet, }}$

Dod wohl nixgends für mid lebet!

entrobroticn.

Gort! zum \$̧arbe rajd binein!

Eaut foll mein: ફ̧altog! eridjallen,

Eauter noch bie Büdffe Enallen,

Dap bei frokem T3aibmannzwerfe 
W̧ieberkebre Nuth unb Stärke

Statt ber Sebnjutht berben Poein!

fobrt mtt rer seand aber sie Ettrne.

Zuf! bez אummers fich entiflagen!

Sann it) auti fcin sliseib erjagen -

Frctet Sinn uno frober Muth

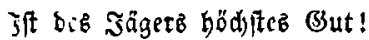

ob int Disticlst.

\section{Zweiter Afutitt.}

Fri

formit von Der entgegengefeytent Selte - Jat eine ewnur

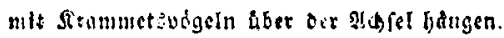

D, wie Elug, baß yor ber Sonne

freut' id aub ben Febern Erod)!

Şüpt mix bod mein Seerz vor şonne,

2Benn ter Gtund' id nux gebenfe,

\$3o bem \$ater zum Seidente

Dieje $\mathfrak{B}$ ögcl id kann bringen! 
I90

vidirlis betrbbt.

Bwar - bas sefte feblet nod);

Denn fold) tobtes 20 getein

Dag ein guter :Braten fenn,

Xber - Eann es benn mas fingen?

lebboft.

Sơnnt' idh bodh an biefem Tage

Einen jungen, flüggen Staar

Dit geídroảg'gem Plaubermaul,

Doer eitte Rad)tigaul

Bon redt rounberbarem Sdlage,

Rur 'nen \$änfling, ber nidft faul,

Reis in :Bater's Bimmer ftellen!

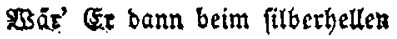

Song ber $\mathfrak{B D z g e l}$ aufgerwad)t;

फorbeno alf ben rüpen Sdall,

Sprấh ex rohl: 》 die lieben Sănger

Đat mein Eleiner Bogelfänget,

pat mein guter. Fitio gebratht!

feutiger.

$D$, iff mollte mit (Sefahr

preines Eebens alle şipfel 
Diejer $\mathfrak{B a ̆ u m e ~ g l e i c h ~ e x ર l i m m e n ~ - ~}$

Sn, Durd) Ströme wollt' (i) jdwinumen;

Sd)wingen mich zum Feljengipfel

Ueber graufe $\mathfrak{g} a f$ erid) lünbe: -

fännt' it) (oldf) ein Zngebinbe

Dreinem guten Bater bringen,

$\mathfrak{u m}$ ign freubig zu verjüngen!

poight oic Dipren.

Still! Wrenn mein Beborr nidt trog,

3irpt es brüben in ben אroned

Jener Sinden! — Dbuohl Fintin

Doer Şänflinge bort wohnen?

fiebt 5imatr.

Roth (Sefieber feh' idh blinten!

Frijh - id wage midh binan!

will ju fletters anfangen und finst, pits et binat fieft.

Iber, ad) - wie fteif und bod)!

siönnt' id ba nidjt ldjwinbelno wetben?

verjlaft.

ख̧enn aud)! - Bater ift auf Erben

W3ohl ber allerbefte Mann -

unb wenn mid) ein (Srauen faft 
J9)

Droben auf sem bohften 2aft:

Dene' id Dein, o Bater - (inniy) lieber!

und ber Sdirindel geht vorüber.

cr Elettert auf ben $2 a$ am.

\section{Dritter Aftritt.}

$\Re$ ő \& đ ก ,

utit einem ndibijen - Erbbetsen fudjeno.

Shon beinah' orei Biertelftunden

Eauf' id ămijg bin uno ber,

Sude frudtlos freuz und queer

פach ben Yeb̆ten \$urpurbeeren,

Sie bem Nater zu bejheren -

SBenig hab' ith nod gefunden;

\$3as mein אürbden jest bejdwert,

Sit wohl faum bes $\mathfrak{B}$ ifdenz metth. -

Spärlia blibt die rotbe Spradt

2ub bem jhattcureidben (Gritn,

Fsie ber einzelne $\Re$ ubin

untet Şưllen von Smarago. - 
Freilid tiefer bort im watb Fannd' id Beeren wohl bie Fữle; Dod bie jouartictse Stille Ift vielleitht ein 2uluenthalt Böjer Dieb' und witber Jãger! Darf to früh idh ohne zagen अud) Den Sdyritt ins Didid)t magen? -

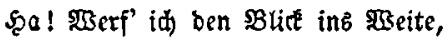
unb mir läd)elt Erober = Sđhimmer Scold und lieb, fo wane' thimmer, und mein Suth wirb inmer reger! 5ab' id nux fo viel bex Beute

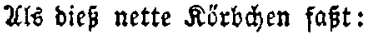
Eüpf' id mit ber füpen $\mathfrak{E a f t}$

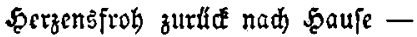
Sdjleide ftill in Batere Rlaule; Sege meine Erbber = Gabe Rämlidh, wenn id̆ fie erft babe Eađite, fadtt' aufs zonfterbret reben Bater's Simmelbett. Deffnet Rater Dann bie Zugen, Xub bem jungen Straht ber Gomke 
194

Rebenzfreuben cingufaugen, und aus milber Sommexluft

Strömt ber Beeren würz'ger Duft

Rieblid) [dmeid)elns ibm entgegen: -

D, fo füblt jein Seerz mit 2 bonne, Dap fein treueb Rinb es war, Beldhez ihm bie Sabe brahte, unb bes fdönten rages bad)te, Der ben Bater einft gebar, und - id benfe mir's mit $\mathfrak{e} u \mathfrak{f}$ Xn ber bäterlichen sruft

Birb die Freube mir zum Segen! jufammenfalient.

Xber - bord)! bort in ben Sträư⿱⺊口)en Raujht es, wenn id midh nidbt thöre Sott! mit blantem Morbgerdebre

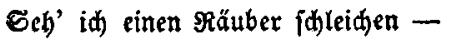
Suter Sott! erbarme bid -

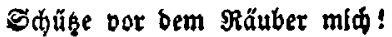




\section{Bierter Aluftitt.}

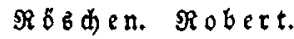

Robert, ans bem Bebrifs tretent - aberrafor.

Golla! he! wab finb' id hier?

Sold) ein zartes 2 3ill erbliate

Nie mein 2 ug' im

Butes Sagbglük Eünoen \$Beiber! tritt ndber.

Mabden, jag'! was treibft Du bier?

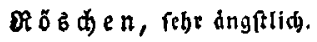

Xd), Beehrtefter ఏerr Räuber!

2(d), id bitte, thun Sie mir

ふa bezroegen niđhtz zu Reibe,

Beil id ein paar Beerden pfladte!

Robert.

Fürd)te nid)tż, mein gutez Rinb!

Mäbden, bie fo lieblid finb - 
106

faft thre sant.

Dexen Eand to weid), wie Scibe,

Deren şangen frifh), wie sojen,

Dercn Saden wrip, wie Sanee,

unb umwallt bon braunen \&oden -

Fold ein zartes, zahmez $\Re$ Rh,

Yllegt ber Şaibmann liebzuẼofen.

Ei! warum bean fo erjdroden?

$\Re \mathfrak{0}$ ह क ค $\mathrm{n}$.

24)! if badyte mir mit (Braufen,

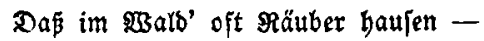

siobert.

Siebft mid frommen Iägersmann

Du fứr cinen säuber an?

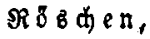

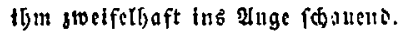

245o - Sie find witelich fciner?

Robert.

Thie Man's nimmt. SWobl bin ith einer;

Seit idy, Mäbden! Didh gereben, 


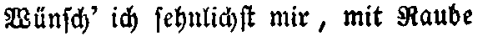

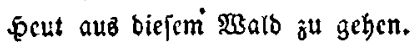

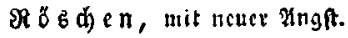

wesb mix!'

Robert.

Ruhig, fanfte Iaube!

Sieg nur, Mäbden! :əab id beute, Xrł bom Bräc bejdic'ne Bette,

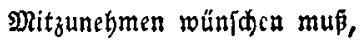
Jit -

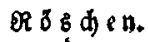

S Bott! und wab?

Robert.

હin গิแ下 -

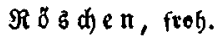

Mcitex nid)ta ?

(id) Dlóglid indumetro.

\$3arum nidht gar!

Robert.

3it bei Dir ein $\mathfrak{A u}$ jo rax,

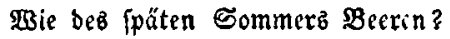


I99

$\Re$ ô ริ (t). $\mathrm{n}$.

Das juft nidit. - Ein $\mathfrak{A} u \beta$ in Egren

Scy bom licben Sott erlaubt,

Sagt bie Mutter; bod gcra $\mathfrak{u b t}$,

sft er - unredt hier zo Eanbe.

Robert.

acber wer, wie id, gefttet

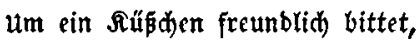
şiulit $\mathfrak{D u}$ ben bez $\Re a u b ' z$ verflagen?

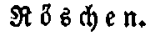

$\mathfrak{O}$ ! vor be m foll man exf zagen! Sagt bie Mutter - wocil, wer $\mathfrak{B a n b e}$ un bie Gerzen bittend legt, (5wig unb in Feffeln fđlägt.

Robert.

Sind ja bod nux $R$ o fen banbe -

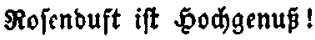

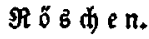

glun wohlan! wenn bis zum Ranbe

Gid mein Sörbchen fült mit Beeren: 
Wrill id grgen cinen Sup

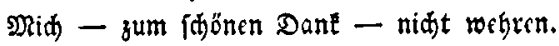

Robert.

(S)ut! idh weiß bier jeben Flcd, und senn idi) bei'm Suden belfe, Sommen fĭneller wir zum âned, Du - zu Seer'n im Ueberflúpe, Эक) - zum (d)

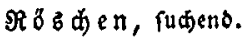

Damit hat's nod lange 3eit!

Robert

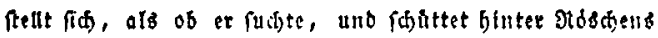

Tüfen aus feiner Sagdtafde Erobeeren in ben Forb.

Nun, wer meip! wenn unb ein Elfe

Diejez Traldes Bunft verleigt,

2Bobl uns 3auberfitüts fpendet,

Wrirb bas

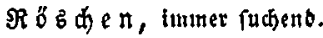

Ei, wenn wir auf Geifter lauern:

Sönnt' es Froigleiten bauern,

Bis mein Förbden ganz fid füllt. 
Sobert.

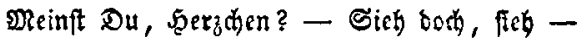

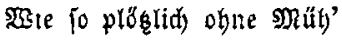

nebern sand bie frudt fidon quiat!

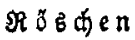

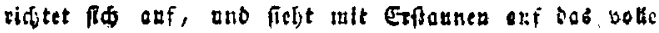
Stdefaten.

thebervolf! - Bott fteb' mir bei -

Das ift eitel Sauberei;

Drauf verwett' id gleid mein Eeben!

nimut Sen Sorb und betracitet Die Beeren.

Robert, (ad)elnd.

Oleid) Drin Ecben? - mollteft Sprci6

Deine foünen rage geben?

Nid)t bod, bolbes Rinb! Ja weip

Einen beffern Sabs bafür.

Du bezaglft zehn Rülfe mir,

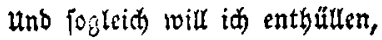

Fsie fid), obne 3auberipiel,

3roanzig forther sidrbe füllen. 
$\Re$ \&

(scen licis id) mir's roobl extäran Dod) zchn Siuffie fins zu vier, Hno bie Muttcr fönte forterten;

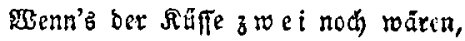
Rum, Der Sandel müdse gelten!

Robert.

Brabo, liebes Mäbdjen! $\mathfrak{D}$ kegeft mir im banbel zu, und id laff' ein wenig ab,

Du fteigft auf, id geb' binab.

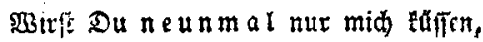

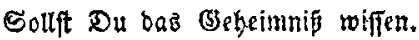

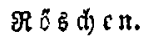

Deunimal ? nun, und nimntetmegr!

Sdon an zweimal ging id fdiwer.

ORobert, iddecre.

Xate werd' id boch ertangen

Bon bem Erokecr s rethen Mand? 
202

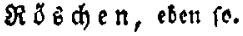

3roei! bie 3 abl ift boll und runb,

3roei, nidbt mebr - uns auf bie $\mathfrak{B}$ angen!

Robert.

Sieben gieb mir!

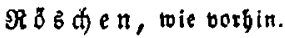

Stäbden liebea

Rimmermebr bie boje Sieben.

Robert.

Gieb mir Sehie!

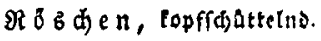

gann nid)t feyn.

Robert.

Ftunfe?

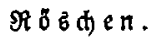

Xud nidht !

Sobert.

Biere? 
$\Re \ddot{B}$ B (ด) E

Rein !

Taufe.

$\Re \circ b \in \mathfrak{t}$, nad einigen Einmen.

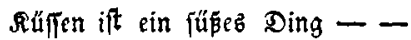

Sind bie 5erzen aud babei,

3ähyt man guter Dinge orei!

Sich, wie jener Sdimetterling

Dreimal auf bie Blume fintt,

Aüffend ibren bonig trinft; -

Uนd) bas $\mathfrak{B l u ̈ m ( j ) e n ~ - ~ b i n g e r i f f e n , ~}$

\&äpt fid breimal witlig Euffen!

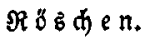

Ja, baz bat aud Eeinen ssillen, Sann nidte weigern, nod) erfüllen, Führt ein traurig $\mathfrak{Y}$ flanzenleben, Sann nidft $n a$ d) bem flattrer jdiveben, unb er ferbit - mit leidftem Sinn, Eilt zu jdönern Blumen bin. 
Sobett.

Unbers füblt ber treue mann, Sudt bie gleidgeftimmte Geele,

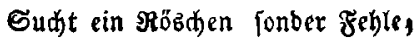
bat er bas gefunben, bann Blügt für ifn ber sBlumen teine, Alв bie Rieblithe, die Seine!

$\Re$ ơ है की $\mathrm{n}$, trociftul

Xber wie - wonn 2 sinters \$aud)

Run bą Brümdhen reift bom Straud?

Fobert, innig

2rsitb ex berzlid mit ihm leiben;

Mit itgm welten und verfheiben!

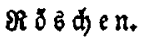

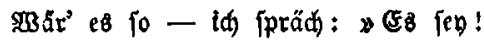

Xute guten Dinge brei! $》$

Robert, feurly

Sag' ez, Smbdan - fomm und zafyle!

or will fie foffen. 


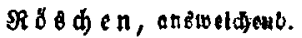

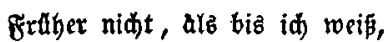

W3ie gleid einer Bauber [d)aa?e,

Ohne Müg' unb obue Fleip

Sod mein Forb von Becren fibuoll.

Robert.

Retche ben betung'nen 3oll, unb Du wirft jogleid belehret.

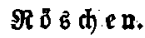

Retn, ডetr Săger! umgekehret -

Erft bie Waare, bann ber Preiz!

$$
\begin{gathered}
\text { Sobert, } \\
\text { nutt fanftem Bortuarf. }
\end{gathered}
$$

Eng fưhl' id mein Serz umiponnen -Sabe Did fo lieb gemonnen;

Soffte erft, baz zarte Reis

Sunger Reigung, bann Bertrauten,

Dir im Şerzen anzubauen,

Ireulith, biz all' meinem Mîthen

Begenliebe würo' erblüben 3 -

2lber plơsclid fällt ber İhan 
200

Deines Miptrauns auf ben $\mathfrak{B a u}$ Neiner Soffnumg Ealt herab, unb ftreift alle Inofpen $a b$. -

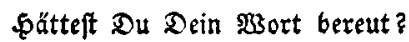

$\Re$ อ̈ В bewegt utib ben Briaf asu Boben.

(Ei! wenn id aนs Sittjameit (Bleid) mit (Sleidjem nur vergelte,

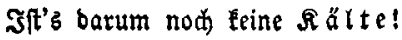
Robert foft if the şand mit forter.

Darf ih hoffen - barf idus wagen? Bift $\mathfrak{D u}$, jiffer sind, mir bolb?

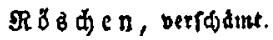

kies id mix nid)t ab 伤on fragen, \$Bas id nimmermegr gejollt?

Robert.

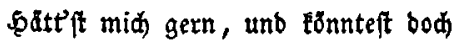
Begen einen Siup Did) jträuben? ex umfaft fie jodrtli申. 


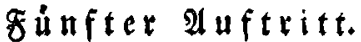

Borige. Frik.

Fri

Der ftion etroos frủber bom Baume foralig, trilt fasuell

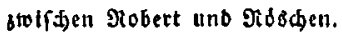

Eafl' ber werr bie syoffen bleiben!

X̧agt Er bas nur einmal nod),

खुers' id'b -

$$
\text { Robert. }
$$

\section{พ3at?}

Fri

Dem Bater melten,

Brab id bout nom Baume fab!

Robert, tadst.

Geb̆t mix bod ben Heinen forthen!

$$
\text { frib. }
$$

Эa, id) (aufe, wie ber Blis,

unb bann ift bas unglüd ba! 
208

$$
\text { Robert, fidttist. }
$$

(B)

Sprid! wer bift Du, breifter Bubr?

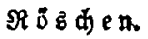

'b ift mein jungfer $B$ ruber Frio.

robert.

E. Dein Bruber?

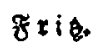

$\mathfrak{J a}$, bie gute

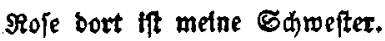

$$
\begin{gathered}
\Re \text { obert } \\
\text { tritt ndfer ju frts. }
\end{gathered}
$$

unb nad troggf $\mathfrak{O u}$ ba im Sute?

$$
\text { Fr隹。 }
$$

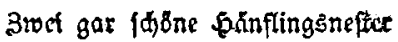

Sit fünf allerliebften Jungen.

Nobert.

SBle btit 3u bazu getommen? 
Frik beutet auf ben \$aluu.

Şab' fie Sroben altsegenommen -.

SBin von 3weig zu 3iveig geiprungen;

şar fdour lang' auf ihrer Spur.

Robert.

unb was trägit $\mathfrak{D}$ u an ber Signur?

Frif, betgnigt.

Dreizehn fette Arammetzob̈ger!

Reicht bie erizten, bie getöbtet,

Spit bie (Fibijd) = Beer fidh röthet.

Robert.

Sabmft fie bort wohl aub ben Shlinger?

Fri $\mathrm{ic}$.

Flugs bem $\mathfrak{B}$ ater fte zu bringen!

Robert.

2rifo baft, nath \$rildobiebriegel,

Reft und $\mathfrak{B o ̈ g e l}$ Du geftoblen?

\&ri $i$,

oreift oor Pobert gintretend.

फert! foll id ben $\mathfrak{B a t e r}$ bolen?. :. ordet bie gebaute zante auf ben fupf. 
sald wirb mir bas Ding zu fraus!

Bater nennt fid 2(nton Straus, \$obohnt im Dorfe Maienthal;

Jit ein reltberübmter $\mathfrak{y a l d t e r , ~}$

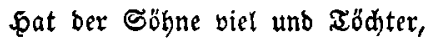
unb kein Cing'ger ift ein Dieb; Drum, hat Er bie Raje lieb, Sag' (Er bab nidht nod eimmal!

\section{Robert.}

Rur gemad), mein fleiner becld!

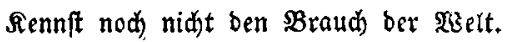

In bem Maienthale brüben

$\mathfrak{M a g f t} \mathfrak{D u}$ plünoewn nad Belieben;

zcber wen im Forftrebiere

Эd) alz $\mathfrak{B o g e r f t e l l e r}$ fpüre -

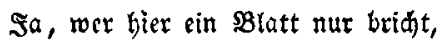

Dber tobtet einen \$surm,

Sifit verfallen bem Sericht.

Du baft zwiefach gar vericęet

Serrid)aftiches Mad)tgcbot;

83 irft in einen finftern Zhurm 
Rur bei $\mathfrak{B a f t e r}$ und bei S6rob arjogleid von mir gejezzet.

$$
\text { Frite lo: }
$$

Nidyt nur in bex alten Stabt Rürnberg fängt man leine Diebe (E)' man fie gefangen hat; Sirin, man fdiabt alta) hier zu Drt

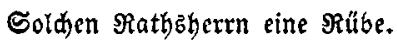
ralabt gi:bdjen und so ill fleben.

Giegt ber ferr - id laufe fort! Robert.

Şilft Dir nidtz, bie Snabenfinte! Magft $\mathfrak{D u}$ aud im Borfprung feun Sichft $\mathfrak{O u}$ bie gelab'ne Flinte? 3hre אugel holt Didh ein.

$$
\mathfrak{F} \mathfrak{x} \text { is, Angftidy. }
$$

Bott! Sie werben bod nidht fatesen?

Robert.

Must für Deine Rectheit buficn! 
Frig Enict nicoer.

2(d), Bcrzeibung - bitte, bitte!

Senne ja nidht :23aldes Sitte;

Sabm mix, alb ein barmlob Rinb, Dhne $2 \mathfrak{r r}_{\mathrm{g}}$ es mix zu benken, Reftre ba, wo Bäume fino;

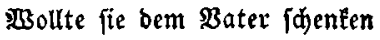

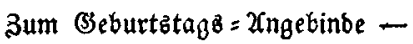
Darum wenn $i d$ armer Snabe \$3inflid) was geftohlen gabe: $\mathfrak{D}$, fo madte Mindesliebe uno nicht Şabjưd)t mid zum Diebe; : $\Re$ obett.

(3)ut, mein Eleiner Freund ! Dix if aule Strafe für bie sefter und bie Bögel ganz erlaffen,

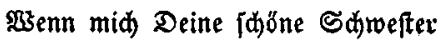
Oreimal red)t berzinnis füpt. $\mathcal{F} \mathfrak{i}$ is, frof aufitebeno. $\mathfrak{D}$, wenn'? bier nur Aüffe braudit, um bei'm ছerzen Sie zu faften: 


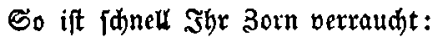

S u n bert Rüffe will id geben,

Rann's ber Strafe mid) entheben!

Robert.

(3)laube Dir'b aud) ohne Sdywur!

2bber, Eleiner Sdäler! wilfe,

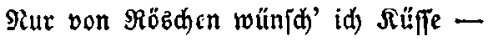

Rözden smuno befreit Did nur.

$$
\mathfrak{F} \text { ritic, ftaunenb. }
$$

Sdau - bas find' id wunberlid!

Bater fagt bod), Rop uno id

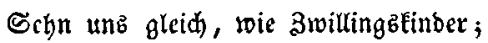

Wrarum foll mein $\mathfrak{f u}$ benn minber,

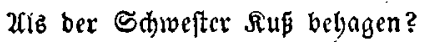

Robert.

รa, baz läpt fid) nidt fo fagen -

Füflen nur; mir bits' id cin,

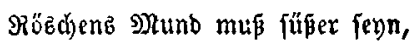

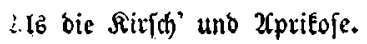




$$
\text { Fri }
$$

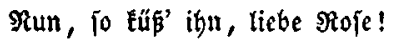

Das inm beutlid) wero' uno Euno, Ririd' (ey Rixide, Muno (en Runt,

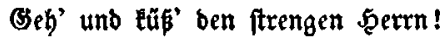

$\Re$ ถี nabet ficf Robert fefr idjambjaft.

Soll's benn โenn -

Robert, jirtlids.

Dou thuft's bod) gern?

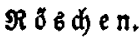

Srcin!

Robert.

พ3axum nid̆t?

$\Re 8 s$ d) $\mathfrak{e}$.

Mund und :range,

Sagt tie Mutter, reid' nur (Einem

203illig bar ; jebod im 3range

Reide Mund und \$ange Ninem. 
Robert tritt jurde.

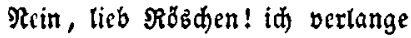

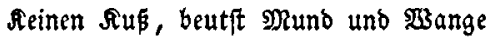

Du nidht bar mit freicm Sinn.

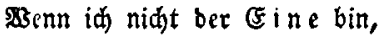

Den bie gute Mutter meinte,

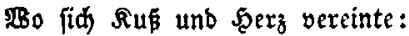

Ud)! fo bin id für Did Siner.

$\Re$ ö z d) $n$, fĭr fid).

Ud ! !

Robert.

bat ben adfig alls bem Bufife genoumen - jul Zrik.

Nomm ber, mein lieber Aleiner!

Nimm bier dief́n jungen Staar;

Bring' ifn Deinem Bater bar

Ifm Geburtstag' zum (SejđenE,

und fey meiner eingebenf.

$\mathfrak{F r i s}$

bipft froblyid umber.

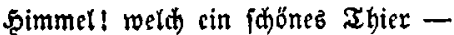

Dank, \$̧err Säger, Dank bafür!

fifGt Nobert. 
216

Robert.

Reiofft Du brinem Staare Futter, Zränfft ibn aus bez Felfens sBrommen, und ber $\mathfrak{B o g e l}$ madht bir freube: Sag' bem פater unb ber Mutter Dape ein wacker $\mathfrak{J a ̈ g e r ~ l e i b e , ~}$ Seit ex $\Re o ̋$ djen lieb gerwonnen, unb verjdymäht von sősden war! Rinbertreue wollt' ex üben an bem buten Erternpaar,

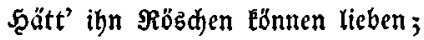

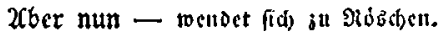
tecb mohl, mein Eeben -

Freube witb mir nidhts megr geben! er toill fort.

$\Re \not ̋ b$ क $\mathfrak{e n , ~ r a c d ) . ~}$

Jager - balt! - $\mathfrak{O}$ u bift getrántt? Nomm! Mir fagt $\mathfrak{c b}$ mein Bewifien: Fser ber (Eltern herzlid bentet, Den barf röbden herzlid füfien! fie Eñft robrut. 
Sobert.

Sit es :Babrbeit, was id füble?

(Slaubte mid) vom fäbnen Biele

Rod) entfernt, wer weip wie weit;

Dod) verbürgt mir Geligecit

Jez̧t ein Rup füre ganze Icben!

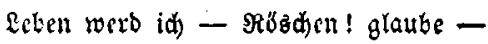

গur burd Did, und nur in Dir!

berjinuig.

Sichit Du, Rins! mit fübem $\Re a u b e$

(3el)' id bennod jebt von biex!

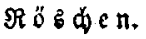

Rein - mit nicoergefd) lagenen Itagen. was ith geipendet babe,

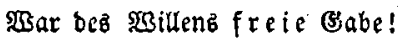

sobert

umfaft fic játrida.

Darf bann aud Dcin Robert hoffen,

Folle cr Sexzenz Serz getroffen,

Mebr Der Rüfie zu exiagen?

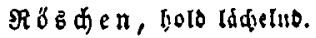

Robert má bie Eltern fragen. 
Robert.

unb ift Elternjegen ba? -

$\Re$ ถ้

Sagt bas perz bes Serzens: ja!

Robert,

ife feelcnooll ino Iuge blifent.

2 ohl mix, wohl! Jd) bin ber Eine, Den die Mutter meint, ber Deine!

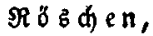
den $\mathfrak{B l i d}$ errotedernb.

\$Bobl mir, baß mein fanfter 3äger

Riđht cin willber Räuber war!

$$
\text { frif }
$$

reidet Robert sie Sart.

Ş3obl aud mix - wix werben Gdräger, und (a) bab' 'nen jungen Staar!

Robert, dn Rdschen.

Sag' mix nod, Sou herzig Rind!

Db bes \$ralbez Spurpurbeeten

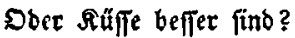


$\Re$ oै है d) $\mathfrak{e} \|$, errdtbent.

Ğe!m! baв mus bie 3utunft lehren -

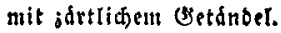

Эăger nennt/man jhlimme Gäfte!

Robert, eben fo.

Xud boi unjerm Sodzeitfefte?

$$
\text { Fri } \mathrm{ig} \text {. }
$$

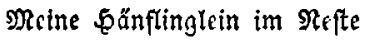

unb ber Staar find bod baz $\mathbb{B}$ e fte!

(D) $\operatorname{cotgant}$ fatt.)

6. R. Soltenob!e. 


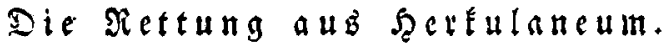

Soyllifdes Epos

oo $\pi$

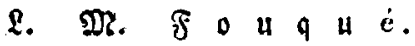

2Beit über ftolze Eapitoliumb = 3innen

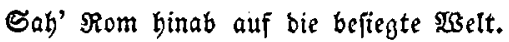

Dod) Rom erbebte vor fid jelbft tief innen, -

(Ein \$radthauz, hart an Rraterz Sdjlunb geftellt!

S3ie barf in Rom ein Suell ber Freube rinnen,

So lange Nero ftraff Die zäger hält,

Rero, ber praditooll blut'ge Zugerandte,

Dem 5ö und auft und Stolz im 5̧arzen brannte!

Nero, ber berbe Feinb bez janften Sinnens,

Daz fehnend auf nad গ̧arabiejen fonebt!

Nero, BeË̈mpfer jegliden Beginnens,

Dab Fried' uno Şuto im Erbenthal erftrebt! 


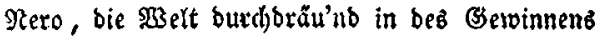

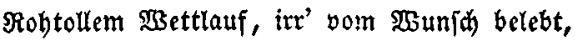

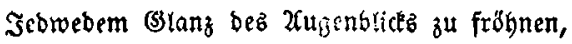

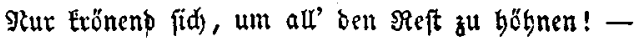

Rero gab gräßtich nag' uns fern Beferse, Ncin, nid)t (Bejef̧e, Traumebgrillen nur. Sdjien's beut, baß wer ben Feind verfolgend beae, şarb morgen Der zum গsilo, beß blut'ger Spur

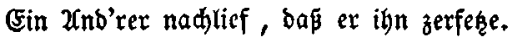

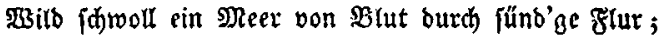

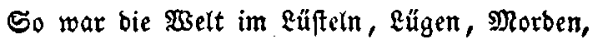

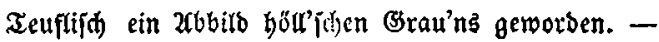

Dod) mag bie \$selt rings wüthen ober winjeln, Stetz findet irgenbwo bas ftrenge Recht Sid) feftgeftemmt auf Alippen ober Snien, und irgend nod) bereit zum Siegsgefedt. Sey's nun mit Sithern, Sd)werdtern, Meifeln, Pinfern, şa äd

So audh ergab in \$̧ertulanum'z Mauern (Fin Sängling, fdrweigens nod, fid eblem Irauen. 


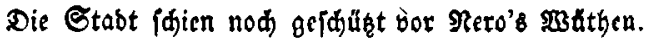

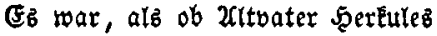

Bon ibren Binnen ricfe: 》 wenn zu hüten

Wier Einez $2 \mathrm{~cm}$ ift, -

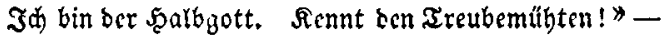

Dod) jener Jüngling, Jucibiabez

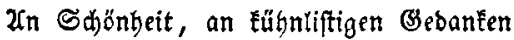

Ighemiftotlez, trat weiter in bie Sduranken.

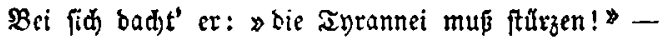

Bon aufen zeigt' ex fib alb sitherfreund,

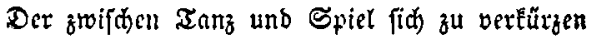

Des kurzen Rebenz Rennbahn jey gemeint, Mit Poefie, bem Field voll stier :Bürizen, B3or bem bas Menjhenfinb, fonft berb verfteint, Sid) burd ber wunberjamen $\mathfrak{a}_{a b e}$ Spendung Belcb' uns förb're zu bez Scinz Bullenbung.

Inb holb octeint' ign füpe, keujde sicbe

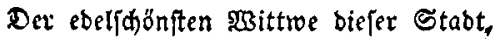
DIympien, bie mit unverhebltem Iriebe Die reine fand inm gern geboten bat. 
Man ftaunte nur, weshatb nod) unterbliebe Das Şymensfeft, um zarter Myrte SBlatt

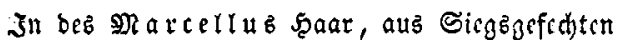
umlorbeent/jon, verjdjönenb cinzuflediten.

Marcellus aber hatte bie Bebanken Der kühnen $B$ ruft Sinmpiens längft vertraut. Sd)on fah' im (Seift fie ben Inrannen wanken, und wollte nidt, von \$olken nod umgraut, lint Scodizeitfeft in bangen Iräumen id)wanken, Rein, Har und froh, arz bes Erretters Braut,

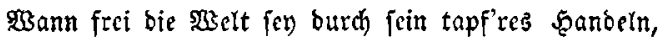
Un feiner 5and zu \$umenz Zempel wandetn.

Die Stunte idien zu nab'n. Borfidtig immer, Dod) ftete aud) fưhn, fab' er sesci'bn bie Eaat, Durd inn geftreut, wie unter'm 3auberfoimmer

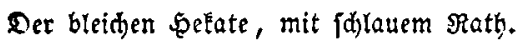
Do trat er einft zornglühenb in bas 3immex

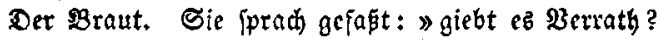

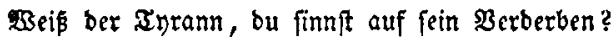

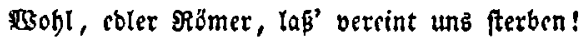




\section{4}

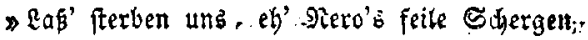

Den Tob une bringend, uns ben aob entweig'tr. Sief) bier ein hailam (Sift; bas foul uns bergers

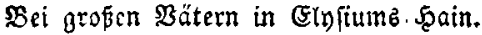
Nan grub ez aub bez Riluz Ronbesbergen,

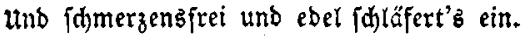
şsoblauf, mein Römerheld, und laßs' unz eilen! Mit bit will ZUlles, will aud Dos id theilen. -

Er hort fic hodibegeiftert. Fhre Borte Berfiegern ifm ben Mund in ftolzer Ruft. Nur als fie ject, begierig nat) bem ફorte Des Iez̧ten 5̧afenraums, altb zarter Bruft Dab Fläjhlein zieht an gorlogervebter Borte, Dem Mund es nah'no, - ba roits er fein bewupt, (Breift Gemment ihre şand mit feinen beiben, und ipridit: 》nein, Sculbin, fo nid)t follit bu fdeiben!

T3ohI freu' id midi an beiner gropen Seele; -. Dod bie foll leudten nod ber beitern 13 elt.

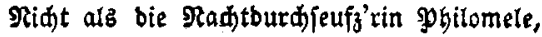
Ulb Siegeslethe, roenn ber Zag ethellt 
Die Ers', unb Radjt entflieg't zur bunten Şb̆hle, Bon Freubenjang bein göttlid Scerz geidwerlt, So jollf bu, o Erbabenite ber Sdjün, Du lidtoertündeno Remnonzbilo, mir tönen!

Ridut brobt Berrath ben rübmliden Entwürfen, Die meine Bruft in fid) verid) offen trägt. ş3enn jeegt fie ungedulb'ge Flammen würfen, Dem Riejen äbnlich, vom Bejuv umbegt, Da mód)te Nero ftolz Falerner fhlürfen! Sein Feinb ift furchtbar, ber eh' broht, alb jdlăgt. Mein \$3ere - burd) Nadit Ginwal'no wie 2(rethufe Rennft einzig bu, o meiner ßyaten Suje!

Rach feinem ganzen umiang, bohem siele, Rennft bu von allen Reniden es allein -

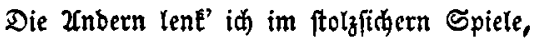
üls faum nur fealbberougte aus und cin. Dod wenn id vor ber legten ahat nun fiele

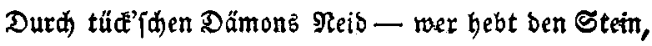
Der unfer Bauweré feft unb ftark vollenbe? Bu zart, zu weiblid fino Dignmpia's șänbe! 
206

So fann id längft, und ftond aud beut fo jinnenb xm Sportiêus, wo fid Sheraflece Bito

șie lebend zetgt, bie grope $\mathfrak{3}$ aht beginnend,

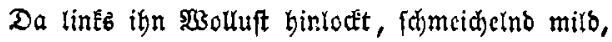

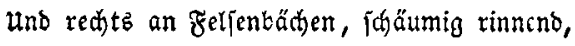
Slym Iugend winkt zum fitein'gen Runpfgefirb, Dod ex, vom jüfen Irugbild ungeblendet, Den mädt'gen Sdritt zur rauben $\mathfrak{B a b n}$ jhon wenoet.

\$(Siebt'z nid)t nod) (Einen, 》 - bad)t' id) - 》ber fo mãble Mit ungebeugtem, fiderm Muth, gleid mir? Der' lieber fid zur Shaar ber Iobten zăhle, 2lह zu ben Rebenben im Rnechtrevier?" und fdau, - wie fonder Bangen, fonder Feble, (id) ein Slnmpier offenbart, alb șier Der Erbenwelt, - bod aud, um fie zu folelten Dan nidyt jie gleidjiegt überizo'jden șelten; -

So bertith bod, , fo milb und cutte cijhicnen, Stans plïglidh neben mir ein fajüner (Siciz. Du fay'f wohl fonft fiton jeine ebten Mienen. Siegt man ing nie an ber Xrena Rreis, 
Sicht mon bod alfnäts isn, wo's gilt, zu bienen, uno jeser ftaunt, wie er zu helfon sveif."

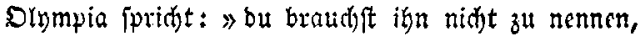
şer wito hidet ba bon Sophron gleth ertennan?

Den Sophron, jebes wüco'gen Shaffens Meifter, Den Sđuth jobrvebes armen biejer Stast, Den Iroft aud mond' verzagenb banger (Bripter! Seil, bas bein Stern ben zHbeführt dir hat!》 Marcellus lädjclt will : $\mathfrak{j} a$, Sophron heißst er, Der weizlid Sinnenbe! bod etwas matt Sag oft fein reizlid Sinnen fid geftalten, unb nidbt bem Sturm bes Rebene Probe balten.

Denn Eaum, bof bom Entrourf, in mir geboren,

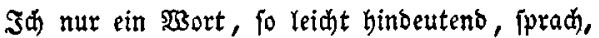

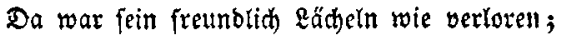

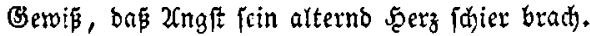
(Fr fallfate: 》weh' bes ungebulb'gen shoren, Der, fheu vor (Frbenj(d)marh, wählt em'ge Sdmadh! D-

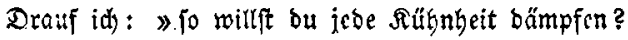
So foll'n auf Eroen nie metre tühn wir tämpfen?* 
228

Da bub cr an mit cinem Sahwall von Sprüasm:

Er fey und id bes Raijers unterthan! -

Rid)tb gleid)' an (Srau'n unb :ßeh' ben (Fibebbrüd)en; -

Stur offen fey vergönnt des Rampfes Babn; -

unb Eurz, aus all' bem zahmen, fänftiglidben

Gerebe ging's bervor, inn fabreclt mein splan.

uno ffrnürt aud Nero fefter ftets bie Retten:

Şels Sophron mag nidht, Eann nid)t, will nidt retton!

Mir ahnt's, ex ift ein Mitglieb jener Sefte, Die farrwädlid Dulben alb bas 5̧öh) fte preift.

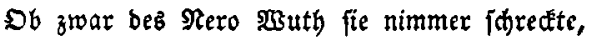

Rebt bod fie ftumm verborgen allermeift,

Itno Seiner nod) aus ibren शännern ftrecte

Den xim zum 20 iberftand mit fühnem Beift.

D6 Taufend rif zum నob ber frevle Sdhlemmer -

Sie ftarben wehrlos, ftill, wie farmade ámmer.

3u biefen Gegnern alter Römertugend,

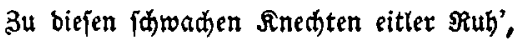

Entfräftenb, ja entherzeno unire Jugenb, -

(ङewi立, ber Sophron, - er gehört bazu!" - 
Da ipridt bie Sdjön' erfareçt: »o, fpäb'nb und lugent

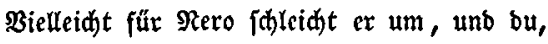
Du wirft fein Spfer! Bon bem brut'gen shäter Fauft ex fih jeinen Frieden als Berrätther!

und bilt eह nut, baß (Finer bod) mus fallen, So fall' ein (Sreis, und nid)t ber junge Seld,

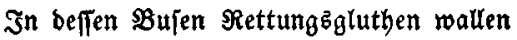

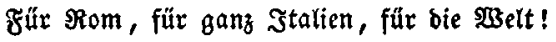
2Bab opferteft ben Einen bu nidht 2Uthen? 》 Marcellub fprid)t: 》 aud mein Sinn war geftellt, NRit בobebgruß ben $\mathfrak{M u n d}$ ihm zu verriegeln, -

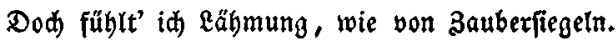

Er: faf' fo ftill mid an, fo mitb ergeben, 2llb ahn' er wohl bie nake, blut'ge sahat, uno fdeibe bod redit heiter ab vom eeben. Die Eippe regt' ex lautlob; -o, er bat Nidtt midh, bod) jeine (sötter, zu vergeben Mir ungeftümen meinen wirben Rath.

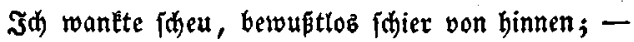

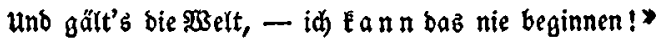


230

- Une [diltit bu nod) ben Sophron, bap ex zage?. So ruft Dlympia, 3orn unb angft im Blice.

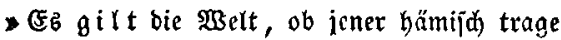
Slad) Rom die Runbe, ob bez Rez̨es Strid um bidh, mein fübnar $2 \mathfrak{a r}$, zuโammenjalage, Birnidhtend fünft'Ber Beiten rubmvoll Btüd. Dem Raifer bebt ber Breib. - Dem Breiz erbebft bu? SBer ift ber Sdrröb're? 2uf! Ridht bix nur lebft bu?

Du lebft ben gropen, rettenden (Şebanten, Die Sertulez bix in bie sruft gelegt.

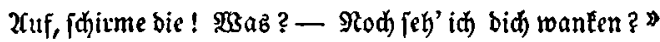
Darcellus ipricht: 》 bu weift, mein Sufen begt Den Scauptitiag unverjegrt in feinen Gdranten. Sur Dradbmen roarf id lơfend aut, geprägt Mit faljd)em Stemper. Mag ber (Sicis benn plaubern! Revo wirb zittern, unb bang fpäheno zaubern.

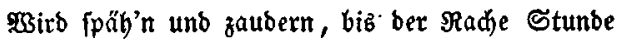
attf ihn hineinbrid)t, (tark in Strafgewalt!" 2ulein Slympia ruft: 》nad) foldyer Sunbe, -

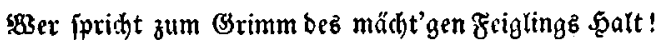


(Erareifen wito ar sid)! und ob bic Runbe Der Freunbe bu brfirmft treu, ftumm und ealt, WBer mag - bu fragteft felbft - ben Schlupftein heben Bum בempelbau? brum: Eeben gilt's an Reben!

Der Strom ber 2 Seltgefdithte - glühend fickst $\mathfrak{e r}$, \$3ie Rabaflut'), und iđhont kcin ebleş Rcis. und jener Galberftorb'ne Men/d - genisft at Daz $\mathfrak{a c b e n}$ noch, umftarrt vom 2uterseiz ? Drum, Seld, zur Ihat! - als ernfter Dpferpriefter Stree"' auf ber Zreiheit Seerd ben müben Sreis. \$ohl mag bann bicies exften Blutens Rinnen

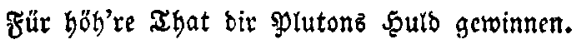

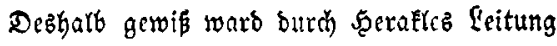
Der alte Furthtiame bir zugejanbt. Ia ja, id fübl's ! Es gilt bie Borbereitung 3um Fettungzjd)lag burd) beine Seldenhand!" Der Süngling ruft: 》 \$̧odherrid) $\mathfrak{B e b e u t u n g}$ Şaft bu tem trilben 2atgenticte entwanbt. (Es gilt! (E)' noth bie Xbenbwoléen thauen, Mup Sophron ober id ben 2rogruno (thauen." - 
232

Fort eilt Marcellub, wunberbar verwilbert;

Dod) wab ithn treibt, nennt er \$Begeiftrungegglut. Seit ign Slympia hat ihm felkft gejairbert arts Dpferpriefter, bürftet er nach $\mathfrak{B l u t}$.

Der (Ev)rfurcht bar, burch Mitleib ungemilbert, פreift ex ben Breifeamoro alß grós und gut, und flúftert leij' in wilden Sangebtönen: 2)

Dod wie ex aud burdjftreift mit eil'gen Runden Nrarț, Gaffen und bie Gärten rings umber, Er kann bie Spur bez Sophron nidt erkunben. So fteht er braufien endlid benn am Meer, Das plöstith aufidăumt, will zum Brimm entbunocn. Eautbrüllend raf't baz zorn'ge Fluthengecr, und wie verwandelt felbft zum Fluthgerinne, Frbebt ber (Srund, und zittert Tyturm und äiune.

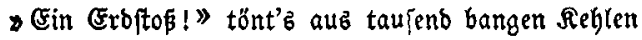
Sn Stabt und Šafen. Und cin ziveiter Stoßs Eridaredt auf'z Reue bie beftürzten Seelen, unb eil'ge fludbt brid)t aus ben ahoren 
KBem nidht zum \&aufen alle Rräfte feblen, Stütmt aus ocs Şaujes wilsberwegtem Siboos, 2ub Mauen fort, bie jüngft nod Sdu entboten, Nun - falfden Freunden gleid - Berjamett'rung oroh'ten.

Sebod) Marcellus ringt bem Strom entgegen Dex F(üd)tenben, uns eilt zur Stabt zurüd Xuf fdrwankenden, gefabrumbrüllten :

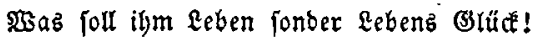
Berwi胍, da nod bebräu'nde Mauern hegen

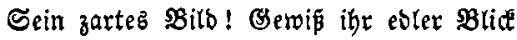
\$ुar fđeu gewandt von roben Fludtgeorängen. (5)' ftürbe fie, alb ba binein fid mengen! Nübn eilt er, um fie rettend fortzutragen Uuf finem ftarken, treu exprüften $\mathcal{X} \mathrm{rm}$,

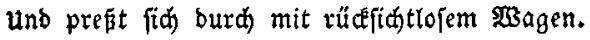
Ihm weidft, fdeu uno brfiegt bex bidte Sdjwarm. Shon fieht ex ihres 5̧aufes (Siebel ragen 2lm leergeworb'nen Marfte + Scoffnungęwarm כritt ex bimein, fhaut fie, all' fein Erlaben! -

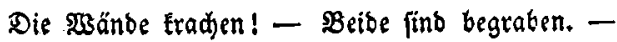


231

3uexf umf hlciert Shnmadit itre Sinne.

Dann Jebca, wic ifm neu ber (ssift erwad)t, Fingt bang' fid), waz frin anbrez Salbft begime. Dab 2(uge forfd)t umfonft. Dir 2logrunbanad)t ફ̧ält 2 flles, wie im bichten Fluthgerinne, Bervoorren, trüb, von Shleiern tief umbad)t. Dod) ale fie rufen fid mit Schnju(t)tbtönen,

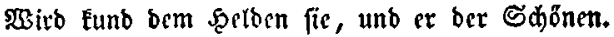

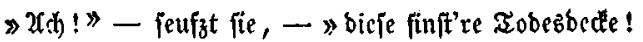
şzie fdrecklid), fo zu fterben, fonber Eidit!》 $\mathbb{E}_{\mathfrak{r}}$ fudst, ob ex kein Fünklein mehr entbcée $\mathfrak{X m}_{\mathrm{m}}$ seerb, und fich', ba glimmt', wie Sternlein brid)t Durd RadtgerwölE, an unzerftötter Ecke

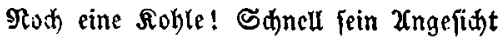
5at hauthend ex barüber hingewenbet. Dlympia fieht'b, von Freube faft geblenbei.

Sie fiegt im sidht erwedter Brht sie 3ugge Des Freunbez, - allerbingz zu balo umbüllt Bom bunketn (Srau'n! - Scooch wie bab aud) trüge, (Er haud)t auf'b গeu', unb weil bic Bruft ibm füllt 


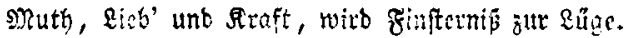
Dic Rlarhcit fiegt! Sic Flamme fteigt, enthullt Xub ounkeln Stoffen mit geroalt'gem Streben, und eine nabe Rerze wabet the leben.

ats nun bie Riebenben fïh wieberifiauten, Marelfus uno Solnmpia, Blid in Blic, $\$$ wie von fel'ger fluth bie 2rugen thauten, Uls gấlt' es fiđf'rse, ungeftörtes Sitüc! Dod) als fie um fid fahen - ba ergrauten

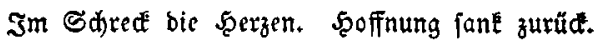

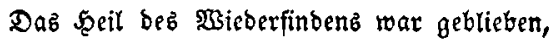
Dod) ftrenge Madt faieb von ber Welt ifr Rieben.

Non fdoner $\mathfrak{B z e l t ! ~ S a r t h c n o p e , ~ b i e ~ B l u ̈ t h e n , ~}$ Die Frühte, die bu ftreutt auf fonn'gen Strans, Die Simmelalidter, bie fo freunolid glüh'ten, In Meer' $\tilde{z}=\mathfrak{X}_{\text {zuren }}$ küblent jel'gen Brano, Die Sđ̆metterlinge, bie wie Rïhter fprüh'ten Sin bura bas ticfumgrünte Mnrtenlanb, Die Riebenden, gewohnt, bas all' zu faduen, Birgt nun des eingeftürzten şaulez (Srauen. 
236

\$rohin Marcellus umbličt, - Irümmermafien Beriperren lidt und (3ang, unb fdier bie $\mathfrak{E}$ uft, und wie mit gierig vorgeriltem Șaffen खुinft feitroărts eine alte urnengruft, Bergeffen feit Jahrbunberten, 一 im graffen Setümmel jezt gepprengt! - Shr Toberbuft Steigt aufrwärtz nach ben Riebenben, wie lockens:

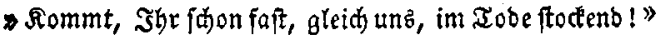

- Es ift vorbei mit Freube, lidit unb aeben!》 So ftöbnt Marcellue, feufat Slympia. Słon brült bie eingefdlof'ne Ruft! Shon weben Die 2 bgrunbanebel, wie fie nimmer ing' (Fin Slid, ber nod) zum lid)t ben Sdwung barf heben, um 5̧erz und Seele fín, ftetz ängft'ger nah', unb biahter ftetb, unb immer wisber biffter; Berlöfłenb finfen fdon ber 2ugen sidgter.

Da plöbliq oröbnt's von Godher wie von झुsttern Deb Frühlingz, wenn in bumpfe \$Binternaht Erneutes Leben :BSolE' uno Stromfluth fdmettern. unb hord! - bet Erofhutt broben wantt und fradt! 
Die Beiben rufen zweiferno igren Göttern; Denn zweifeln fie nid)t an bcr (3)ötter Madit, So wiften fie bod nidt, ob $\mathfrak{x}_{0} \mathrm{~b}$, ob ecten Die unverftand'nen Eenter roolten geben.

Da zum befreiten Erbgruns fay' bie Sonne Sprein mit ihrem reiden Freubenglanz,

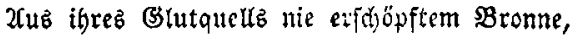
(Banz Siegerin, und Spacht und Sajöngeit ganz. und bod) am Sdlunbe, frablend bell con \$Bonne, Stand yod ein Greiz in weip̈er Locken אranz,

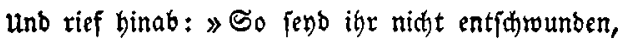
Shr fibönen Bitber? Dank, mein Bott! Befunden!"-

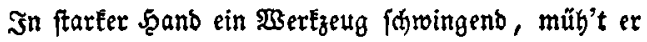
Eid) treulid, ibnen $B a h n$ zu hau'n an's Bon Freub' und Ihateraft uno von SBehmuth glügt et Faft wie ein Şelb, ber vor (Sef(jwabern fid)t, unb gegen eines fieghaft ftarfen $\$$

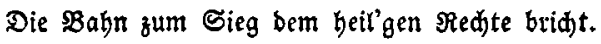
Balb hatten fid bie beiben aufgeidyungen

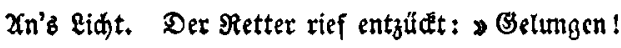


238

(Selungen burth bez göt)iten Retterz Bnate,

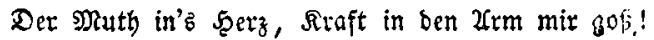

D hilf num, 5̧örhfter, aud zum Sühnungabate, Daz bold befrci'nd unz Gündern alfammt flof, Şilf babin biejen 3roeicn, baßj Fein Styabe

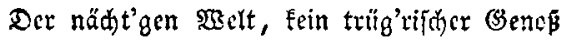

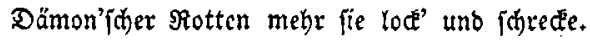

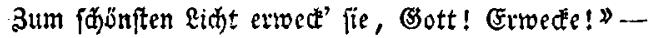

(Fin nod) balb unverftand'nez, fel'gez șallen, BBie wenn fid Meer in Norgenträumen regt,

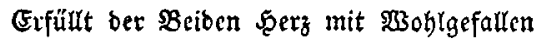
und Demuth, bas fie, aknungeffroh berocgt, Bor itrem greifen Freunde nieberfallen, Die beänbe Ereuzend auf bie $\mathfrak{B r u f t}$ getegt, นno zu ihm aufidjau'n, wie bereu'nbe Sünber, Dod) mehr faft fdou wie reid bejdentte Rinber.

Er: aber leitet ifre bolben ङecten Mit gottbefheib'nem Dffenbarungzwort Dat in, wo's gojung gilt von allen Fehlen, 3:tm überjdrenglid bogen Freubenport. 


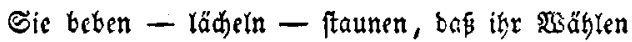

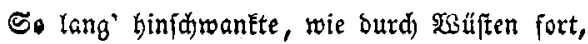
Durd Eabyrintbe, ftets vem sidyt geidededn Derweil jo/nah' fie's rief zu Freub' und Frieben.

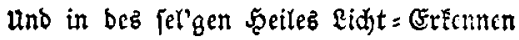
Bargeffen jie's beinahe, ocki igr Stano Zuf Irümmern war! Daß unterirbija) Brennen

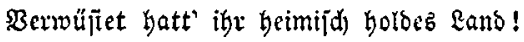

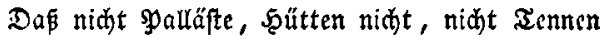
Ringaher nod) ftanden am einft reidam Strand.

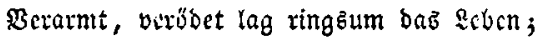
Sie merleten's enblid; - jeģt bod obne Beben.

In Sophron's 2rugen \{ab'n fie, findith fragent. Da fprad ber Şseife: 》folgt mir über's Mecr! Sebt $\mathfrak{s}$ hr bie Maften, hod am ujer rageno? Dort liegt ein Gidifit, aub Inbionz Fłuren tyer. Der Seefturm, $\mathfrak{e}$ an unjern Strans verfidlagent, SEringt (Eut) und mir bie friedlidje (S) Daß wix, in böblften Ricbesid)u, Benommen,

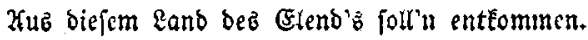




\section{0}

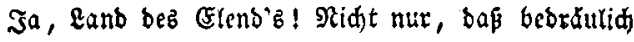
Der \&öwe Rero brüllt vom Sapitol. Berfehmt burd) taujenb Sünoen unb abjicheulich

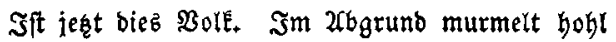

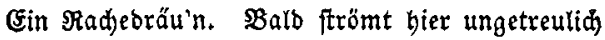

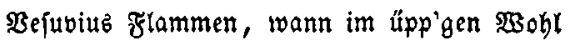
Sid) neu bie Stabt erbaut hat, und in Frieben

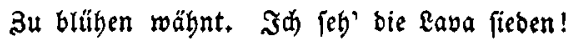

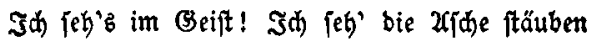
Bom Firmament, begrabend Stabt und $\mathfrak{E} a n b$. Dann liegt im tobesftarrenben Betäluben und in Sabinleben furdtbar feftgebannt Dez Eaum nod) regen Marktes ahun unb Ireiben, und faum wirb Şerkulaneum genannt,

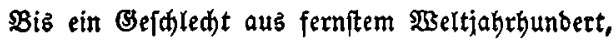
Xufgrabenb ben Ruin, inn fheu bewundert. -

Shon wandte bang' Slympia ihre Iritte. 3ebod) Marcellut ftand und fatwieg und fann; und jeufzend bört' er igre fanfte sitte.

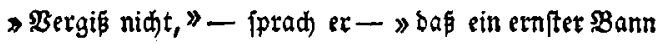


Nidi) fefthält in Gethörter Freunde Mitte.

W3as trüb" auf meinen wilben Rath begann, -

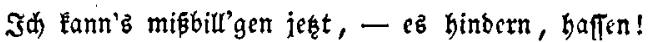

Dod) bie, fo mir vertrauten, nie verlafien." -

Da fprad ber alte, ftarke, fanfte Retter:

》) Getroft, Marcelfus! Deine $\mathfrak{B a h n}$ ift frei,

Du Eennit ben Sain ber unterirb'id)en (sötter,

Durdffinirt bon zorn'ger $\mathfrak{B o ̈ g e l}$ (5raungefdrei!

Den Szain, wo \$hr (Eud) oft als freble Spötter

Beriammelt haút, baß spluton 3euge fey

Dem gleipneriften, treubergép'nem Bunbe; -

Blid" hin! Tief liegt ber Şain im glüb'nben Shlunbe!

Dex rís verlangent $i \hbar n$, als zu erbeben

Die Erbe ankub, tief it fich herab,

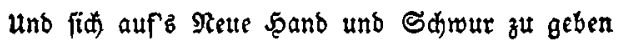

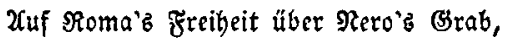

Stand bie Berfanwörungafhaar vercint fo eben.

Man harrte nur nod) Dein, bod Gielt bid. $a b$

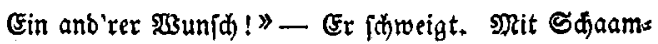
errötyen

Ruft Эenter : 》 Sophron! Sclb! bid) wollt' id töbten! 


\section{2}

Did) fudt' id, in ben abgrund sid) zu betten, unb bald barauf baft bu mid aufgejudt, um aub bem $\mathfrak{Z}$ bgrund [egnend midi zu retten! Xd), bin id nidit zum Segen zut verrud)t? Dod) Sophron fpridt: " getroft! Riegt wer in Retten, Sft Semanb trank, von aller. B̧elt verfludt, $\rightarrow$

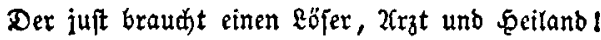
Wer ફ̧ülfe [udit, bem nah't er jeşt wie weiland!

Ję̂t, weiland, und für jel'ge Eroigfeiten! Betroft! $\mathfrak{W u d}$ Eud ift er fdon jeg̨t genah't. Bapt froh burd mid nad) Snbien (Eud) geteiten,

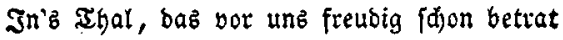
Mand' beil'ger sua erretteter (Grweib'ten! \$3ohlauf! :3ohlauf! Sie bahnten Eud ben Pfab.-

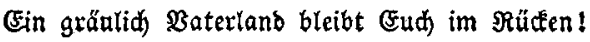

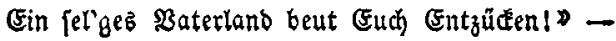

Da folgten fie! Da nahm auf ftille Fluthen Das Meer bie Bark und ifre Eabung an; uns all' bie ungeftümen sisinbe ruh'ten, und jebe gŭnft'ge Strőmung raujift' und tana, 
unb als zulç̧ in jüpen Morgenglutben

Das fđỗue Snbien aufzublüg'n begann

In holber Sraht, im jüfen Düfteregnen, -

Still! - 2uebnlides wirb Senjeit unz begegncn,

\section{Des SEerta Defudi.}

eegendenartig.

Alв मоధ) ber \$err auf Erben litt, zu wecten, bie ba fibliefen, trug oft er weit burdjs sand ben Sdritt, ber IRenja)en Ihun zu prüfen,

3u prüfen, wer ba würoig $\lceil e \mathfrak{l}$. bap ex fein reid erwerbe, unb, wenn ber Erbe Intg vorbei, beb fimmets Aronen erbe.

2d)! Mandjer wies ithm ba bie Thur und weigert Trunt unb Speife; 
244

benn (Söttlid)es böhnt für uno für ber bloben Menidjen $\mathfrak{N 3 e i [ e . ~}$

Dod wieber flupft ber Serr einft an an einer niebern Şütte, unb fieb! es wirb ihm aufgethan unb ruft mit fanfter $B$ itte:

\$ Serein, bu lieber Gaft, Gerein! bein Mantel trieft vom Regen; fomm, trodne bia! es harret bein bes Süttleins ganzer Segen.

3roar Elein unb eng if unjer \$ุaub und wenis, was wir haben; boh) ift'z genug unb reifft rohl aus, Den lieben (Saft zu laben. 》-

Der Feerr tritt ein. Ein greifer paar beut ibm bes (Srupes frieben, zroet fromme 2alt' im Silberhaar, längft von ber șelt geidjieben. 
Sdon mandicr Sommer fam unb wid, jit fie beifammen waren; bod) liebten ihre Sceten fith, wie in betr exften $\mathfrak{J} a b \mathfrak{r n}$.

Sie löjen ikm fein naftę Rleib zu ftärfent lauem $\mathfrak{B a b e}$ und wiffen nidbt, wie nabe heut

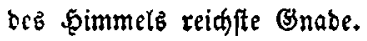

uno raas bie Riebe Riebes hat,

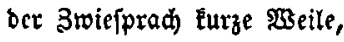
Tranḱ, Speife, פfleg' uno Eagerftatt warb ba bem Şerm zu Theile.

und alb am Morgen er erwadt, Donkt jegnenb or ben 3roeien und (prid)t: 》Sagt, was euch) glüdfich madt; Der Bater wirb's verleiben!"

Da rufan, wie aub Ginem Muns, fie: „ Eineb wünidt bie Secte, 
246

baj Bott in sin' uns fether Stunb'

uns zu ben Seinen zäble. -

unb fdeibend ftreckt bie Şand ex auह :

* $\mathbb{W}_{3}$ obl, alfo foll's gejdichen!

Befegnet fen fo gaftid Şaus, -

$\mathfrak{m}^{B} \mathfrak{g}^{\prime}$ es eư woblergeben!"

und fieb! nod waren nid)t ins Jahr

ber Monde ztwei gegangen,

ba füblten fie fid wunberbar

von Whnungsluft umfangen.

unb frecten auf ihr Rager fid

unb farteten bie Şände

und beteten tief inniglich

um cin gerubig Enbe. -

und ale bes 2 žege am Morgen früh

pin $\mathfrak{1 3 a n b r e r ~ f a m ~ g e z o g e n , ~}$

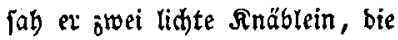

outds sp förtlein eilend flogen. 
und Duft, bes Main Dufte gleid, lodet' einzugehn ben Fremben.

Drin Iagen Beibe ftumm uns bleid in weipen 'robtenfemben.

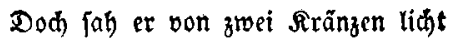
bie greijen Sో)läf umfangen, und auf bem Geitern $2($ ngefid)t ber Süend Rojen prangen.

Denn wer ben 5eern mit Eiebestoort bienteben aufgenommen, ben beipst er bereinft aud bort in feinem şaus willtommen. 


\section{Da leste Eied.}

$\mathfrak{B}$ aItabe.

Mer wanfet bort mit haft'gen Sdyritten 3um Shlop ben öben Gang?

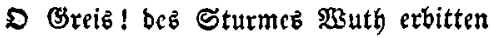

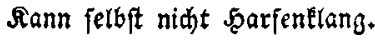

Fr jöneibet Ealt burd alle B̧rieber unb bringt zur warmen sruft. Eriftart ir bort bie ernften sieber, Die bu nod Eaum geroupt? -

Bon Eablgefegten FBinterböben

Siehft bu bez Saloffes Dom, Dod zwifden bir und jenem wehen

Die :sinde auf bem Strom.

\D mag ber raube Winter ftürmen;

Rod) lebt in mir es heí, Da unter jencn bohen İhürmen

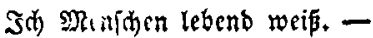


So wie zu mit herütucr bringet

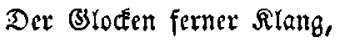

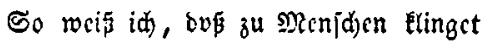

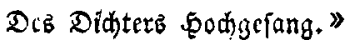

Die Minbe webn in feinen Roden

uno unterm Eije ftögnt's:

Sdon [dwäd)er klingen ifm bie Blodten,

Dod) aนธ Der Tiefe tönt's :

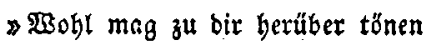

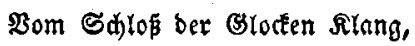

Dodh bie brin robnen, Sänger, böhnen

Des Did)terళ 5̧ంd)gefarg.

TBas willit bu unterm bohen Ihurme,

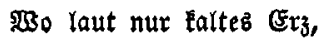

Dod) nie fürs Rieb in heipem Sturme

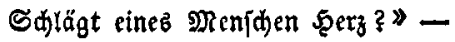

Der \$inb wirb fälter, matt ber Sänger. -

গুas zaubert bort fein $\mathfrak{F} u$ ? 
2.50

Ex bliclet lanB uno immer lănger -

Ea farrt bes Blutes Flup.

Da febt ex an bem Straud fid nieber,

(Beftellt bie Sarf aufz ciz,

Jum leg̨ten kiebe friner kieber

uno fo baginnt ber Greib:

》) WBill mid) ber Sturm und Froft bezningen,

Die ith fo oft befang,

şsill id erft Eübn mit ignen ringen

23or meinem luntergang.

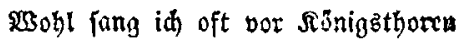

Fữ unbanc ftatt für Golb,

Nun hat's aud bie Satur ver joworen,

Jhr bient' $i d$ treu unb yolb.

Rid)t fing idh beut fum exftenmale

Sor Ealtcm Eib uno Ĕhnce,

In Frübling fand in Berg und $\mathfrak{X}$ bate

उफ) robl nod) fülter eb. 
Die nie mit einem Didter fühlen,

Die nie freut Gangez $\mathfrak{a}$ uft, Die kalten Menjđenherzen fühlen

Dez Săngerz beiße 2 ruft.

Run (d)lage, rauber Sturm, mur immer 2ln's $\mathfrak{g e r z}$ mir, nod ifts beis. Der Menjaden Reben find weit fohlimmer,

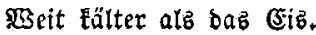

Den heil'gen Sänger foll nid̆t (đ)reden

Der $\mathfrak{R e n j đ e n ~ f a ́ n o ̈ b e r ~ S ̧ o h n ; ~}$

Jd) fang ber (Elemente Sdyreden, Solf fdreden mid ibr Drohn?

Ein e1''ger Frühling btüht dem Diftter, Blau wörbt fichs broben itm;

Er bannt bes Nebelz Truggefirfter uno jebez $\mathfrak{n}$ nethũm.

Drum will bem Norb zum Irog id fingen Bom rarmen Gonnenlint, 
252

unb wie ber Engel Stimmen Elingen,

Bis baf mein Dbem brid)t. 》 -

Es fang bet Breis fo hohe Riebcr

Bon euft und etrgem Rai,

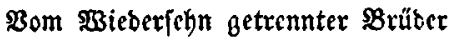
unb Ricb', bie erois treu.

und ale ber Sturmminb nidat mebr wüthet, Sdwisg aud) ber Sänger ftill, Dod facint'z, als ob bie Stirn nod brütet, Gein Muno noh fingen will.

Das Xuge blidet empor zur Sonne, Dod fteigt empor lein Son. 3um erogen Mai, zur ewgen monne æBar Săngers Şeift entflobn.

Frzillibalb aterib. 


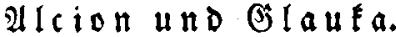

$$
\text { Rad (Songora. }
$$

Jn bie Fluth gejentt bie Reąe, zingebunden jeinen $\mathfrak{R a d}$ en In bem Felfen, beffen Seiten Meerezwellen leiz beroegen,

Rlagt ber formacistende 2atcion, Dás niđt fommt die fojöne Shaufa, Sie, ber Stolz ber banzen Gegent, zaler siid)er Riebebflamme.

Mit ben 2 ugen rad) igr fpähenb Qäft er laut bie Stimm' eridallen: 》 Blaufa, ruft er, was verweilft bu? 23arum läßt bu auf bid harren?"

\5̧aft bu etra Reu' empfunben, Daf bu geftern mir verfprabeft, 
3u mir an ben Strand zu Eommen, (Eh' bie Somne aufgegangen? "

\D bu Faljobe, wenn baz Deiner ๔dwüre nidht willit balten, WBerben meine ahtänen|tröme

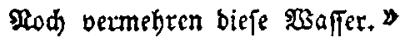

2 Meine GSTauka, bift bu ferme? Doer williz bu an den sualen, Xn ben Xertgften mcines ફ̧erzents als ein Sdabenfroh big Iaben?

D Sit bem [0, verzcif' ith getne 2ul bein 38 gect, wirft bu fagen, \$3as id) Sutca voer Sd)limmes Ban bir zu exworten babe,"

- Dod mir 2rmen, wie viel Beiden Deuten an ber areue $\mathfrak{B z a n f e n !}$ Stärter weben jeğt bie Şöher heben fid bie 
Die Delphine, fie exidcined Sdywimmend auf bem \$Bellenrante, ungewitter broht bem seere:

Beh mix, Blaufa'z Iretle wonfet!》

Run erfdien Die fdöne Rymphe, Raciten Fufeb, am Seftabe, um bie Reuren zu befratuen, Ju bet'bern igre șamen.

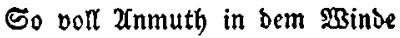
Shre blonben Rocken ffattern, Das mebr, als bie 2angeln Fifdee, Shre saare 5ecrzen fangen.

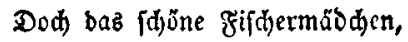
Die vernommen feine. Alagen, War bavon fo [dled)t erbauet, Daß fie Uebel ärger madte.

Xlo fie merkte, wie XIcion grach ibr fdaute mit Berlangen 
236

unb iffr alle fijothe wegfing

Nit ben Reujen unb ben Şamen;

Bab fie tahend ihm zur Zntwort:

》 $\mathfrak{L}_{\beta}$ ez gut jeyn, nicht mebr Elage,

Danfe mir mein lang Berwocilen,

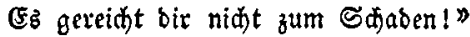

unt fo fehrte fie, ifm zưment, gałă binlaufend lângz bem Stranor,

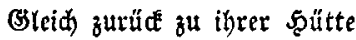
unb ber Fifder in ben Rathen.

Beauregaro gandin.

Der Sisnig uno der page.

Die Sirone auf bem Şaupte, bas בepter in ter Şans, Jnmitten feiner Mamen ein greijer א̋̈nig fanb.

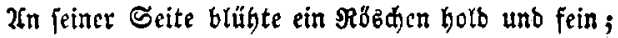

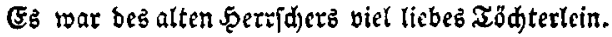




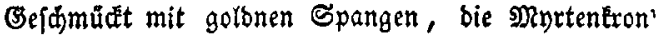
im Şaar,

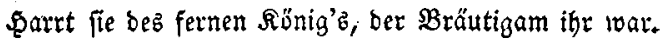
Dod) adi ! fein füß (Evbangen crfillte ifse Sruft,

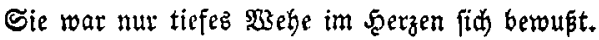

3um ¡̆blanken GolEnaben, im Dienft ber Rönigit, Sah fie mit feuchten 2lugen zumeilen fohühtern bin;

Shyn batte fie extobren, ex war ihr bödffes (slüd unb er gab gern bas Reben um ihren holben Blid.

Da fळmettert's wie Zrompeten, ein Serolo nahet fí, Reigt tief baz șaupt bem Rönig, beginnet feicrlid):

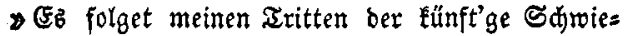
gerfohn,

3u bieten beiner sod)ter fein Serz unb feinen Ifron. 》

Der Aonig ruft ben Şagen, ber sage fliegt herbei. -

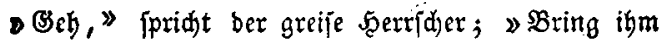
Dab SPfand ber ßreu

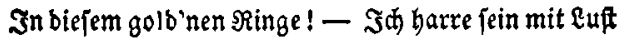
uns freue mid) zu orfiden inn an bie Baterbruft. 


\section{8}

Und als ber sage ferne, fturzt mit beroegtem Sinn Die bolbe Rönigztodter auf inre sniee bin:

- Dein $\mathfrak{B a t e r}$ und mein $\mathfrak{R}$ önig, ruft börbar loum ibr Muno,

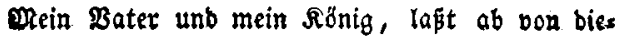
fem $\mathfrak{B u n d}$ ! -

Id) tann ben Mann nidt lieben, ber fremo unb uns belannt,

Wix bieten läpt bie Srone, fein Serz unb feine Đanb! Die Tiefen meiner Seele füllt längft ein ander Billo,

$D$ Bater! babt Erbarmen, jeyb gräbig mif unb mild, 》

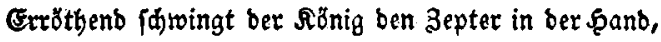
und fprid)t zu feiner Bodter, die Blide abgerwand: - Drein lung bier,

und wait id es gegeben, ift es aud beilig mir. Du bift beš eblen Rönige längfi anertannte Braut, grzirft morgen bor'm 2ritare ihm ewig angetraut l - 
Berbollt finb laum bie \$3orte, fteht botb ges idmüclet \{ðon,

Bam Serold eingeführet, ber Grembe vor bem Thron.

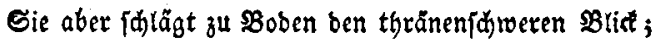
stein jüpes 2rgnen tünbet ige namenlojez Brüd.

Jm armen ફerzen wathet ein ungemesner Şarm, unb bleid und lebloz jinket fie in ber grauen xrm.

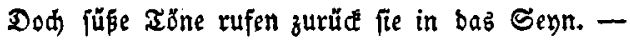
Entflogen ift ber Summer, entflohen 2ungit und spein; In ifrer Seite Enieet ber Edoelfnabe bolb, fuf feinem \$aupt bie אrone von Eberftein und Golb.

" Du innig heiß Befiebte, Und brü dite bie Erftaunte an bie berwegte $\mathfrak{B r u f t ~ - ~}$

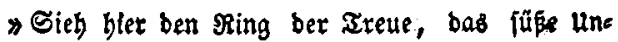
texpfanb,

Den mir alb Riebeszeidjen burd) mid bein Pater fanbt'. 
260

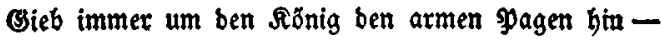

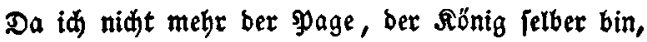

Den R̊nig wirft bu lieben, ba bu bem syagen treu,

Der sage treu bir bienen, ob ex aud Rónig \{ey! ty

Ibeopbania.

\section{Des Minnefăngers . Betmådtniß̄.}

- Walther yon ber Bogelweibe

Nennt midh alten Mann bie $\mathfrak{B e l t ,}$ unb ein :Betbplase, wann id idetbe,

Gey ben $\mathfrak{B o ̈ g e t e i n ~ b e f t e l l t , ~ " ) ~}$

- Meinen Leidnam zu bebecten,

Wählet einen fladjen Stein,

und vier \$őhlen an ben Eefen

Meifelt tief und fauber ein. 
ฟ Füllet täglid biefe Becher Mit bes $\mathfrak{B a d h e z}$ reiner Fluth Für bie hädft befdeionen Bెedher,

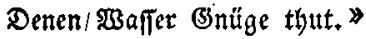

\$) Utnd auf meineb Grabfteing Mitte

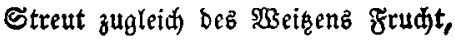
Dap bie Sdian zu (Gaft fid) bitte,

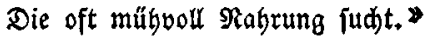

2ftริ ber gute Minnefänger Sein sermäd)tnis fo gemad)t, Stunbet' ihm ber aob nidjt länger

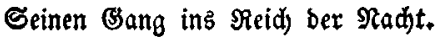

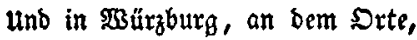
$\$ 280$ er bauf'te lange 3eit, Warb ihm yor bez פünfters Pforte Seine Rubeftatt geweint. Эhre grünen Urme ftredten Sobe sinden brüber bin, $\mathfrak{u n b}$ bie $\mathfrak{B o p g e l e i n ~ e n t b e d t e n ~}$ salo ben reidjen frudjtaesinn. 
262

Freubig flogen fie bernieber, Eabten fich mit Speip und Sranl, Shwirten auf bie Băume wieber, Sangen bort bem Seber Dant.

Dod erlebte bieß Eciber nur ein nahes $\mathfrak{s} a \mathfrak{b}$, Db'z zu ewigem Sebăd (3leid) unlăngft geftiftet war. Denn ber Ehorbert'n bobfes Geigen unterbrad) ber Spenbe Rauf, und fie fammelten ben 13 eişen fin fich felbft zu হuben auf. Xud) bas şaffer liep man feblen;

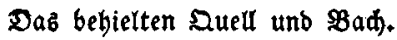
Sene weingewohnten Reblen Gebnten nimmer fith barnad. 


\section{$\mathfrak{A} \mathfrak{i} \in \mathfrak{g} \in \mathfrak{t}: \mathfrak{f} \mathfrak{u} \in$ \\ Ballabe.")}

Die Fcinbe flieh'n, unb mit verweg'nem Tagen

Stürmt ifnen nah ber Reiter fühner Sdwarm

Sdjon viele liegen blutig und exjhlagen,

Dod) nod) nidit ruht ber fiegoeroobnte $2(r m$, and hingeftredt von wohlgeführten Streiden Dup mander nod) ber Fr(iehenden erbleiden.

Boran bem 3ug' feht ibr ben Führer fliegen;

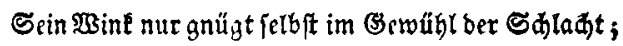
Mre mit ihm ift, will fallen ober fiegen,

Gür ihn wirb gern bas Eeben bargebraht; Denn nidt ben Erften bloz zu אampf und Den Bater hat bie Sdaar in igm gefunden.

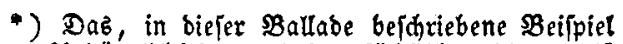

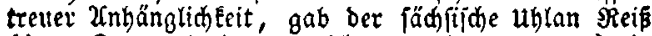
(iekst Rorporal im 1. leidten Reiter = Regte.) in bem (3efect)te bei Ialaticze, am 1. Robbr. 1812, in weldem ber Major bon Sénbliz blieb. - Z Felozinge ber Sahjen 26. Seite 82. 
264

Shn rei巨st in ¡dnellem rositgeftredtem $\mathfrak{L a u f e}$

Sein gutes Rop jekt immer weiter fort Saum folgen tann ber Geinen treuer Şaufe,

Denn not) verweilen Feinbe bier und bort, und faft fhon iff er wieber mitten innen, Den Sieg zum zrocitenmale zu gersinnen.

Da menbet fid), bem brốnnenden Sefedte

Scalb fhon enteilt auf fludigerohntem PFerb', Der Feinde Einer, unb, bie mübe Rechte

Der Ranze joon verfagend und bem Sdroerdt,

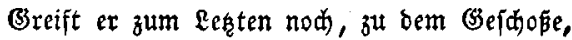

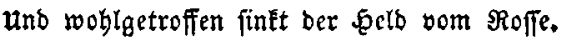

Bellagt inn nidit, bas et fo frufh gefallen, Redyt in ber Mitte jeiner Selbenbahn! Benetbet ihn! wer fäbe von uns 2 ulen

Nicht aljo gern bie Tobezfunde nab'n? unb hat er nidit, inbem er früh gefhieden, Manch bitt'res Loos, baš unz betraf, gemieben? -

Jubeffen fommen, ibm vereint, oft fiegen, Die wate'ren Reiter ibrem Fübrer nad - 
Sie fegen inn mit off'ner

Dem jđon bas Zug' im Tobestampfe brach,

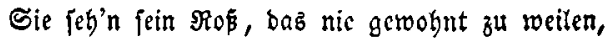
3um Feinde berrentos binübereiten.

Da fast, zumar mit Irauer und Entieğen,

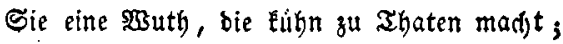
Die jprengen bor, bem Feinbe nachzulez̧en, Der bes gełung'nen Sduffes jubetno ladt, Die theilen fich, wo rings nod Feinde Eämpfen,

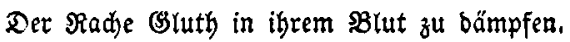

und Einer nur, ber ftets yor Bielen thetuer Dem 5eelben war ob jeiner Tapferkeit, sezähmt bez Muthez ungeftümez zeuer, Den glükenbheifen Drang nach blut'gem Streit';

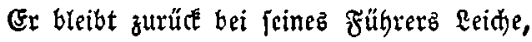
Dap ex ben Iescten Dienft ifm nod) erzeige.

》Soll id ihn bier fo unbejdirmet laffen,

T3ర bald vielleidyt bes Feinbes roher $2 \mathrm{crm}$ Mit fanober Bier ifn raubend wirb exfaffen, Nod) zu verboppern unjern tiefen Şarm? 
Nein! măst ${ }^{2}$ id brob mit taujen geinben fteriten,

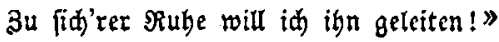

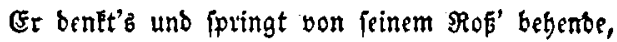
Belabet fid mit ber getiebten $\mathfrak{E} a$ ft, Eో)lingt um ben 5̧alb bie Galberftorb'nen Şănde, uno trägt - ob fraftlos er erliege faft Den Ighu'ren aus Dein wirren Rampfgetwäble Dit treuer Riebe idmerzlithem (Sefüble.

Die Feinbe num, bie ihm vorüberjagen,

Benutęen nod) Den 2 Uugenbliđ ber Fludt; Der müht fid), mit ber fanze ign zut ihlagen,

T3enn Jenez Stahl ihn zu burdbohren fudt; unb ferbft bie אugeln, bie von fernher pjeifen,

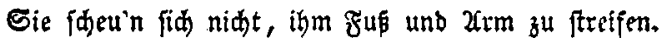

\$Ström' bin, mein Blut! ftröm' bin aub allen 2bern!

Waa gilt mein $\mathfrak{E} e b e n$, ba ber Führer ftarb? 25ohl mödt' id zürnend mit bem Sdidfal babern,

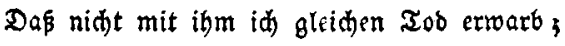
Sour bes id jekt, ad)! jeste nod nidt erbleide,

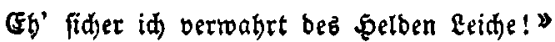


So rufend trägt er, mitten burch Befahten, Stywerathment fie zu wohlverboig'nem Sort, und legt, vom feinbe nidit mehr zu getwahren,

Sie nieber janft mit biefem Trauerwort: - Sier ruhe bu bis wir ben Feind gejdlagen unb bid) zu beiner ew'gen Ruke tragen!*

Drauf fpringt ex fort, ob bie erfdlafften Gffeber

Sum Dienfte nidft inebr willig und bereit Wobl fdrongt ex oft, bod ex ermannt fid wieber -

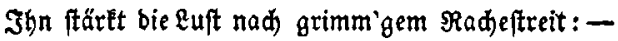
Des Führers \$Reippiel bis zur lck̨ten Stunbe Eebrt' ibn, wie fin ber wabre Muth befunde.

und feht! von einem Segner, ber eriflagen

Im Boben liegt, fängt ex bas flüd)t'ge Roß; Er wirft fid brauf, und nad mit witbem Jagen

Gtürmt er ber Feinbe weit entflob'nem $\mathfrak{x}$ rop'; \$od fidringt ben Speer bie feftgeballte Redte and mitten if er wieber im (sefedte.

\$3obl Eann ber Sänger eud nidt offenbaten,

Wer nod) als blut'ges בobtcropfer fiel 
368

Bon feinem 24rme aus deb Feinbes Shaaren,

Dod) Rad) war und blieb fein fteteb $3 \mathfrak{Z i e l}_{3}$

und wie ber fährer ehrenvoll zu leben, 3u fterben [o, fein unverrü tes Streben.

Ebuarb yon

Der Sdáfer und Die Sdáferin.

Der Sduăfer fás an einem $\mathfrak{B a d}$

unb jah unb jang hinein,

fang fort unb fort fein 203 eh und 2 (d),

uno jeufzte leis barein:

\ Du $\mathfrak{B a ̈ d}$ )cin lieb, bu Båd)lein Gell,

nimm freunblid, was id gab,

unb trag' mein Eieb, o trag' es fdinell

zu ifhr, zu ihr binab!

und nimm nur aud mein Bilt mit fort unb fhene" 


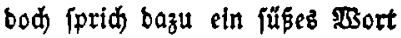

von ihrem Sdüfer hier. 》-

Ş3eit unten

uno /âh ins $\mathfrak{B a ̈ d}$ Yrin licht;

bod) jah fein siltonip fie barm

und bört nid)t, twaz $\mathfrak{e}$ s pritidt. -

unb wieber Eam zum saa baber

ber Sdjäfer treu uno gut,

nid)t $\mathfrak{B a ̈ h l l e i n ~ j e k g t , ~ - ~ e i n ~ w i l b e s ~ M e e r , ~}$

Godjdwellend, Fluth an' Fluth.

》 So techt, mein $s_{a h}$, mein trauter Freuno!

weipt bu, was 5erzleib ift,

bas bu, weil jest mein 2fuge weint,

gum Strom geworben bift?

unb meinft bu's wabx, fo nimm min auf

in beinen reiden Sdyooz, unb geht zu ihr bein idneller Eauf, leg' mid bei ifix ins Proos! ! -

So baudit ex aus mit tungptum zum leşten Mal pein 
und mertet nidht, bas binter ibm fein laujăeno Mägdlein fteh'.

Das hônt nun beffer, was er fprid̆t, unb fieht jein $B$ ilb nun autb; unb ruft mit Sculb im 2(ngefidt: - WBie ? wär' bas Sdåferbraud ?

Bingft bu hinab, mûst' id ja nad, unb 'cbt' ad)! gerne bod! D fieh mid) an, bie nidhtz verbrad, and jage, willft bu nod?

DDid, ruft er, will id, Nägolein tmut, nur bid ! " und fpringt empor, unb fieh! ein Doppelbilonis fdaut nun aus bex Fluth empor. -

unb wieber tam zum SBad unb faß am Ranb ein liebenb saar a! wie ba rann burh) liateb Gras basิ Bä́d) lein ftilu unb klar! 
$\mathfrak{D} \mathfrak{r} \quad \mathfrak{S} \mathfrak{d} \mathfrak{w} \mathfrak{a}$.

Cinfam ${ }^{\circ} 0 g$, burd) gruึne frluten biejez See's ein äbler Sdroan, unter Mittagefonnengluten, unter Mond = und Sternenbahn.

Eirten, ihre Seerden leitend, Gaben oft ben Sang gebört, Den 2fyoll, bie 3ukunft beutend, feinem sicbling felbjt gelebrt. ${ }^{*}$ )

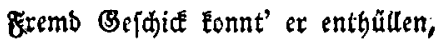
Den fein rignes Shidéal trügt; frembem Sang laujht er im. Stillen, bem Eein eigner Sang genügt.

Jn ben weiten Flutenreidjen

ftrebt er zur Geliebten bin,

*) Der Sdwan ift belanntlid ber Riebling Apolls,

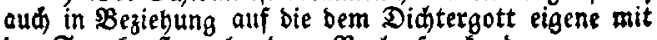
ber Sangfunft verbundene Borberfagetunde. 
endidid bofft er zu erweidien barten Sinn burch treuen Sinn.

Will in Scoffnung nicht ermüben, beren Strahl ben 2 rmen trog,

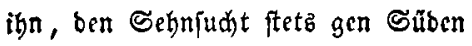
in bie [döne Sceimath zog.

Jahre Eamen, Jahre fhivanben;

Blütben, 2Uebren, Frudbt und (Eib, fahen ing in liebebbanden, boten nie ber \&iebe spreib.

ad) fie warb bez Todez Beute, fie, um beren Sonft ex roarb! Rieben fonnt' ex keine 3roeite, fang bas Sd)wannlies und farb. Arthur oom Rorbftern. 


\section{Die rotbe Sdleife.}

( Nad einer wabren $B$ egebengeit.)

" NBie glifdelid Du bift, Erwine!" Gatte bie liebs: lidi) June zu mir gejagt, alb fie zum sall midh fo

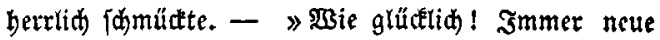
Bergnügungen warten Deiner; bon einer Ruftbarfeit zur anbern wirft Du gleidjam gebrängt, inbes wir Xnbern hier fisen, unb bor Eangeroeile nidht wiffen, wie wir bie 3eit verbringen follen!?

Sie batte Recht, bie gute anne, uno id badite mandbmal, wenn mid bie rafhen Fühle ber şrin= zeffin aus ber glänzenthen Berjammilung zum bril lanteften sBalle wie im Fluge babin trugen: $\mathfrak{s}$ b bin bod) gewif eins bon Fortunens Rieblinge = Rinbern; immer beiter, immer frob, immer genupreid) entrollt mir ein $\mathfrak{s a g}$ nath bem andern - und bief ftetb obne

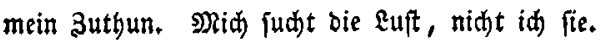




\section{4}

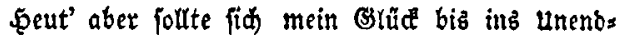
lidje erböben. Mäbe von vielem Ianzen, ermattet von Şören unb Seken, ben אopf yol bunter (Saule: leien, wollt' id eben aus meinem Lioner Balfeletoe ins

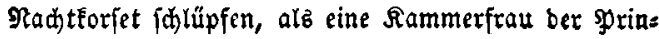

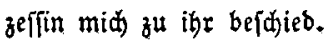

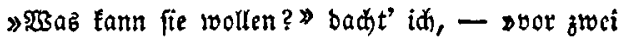
Minuten exft vertieß id fie, jest Morgens brei uhr - mäbe vom $\mathfrak{B a l l}$, fłon im batben Nadtge= wand? "

Inder , id mufte bem Befehl getordien, und fo ftanb id), id)laftrunfen, mit faft zufallenben 2fugen, vor ber Prinzefin, in beren Şaule id) bie Stelle

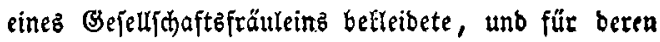
Riebling idh nebenbei galt.

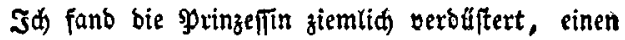
Brief in ber Şallo, auf bem Sopha fitzent. 》Ere

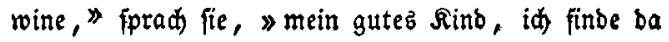
eben sin Sdareiben von ber regierenden Fürftin bon E., mciner febr theuern Freundin, beffen sngalt Did) betrifft. 
》E\& warb nämlid Dein Bater, 》 fuht bie Fürts ftin langjam fort, weil jie fah, dap idy gar nidt neugierig warb, 》vor einiger 3eit von ber Frau Füre ftin berufen, einige Monate biefes Sommerz auf igrem Euftidloffe Mon Repos zu verleben, um cin fehr widtiges Befdäft zu beendigen. Die Fürftin wünjute, Dein Bater mödte Did für biefe 3eit ihrer Iodtex Scortenfie zur Befelly aft mitbringen, umb Dein Bater wollte nidts entidbeiben, weil $\mathfrak{D u}$ in meinem 5̧aule feift. Run rendet bie Fürrftin fid) an mid), uno id lebe mid faft. genötbigt, ben Founju meiner Freunbin zu erfüllen, fo ungern id Did) miffen merbe." Jho trat eine ahräne ins Huge, uns fie umarmte midh mit vieler Innigkeit.

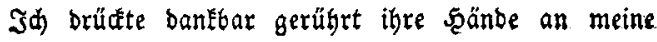
sippen, und fie fprad weiter:

\) get'ft einer Yangen Reihe von Bergnügune gen entgegen, mein Rinb, taulenbe von Freuben werben Did umidrweben, bas $\mathfrak{L}_{\text {eben }}$ wirb fid Dir von feinex jäönfen, lachenbften Seite entgegenftels len - Du wirft Befannt;daften madien, Fteunbs fłaften anfnüpfen, man wirb Did lieben, man wixt 
276

Dir \{hmeideln. Du betrittf am Sofe ber prad)t= diebenben Fürftin einen äuferił gefăbrlid)en, (đ)lüpfri= gen Boben - unb Erwine - Du ftehit allein benn Deines Baters $B_{e}[$ daffe werben ihm nidht er= lauben, Did fehr zu beachten. Berfprid) mir baber, Du wolleft Deiner Eebbaftigkeit Sdhranten fezen, Du wolleft Did nid)t jebem Bergnügen, bas fidh Dir barbietet, überlaffen, Su wolleft Did oft an mid erinnern, unb in ber gröpten \&uft immer bebenten, wie oft ein einziger Moment unjer ganzes Rebens= glüd ftören Eann!" - -

Xaht rage barauf pap ifh mit meinem $\mathfrak{B a t e r}$ im

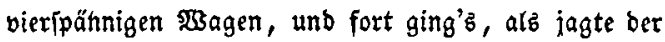

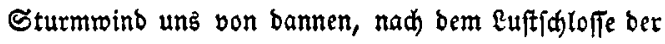
Fürftin.

Ja) blinzte bie 2 ugen $z u$, bamit id in meinen Gebanfen nicht geftort woübe, unb unterorücte nur mit Mübe mein inneres $\mathfrak{a} a$ den. $\mathfrak{J} a, j a$, bie gute anne hat redht, badt' id, id werbe zu allen $\mathfrak{B e r}=$ gnügungen oroentlich gcbrängt. Trie viele mögen mid jest beneiben! - 2 d) ez ift bod fab̉n auf ber

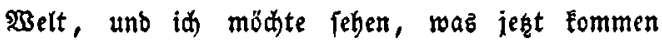


Eönnte, bas meine frreube ftörte! - ఎ $\mathfrak{D}$ roie glüd: lich finb Sie, mein Fräulein 》 - fagte jeufzend auf ber leşten Station bez গpoftmeifters bübjhe Jodjter $\mathfrak{z}^{\mathrm{u}}$ mix - 》wie beneibenzwerth! - ber 2utfenthalt Der Fürftin \&. ift ein Yarabirz, fie jelbft ein (Engel - unb bie Bergnügungen und aerftreunngen find bei ihr ohn' Ende." -

Эd) Idfte bie Bänber meines Staubmantele auf, id) lüftete ihn mehr unb mehr, alb fey ber leidjte Iaffet mir zu (d)wer; eigentlid) aber war bies nux

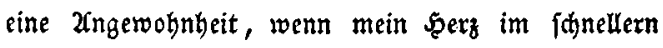
rakte julug - unb eben jest hüpfie ez in \$pejtif= fimo, benn wir näberten unb bem vorerwägnten $\mathfrak{a} a=$ rabieje. Rod) cinmal fnallte ber goptitlion in bie Yfferbe; wir bonnerten über bie 3ugbrüdle, uno waren im $\mathbb{S}$ blopgofe.

Biele reid gekleibete Bebienten umringten umíern 23agen, aber in ihren Mienen lag Irauer. Dex Şaußjofmeifter führte unz in bie für unz beftimm= ten Simmer; auf feinem bleiden Befidit mablte fid ber Jammer jo beutlich, baf id befürdtete, er roütbe $\mathfrak{z}^{4}$ weinen anfangen. $23 \mathfrak{a z}$ ift baz? frug id mid 
278

leife - roitb bies Parables yon Sammer = Geftalten berobnt?

Mein Bater fragte nadi ber Fftritin, um ifs fos gleid) Feine (Ebrfurd)t zu beweifen; ber 5̧aub̆ofmets fter antwortete: 》Durdflaudt find perreift, auf einige

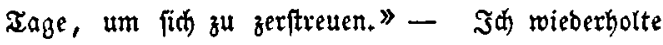

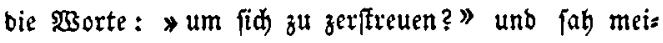
nen $\mathfrak{B a t e r}$ fragenb an.

(Enblith liez biefer fith bei \$pringes Sortenfien melben - und warb angenommen, exiditen aber bald barauf wieber mit fehr vcroüftertem (seficht. Dir warb bange; id warf mid in feine 2rinte. - Eieber Bater," rief id), " aud Sie? - TBas it eह benn, bas fo fdgredend mix heut' vor bie 2luger tritt? id Ieje Irauer auf jebem (3)fitht, - tweld) ein $\mathfrak{u n g l u ̈ c}$ ift geideben? 》-

Mein Bater nahm midh bei ber Scano, führte mid) aber ben Sdlopbof, einem Gellerleudteten Saale zu, unb ragte fehr ernft: 叉(Erwine, ith fübre Dith an ben Sarg eineb felyr f(t)önen, fehr unglüdtid en $\mathfrak{M a ̈ b =}$ Фenb. Drüde ifr Bilb feft in Deine Seele. Mot:

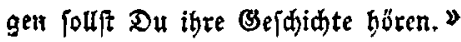


Эa) (d)auberte eit wenig zujammen; bies hatte id nidit erwartet; id bielt meines Baterb 2 rem feft, uns trat in ben Saal.

Im Sdrge, von mattem Serzenichein umbämmert, lag — im weiken Fleibe, eine bunketrothe Sdleife

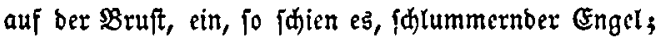
bie forwarzen Lodéen wallten bis zu ben Inieen herab; bie linke Şanb hielt ein fleincs אruzifix, indeß bie Redite auf bem nidft mebr fdhlagenden Werzen ruhte. Das 2 uge, obglcich gefdloffen, verrieth bennod) ble Sduönheit, bie re lebend von fith geftrahlt hatte; bie bohe, blenbend weipe Stirn beutete auf to viel Bsrift, bab ganze Befidt auf fo reidyen Riebreiz -

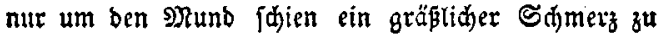
\{drocben.

(E⿱ waren viele Menichen im Saal, größtentheils Eansbole, und id börte bie \$3orte leiß binter mir fpred)en: 》 Sis gab fid ben Iob fetbft, bie blute

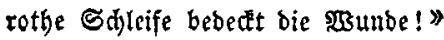

Эa) bat meinen Bater, mit mir ben Szal zu verlaffen; meine Inice bebten, id zitterto unb folsace

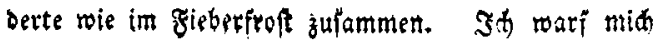


auf's Bett, alb id auf mein Bimmer tam; id fühlte mich frank - und war es wirflid; fo jegr hatte ber Znblick, fo jebr jene ssorte auf midh gewirft.

2fts idh am anbern Morgen aus einem unxubigen

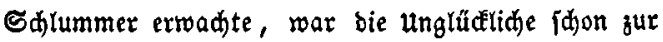
Erbe beftattet, unb ber Secretair ber Fürftin theilte

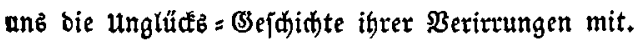

Dubal, ber cinzige Sokn ziemlich rohlyabenter Jeltern zu פaria, Yernte in Borbeaux bei bem Batcr Eucienz, einem frkr in Ruf ftehenden Tapezierer. Duval war ady ter ber leétern ftarb. Der $\mathfrak{B a t e r}$, ein wenig ges bilbeter Mann, veridmerzte ben Bertuft jeiner (Sat: tin nux zu balb; nidjt fo Eucie, bie bie Rutter über Julez geliebt hatte. Duval fah bes Rinbes tiefen Sd)merz, und fein Serz neigte fíl mit zärtlidjer

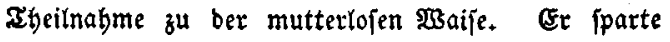
fein Sonntagb̈gets, um ifr, Durd Eleine Spietereion,

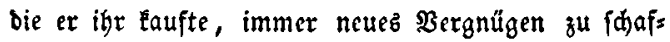
fen; er ging nidht metre wie fonft mit feinen אams meraben \{pazieren, Fonbern (d) lic) ber guten Iodter auf ben Sirdyof nad, unb ruste burd) mandertei 


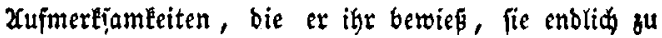
zerftretten, zu berubigen.

Da beirathete Eucienb Bater toicber, und mit bent Gintritte ber neten Mutter in inres Mannes 5̧aus, ging $\mathfrak{R}_{\text {ucienz }}$ Reiben an. Die junge Frau gewahrte

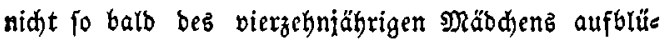
benbe Shänkeit, alz biejes für fie ein aicl bez bit=

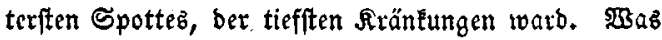
aud) Rucie that, bev Stiefmutter siebe zu getwinnen, eb war umjonft, und alb endlid nad einem Jahe Jene ferbft ein Iobdterden gebar, ba erftieg ihr $\mathfrak{5 a p}$ Begen elucien ben bödiften $\mathfrak{G r a b}$.

Der $\mathfrak{B a t e r}$, beffen erfte Jrau bie Sanftmuth ferbft betwejen war, unb ber bei bem furienartigen 2 sefen ber zweiten felbft böjes Spiel hatte, neigte fich nad und nad) zu feinem gemifgandelten exften Sinbe. Run fann bie junge Frau lange vergebenz, wie fie Eucien, ben umithurbigen Begenftand ihrer 3 suth, entfernen könnte; endtid watb ein Mittel gefunben; Rucie follte ins flofter.

So fefre ber Bater anfänglid ben গ̧lan verwarf, fo jab ex am Enbe boch ein, bas bieß ber einzige 
282

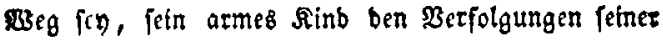
Frau zu entziehen; ez warb untwiberruflich bejhlof= Ten, Rucie jolle ben Shleier nehmen.

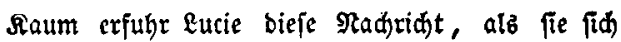
bor Duval auf bie Snize warf, unb ihn bei Bott uno allen 5eiligen befdwor, fie zu retten.

Duval trocénete igr bie Ihränen von ben jä̆nen

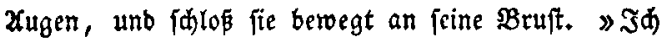
rette Didh, \&ucie!" rief er - fer rubig; ftelle Did, alb ergäbit $\mathfrak{D u}$ bidh in Dein Sdjicéal. So wahr Gott mix einft helfen möge, fo gerwis werbe id Dir helfen! 》

Eucie brüđte banfbar gerưht jeine şănbe an îne Bruft. 》D Du unauşpredtich guter, $\mathfrak{D u}$ getiebter Dubal!" rief fíe ihm zärtlich zu. Bald fam ber Jag ber beftimmten Woreife, und am Borabend rief ber Bater \&ucien zu jith. «Du fiteibeft von uns, " iprad) er, " und es ift Berubigung für mid, bas id

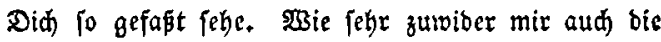

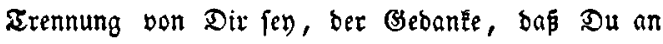
bem Srte, wohin id Did bringen werbe, yor allen Berfolgungen gefidhert bift, mus mid barüber trofften. 
(Slaube mix, ss mustc fo icnn, unb baft Du aud

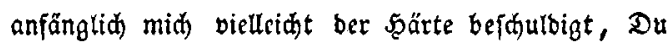
wirft bald einjegen lernen, bas id Dir bas befte Ihcil erroult Gabe, 》

Eutie war gerührt; fie badfte baran, whe vich cher fie fid) yon bem $\mathfrak{B a t c r}$ trennen woütoe, als cr meinte.

2uf igrem Stübden marf fie fth vor ihren Sdus beiligen auf bie Siniee uno betete laut: 》o jhühe Dou midh, rette Dou midh, wenn bes Zreundes ફ̧ülfe zu farwad) feyn follte!》 ba flopfte es leife anz Fene fter; baftig griff eucie nad) bem Elcinen gaädd)en Wäßjhe, bas fie mitzunebmen gebadtem, uno idhlid leije binab in ben Barten.

Die Nacht war fehr finfter. Dumal falok bas zitternbe Mäbden in jeine Urme; „ $\mathfrak{E e b}^{\prime}$ wohl, \&ucie! " ftammelte er - 》mein Freunb Georg bringt Difi) nad) \$aris zu meinen 2cltern; jobalo meine lehrzeit vorüber ift, fehe id Didh wieber." Beorg yob bas jdwankente Mäbden in ben bercit ftehens ben Wagen und rollte mit ihr bayon. 


\section{4}

Die Seftürzung ber Weltern beim Sermiffen ber

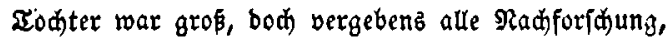
allex Berbadt. Eucienz eingiger freuno war Dou= val, unb bicjer, bas wujten ja 2ale, war nidjt aus bem 5̧aule getommen. Die Stiefmutter, bie

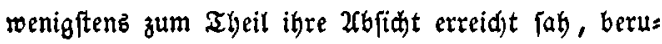
bigte fid am erften, und als Duvalb 2 ebrzeit vors über war, verließ audi) er baz şauz, unb ging nad) 9ariz zu feinen Xeltern zurüd.

Tुie aber erftaunte ber num zwei unb zmanzigs

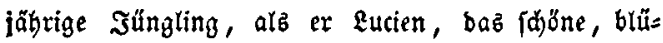
henbe Mäbrhen wieber jah. Sie ftürote mit ausge= breiteten $\mathfrak{2}$ rmen auf ihn zu, mit bem Rufe: 》mein

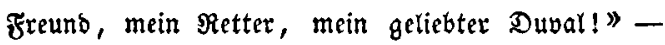
unb Dubal trat zitternb, errőthend zurüđ. Faft Dünte es $i \mathfrak{m}$ ein Frevel, ben $\mathfrak{s u}$ bes reizenden 2räbdens anzunehmen. Sein 2 uge hing mit unbes idireiblidhem Entzüceen an ibrer holben Beftalt, es folgte jeber ihrer Bervegungen; er fühlte, ein reizen=

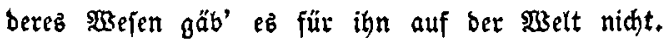
Uber ex hatte nitht ben Muth, ihr baz zu fagen; ex rabte nid)t, fie bei ber şanb zu faffen; bod ftreifte 
er von obngefähr an ibr Alcto, fo fubr ein füper Shauex burd fein ganzes șejen.

Seine Xeltern liebten \&ucien bereits wie ihr eiges nes Rind, obgleid ihre Rebbaftigkeit, ifr șang zu raujthenben Berguügen, thre Sudjt zu gefallen, fie zum diftern mit Sorge erfüluten. (Enolich, nadobm tie jungen $\mathbb{L}_{\text {rute ein }}$ Jahr beijammen gewejen waren, (id) täglid gejeben, (id hunbertmal ihrer Riebe vese fithert hatten, brangen bie Xeltern in Subal, bem Spiel, wie fie es nannten, ein Enbe zu maden, unb (iit) mit Sucien zu verbeirathen. 20ber sucie fagte

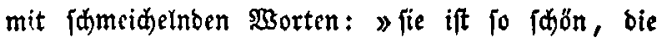
erfte 3eit ber jungen siebe, wir nod fo jung warum unb übereilen? " unb Duval wagte nidjt, etras bagegen einzurenben.

Da fam ber $\mathfrak{B}$ inter beran, und bunbert \&uftbara

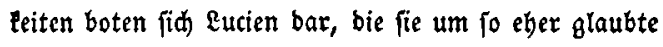
genieken zu bürfen, ba fie jest in Bejellihaft ihres

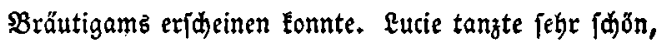
uno fo war balb fein Ball, wozł man bab jöñ Măbden, bie gute Tănzerin, nid)t gelaben bătte. So ungern Duval in Befellidaft ging; er fonnte bea 
\}üfen sitten ber Beliebten nidjte abjullagen, unb -nun jah ex, ber nie tanzte, nidyt btos wie sucie gleinh einem 3ephyr babin fowwebte, wie fid arte um bав holbe Mäbdyen brängten, fonbern aud) soie fie, glühens bor Freube, ign oft laum bemeréte, für jeben ihrer $\mathfrak{I} u ̈ n z e r$ aber ein bezaubernoes Räd)eln batte. Er ballte bie 5aände, er zog bie Stirn in büftere Falten; wilto rollte fein Zuge, inmer röther

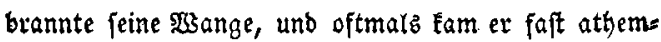
los allein nad) Şatife geftürzt, und [d)wur hod) unb theuer, fie nie wister fehen zu wollen, fie, bie ex fo grenzenlos liebe, unb bie both fein leben zur Sölle made! Rucie berubigte ing jebebmal wieber. J6ber bie Mutter fing an ben אopf zu idütteln, baß \&ucie nod) immer nidtb von einer, Jäbern Berbindung wiffen woulte.

Da brang Souval einmal mit ungeftüm in fie. > Eucie, 》 (prad) ex, 》entroeder Du wirft mein $23 e i b$, ober id) mus fort von bier; id liebe Did) biz zux Rajerei, entץdjeibe!" Lucie warf jidj mit Igränen

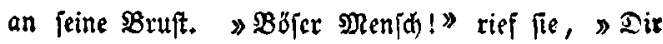
grügt nldht an meiner beipen Riebe; Dut rillt midt) 
quălen! SBab thue id benn, bas Did beuntubigen

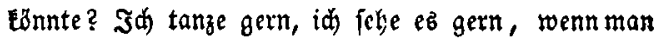
mid) aubzecidhnt; liegt barin etwas Bobjes? (Beniépe aud) $\mathfrak{D u}$ bie Zreuben ber Jugend, uno veridjeude die bufferen Falten von Der Stirn. Sth werbe Did niemals mit Eiferjud)t plagen, unb erwählft Du auth

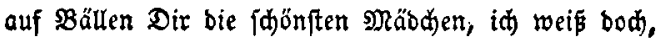
baß $\mathfrak{D}$ u mid) treu liebit. (Suter Duvar! laßs mix bod) die paar Jahre ber Jugend genieß̧en; ber Renz eines Mäbdens vergeht ohnebem fo fdnell ! Bin in fo alt, wie $\mathfrak{D a}$ jegt, bonn bat fid vielleidht Man des gelegt, und Du magft albbann bie ermfter wow bene Sungfrau zum Iraualtare führen, und Did, an

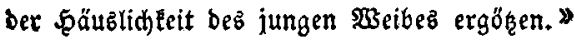

Diesmal gelang ez jerod sucien nidt, Duval zu berubigen; ex brang auf Entideibung. \(Entweber Irauung fajics für bie Rrije.

$\mathfrak{Z}_{\mathfrak{m}}$ andern Rurgen war Duval fort, unb bie Xeltem barüber untrobftidi. Sie ahneten bie utjade, unb überhäuften \&ucien mit Borroürfen; bie Nutter warb beftig, unb \&ucie, bie gebört hatte, bap bis 
298

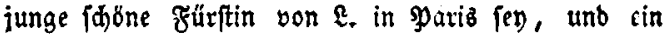
gebilbetes säbden judic, bas bei ifr tie Stelle eines Rammerbienerin einnebmen follte, meldete (ifi) fofort, gefiel und warb angenommen.

Euciene natürlid)er Berftand, und ein Goher Grab von Riebenswärbigfeit, ben fie fith immer mehr unb mebr anzueignen wupte, bie unermübete Uufmerkfam= Eeit, womit fie jebem Ieifen rointe ber Bebirterin zuborkam, vor $\mathfrak{a}$ llem aber ber reine Dialect, ben jie (prad), beftimmte bie Fiflitin fehr bald, Rucien ben Zntrag zu thun, fie folle bie Stelle einer franzôft: fden Bouvernante bei 5ortenfien übernehmen. \&ucie war auper fich bor Freuben über baz ganz unermar=

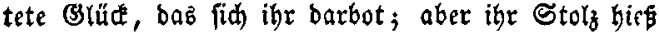
fie ihre Freube mäpigen. Gie Eüste ber Fürftin \$ăno, unb geftand ibr, wie fie nur aus untiberwinblider

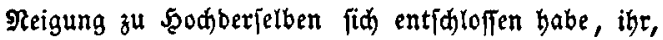
bie fie mit 2 nbetung liebe, fid zu mibmen. Die F̋̈uftin beftete bie fdönen 2 ugen gerührt auf Rucien, uno Mamiell \&ucie Bernon fam als Scortenfiens (Sous vernante mit ber Fürftin bier an. 


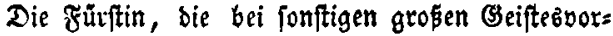
zŭgen gleid)wohl eine übertriebene Bortiebe für bab

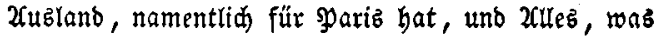
fie bebarf, won borther beziegt, ließ num vor einem batben Sabre bort neue Meublen uno Tapeten ein= Eaufen, um ben jogenannten Frembenflügel neu eine

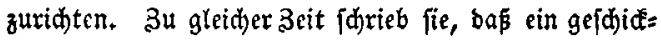
ter Iapezierer bie verlangten (Seräthfhaften begleiten folle, bamit unter beffen 2 ufficist $\mathfrak{Z}$ Ulez gut anlange und verarbeitet werbe. Die beftellten Sachen Eamen an, mit ifnen Duyar. Sey es nun, baß sucie von

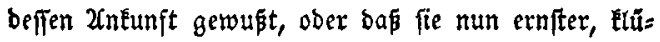
ger geworden, - genug Dubal betrat kaum baz ihm angemiejene 3immer, als \&ucie in feine 2 rme ftürzte. Er jegnete nun feinen Entjdylus, bieger gereißit zu

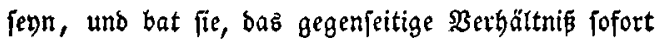
ber Fürftin zu entbeden, bamit fie beibe nad) vollen=

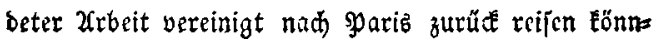
ten. Eucie 价üąte igr Berhältnif bei şortenfien vor, bat Subal zu fifweigen, und forwur itym hod und heilig, bas fie inn liebe, uno fobald er bie Krbeit vollendet, ifm forgen twolle. Dubal farwieg, obgleid, 
290

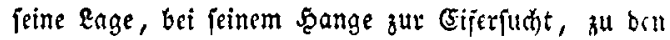

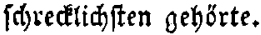

Eucie fpeißste mit ber \$prinzeffin Sortenfia, Rucie

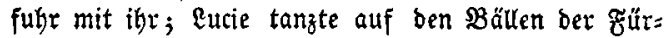
ftin, uno Dubal? - Jepobftenz war ez ibm exlaubt, vom weiten zuzufeten, wie Rucie, bas Göd)ft veizente Näbden, von ben anwelenten \$̧erren umflattert warb.

Jnber nahm fid sucie zulammen, und toie id)arf aud Dubal'z 2fuge fah, wie fhlüpfrig ber Boben

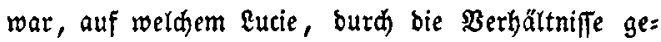
zloungen, ftand; Duval batte feinent. Sirunb, fie trgenb cines Feblers zu befhutbigen. (Sab es bod audh ber Xugenblicte fo viel, in weld)en lucie unbes merlt in Dubals Xrme idlüpfte!

Da Eam bor einigen Monaten Braf E... bier an, beffen Nadftellungen Rucie idyon in Pariz nur mit Mühe untgangen war. Er fah fie kaum, als er einen butchoringenden $\mathfrak{B}$ lid auf fie ridhtete; Rucie fhlug erröthend bas Zuge nieber. Der Graf, ber in \$aris gewöhnt wax, fie als ein $\mathfrak{B}$ ürger = Măbdhen z̆ begandeln, ftiminte ieg̨t bie Saiten böber. (E) 
rame fie Gräutein von 2 emon und fübte ibr mit

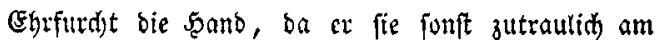
Rimn gefapt. Rutie füblte fith gefdmeididelt, zitterte aber, fo /oft fie in bez frjöncn Mannez Räbe Eam. Dod) in einiger 3eit warb fie zutratulid, ba fie fah, wie feine $\mathfrak{x h}$ htung fith gegen fie mekr und mebr er: bobte; fie glaubte am Enbe, er habe fie nidit wie: ber gekannt; fo fremo, fo fein uno zart begandelte fie ber Bitaf.

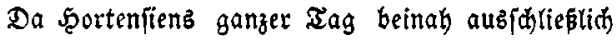
ben Rehriftunden geivibmet wurbe, fo war es Sitte,

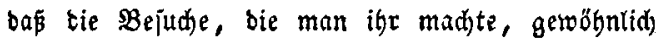

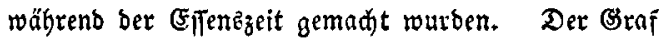
fand fith täglich um biefe 3eit ein, und wärzte burch

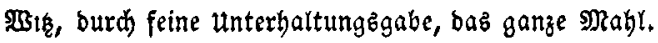
(Einmal traf ez fich, Das gerabe über eine Szeirath gefproden warb, welde ber Baron St. mit einem

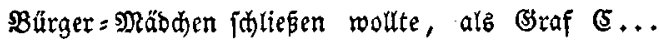
herein trat. "Şelfen Sie miv, 》 rief ihm bas alte Zrăulein sernwille, der sprinzelfị Soofmeifterin, entgegen - 》helfen Sie mir, lieber Graf! der (Seheimerath nimmt bes Baronz şarthie, und it) 
292

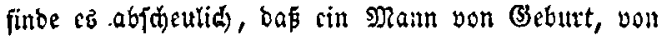
silbung, von \$erftand, jidh fo febr vergefien Eann, burdh eine 5eirath ber 2frt feine ganze Familie zu be= făimpfen. 》

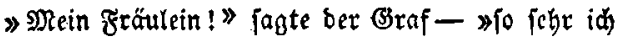

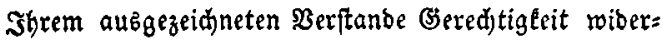
fahren laffe, id mup in biejem spunet der Meinung bes Beteimiaths beipflithten! - Blaubt ber Baron,

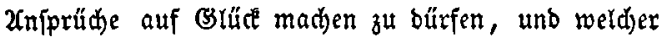
Nenidh glaubt bieb nid)t? fo mus er auch ftanbyaft ben $\mathfrak{B e g}_{\mathfrak{g}}$ berforgan, ber b̆丶 bem führt, waz igm alz

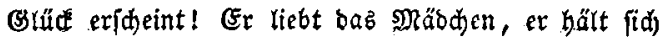

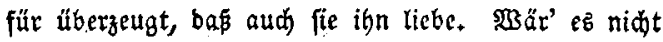
Raferci von ifm, wemn er bem einzigen wahren (3) iü, bas wieberum einzig in wahrer liebe befteht, entfa= gen wollte, weil jeiner Beliebten ein Stammbaum, cin berügmter Same feblt? 》

》 Giebt ea wohl, 》 fuhr er mit Begeiftrung fort,

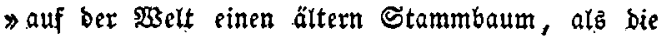
Sugent, bie Sdyönheit, bie Riebe? cinen ältern Namen, als Seliebte, Sattin, Mutter? $-\mathfrak{D}$ mein Fräulein! id fenme ben Baron nidbt, aber id adte, 
id) liebe ign um ber Feftigkeit willen, mit welder er alle Đämme bcr Sonvenicnz burchbrach, um glüctlich

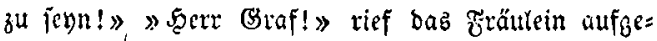

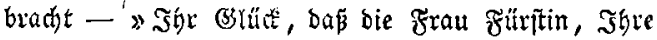
Durdjlaudtige Coufine, biefe Brundjäßze in Shnen nid)t aburt! \$\$3aum?" frug ber (Sraf - 》glauben

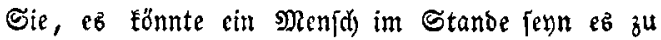
Gimbern, baj ich co nodh heut' bem Baron nad)= thăt? - D เoär' id) geliebt, wie ber Baron, 》Fef̧te er mit bewegter Gitimme binz̆l, und fein gropeses

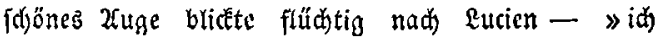
würte, wie $\mathfrak{E r}$, alle $\mathfrak{S}_{\mathfrak{d}}$ ranken bes 5̧ertommens

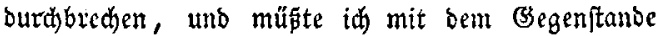
meiner Riebe in einen anbern $\mathfrak{3}$ elttheil flichen! $\Im^{d}$ wärbe $2 \mathfrak{d}$ m entjagen, nur meinet sivbe nid)t!»

Eine Minuten lange spaufe folgte biefem vegei=

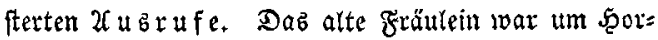
tenfiens witlen in Berlegengeit, bem (Gefprüd)e Dieje ŞAenbung Begeben zu haben. Dex (Sraf fdien in Nadbenten verjanten, unb Iucie? $^{-}$Mit podjenber Bruft, mit glühenden $23 a n g e n$, mit niebergeidhlages nen 2lugen jaß fie ba. Sie batte bie Gremmenbent 
25.4

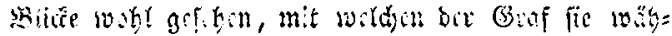
rinb jeiner SRebe faft burd)fady. Seine Sisorte, jeine Xufmerkjamkit auf fie - ncin, es war kein äroci= frl, ber (S)raf, ber fäöne, ratide, gefcierte (Braf, mus̄te fie licben.

Nit Mäbe nux crzwang fie faffung bei aifac,

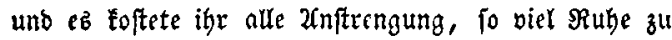
erringen, bus ber Braf ihte Befüthle nidht erratgen möbte. Zutein Exum war fie auf ihrem Bimmer, atz fie ben wiberitreitenoften (Empfindungen fid) bingab.

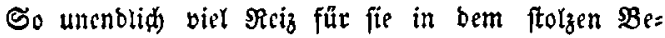
wuptieyn lag, fict) von bem Girafen geliebt zu wij= jen, burfte feine Riebe fie freuen? fonnte fie jemale rin Beftänoni” von ibm anhören?

Da fam ber Braf, fie zu einer Ueberrajdung zu überreben, bie ber Fürftin gelten follte. Lucie war

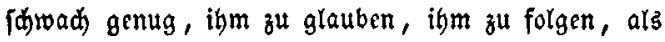
et fie in einen entlegenen rhcil bez spark'z fügrte. sisber war fie nie allein mit ihm gewefen. ફ̧ier enstid, umjojattet von alten (sid)en uno Budjen, in balber Dämmerung, weit genug vom Shloffe, dap Niemanb fic bören, Riemano fie fegen fonnte, warf 
(4) Sat Graf vor ifr nieber unb fduwn, baf er fie licbe.

Eucie wollte entiliehen; umjonft, ser Graf um: fopte igre Anice, er bat, er befdivur fie, und aucie-

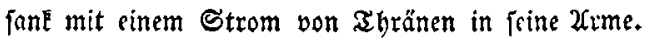

Dor Braf brang nun in fie, fie öfterer allein fiben zu bürfen, unb aucie ragte ez nidjt, ifm ifor Berbättniß mit Duval zu geftehen, aus Shaam, daß

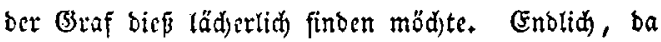
ber (Graf nitit aufbörte, fie zu befitürmen, verjprad) fie, ibm in einigen Iagen cin 3eidicn zu geben wemn uno wo ox fie fpechen tönne.

Dab 3eifhen warb gegeben und verftansell. Sie faben und ipradien fid biter, tno immet roill \&ucie Dubale Rumen nennen; both immer verjiegelt bie Shuam thre sippon. Da ftcht cinmal Rucie mit Sertenfion auf bcm Savillon, ala ber Graf aubreitct.

$\mathfrak{S a} \mathfrak{a r}$ cen in jeinem Enappen, veidgefticten Jagoltrios ben muthig ftolzen Englänoer belteigt,

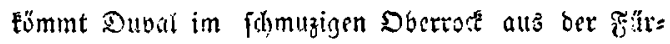
flin Binmin, wo cr gearbaitst gat, tum in foine

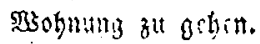


296

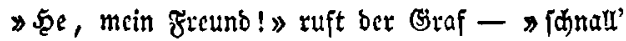
er mir eirmal ben $\$$ üget Eürzer!" Dubal gehordt, indeß ber Graf ben Damen vom Yyfore Ginauf Rüfife zu wirft. - Eucie ftand wie vernightet. Eie füblte fidi) Durdh Duvals untervürfigteit gebemüthigt; fie hapte ihn um jeiner Bereitmirligkeit willen, uns als er nun ben. Befeht bes Grafen exfüllt hatte, warf ibm biejer ein Berbftüa zu. - Sun fonnte fid Qucie nidit mehr balten, fie fdymante ins Simmer, fie zitterte, fie wagte nidit, bie ftolze fdophe Beftalt

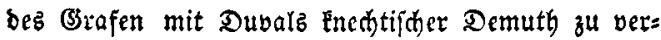
gleiden; fie Eonnte ben Geoanfen nidjt faften, bas

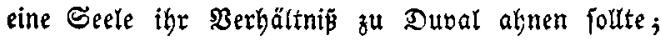
fite jöämte fid feiner, fie faß in finftern sräumereien verfunten. Froft uno seige wed)jelten in igrem $\mathfrak{s}=$ netn; fie fubr zufammen, alb fie enditid Jubends bes (S)refen Stimme in ben 2 partementz der Sprinzeffin bürte; fie wollte auf ihr 3immer eilen und fonnte nidft fort. Da trat ber (Graf Ginter ihren Stuht, uno id)meidyelte Yeije: skucie, um zehn $\mathfrak{u b r}$ auf Ingrem 3immer, nue einen 2(ugenblide! 》 


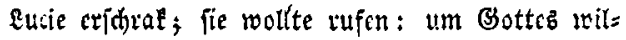
len nidt! aber bor Graf war veridyunden. Jle Eeaz podte vor 2(ngft. - Enblid) follug eb balb zegn uhkr. " Gott," benft fie, ”wenn Duval? 》 Dei Bebanke übaridüttete fíe mit Eis; fie fühlte,

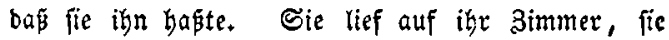

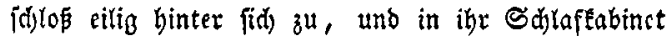
eilens, wirb fie bon bem Brafen umarmt, ber ver

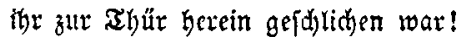

Sucie jane in namentofem Sitmerz auf's Sopha; ber (S)af bot alle אứnite ber Beredfameit auf, und Duvals geträumtes Bsiüč̉ ging auf ewig unter.

Bon biefem 2lugentiblite an waro Rutie fälter gegen Dubat; fie rouste nun, bap fie ben Grafen mit

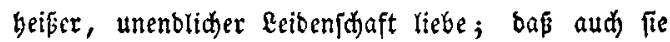
eben fo getiebt reerbe, hatte ja ber (3raf Eniend ge= idiworen. Sie redinete auf Duvals Edelmuth; fie wollte ihm ift Bebeimnis anvertrauten, fie wolut' igm ihre siebe zum Brafen entocken, unb ihm jagen,

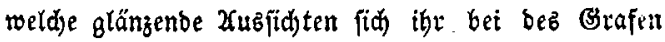
Grundjäzen öfineten. So feft fie überzeugt war, es würbe ifr gelingen, bert glühenden siebgaber 
208

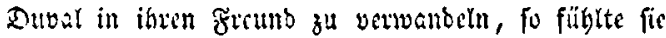

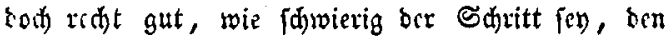
fite of thun im Begriff ftand, und mit weld)er 3art= hrit bas bibher beftebende $\mathfrak{B a n d}$ gelöpt werben müfe. $\mathfrak{j h r} 3$ uftand wato um fo peinliçex, je weniger fis rilte, fich mit Subal zu verftänsigen.

Der (3raf bebandelte fie feit jenem Xbenbe mit

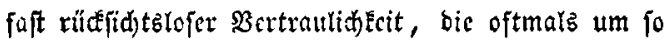
ntchr verlesend für fie warb, ba bee alten Fräuleims

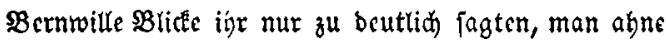

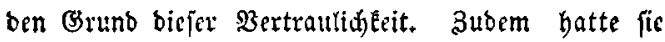
faft nidft mehr bon Muth, ihr 3immer zu vertaffen, weil Subal igr übexall auflauerte, um fie zu fpres Фen. Jmmer fóüttelte fie verneinend ben Sopf, retm ox aus ber Ferne igr bas brfannte 3ridien gab, fie diejen 2lbend jehen zu wollen. Enolid tam fie cines 2bends aus ben 2upartements ber Fürftin, bie 2ugen voll నhränen, bie saruft zerrifien von ben quälen= ben Shmerz ber fürditerlidiften (Eiferfudit.

Eine junge Dame aub bcr Nabbarfdaft war bic= frn 2ubend in ber affemblee grwejen, uno Rucic muste

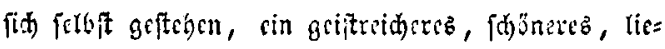




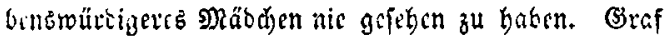
(5... Gatte nur 2 uggen für fie gebabt; feine ganze

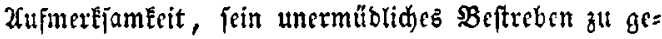
follen, war hut auf fie geridet gewefen. Bergrters Fatte \&ucie fid igm einigermal gerähert, vergebinz mit biefer ober jener Frage fein (sefpräd) zu unte:= bred)en gejucht. Der Graf hatte fie immer mit kals tem, ftolzem shid angefehen, und jein Bripräd mit ber jungen Dame fortgcjegt.

Eucie tif die $\mathfrak{B l u m e n}$ aus igrem $\mathfrak{s a a r , ~ f i c ~ w a r f ~}$

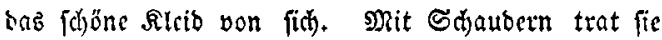
vom Spirgel zurüde, als fie ilyre von Sdjmerz unb शzuth entftellten Büge barin crblickte; fie brad) in Intes Sofludzen aub. Mit rojd)en Sdbritten, alb wollte fie ihrem Befühl entlaufan, ging fie im 3im= mer auf und ab. Da fdrid re licije itser ben cor= ribor. Nazar's möglid? badate fic - ber Braf? und mit unaubipredylid)er Schn[utht blictete fie nad bet Ihür. $\Im$ hr $\mathfrak{B u f e n}$ arteitete heftig, alle sjulfe flopften; ba b̈fnete fich bie aghür; fie ftürzte bem Eintretenden entgegen, und fank - in Dubrts Xrme. 
Shre Lage war fürdtertid. Sie burg bas glü= benbe (sefidit inz Sopha; fie rollte fid) jammeln, um rubig mit Duval zu fpreden; fie vermoctete nicht. (Ein tiefer Geufzer arbeitete fid) mübjam von

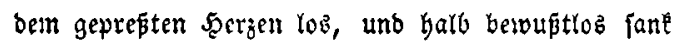
fie von Reuem in Dubale Urme. 》Neine sucie!" fagte endlich bicfer! 》meine füpe Rucie, $\mathfrak{D}$ u bift überrajd)t, bas id jo fpät żu Dir fomme, $D$ jütme mix nidut; idf fonme in ber böthffen freube; meine

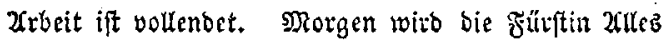
befenen, mich fürftict) belohnen, unb bald - o bald fegren wir $2 \mathfrak{r m}$ in $2 \mathfrak{r m}$ zu meinen Eitern zurüd. Sich, meine Rucie, biefe Sic(j)rid)t Eonnt' id Dir nid)t vorentfalten; batum befudt' id) mein Bräut= ben nod) fo fät am 2(bend!" Rucie entwanb fid fei= nen Xrmen; jie rang verzuveiflungsvoll bie ફ̧ände; fie heftete bie ftarren Xugen an bie Decke. Da fragte Duval: 》 $\mathfrak{B a s}$ ift $\mathfrak{D i r}$, Eucie? $\mathfrak{D} \mathfrak{u}$ liebft mid) boch nod)? \#- und als elucie immer nod be= regungaloz da fá, fapte Duval mit roltendem Xuge, fie will beim Urme. \Eucie, tief er - v rebe, 
toas ift Dir? liebft Sou mid), unb foll id morgen mit ber Fürifin jpred)en?»

İçt fam Iucie zu fid felbft, und erjaraf vor Duvals wilper Bebchrbe, vor feinem lauten Xubrufe, ba fie nur einige ̧immer entfernt bon Sortenfien wohnte. Sie bat inn zu jaweigen; fie wollte zu reben anfangen, aber Soubal frug mit gräplidjer Sälte: „ Lucie, liebft $\mathfrak{D u}$ mich, uno foll id mit ber Fürftin fprechen, ober nidgt?»- \&ucie, um ben fdreditiden Menidien fdnell von fid zu entfernen,

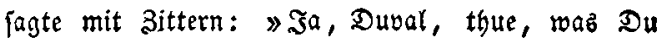
willf - aber verlaßs mid beut'; id bitt fehr ange= griffen, fehr erane." Dubal warf cinen langen, fürch= terlidien Blite auf fie, und ging.

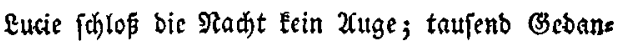
fen zermarterten ibr (Sebirn, taujenb fomerzlide Be: fühle ibre Bruft. Riebt mid) ber (Graf wirklid? badite fie, - wie wirb er eb jemals vergeffen föne nen, saß id mit Dubal, mit einem gemeinen Mens

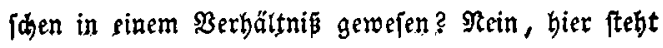
alles auf bem Spicle; entrober 2 ulez getoonnen, ober Xufes verloren! Der (Sraf barf nie abnen, baß 
302

id) einen Xnternl, als ibn, jemale geliebt babe! Sie zog (id) fehr früh an, fie ftürzte in ber Fürftin Sof)laf: gemad, und warf fith vor bem Bett auf bie Rnies.

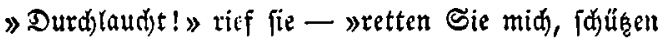
Sie mid)! Entfernen Sie mid j(jnell von bick, bevor

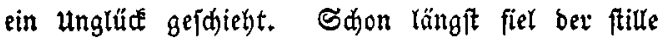
Irübfinn bes \$parifer Sapezierer's Mebrern auf, unb aud (Ew. Durd)laud)t gerubten vor einiger asit bie Bemerfung zu marticn, wie bex junge Mann einem ftillen $\mathfrak{3 a h n f i n n i g e n ~ g l e i d ) e ~ - ~ e r ~ i f t ~ w a b n f i n n i g , ~ ( E w . ~}$ Durdlaudt! ex bilbet fith ein, mid) zu lieben, von mir geliebt zu feyn! Jegtt will ex mid gar Geirathen!

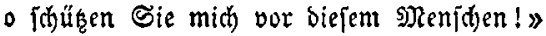

* Sey rubig, meine gute Bernon, 》) Fprach bie gütige Fürftin - 》Du ftehit unter meinem Sduse; Dir foll Eein Reid zugefügt werben. 2Cber Du bleibft bei mir; nid)t fort follf $\mathcal{D u}$; Gre foll es; benn feine Urbeit ift ohnebiek bollendet. (Seh' auf Dein Bim: mer, unb verlaß es nicht, bis ber unglüdelidje ent= fernt ift. ten iff.

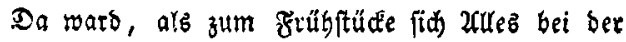




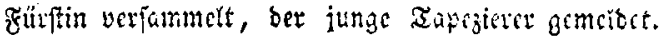

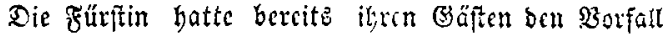
mitgetheilt; mon bebauerte oen fleipigen, talentoal= len jungen \$rann allgemein; nur (Biaf (5... zinei= felte an ber şafarigeit ber Sadje; er bat jeine (5ou= fine, ben jungen \$rann vor fich zut laffen, uno fei= nen 2fntrag wenigftens ruhig anzuhören.

Mit cinem kleinen Sdjauber jeşte fid) bie Fürftin binter bie anweienten Serten und Damen, und Du= val trat ein. Mit rufiger $\mathfrak{B}$ ürbe lagte er erft, wic feine 2rrbeit nun volfehdet, unb zrar, wie er gewis glaube, zu 2llter 3ufriebengeit; bann bat er, ivo

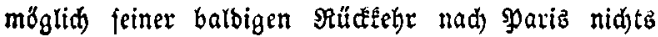
mekr in ben mit göd)t rügrenden $230 r t e n$ jeine Riebe zu Rucien.

Die Züurftin lädjelte nid)t obne Berlegenhait; ent= (id) jagte fie itm, baj ex, potalb er wolle, abreifen E⿱艹 baßs aber Rucie fid) unter ihren Sdứ begeben uno ine idhon zatez entbect habe. (Ex möge nod) beute Ron Repos verlafien!

Der junge Mann verftand fein $\mathfrak{B}$ ort. Endida 
302

id) einen Jnecul, nts ign, jemals geliebt babe! Sie zog fid) fefrr früh an, fie ftürzte in ber Filurtin Soblaf= gemad), unb warf fith vor bem Bett auf bie Aniee. 》Durd)laud)t! 》 riff fie - »retten Sie midh, 「düügen Sie mid)! Entfernen Sie mid (d)nell von biex, bevor ein unglü geídiegt. Sdjon längit fiel ber ftitle Irübjinn bez \$arifer Tapezierer' \$Pebrem auf, und autf) $\mathfrak{E}_{\mathfrak{w}}$. Durdjlaudt geruhten bor einiger 3eit bie BemerEung zu madien, wie ber junge Mann einem ftillen ßahnfinnigen gleidhe - er ift wahufinnig, (Ew. Durdlaudht! ex bitbet fid) ein, mid) zu lieben, yon mir gełiebt zu fern! Jest will ex midy gar heiratyen!

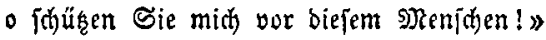

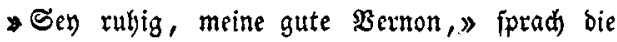
gütige Fürrftin - 》 Du ftehft untex meinem Sduge; Dir foll kein Reib zugefügt werben. 2aber Du bleibft bei mir; nidt fort jollft Du; שre joll es; benn jeine Urbeit ift ofnebiez yollendet. Geh' auf Dein 3im= mer, und verlap é nicht, bie ber unglüdtid)e ent= fernt ift. 》 - Eucie ging, aber alfe frurien folg= ten igr.

Da watb, âb zum Frütftüdé fid Xlleb bei ber 
Fituffin verjammelt, ber junge Tapioierer gemcitect. Die Fürfin hatte bercitz ireren \&äften ben Borfall mitgetieilt; man bebauerte ben fleipigen, talentost= len jungen spann allgemein; nur Braf (5... zaweis felte an ber şahrheit ber Sadhe; er bat feine (5ou= fine, ben jungen salm vor jid zut laffen, uno jei= nen 2Intrag wenigftens rubig anz̧uhören.

Mit cinem Eleinen Sdjauber feşte fidh die Fürttin binter bie anterenden 5̧eraen und Damen, und Du= yal trat cin. Mit rufiger $\mathfrak{B u ̈ r b e}$ jagte er erft, wic feine $\mathfrak{Z} r$ beit nun vollehbet, unb zwar, wie er gewij glaube, zu WuKer Bufriebentheit; bann bat er, tov

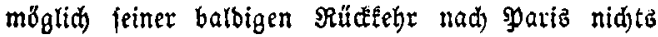
mehr in ben 20 eg fu legen, unb entoedite enblid) mit hödft rührenben \$orten feine Riebe zu \&ucien.

Die Fürftin lädelte nidt obne Berlegengeit; eno= lid jagte fie inm, baj er, fohald er wolle, abreifen $\mathfrak{f u ̈ n n e , ~ b a s ~ e r ~ i h r e ~ v o l l e ~ 3 u f r i e b e n h e i t ~ m i t ~ f i c h ~ n e h m e , ~}$ baß aber Rucie fid) unter ifren Sdus begeben uno

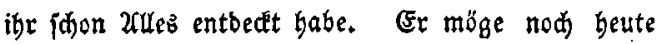
Mon Repos verlaften!

Det junge Mann verftand kein SBort. Enblid) 
aufgemuntert burd) bez (Siafen 2Cuzruf: Mamfell Rucie jollte bicr gegentoärtig fern! erjudte er barum, bap fie gerufen werbe.

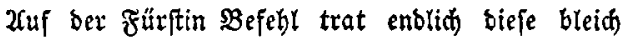
unb sitternd berein; ibr Blid mies fo viel möglid ben unglüdliden. Da rief er mit rübrenber Stimme: $\rightarrow$ Sucie, id bitte Did, , jage ferbft, wie lange wir uns fajon lieben, wie oft id) Dir, Dou mir Treue gefdworen, wie nod) in ber verwidhenen sadut Dus mix verjprodicn bajt, mit mir zu reijen. 》 bok furdtiam ibr Xuge, und geroabrenb, wie auf

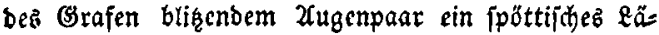
betn f(b)ebte, ftürzte fie zu ber Fürftin Füpen unb rief laut und verzmeiferno: „Er ift wahnfinnig! o

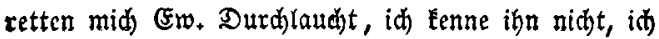
lieb' ign nidbt; id hab' ign nie geliebt!s

Fin gräplicher Schmerz orang burd) Duvals Seele; ex ftand exftarrt; er Eonnte nidjt reben, er beftete Die flieren 2 ugen auf eucien; jeine sBruft arbcitete beftig, fanell bebte ihm jebc suber, feine Sănoe ballten (fid) feft zujammen. Mit నobesangit gab igm die Fürtin Befebl, fid) 孔u entfernen. (Er mupte 
weggefüht werben; benn er war befinungellos. Der Sammerbiener bradte ign auf fein 3immer; cr blieb ftumm, er ap und trane nidit, und faien ben garzen Iag/ völlig bewegungbłoz.

(Enblid am zrotiten Sage ging er an feinen Sareibtifd), öfnete die Brieftaja)e, fans Ruciens silb, und hialt es med)anijh bem Rammerdiener hia. Diejer fagt: " $\mathfrak{a}$, guter Dubal, id fenne es

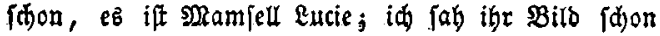
beim Brafen. $)$

Saum bat ber Rammeroiener bie গ⿹丁口丿 gefpro= den, als Duvatz $\mathfrak{x}$ uth fürdterlid) auzbridt. (Er ftürzt burdh bie Rühe, wo er sine 2rt exgreift, nad Sortenfiens Bimmern, wo er Eucien, wo er ben Bras fen zu finden hofft, und würbe fidherlich, Dieje ober Jenen geopfert baben, rär er nidbt mit bem $\mathfrak{F}$

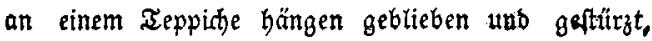
worauf man fid) fogleidh feiner bemähtigte, unb ign, beffen Raferci nun zum bölligen 2 ubbruch eam, auf feinem simmer gebunben einjdlof.

Der Graf bcurlaubte fih bei ber Förftin unb $g a b$ eine Alcine febr eilige Reife vor, zuvor aber jagte or 


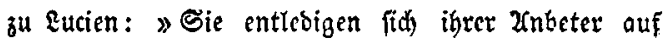
eine graujame 2rt. Sie verleugnen ben jungen fajo: nen Mann, mit bem Gie, wie man Gört, fơn halb und balb am 2fltare geftanden. \$rab würoen Sie mit mir madyen, wenn id mid) nidt flüglidh felbft zurüce zög? - Senn Sie in 3utunft anfãnglich roeniger gütig gegen Jigre Riebbater, fo werben foldje gewalt= jame Mittel binterber nid)t nöthig rerben!»

Eutcie bot inren ganzen Stolz auf, ihre ganze Siraft, aber bas Eaden miflantg, bas fie bem Bras fon natjidjicken roollte; traftloz fank jie in fid felbft zufammen. "Ieufell» ftögnte fie, alb ber Braf vom Sofe fubr. Sie zroang fih an ber Iafel ber Prinzeffin ruthig zu expheinen, obgleid eine ganze Sölle in ifrem $\mathfrak{B}$ ujen loberti. Der Shblaf floh igr Jutge, fie fpradt irre im Iraume und träumte im

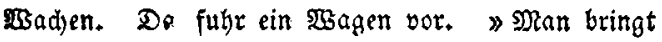
ina weg ! 》 fdrie bie Rammerfrau, unb Eucie wantte

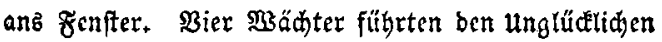
in Setten feft gefoloffen. Seine Stin, feine Sänbe olufeten von ben fdweren Eifen; man bob ibn in 


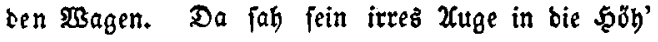
unb Eucie fubr voll Entjeken vom Fenfter zurük.

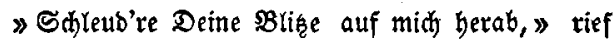

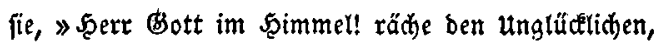
befīen blüthenvolles Dafenn ich vergiftete!》 Gie riß fid) bie $\mathfrak{B r u f t}$ auf und fties tief ben Dold in :igr berz, ohne baj ez bie babei ftehende Sammerfrau verbinbern Fonnte.

St. ReIty. 
Das neue Ieben.

$\mathfrak{R a d}$ (Songora.

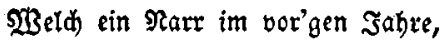
Bin id heu'r aud) nicht ber Riügfte! Biel vermag bie Meberlegung uno bie zeit vermag nidht minber.

Jener batte Redft, ber fagte: Brỡ fitig wie ein alter Sdiefer

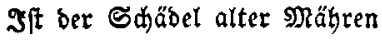
unb ber Sđäbel ber Bcrtiebten.

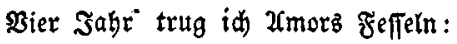
wahrlid)! adte bient' id licber Jutf ben $\mathfrak{z}$ ürtijden Baleeren, In ben Nertern ber 2Ulgierer.

Zag für $\mathfrak{Z a g}_{\mathfrak{g}}$ war meine (Eiferfud)t und leere (Brillen, 


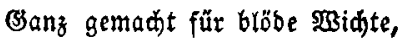
Die ztwei $\mathfrak{X}_{\text {ugen }}$ überwinden.

2uzl ben Garten Sclabenbanbea (Jährig wito es im 2uprile)

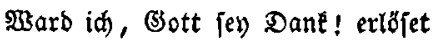

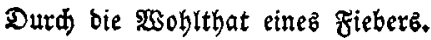

Diejem uebel bane' id einzig Meiner Freiheit füßß̨) (Süter und das id Eein Rarr mehr bin, sBin id juft aud nicht ber Rlügfte.

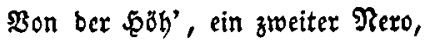
Sdau' id) zu, wie Znore fdrwiken In ben jđnöben Riebesflammen, 230 Die Egr' in Raud) verflieget.

und id bin fo barten Şerzens, Das, wo (onft id milden Sinnes Sdrreient bei geiprungen waäre, Sharend id binzu mid ftürze. 
Scit jehn Mronben jathlaf id wirber; Denn fo lang' ift'b, bas id liege,

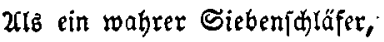
Bie ein Sad auf meinem 9 füble.

Mid) ftört feine Gritl' im Sdhlummer, Sein 'Setkeif' mein $\mathfrak{O h r}$ umidroirt, 2Cbenbthau mid nidht verkältet uno mein $\dot{B}$ eutel bleibt gefüllet.

Freunbe fab' id fo viel, bap id Stets alleine Eamn fpazieren, Wreil id nun Éein Narr mehr bin, Bin id juft aud nidit ber Rlügfte.

Meine Büdex müffen jeço Mix bie Maientage kürzen unb bie falaffen, wiberwätt'gen Sännernäđte mir verjüpen.

\$Büroigt mandhmal meine Mkuje Jott 2cpollo feiuer ફ̌itlfe, 


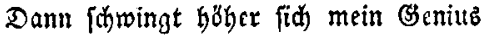
und id (dareibe mandjes nieber.

Dft laud manbl' id 'burd bie Fluren, Dá bie Seele nid)t ver joimmle uno ber (seift burch Frü̆łingęfräuter Sid) befreie won ben Brillen.

und.baheim in meinem Simmex Nebm' id mandimal eine 3itter, Die, wic ein Barbies, idf ftimme und, wie ein $\mathfrak{B a r b a r , ~ i d ~ j p i e t e . ~}$

So täuju' id hintroeg bie Stunbon, Die behaglidit mir verfliesen,

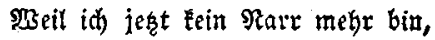
Bin id juft aud) nidht ber Rlügfte.

Sonft verforgte midy, ben agoren,

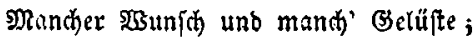
Dodh jegt bin ith in bem xempet Der Enttäujđung ganz gefidert. 
Đier an biejer Freitheitfätte Scat 2 fftolfo $^{*}$ ) mir vertiehen Ulle Tropfen jaincs Fläjddens, und bie Atugheit ihre Britle.

Dieje zeigt mír, wie geftüget Uuf ben Stab von einem ફ̧ixten uno bas 3epter eines Raijerb,

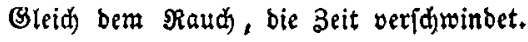

Seit id) in mein snntes idaute, \$3arb id biefe 2 abrheit inne, und ein Marr bin id nidyt mehr, Bin id) juft aud nidt ber Rlügfte.

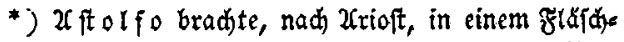
d)en aus bem Monde dem tolken Roland feinen Bers

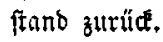

SBeauregarb gandin. 
ङalaraffenland.

Jeso, ba mir bie Buitorre

Dient als robllautbolle Sprade,

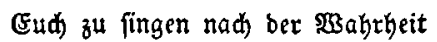
Bon bem wunberbaren Lande:

Denn bevor id, furz uno bünoig, Ireulid)en Beridht erfitatte

Bon bem \&ano, bas ibr betretet, Son bem Bole unb allen 2Unoern, şiffet, baß wir ঙnjulaner \$ur in ber (Suitarxenjprade Immer uns vernebmen laffen; So poetijd) uns phantaftifh, SBserthe Freunde, ift diep Eiland, und Sdlaraffenland fein Rame; 2ud) wirb's Nirgendzbeim gebcifen, Dean es führet bribe ramen.

Sier zu Lanbe fino bie Rlifte Rauter 3ephirz, zu erlaben 
Nit bem Boblgerun ber Rojen unire nimmerfatten Rafen.

Răbl, Eriftallbell find bie $\mathfrak{B}$ affer, Durd) bie 2u'n fliept Rimonabe und ez fteben foneegefüllte $\mathfrak{B}$ einerfificter allentbalben.

Uuf jenfeitigem-(S)ftade (Siebt eb $\mathfrak{B a ̈ u m e , ~ w o ~ f a t t ~ B l i t t e r ~}$ Ein zart Milubbrot bei bem anbern, uno, a!s Früd)te, Suchen bangen.

unb ber Rern von biefen Frübten Sinb Paftetden, bie als Bormabl

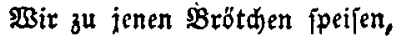
um zu reizen unjern Baumen.

Einen Baum giebt's, ber fo grop iff, Dap er unter jeinem Shatten Nicrzig taujenb Tafein Raum gicbt und an jeder zroanzig Sd)maufera. 
Seine Früd) finb fajanen,

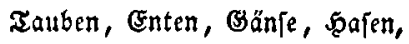
Eämmer, Şammel, Inoiane, *) Sัunge Sägner uno Rapaunen.

Zule wadjen bort gebraten Sber fo zured)t gemadhet, Daß ber Baum jorint ausgeftattet \$ie mit æöpfen, fo mit Syannen.

uno wer $\mathfrak{p l a s}$ nimmt an ber Zafel,

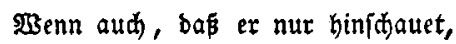
\$3ornad) feine âung' gelüftet, Siehft bu's glcid ins Naul ibm fabren.

3u (Sebote ftehn fed) Măb(t)en Jeglidjem von unz Shlaraffar, Fine grop und ablernafib, Stumpfoenartt unb Elein die Znore,

*) Inbia n, פuter, calccutijher Şahn. FBie= nerift). 
Sdywarz geaugt und blonb bie Dritte, unb bie Bierte blauen 2lugez, Eine Sdilanke, jajalkhaft läblyelno, Eine Diffe, immer ladjend.

und man nimmt un jebe \$sode Diefe Sed's und giebt' une 2rnbre. 3ieht nun WUle, Şanb in Sand, Jaudzeno inz Sd)laraffenland!

Beauregarb gandin.

\section{Da $\mathfrak{P} \mathfrak{i} \mathfrak{i} \mathfrak{i}$ legium.}

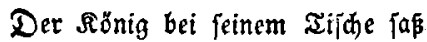

uno eifrig in alten sapieren $\mathfrak{l a z}_{\text {. }}$

E6 waren Documente; fo eben

Jhm von bem Ranzelar übergeben,

Die 24Uez entbielten, waz in bem $\mathfrak{E} a$ ad

Bon alten syrivilegien beftand.

Der אünig fdüttelte öftermal 
Das Saupt, burafichenb die grope 3abl,

(Frvaagend bie unbebeutenben (Brünbe,

Dzodurd) einft to Mand)er mand) Redt fith verbiente.

$\mathcal{E}_{5}$ meinte, mas man fo Einem exlaubte,

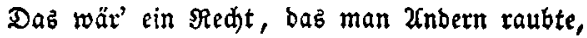

uno war bez $\mathfrak{w}_{3}$ illene, bie meiften (Sebräude

uno Borredite abzujdaffen im Reidje.

unb unter bem vielen peltjamen sanb

Sam audh ein Dofument ihm zur Şanb,

Das Srleanb $\Re$ Rathaberrn für emige 3eiten

Dafür, baj bie Stabt cinft vom Feins' fie befreiten,

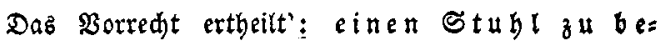
gebten,

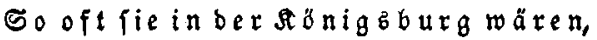

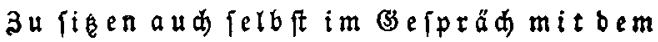
凡önig.

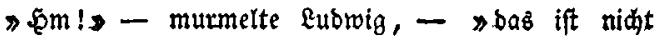
wenig!

Die Serren von Srleanz, mus i千 geftehn, Sie ban gen (Frmulung fid) vorgerehn! Fुir wollen boh jehn, wenn fie wieber exideinen, 
Ob fie wirktid fo idwath find auf inten $\$$ Beinen, Dak ferbft vor bez אiönigz Majeftät

Nidht Ciner mebr feft auf ben Füpen fteht.

Sie follen, - id fdüwö's, - mix baz Siken vergeffen,

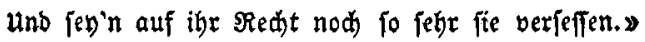

2(d)t Tage waren vergangen faum,

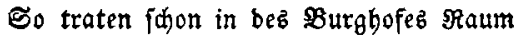

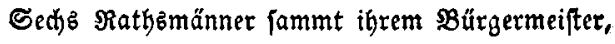
Die erftern bübid feift, ber lek̨te now feifter, uno jagten, bajs fie von Drleans fämen,

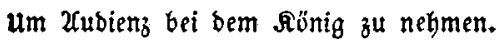
CE melbet bieß einer vom 5̧ofgefinbe Dem Rönige; biefer befiehlt, baß gef(t)winde Die Stügle, Styämet uno alles Ģeräth,

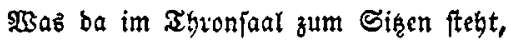
In möglidhfter Eile hinausgebradt werbe,

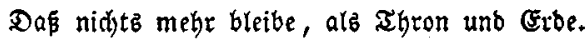
Uta bieß war gefdehn, nahm ber אönig fein Den $\mathfrak{g l a c}$ auf bent Igrone fdjnell felber sin uns man liep bie Şerrn in ben Gaal berein. 
Sie famen und neigten fid) Sreimal tief, uno, bentend an ifren Frcibeitabrief, Sabn fie in bem Saale fid) ringz herum Nač) zubereiteten Stühlen um; uno immer ängftlider wurbe ihr $\mathfrak{B l i d}$, . Sie fd)auten vorwärts und idauten zurück, Sie råufperten, hufteten, wifłten ben Sd)wei Sid) bon ber Stime, die brenneno Geí, unb büaten fid intmex uno wăhreno bem Bücten Berzauften fie zornig bie Snotenperücken, und fauten immer und jowiegen immer unb fanber nidjt Rettung im banzen 3immer.

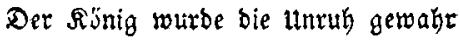
und lächelte beimlid und (prad) bann zitt Cdjaar:

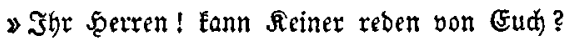
Shaz wollt ihr son mir? warum feht ihr fo bleidy? und Reiner wagt' eb, bic Stimm' zu erherben, um Driennz Sorred)te nidhtz zu vergeben, und neuerbing judten voll 2lngft und פual Sie ringaberum in bent ganzen Saal, Db aud nid)t ein Eleiner Sdämel fid finte 
320

Der ifrem Recht zu Sebothe ftünde.

D Gätten fie nutr einen eing'gen erblitłt, Sie bätten fid all auf ben einen gebrïldt!

Dab bauerte viele Minuten lang,

Den Şerren rourb' immer megr angft unb bang,

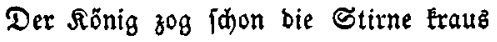

unb rief: " 3ur Sad'! bie (Bebultb geht mir auz !

$\mathfrak{D a}$ fapte in feinem tiefen $\mathfrak{B e r d r u f}$

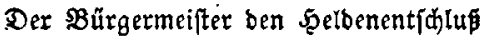

unb fergte fid frade auf ben șintermann

unb biefer wieber auf feinen jobann,

unb Einer fo fort auf ben 2 cnoern im sireile,

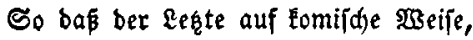

Damit er bod) aud einen Stükpuntt nakm,

Zuf ben $\mathfrak{B}$ ürgermeifter 孔u fiken $\mathfrak{k} a m$;

uno fo pröjentirte bas ganze Ding

Einen Furzen fohrwarzen berweglihen Fing,

Der aljo fíd vorwärtb Eauerte, bas

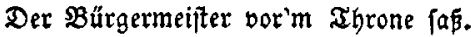

unb nun trug biejer nach $\mathfrak{F}$ ug und Sitte

Dem Rönige yor ber Stabt Drleanz sitte,

unb als bie Rebe zu Enbe lief, 
Da ftanden fie auf und verbeugten (id) tief und (d)wiegen wieber. - -

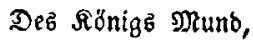

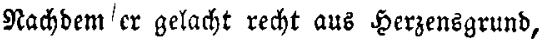
That endlid) Den Rathamännern Folgendeb kund:

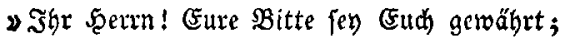
Denn billig ift, was Shar von mix habt begebrt, Beht bin und werfintinet in Eurer Stabt,

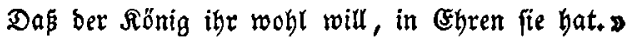
und ald fie zut ahüre fid wanbten barauf, Da bielt er fie nod mit ben $230 r t e n$ auf:

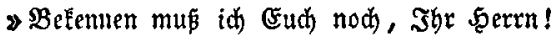

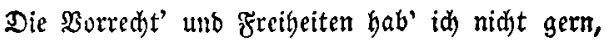
SBefonderb wonn fie fid) grünben auf Sachen, Die lâppif d) fint und nidhts brect)en, nidgte madjen. So glaub' id żum seifpiel: bie Majeftät Berbient, das vor ihr man in Demuth fte $t$; Drum wollt' id audh biefe Belegenheit nükęen,

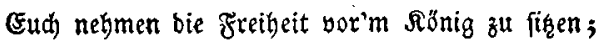
Dod) (eh' id wohl ein, baz geht nidgt fogleich; Denn Shr tragt bie Stühle immer bet (E)d ; (Bebören fie aud) nidit zu ben bequemen, 
322

So Eann (E) boh biefe ber İetfel nidht nebmen;

Drum mag es benn bleiben beim alten Fuf.

Rebt wobt uns bringt Drieans meinen Grup!s

Into als fte nad) Drleans Eamen gurface, Erzăblten fie borten ibr Mígejdice,

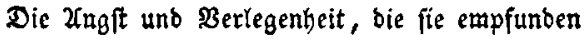
unb vie fie bod glüdtid heraus fid gefundon. Die Staot war ganzer orei Iage lang Beleuditet, uno unter Trompetenklang Berfïndet' ein Serold in allen Baffen, mise fid bie (B) fandtidfaft nidgt nebmen laffen Die uralte Freibeit, bie fie beieffen, und Egrenfetten unb Dankabreffen Potirte man כenen, bie in Befahr 3u fiken verftanden gar rounderbar Un einem Drt, wo fein Seffer war, unb alio für Srleans fpät'ites (Sejhledyt Nit Rlugbeit bebauptet ifre altes Red)t.

3. J. Gaftit. 


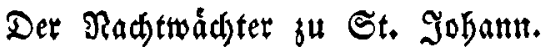

Daß Rcijan fery gar vieles merth,

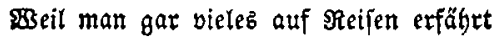
Bon Menjaten und Eänbern und Regiment; Dab man fonft gar nidft erfägrt und Eennt, Dab thät fid felbft zur näd)t'gen Stund' 3u St. Sobann bei $==$ mix Funs.

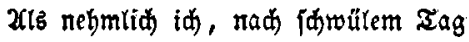

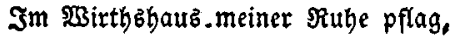
und mir ber wirth erzähtte febr, Trie 2ules beftellt im Stäbtlein wắr, und groper und Eleiner $\Re a$ th babei Mit Bürgerfdaft ganz Einz nur fey; Da fam, faft wie ein 2cbenbftert, Jufż und an bie Eaterne bielt fid gentau Ein Männlein ganz im Mantel grat, unb an bem Mämlein hing nun roieber Ein män̆tig gropes f̧orn bernieber! - 


\section{4}

Dod) hört, eb gebt bab \$orn aud) lob! gaft wie ber großje Irompetenftó, ats Jerido cinft mutete vergebn;

Dod .St. Sobaun, baz blieb ganz ftehn.

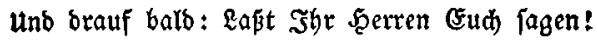
Die Glode bat Gdjlag zehn uhr geidlagen! $\mathfrak{s e r}$ nod) zu thun bat, magz nur thun, ş3er nid)te zu thun, mas jolafen uno tubn!

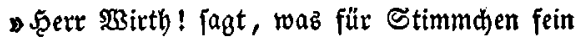
Sat benn bort bas $\Re$ adjtwädterlein?

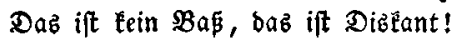
şie ift Daz hier bei (E)d zu Land? So ruft ja kein Rad)twäd)ter $a b ! »$

und fluge ber wirth mir 2untwort gab, Indes er fah red)t liftig brein: 》' 's wirb heute wohl dab Dienbel fenn!s - Dab Dienbel febn! - - bab maht fürwabr Die Sadje mix immer noh nid)t gang klar! 
Drum gebt mir beffer noth Bejdeib;

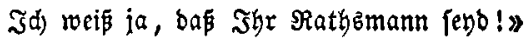

Da wieber rüdrt $\mathfrak{e r}$, baz begriff ih, Die Müs', auf feincm $\mathfrak{K}$ opfe pfiffig, und gab mire nun recht pünctlid an

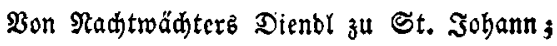

ఎ's war freilid, fprad er, viellcidit eine Finte, Bom Radbtwäd)ter ałz $\mathfrak{B a t e r}$ und fcinem Rinbe,

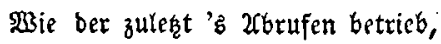
unb mandjmal gar zu baufje blieb, Stod) lang ebe 's frühe war und Morgen;

Sndeffen man will für bie Seinen forgen! - Doth Einmal, als bie ganze Rattit

Der $\mathfrak{B u ̈ r}$ germeifter Gatte gerwadt;

unb Zabends fdon, id glaube von Sicben

Der Nadytroäd)ter, war ganz auzgeblieben;

$\mathfrak{D a}$ gab'b am Rorgen um Reune jotion

Beim gropen uno Eleinen $\Re$ ath Seffion,

\ $\mathfrak{B S}_{\text {ix }}$ fo im Eleinen Rathe, wir maden

Mandimal jo ganz vorn weg bie Sadhen; 
So bas es hernath ein Rcidtes ift, \$Benn's weiter ber grope sath befdlispt. Man fprad gar Biclez hin und her, Warum man aut zu $\Re a t \mathfrak{h}$ aus wăr, und Einige waren ferb/t, bie es trafen, Der Bürgermeifter bătte nid)t gut gejdlafen, Die Pradit hindură; bod weiter bradht' (E⿱ Eeiner, wie er aud nachgebadt. 》

- St3ie aber brauf ber Serr nun Eam und felber bas $\$ 230 r t$ im Stuble nabm unb erb unb 2 á̛n nun exthärt,

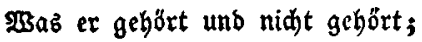
unb baß wir uns ja müsten jăámen, xzenn Frembe nah St. Johann herpämen, und (Einer etwa bie Radit nidt falief uno bann ber sad̆twädter ab nid)t rief: So fabn wir aue felber ein, Dá (Einfebn müpte genommen feyn.»

- Doch baß́ id''s madje furz unb zar, 2Bie bas fith orehte wunberbar; 
Der Rad)truädter Eam unb geftanb ce offen, Wुie bas fid hätte geftern getroffen,

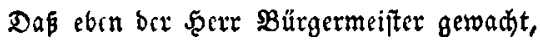
atz cr bie \$runbe nid)t gemadht. Dann that er aber wicber Elagen, Bon Sceiferkeit und f(jwadjer \$ruft uns fagen, Bon $\mathfrak{3 i n d}$ und खBsenn ex Frülymorgens ab nun ruft, und baj cr bod toieder beftefn nidit fömnte, \$3enn man ben Dienft ihm nidgt megr gönnte, und bann bas Dienbl, wir müstenz faften, Ja fetber aud müpte verhungern laffen. Da ging's erft an ein $\Re$ athen unb Sinnen, Was man nur foute nod beginnen, Der Stabt zu belfen und aud bem Mann; Dodh Eeiner war, ber etroas er [ann, »

\ Da trar's faft wie ein 3ufallşpiel, Das felbcr bem Manu etwas einfiel, Daßs woir bann auth, als wirs vernabmen, (Bar nid)t exft zum Botiren Eamen. Er minte nchmlid, wenn er verbliebse 


\section{Des Didterling $\mathfrak{R} \mathfrak{a d c}$.}

Dort über ferne Sartenzäune

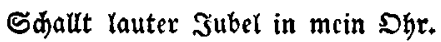

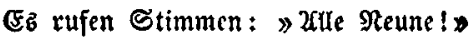
Meint man ber Mujen heil'gen Ehor?

Sit bort ein Sountestag ber Didyter? Эd) lege fdhnell mein fernrohe an; Dod) erzprofaifde (Sifidjtex Erblid' id) auf ber Jegetbahn. Da fälut mir ein, baß idh gelejen,

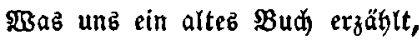
\$zarum man bei bem Negelwejen Die 3abl ber Mnujen hat gerwählt.

Ein junger Şohleopf wollte bidten, uno rief ben Suren: "Stegt mir bei!

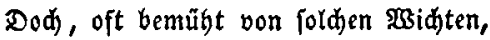
Berjdmälgten fie bas sittgejdrei. (E⿱ fam von einem hohen Dadje, und fifallte bell und grell empor; 
Dod) fie verbüllten fich oab $\mathfrak{D h r}$, unb ogne Beiftand blicb ber Sdyadje.

Er war barob ergrimmt unb jann, 23ie folde 3werglein thun, auf Radje, Dic er gar wunberlid begann. Er ließ von einem Bimmermann

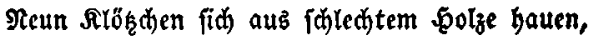
unb malte bann mit eigner ફ̧anb Die Mufen brauf alz abgetebte Zrauen. So ftellt' ex fie auf platten Sanb In abgemesne Reib'n unb Brieber, Rakm in ber Ferne feinen Stand, and rolfte fie mit einer אugel nieber.

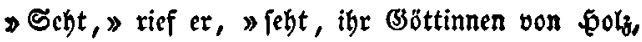
So ftraft ein Didter euren Stolz!p

Bei biefem Rarrenipiele fanben Jhn feiner Freunde brei bib vier, Die mit bes Eorbers Eghrenzire

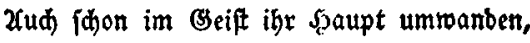
Dbgleid fie eben fo, wie er, 
332

In Keinem günftigen $\mathfrak{B e r k e g r}$

Mit jenen Jeimmelstöddtern ftanden.

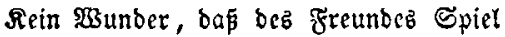

Den jungen Fäntthen roobl gefiel,

und fie fid rafd mit itgm verbanden.

Bald mifdite fid (Beminm

Sie wetteten, wer ueberwinder

Der meiften Marfen würbe feyn,

unb rutrben fo Dez Regelipieto Erffinber.

\&angbein.

\section{$\mathfrak{M} \mathfrak{i} \mathfrak{B} \mathfrak{B} \backslash \mathfrak{a} \mathfrak{d} \mathfrak{a}$.}

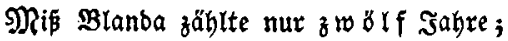

3um Treihgefdent aus Mutterbano

Empfing fie Sidnall' und gurpurband:

Nur renig, bod ein theureb Pfand;

Denn Branda zählte nut zrölf Jahte. 
Dia sianda zählte breizchn Jabre;

$\mathfrak{D a}$ lag ein feiner $\mathfrak{2}($ manad)

3um şeihgefdent im \$puggemad.

Sie jang bie Riebeblieber nad):

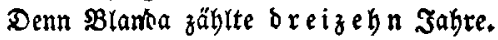

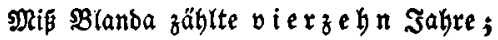

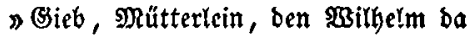

Mir bod) zum $\mathfrak{B r a ̈ u t i g a m ! ~} \mathfrak{D} \mathfrak{j a ! » - ~}$

Mit Spott' im Eädeln iprad Mama:

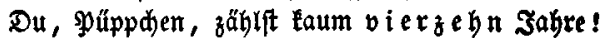

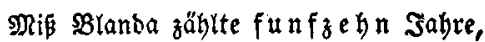

Z(d)! unb crlag bem Liebebgram,

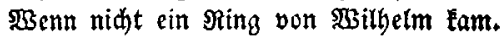

$\mathfrak{D}$ bu beglüđter $\mathfrak{B r a ̈ u t i g a m ! ~}$

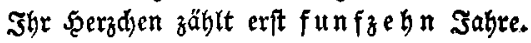

Míp Blanda zählte fe d) zel $\mathfrak{n}$ Sabre;

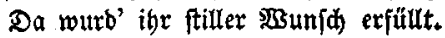

$\mathfrak{Z n}$ ihere $\mathfrak{B r u f t}$ lag Gold und mild,

Ein Inäblein, seiber Ebenbilb.

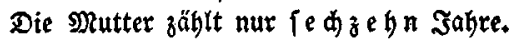

Fra. $\mathfrak{g} \mathfrak{a} \mathbf{u g}$. 


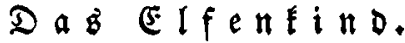

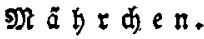

Fïnig Dtfricb fas einjam auf ben 3innen feines

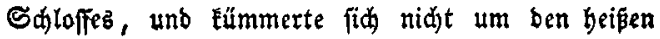
Nittageffrabl, ber auf jeinen Eablen Sdycitel fiel. Sein 2uge ftartte binaub auf bie weiten fjerber zu ben Füpen bea Sdlopberges, auf ben langjam ties felnden \$adt), auf ben entlaubten Eidjenivalb, uno bant fah er brüber weg. auf baz ferne Rebelmeer, auz weldem bie violetten wunberlid geftalteten Fel= fenberge berborragten. Die Dittagêforme brannte

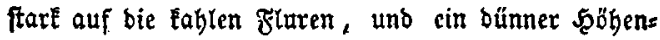
raud) quoll ḧberall aus bem $\$$ Boben empor. Zub bes

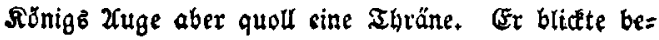
forgt nady allen Seiten umber, benn ihm fdien eb, als gewönne bie ganze todte Ratur ringsum Leben, aber lein fröhlides Reben, fondern als wenn um 
Nittemnt alle (Gräber auf bem Firbgcfe fich aufs thăten uns bie bleiden Ecid)name herausftiegen. Die Dürre (Fide unten am Sdylopportal reclite ibre mie Sdjlangen / oerwunbenen Wefte, und fojien ifm mie ein aus ber Erbe getwadjener goolyp irgend ein lebend

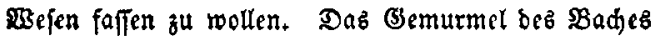
war ein Stöhnen und $\mathfrak{a b c h l a g e n , ~ i n t ~ N e b e l ~ a b e r ~}$ fah er blaffe bagre

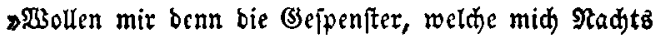
unb in ber Plorgen = unb 206endbämmerung quäten, aud) am bellen SRittag Eeine Rube lafien? Jns Dun= Eel gehören ja bie böfen Geifter. 》

(Ex wifd)te fich bie Thräne auz bem 2lurge, fah mit feftem 2uge rings umber, unb fagte bann: "Эa, es war nur Jäufdung, bie Befpenfter finb fort.

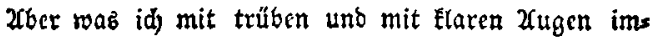

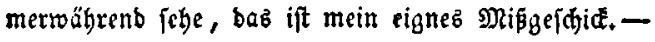
Nidht bie Jahre, ber אummer hat meine b̧aare ges bleidt. Nidjt vom zlter jind meine Blieber fanad), fondern von ber Sorge. Mein $\mathfrak{E}$ eben uno meine \$offinung liegt in ber Bruft bei meinem Siseibe, uno mir ift nidjts geblieben als furdit unb Sdmad, bie 
Feiner meiner Whnen Eannte." So fprad) ber Riönig für fid, , unb fah auf ben alten Barben, ber binter ibm jas. Der aber yatte den Ropf auf bie Şarfe

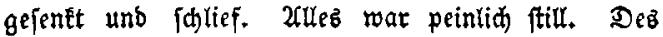

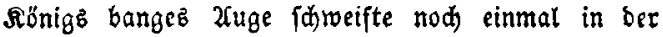
Bsegend umber, Bern hätte er ben Barden gewectit, um bie Şarje anzuftimmen, aber ex wagte es nicht.

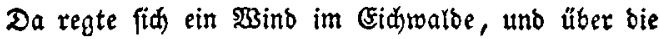
Dctobergaibe jäufelte eह langlam bem Sd)loffe zu, und bie gelben Brashalme beugten fid nach unb nath bis an bie Mautrn bes Ğhlofiez, als wanble eine un= fiditbare Beftait auf ihnen. Flöglid traf ein jomers

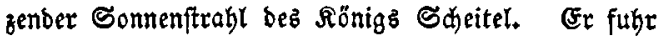
entfeşt auf, und bem Barben fiel bie Sarfe tönend auf Die Steine bes Bobenz.

Zus bem innern Shlophofe, wo bie Frauen unb Zofen auf bem Braje bie Finber wiegten, tönte ein

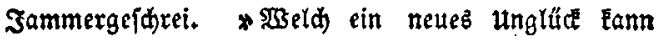
mid) noch treffen? 》 rief ber $\mathfrak{R o ̈ n i g , ~ a l b ~ e i n ~ m i ß g ̧ e = ~}$ ftalteter Snabe mit 5̋̈fern vorn und binten, mit rothen 2 Uugen und Saaren, und äägnen, welde rüf: 
Felartig aus feinem Munbe bortrateh, ungefdict an ibm vorüberiprang, unb ben Bater anläđelte, obcr mehr wie eine Biege anmederte. „Sft nicht johon bas

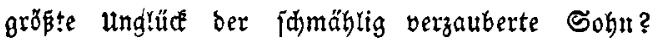
Ift nid)t meine Furdht nod) gröperen unglüc(és tha" rig? \#- Da ftürzte athemloz, mit fliegendem Spaare,

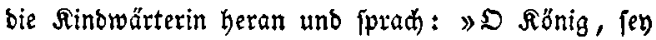
2ulen gnäbig — nod) ein gröper unglüc ift gefdehn.»

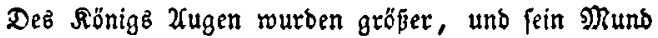
zutute fieberhaft, aber er fonnte nidit fpredien.

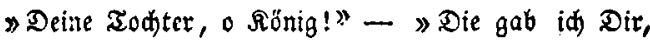
feit ber (Elfe um Mitternad)t meinen Sogn getüst, id gab fie Dir bei erwiger Seligkeit zu hüten. $\mathfrak{D} \mathfrak{u}$

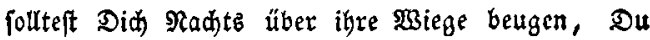
follteft fie balten, daß Bein Elfe ing naben Ë̈nne. Sprid,, — " \$ Rönig" - rief bie W3ärterin 》Deine Iodter 价lief Radts in meinen 2(rmen, uns in ber Mitternadbteftunbe that id kein 2luge zu, feit fie geboren warb. X'Wer bie böjen (Seifter haben aurh Madt zur aagesftunde." - Da griff ber Barbe in bie Saiten und jang aub bem alten Riebe bie $\mathfrak{B e r}\{e$ : 
338

D Menjotientind! jey roblbetadt, 3u Mittag unb zu Mitterna(d)t Şaben bie böjen Geifter Madit.

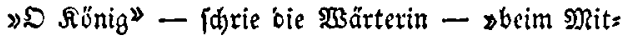

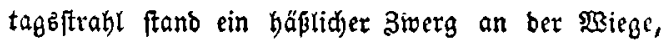
und Eüßte Deine Iodter. ” -

Der Sönig toankte bie Stufen bes ahurmes binat, und fhlug bie gorbgewirlte Dedé von bor 2xiege zurute. Da lag ein ungebcuer in ben Silffien.

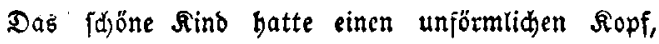
ogne Scaare, tiefe tleine $\mathfrak{H}_{\mathfrak{b}} \mathrm{en}$, und ber Muno ging von einem Dhre bis zum andern. Es buftete un' wimmerte aไb fühle eह ben nahenden Tob. Die

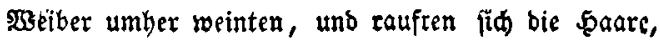
ber Fưnig aber rief aub, nadjeen er lange fdrwei=

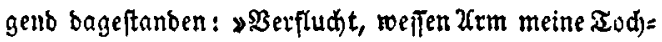

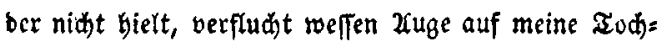
ter nidft fah, berfludt wer bem sroerg nidft abges wehrt, werfludt mein eignes Silberbaar, wenn id bei Mittag ober bei Mittcrnadyt ben frevler lebeno um inith $j a f_{j} ! "-$ 
2ule Beiber fahen fid erftart an, bie Mămner bliáten aur ben $\mathfrak{B o b e n , ~ a b e r ~ b e s ~ \Re o ̈ n i g s ~ S o h n ~ l a d t e ~}$ laut auf über bez Batcra Drohung, „wen trifft mein f(ud)? - rief ber אönig, und bob bie ge= ballte 5 and in bie Rufte. Zfle farwiegen, und zeigs ten auf bes ^önigb Sohn. Endlid fpraden Einige: \Prinz Ebgar trieb bie \$3ärterinnen von ber \$iege, Prinz Fogar miegte bie Sdywefter ein, \$rinz Fogar Iahte ben 3werg an. Der Rönig zitterte, aber fonnte nidt fpreden. Enolid ftammelte ex heraub:

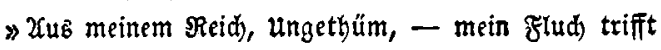

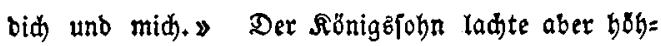

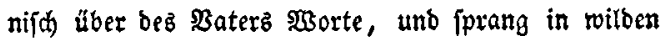
Săkęen über bie bemoofte Rauer, uno fhō wie sin

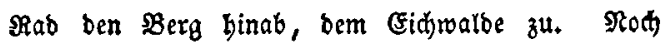
aus weiter Ferne füll jein höhnifdes Gelädter, unb zerrí bie $\mathfrak{B r u f t}$ dez $\mathfrak{B a t e r s . ~}$

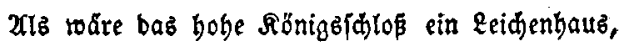
to ftill wurbe es brinnen. Die \$ुeiber verbargen fith in igre sellen, unb bie sänner wagten nidt bem

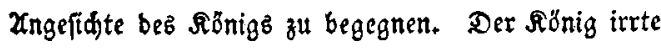
aber wie ein (B)eift burd bie äben Bänge, unb rebcte 
340

âberall bie Seiligenbilder unb Steingeftalten an, weld)e aus ben Nijoen unbl unter ben geroölbten shoven ihm entgegen traten, als wären ez lebende B3ejen. Er forderte von ihnen feinen Bater, unb

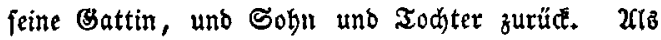
Eeiner ihm antwortete, ftieg er binab in bie Erbgruft, feste fid auf einen fteinernen Garg, uno klopfte auf Den Deckel unb rief: $\gg$ Bater, Bater, ber bu unten 低läfít bitte unten ober oben, too bu bifit, bie Bei= fler, fie follen mix bas sidgt nehmen uns mich blinb madhen, das id̆ fie nidht mehr iehn kann, wie fie ưberall tanzen unb ipringen, uno ben Meniden vers

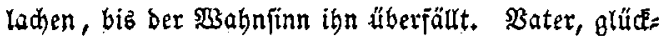
lid) finb bie Slinben, welde aur ben Shein von Zutem jeben, aber benten tönnen. Dein Sobn kann nid)t mebr tadjenken unb finnelt, aber fein 2uige fieht burd Baum und Felien bie unfiatbaren Beis fter. - Şierauf legte er fein Dhr auf ben Sarg= bectel, ftand aber enblid fopfichütterno auf: 》Der arte will nidjt borren, ex wirb nod böje jenn. " -

Urle bie. Sterne am Elaren Simmel ftanden, ftieg Sonig Dtfrieb auf ben hödffen agurm, und als aub 


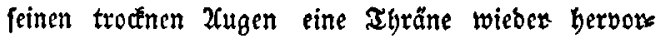
brad), verfdrwanden bie (seifter aub jeinen Blicen, bie Erinnerung fam ihm zurüđ, uno ex iprad):

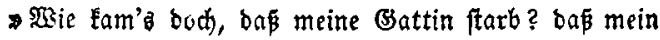
einziger Sohn ein ungeheuer warb, unfähig Sdjwert und Sdjild zu tragen? baß meine Todter verhert ift, unb bas id ganz allein von meinem Şauje als

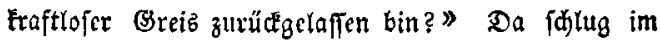
Dunfel bez Scofraumb eine Scarfe an. Ez war bes fïnigs Sănger, und ex jang mit heller Stimme bab Eieb:

গুzas fpornet fo laut burd) Scaibelraut?

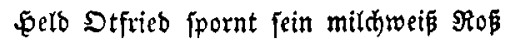
Durdz Elfenthal mit bem jungen (Semahl. Freunblid labet daz Bateridlop Drüben ben Şelden und feinen Benop.

* $D$ bu holbe \&uft an meinex $B$ ruft

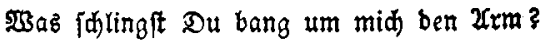
Dort brüben folon glänzt Baters నyron,

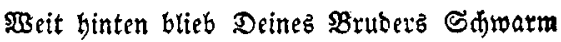
unb bei Dir ift mein Sduett unb 2(rm.》- 
\D fiegft $\mathfrak{D u}$ nidit im Monbentidet

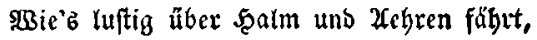
Den Elfentanz im Monbenglanz?

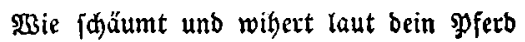

şsenn's burd) bie Tänzerreifen fährt! -

\) Meine holbe Frau! es glänzt ber Thau

Zuf $\mathfrak{G r a b}$ unb $\mathfrak{s a l m}$ im Mondenlidit, unb jpornt ein 5 eld burd) Z Zehrenfeld,

Bcugt fid bie 2 (egr aus foulb'ger spflidt.

Fin seelo fieht nirht ber (Beifter (Sezüut!n-

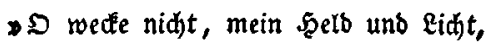
Dac Elfenvolkea 3orn.

Эh) fürchte fie mehr alz $\mathfrak{B r u b e r z}$ Seeer.

Sief, wie fie bangen bidbt verworm

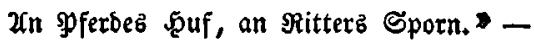

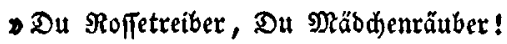

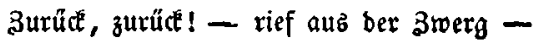

ঙm Mondgeleife, im bunten Rreije,

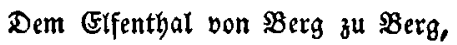
Stört ftrafloz Eeinex ber Elfen \$Bert, - 
》) Sur beiter, reiter - fo ruft ber Reiter -

Whixf ab, mein $\Re o \beta$, ben ahau vum buf.

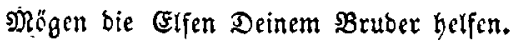

Bom $\mathfrak{B}$ erge tönt jein Geijerer Ruf,

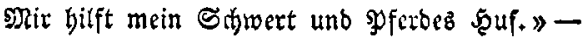

》Du Roffetreiber, $\mathfrak{D} \mathfrak{M}$ Mäsdenrẳuber!

Sthlimmer als Menjd)en = ift Elferzorn. Zest fteht Dein Baul trok Spornen faul.

Dodh fieh id geb' ifm den Elfeniporn,

Denn überall trifft Did) ber Elfenzorn.»

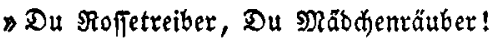

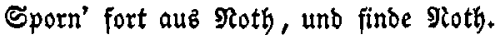
Dein fidön 'Semahl trag' in fidy Duar,

und bringe bem Bater Noth im $\mathfrak{x} 0$;

Denn fejt umftriat ber Exfen (Bebot, ) -

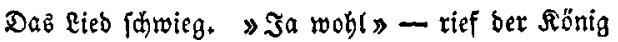

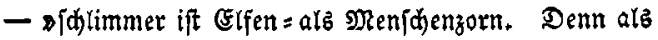

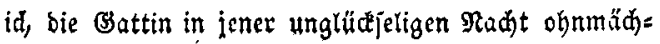

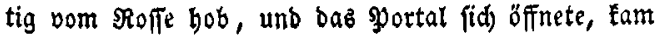
mir bes Baters Eeidengug entbegen. Die Battiı 
(h)ente mix ben Inaben, unb Erfen befdrliden ign um Mitternadht. Ex warb bäplither als ein 3roerg.

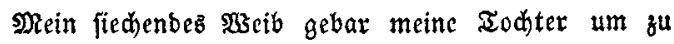
fterben. Mein Sohn verricth bie Sdweiter ben zür= nenben (seiftern, und id verftiés ben mis̈gebornen Sobn. Fun (tebe id) allein, aber bab (3eiftergezülyt, bas id im Elfentbaye nicht fehen woule, umgaufelt mid) juberall, uno id) erblicke bie gefpenftifaten Bsis fter in jebem verwittcrten Stcine, 》

Sn einer Sibludit bes (Eid)watbez, wo bas furdjt=

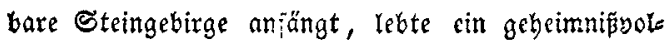
ler Mann. Exr pfligte feitres Menranen umgang, aber bie Sage ging, er verftehe ben Sang ber $\mathfrak{B}$ öget, er unterbalte fich) mit ben'Bäumen, wenn bie Sironen bex Eithen won janftem Zwenshaudje gefdaufelt, ober bom Norbminde gepeitfift würden, ber $\mathfrak{B a d}$ rede immerwährend zu $\mathfrak{u}$ hm, aud folle er jerbft ben leblos fen Steinen Spradhe abgewonnen haben. Sft roolts

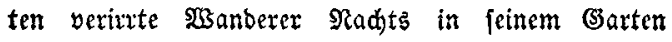
fdwwarze 3roerge und monbweipe (Etfen gefehn baben. 3au biejem ging Rönig Stfried am Morgen, und ers ftaunte nidt wenig, als ex Dez Einfiebleraz fleinen, 
3wiłd)en mooffge Steinmaffen eingettemmten Barten, zux Serbftzeit in Frühlingabrüte erblicte. Zud) fajien bie Sonne hallex bier in bie riefe, gleid als wenn Die Ferfemwände igre Strablen auffingen und ver bopact in ben Grunb Gerobroulefen. Sit fdon batte Rönig Dtfrieb bei bem Siebler Rath erholt, wenn bie Befpenfter um ihn zut mächtig wurben. $2(u \mathfrak{d})$ bette fragte ex zuexft, inocm ox fich exichöpft auf einen Gtein nieberwarf: 》 Gind aud feine Exfen und

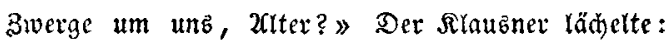
\$ Benn Su nidht willt, follen fie fern breiben. 2 ber füidisteft Du noch immer die freundrid)en (E) (fen? 》- Sie verforgen midh nodi immer fo unerbittlid 》 -

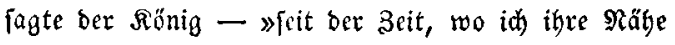
leughete." - Der Greiz erwieberte: "Sieh Darin Liegt $\mathfrak{k}$. Du roolfteft fie nirgends fegcn, barum must Dut fie jest überall erblicken, uno in igrer fürdters liden (seftalt. $\Im$ d) babe fie überall aufpefudt, barum müfien fie mir eridgeinen wie fie fimb, und es ift ferten, bas fie niđjt freuntolich zu mir Eămen, benn fie find alke exidaffen bon unferm serrm und finb feine Diener, $\mathfrak{s h r}$, bie ihk eudi) vor ibnen fürd)tet, 


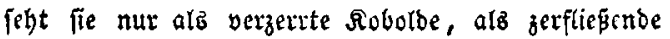
Monbgeftalten, als thierifde ungeheucr, und ihr $\mathfrak{m}$ ü $\mathrm{t}$ fie jefen, wenn ihr audi nidht rollt. Sie ftõren eud in euren frobften 2cugenbliden, fie ums

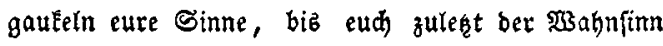
überfällt. Id) aber jehe bie lieben Erfen in jebem 仿onen, grünen Baume, ber feine Xefte zum şims mel aufhebt, ifh fohe fie in bem vom soinde getrie= benen (3erwsle, im herabfallenden welken satte, im WSalbmoos, bas zutü đënkt wenn mein Fus barauf tritt, id fehe fie im grauen (s)antit wie im Babletes fel, benn überall, burh bie ganze Schäpiung ift bas

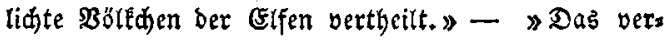
ftebe id nidft, $\gg$ - jagte ber $\mathfrak{A} u$ ung, indem er fein

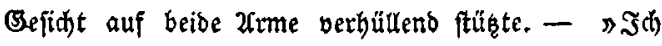
găbe aber mein halbes sünigreid, wenn idf fie nie Befeben hätte, und fie nie mehr erbliden müfite, wie fie mir immer höbnend unb brohend bor ben offnen

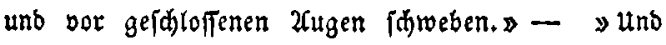
bätteft Du fie bamals gé́ehen, 》 - jagte ber Breis - und bätteft ignen niđjt getroget, - bann blühte

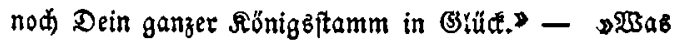


fonnte iff thun" - jagte ber אưnig - wea bcr Bers folger hinter mir war." - \Der Dich yerfolgte, war ein Menid), - aber bie B̧eifter finb überall.»-

Der Sünig erzăglte fein feib, und fragte, wie

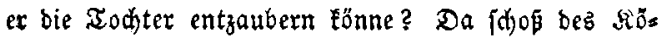
nigs Garke plöglidich auf eine Jaube, pacte fie mit ben Sirallen und hob fie hod in bie \&uft. Soben abcr lick er fie fallen, und fie fant gurrend auf bes

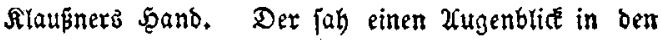

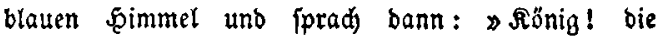
Taube und Dein Fale haben gu mir gefproden:

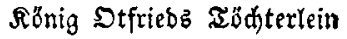

W3itb bald genefen fonn,

Wsem Rünig Dtfriess Sokn

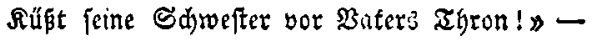

Der Fönig blicte einen Zugenblide farr auf, bann rief er aus: walfo niemalz, benn ich habe meis nen Gohn verflud)t - unb babe midi jelbft verfludht, wenn id) meinen Sohn zurüdrufe. - 2(ber ez fey, lagte ex nadh einigem $\mathfrak{B e j i n n e n , ~} \rightarrow$ maz liegt an meinem vergifteten seben? Zuffudien will id) ben 


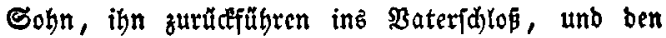

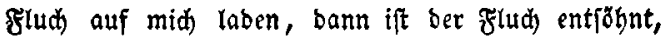
ano meine Ioditex mag auf bem (5rabe bez Baters

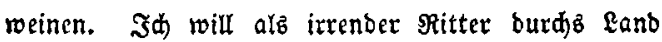
zfehen, bis id ihn finbe, ober im lang entwöhnten Şarnif d) meine (Brieber mobern." - „ Dann nimra biejen Kalismann" - fagte ber Siebler, "mit Dir.

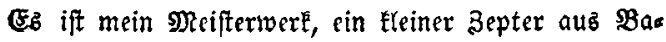
falt, ber Dir bie Madtt giebt, im Elfentanbe bie Beifter zu jehen, fo wie fie vor bem 5̧errn, ibrem Weifter, exidheinen müfien, wenn fie (iid) aud now fo (d)recticlic) verwandelt baben." - Der אönig nabm banlbar bie Gabe, unb eilte yon bannen.

Durdh bas Elfenthal ritt ein gebarnijhter Feis ter. Fs war ber Rönig, welther yon ben Erfen feinen entflobenen Sokn wieber holen wollte. 2lber wie ex aud aus bem gefdrloffenen Bifier ringb umber blickte, er fah Feine Ronokieije, ez Yebte nidytz auf ber tobten Scaibe, unb jein 3elter ftampfte nur bas rothe baibekraut mit bem Şufe. Der Siönig rief

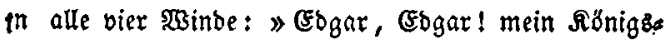
Finb ! aber ber \$ssino nabm bie ş3orte mit, obne 
Untwort fu bringen. Hcberhaupt jah ber foònig

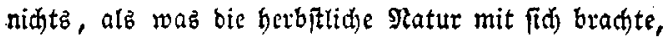
und bie Beipenfter und wilden Geftalten, weldie fonft beftänbiog feinen kranken Sinn umgauketten, flos ben ifgn heut, wo er fie gerabe judbte. 2flo bie Radit anbrad), lagerte ex fid auf reidjem roofe unter einev bürren (Eiche, und gelobte feierlid, ebe

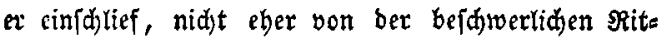
terfahrt abzufteben und wieber als Rönig aufzutro ten, olb bis er feinen Sohn gefunben, ober - æadje genommen am Bolf ber Elffen, flüfterte ihm ein bojer (belft ein. 2fuch im Shlafe nahten ihm keine

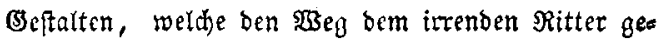
wiejen hätten. Deshalb übertieß ex fíh am Mtorgen ganz ber Eeitung feines \$pjerbez. Diejez führte ign

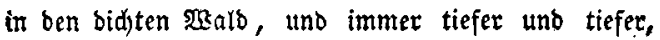
biz Der $\mathfrak{A o ̈ n i g ~ j e l b f t ~ a l l e ~ S p u r ~ u n b ~ \Re i d h t u n g ~ v e r t o s ~}$ ren hatte. So ritt ex einen, zwei und viele rage, obne bá cr ferbft megr wuste, wie lange er geritten fer. Seine Rahrung waren bie wilben şerbjtbeeren, ober et iprad bei einem einjamen $\Re$ I̋hler ein, niemals abew ăbernaditete er anders als in feinem eifernen fleibe, 
und unter freiem bimmel. W3ie ar aker aud fudte, er fanb nirgends Elfon ober 3werge. Dft bob er im Mondenlidte, ober wenn bie Mittagejonne grab auf bie sucllen fotien, feinen Bepter in bie 5̧öbe,

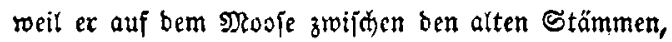
ober im Rauthe ber Suelien auf ben grauen Steis nen Beftalten zu fehen glaubte, aber alles verfdirand, unb ex fah, baßj et fid getăujät habe. Der Rïnig glaubte, er müffe fifon mehrere Monate auf feiner Fahrt jeyn, aber bem wiberfprach, baj er burdjaus

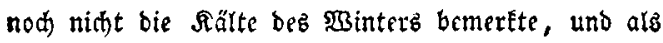
ex eines Morgens auf ciner höhern Felepplatte ers wathte, erblicte er im Morgenbuft bie Igüren jcis nez Sd)loffes. Ere Eonnte alio nod) nitht weit gerits ten feun. Dod fdjien es itym zuweiten, als reite ober übernadte er auf wohlbefannten Stellen. Eines

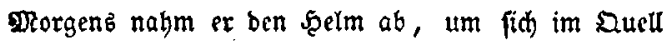
ou wajhen. 2⿰r子 ex fich) aber hinübcrbücte, erftaunte

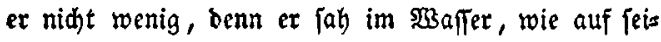
nem gebräunten (Befidte ein sBart von rocnigftens fedhs Monaten ftehe. 3ugleid) hörte ex bie Böget luftig in ben sareigen fingen. Er blicte auf, unb 


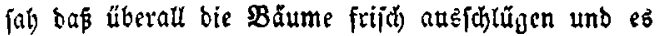
Erüłling joi.

Sun merte er wohl, baß bie Bsifter ihn itx und immer in Rrcije mifíten umbergefügrt Gaber. Er bejhlos bezhalb in graber Riditung yon feinem Salofte in baz Steingebirge einzubringen. Dic Fct= fen fojienen to ftarr zu feyn, und fo ununterbrod)ene Bäänbe, $b a \beta$ ex nur burd)bringen köme, wenn cr mit QRauerbredjern brauf loß̈ginge. Wher es war nur Gidcin. Denn wenn er näher fam, uno crmftlid fudte, fand fid immer eine Spalte, burd welche

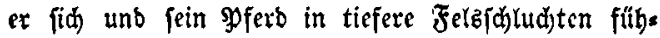
ren Eonnte. Er Eam in bie wunberbarften Gegenben, aber nirgende fah ober börte er (Seifter. Enotid) fprang er mit feinem 3citer von einer beträdtlid)en Wöbe in einen tiefen Felfenfeffer. Wuf rötgriden

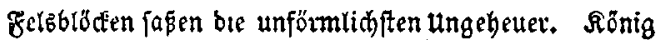
Dtfrieb hob jeinen Stab auf, uno rief: yseifter, wers bet was ity jeyb! unb augenbliffich verroanbelten fie fich in bâplide 3werge, und mitten unter innen \{aß ber bäplidjite - Säntg Dtfriebz Sohn. Der

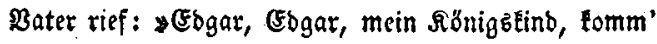


352

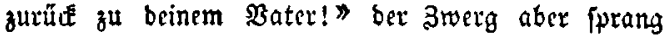
ftatt beffen höbnif ladjend auf einen böbern Fels. Der Aönig ihm nath, Der 3roerg nod höher. Der Ritter mufte vom şferbe fpringen, Fletterte inm aber

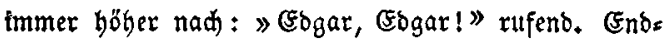
Iid, als Eein högerex Stein zu finben war, fdrwoll Ebgars Beftalt zur unförmlidien Bröß̈pe an, uno Rönig Difrieb erblickte nixts um fid alb biefe fortect=

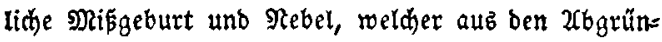

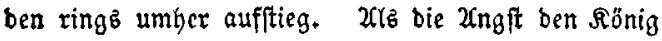
(d)windeln madte, grif ex nad) feinem Stabe unb rief: 》 Seifter! werbet, rab ihr ichb!" und augen= blidilid jank ber Rebel, Gogar jđrumpite zum 3werge ein, und ftürz̧te fich rüd'lings in ben 2rbgrund, aber immerwährenb, bis jeine Stimme in ber tiefften riefe berfallte, rief er bem Sater zu: "Nie fomm' id willig, nie fängit $\mathfrak{D}$ u mid.)" Die Jabenojonne bes leudtete die wilbe Begent und bie mit rotgem 5 aja Dekraut bewadjenen Felakuppen ringzumber, unb jestt

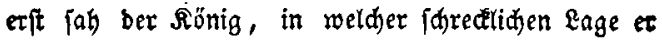
fich) befanb. Er ftand auf einer einjamen Jels̄pizize,

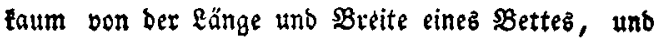


alle andere Felsfpiąen rogten nur tief unter ihm aus bem Nebel cmpor. Der Wbend madte eb igm un= möglid) ben Serunterweg zu finden, und je öfter er

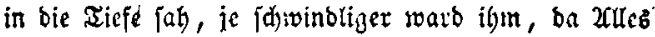
unten Meer und $\mathfrak{B}$ tandung, und bie Felien fid in einanber zu fdjagen fójienen. (Erft als ex ben Stab ergriff, unb rief: "(Geijter, werbet was ige fenb!" watb afilez rubig, er aber muste bas trautigite unb gefäbrtidjfte Lager auf ber Felbiappe sinnehmen.

Ula ber Morgenfrahl ign wedite, war bie (3es geno weit freunblither, obgleid bie ftarren Felekegel nod) immer eine fdredflide satur vertünbeten. (Er wijote bie 2fugen auz, und freute fich ber weiten Xusfidje über baz Steingebirge, aber in wciter Ferne mufte er wieber erblicken - jein cigenez Sdlop. Nur mit Rebensigefahr ftieg er in ben tiefen (Grunb binab. Zuls er aber unten fein \$pert judte, fanb ex wohl ben saum, an reldien er eb gebunben, aud) noh) ben 3aum; fatt bez $\mathfrak{x}$ gieres felbft aber, lag nidht weit bavon ein verbleidteb $\$$ perbegerippe, uno Raubvögel flatterten barüber. Er blicte fid) ets ftaunt um, unb fah baß abcrmalz Frühling war. 
Sben auf bem Elfenfteine mufte er melyere Mronate

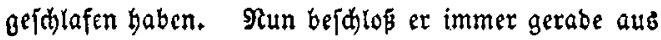
zu gehen, unb unverwandt feine Zufmertfameit auf bie gerabe Ridhtung, welde ihn von jeinem Sdloffe entfernte, zu ridten. (Er flimmte mit megr alb

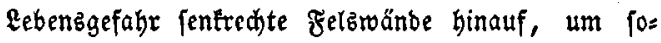
gleid) auf ber andern Seite wieber fid binunterzu= fenter. Jmmer finfterer, immer jarectidider wurben bie Sdjludten, bals fanb er fein lebendes balb Eeine झflanze mehr, unb bie Fetzrände waren fo hod, baß es an hellen rage fo buntel wie am norbijaen Rovemberabende war. Nur bie raujden= ben Ruellen zetgten, baß bie Ratur nidjt ganz ver= fteinert jey, benn felbjt bie suft bervegte fid nidht.

Stad langem 2 zanbern preste er (id) burd) eine enge Spalte, und trat nun in einen großen Feliens jaal, bergleiden ex nod) nidt gejehen hatte. Steile,

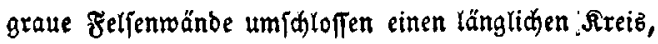
unb extoben fid bis hod in ben Şimmel, fo bas man ihre Spiz̧en nidht mehr exkennen modte. Dben wölbte (id) nur ber forizont über ben Saal, bena nod) war es wegen ber böke fo finfter, bas man 


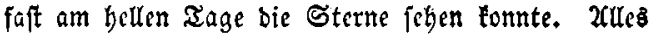

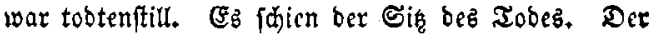
Sönig jah nidjtz, abex cben im Nidjts afnete ex bie Beifter. Gr bob ben Stab unb rief: D Beifter, wers bet was ihr fend!" und augenblictlid warb eb helle wie von Monblidyt und Sdjwefelfammen, und ber SBins faufte furdtbar unter ber Erbe uno in ben

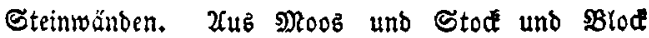
fprangen frofdartige (snomen, 3werge arbeiteten fith aus ber Froe unb ben Felfen, Elfen fanebten aus ben auellen, überäll warb ez lebenbig, uno alle Geifter ftulten fich um ben gropen Bajalttyron, auf

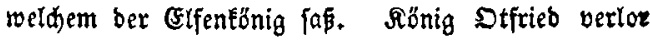
nidjt ben Muth, er zog fein. Sdroert, unb fprang

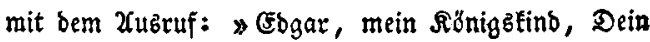
Bater ruft! auf ben 3hron zu, und woltte feinen

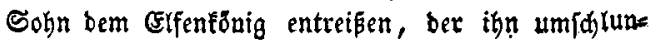
gen bielt. Die Iruggeftalten exidreckiten inn nid̆t, fein Sdwert bieb nur burdh \&uft; als er jebod auf bem IEhronjeffel ftand, war bas Elfenvolk ver/dwun ben, unb ex fabe nidhts, alb wie fein Ebgar bodoe ben auf einem Felien ftant und außrief : NRie fomm' 
id) willig, nie fängft $\mathfrak{D u}$ miă)." - Dann veridmand

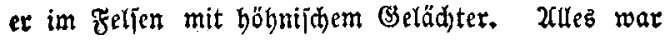
mit ihm veridyunden, ef war wieber natürlide

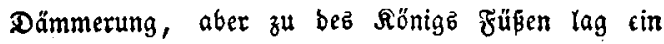

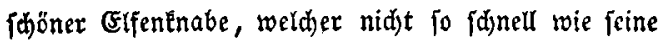
Brüber entflieben tônnen, unb yon bes నönigg בau= ber gefeffert blieb. Sönig Otfries exinnerte fid), ihn

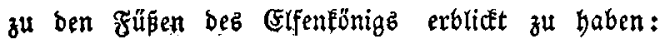
》 Şa!" rief er, 》bift Du bes Elfentönigz Sohn, To fouft Da, senn Did Eijen verrounden ober Feuer

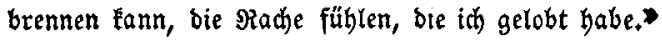
Rund um ladte eb laut auf. Der Rönig aber band unbejorgt bes foünonen אnaben Şänbe, wie aud) ber Ânabe ihn fo hold und gut anblidte, baß ihm das Sherz in Reibe webe that, und floh mit feinem (Ge= fangenen ben Seifterjaal.

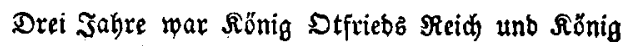
Dtfriebs Burg ohne Serren gewejen, jeģt jaj bes Rönig wieber auf feinem Tagrone, unb bor bem ŢGrone ftand bes gefangene Elfentind, und neben ihm ein Saherge mit bem Beile. Sanft lähelnd blickte

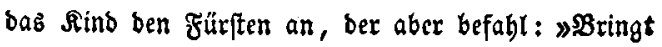


Get meine Todhter, und bann foll ex ftetben.” Die \$3örterin fübrte bab Rir: heran. Faum fah es ber Sinabe, fo berzte und füpte er bas Eleine Mäbden.

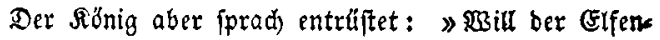
fobn nod mefr ungcil meinem Saalle bringen? Sderge, Dein $\mathfrak{U}_{\mathrm{mt}}$ !» - Der hob bas Beil, ba

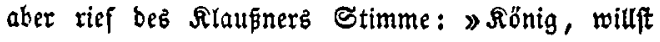
Du Dein eigenes fino morben?" - $\mathfrak{D e m}$ fionnige flohen bie bäplidien Beifter nod) einmal yor ben Xugen vorüber. Uber er rief: 》 Bseifter, werbet was ifhr fend!" und num jah ex alles lidjt unb Gell,

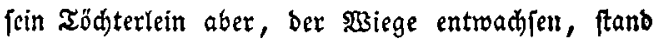

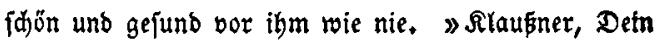
Bauberiprudi war falid)!" rief ber Rönig. ” Metn Sauberiprudi ift wahr! benn nur von ikres srubers

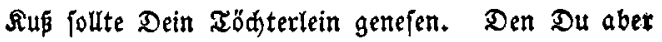
verbannt haft, war ber Elfeniohn. \$shn batten in jener Mitternaht bie zürnenden (Elfen in Ebgars W3iege gelegt und Dein Rönigzkind bafür geraubt. Der TBedjelbal ift nur verbannt, aber Dein Sogn Ebgar ftebt yor Dir. Die Funbe haben bie Duellen 
358

uno Bögel mir getradt." - Der §önig umarmte

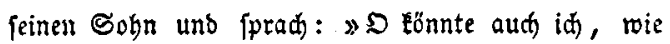
Du, bie Spradie der Ruellen und $\mathfrak{B o g h e l ~ v e r f t r b n , ~}$ wenn fie (Sutes fpreden, 》

şitlibalb atexis.

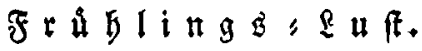 Seftine.}

Was tönt jo jubilirens burd) bie füfte? wex wirbelt fahmetternd frettigige Bejänge,

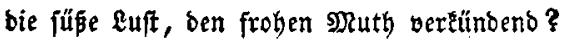

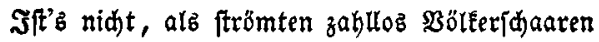
baher aus unbetannten (s)ไültez = Fernen ? - Der Frühling ift erradht" - bie Eerden fingen!

Sein ફerols ift's; ifn friert siefes Singen. Es fäđheln warmen şaud bie netten Rüfte,

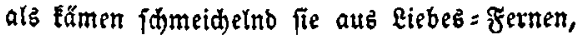


uno buthlten fojent um bie $\mathfrak{R}_{\text {uft }}$ = Befänge, und trügen hoch im Raum bie frohen Sd)aaren ringsum 》 ber Frübling if erwadyt 》 verküntent.

Ibr Kugen foaut - fo bell unb glanzgoerkünbend ben Baum in Rnofpenprad)t; $e$ b tốnt cin Singen aus jeinen 3łoeigen; bunter Blüthen Shaaren ihn fröblich) fdmürexend, treten an bie eåfte, unb fhcinen aufzuhorden bie Bejänge: \#Dar Frübling ift erwadst in allen fernen."

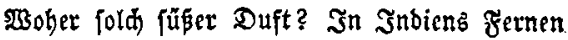
nur Geimi id), bort'ge Sonnenglut verkünbend? Er folgte roobl bem 3auber ber Seränge benn munberbare אraft befígt baz Singen. Beraufकend fint erfüllt bon ihm bie Rüfte: \Der Frügling ift erwadt mit feinen Styaaten, 》

Ja! neucs \&eben quillt in üppgen Sdhaaren,

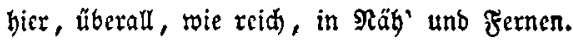

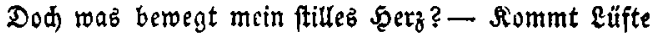

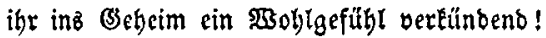


360

Ein rocides Gehnen ftromet aHb im Singen : D Der Frügling ift erwadt unb bie Sejänge.

Setน uns! erneut entftrōmen bie Gefănge. Berjammelt find ber Didter frohe Sdjaaren, ber Lieber Freunbe fint vercint zum Singen, bie $\mathfrak{B}$ tüthen grüßen fie, sie Düft' aub ซ̌ernen, bie $\mathfrak{B o ̈ g e l ~ E o m m e n ~ i h r e n ~} \Re$ hhm vertúndend, weit hallen: " Frühling ift erwadht!" bie Rüfte.

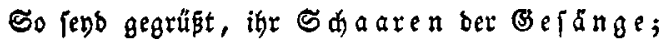
aนb Durd) hetle \&

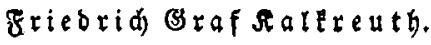




\section{$\mathfrak{D a b}$ Jดbannis 1 Reld Dex Rofe.}

W̉eld ein $\mathfrak{B s u n b e r ~ h u ̈ l l t ~ b e r ~ \Re o j e ~}$ Bartes Roth in Flammen sin? Barg in ihrem horben Shocke Sid bes abendrotges Sdein?

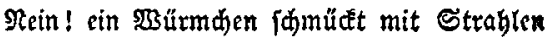

Sein erwähltes \$̧urpur = 5̧aut, und ben field ber Rofe mahlen Btänzeno lid)te Flammen auz. క̧wiץden Raubgehängen đdjeinet

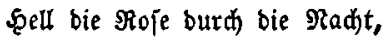
unt fein Fürftentyron vereinet Solde tounberbare sorad)t.

Srog ber

In ber ftermenbellen (S) Iuth

Sthmület er wie mit soealen Seine Scimath, wo er rubt. 
362

Denuod ba, too cr fein $\mathfrak{a}$ ben, In bie Spurpurquelle taudit, Shat fie ibm fid bingegeben, Shren fdỏnften Duft verhaudst. Mag nun hill bie Rofe fdimmern

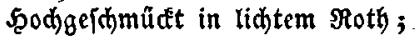
Dod) ifhe $\mathfrak{B z e j e n ~ b a r g ~ i n ~ T r u ̈ m m e r n ~}$ Eignen Dafeyn'b, ffon ben నob. Sell ourd)glübt von Riebebflammen Strahlt ber $\Re$ oje gleich bab Şerz, Dod) mit lidtem Şdmude' zujammen SOB eilt in tiefer Bruft ber Sdymerz.

Durd) bie Nadt bez Eebenz frablen Nag ein §erz, bas Liebe fứlt; aber fennit bu aud ber sualen Xd) to famerzlid helles silb?

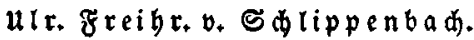




\section{Det frảbling.}

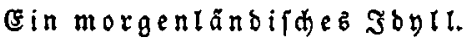

Şorb fingt bie Radtigall, ber Riebe jüpe 3cit Der Frưfling, jung unb juön, finkt atı̇ bem Diften nieber;

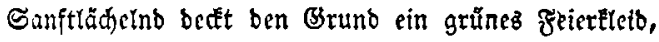
Bon Sitberblüthen glänz̆t bes Mandelgainz Eefieber. Freut euch bes Früblings, bex Ruft gerveiht, 2(i), $\mathfrak{x}$ brüht nur furze zeit!

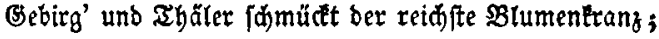
Des Shattenz Milbe wirb bem Bufd zurüdgegeben, uns wöltbend ein Bezelt. Xuf, zum Gefang, zum Sanz!

\$3er von uns resis, of wir ben Frübling überleben? Freut eud bez Frühlingz, ber $\mathfrak{R}$ uft geroint, 2W, cr blüht nur Eurze 3eit!

D פract)t, morin bie Tulp' unb Xnemone fte' 'n, ßorin füsbuftend $\Re$ of' und Rilie fid) wiegen! 
364

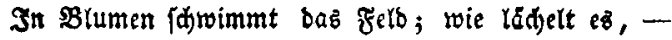
wie โdjön!

Ein Feft fey bicjer Iag ber Frcundidaft, bem $\mathfrak{B r r}=$ gnügen.

Freut euch bes Frũhlings, ber Euft getveiłt, Xd), ex blüht nur f̂turge acit.

Die Ro[engănge bort erglüg'n im Sonnentidit;

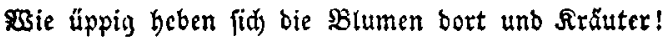

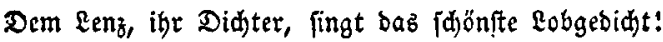
uno ibr, in Gram verlenkt, erbebt ben slite, feno heiter!

Ereut eud be Fräblings, ber Euft geweigt, Wha, et blüht nur Eurze 3cit!

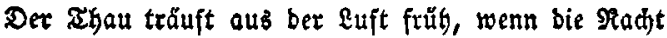
entflieht,

Jn Silber glänzt bie $2 u^{\prime}$, two peine perten fdrweben. Die Rilie, bie Roj' und jebe Blume zieht Xus jeines Baljams Ruell ein junges, frijđę Freut eud bez Frühlings, ber Eupt gewsibt, In, er blügt nur turze Zeit ! 
D Eiebditen! Eitien unb zalte Rofen glüg'n

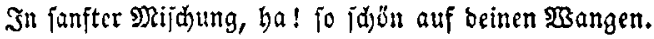

Dod) täu(d)e Did) nur nid)t, wie berrtid fie aud

$$
\text { blüb'n; }
$$

Zuth) inge \$radt trifft einft bas

$$
\text { gangen! }
$$

rFreut euđ bez Frühlingz, ber fuft geroeift, $\mathfrak{d}$ d, ex blübt nur Eurze aeit!

(Ergeb', O Mefigi! ben Frühling Durd bein Lieb,

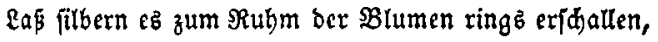
unb too im $\mathfrak{R a ̈ b d}$ enErets man Did - bie Sdönfte,

$$
\text { fieht, }
$$

Da flinge bein Gejang gleid) bem ber Nadtigallen. Freut eud bes Früblings, ber euft gerweitt, 2rd, er blügt nur Eurze acit!

Э. (5). 5․ Bittermann. 


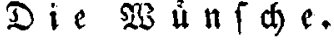 Zeen mährden.}

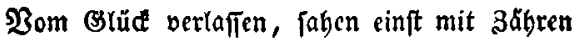
Zwei holbe Sdjweftern in bie 3ufunft bin; Da jant herab ber Feen אünigin, Dit milbex $\mathfrak{p u t b} \mathfrak{i b r} \mathfrak{3} 3$ ünịden zu gewăgrer,

Dap jenes butttben tief in Iyalebmitte, Befairmt vom Blüthengain, ihr eigen jey; (sin ftilles

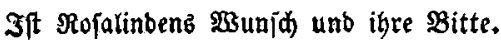

Run wănjdt Rupine, wenn ifr beiß $\mathfrak{B}$ erlangen Dereinft gebeut, in jebez 5̧erz zu \{ch'n, um bab verborgne Sebnen zu eripäh'n; Sie wunnjdt mit Reidhthum, פradht unb Reij ju prangen,

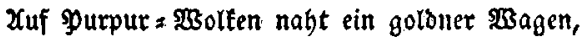
Besiert mit goerten, nimmt Eupinen hin; 
Bon Bitanj umleuthtet fleht ber Stolzen Sinn,

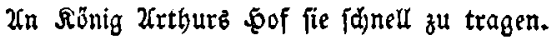

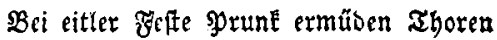
Eupinen balb mit faber äärtlid) Éeit, Die Einem bort nur wahre Riebe weigt, Den fie vor allen Unbern fidj erfobren -

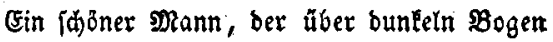
Uuf off'ner glatter Stirne Solyeit trug Bom eblen milten Ernft in jebem 3ug

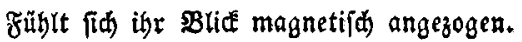

Ex nahet ihx, fie bört mit fanftem ane um Begentiebe ben Erifhnten fleh'n.

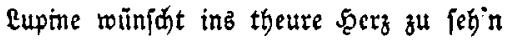
unb wähnt, baß bier ige cignes Bilbni wo wahe.

Sie fieg't unb bebt; wie wirb ithe 2uge tribe Ez bat ber (Sram bez $2 \mathfrak{c m e n} \mathfrak{B r u f t}$ geprept. Sie fieht ben אampf, ber feine ærange näpt,

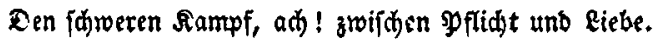


Rtaine niatt, o nein! nur Sabriele

S3ar bier fein Iroft, bes $\mathfrak{E} e$ bens bödftes (Sut,

Da banb ben Bater böjer Zaubrer $\mathfrak{B}$ Zuth

In eine furdbtbar tiefe Gallangenhöle.

Das 3auberbant witb bann nur losgebunben, गुbenn er bem Theu'riten, was ex hat, entiagt. unb einer 2 noern heipe Riebe flagt, Die nimmer nod fein treue şer empfunben.

Stolz, Giferjucht, getränlter Riebe Reiben

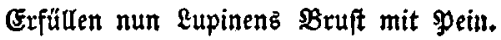

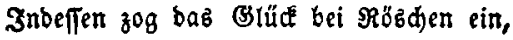
uno jeber $\mathfrak{Z} a g$ gewähte nete Freuben.

Şye $\mathfrak{B u f e n}$ hegt Eein beftigeb Berlangen;

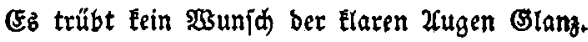
Genügfamkeit! fie kennt bein Blüđ fo ganz, Du mablteft bell bie blüb'nden $\Re$ ojen= Bangen!

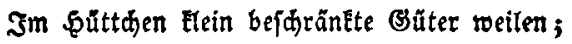
Bejuăftigung verleib't ibr Geitern Sinn, 


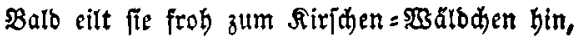
Bergípmeinnid)t uno Frühte mitzutheilen.

Dann roiegkn jie ber $\mathfrak{B o ̈ g e l ~ M e l o b i e e n ~}$ Ifm Murmelbad) in filísen Sdulunmer ein; Sie faläft fo fanft, von ecibenfdaften rein Sinb bimmlija jăôn ber Iräume phantafieen.

Eupine finEt, mit ungefitiftem Sebnen,

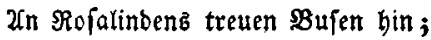
Da nah'te jid) ber Feen Rönigin

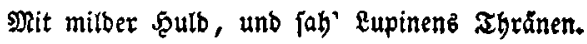

》 $D$ !" bittet fie, 》 Du wolleft einb mir geben, ( Rimm 2uleb hier, was idh fonft bat, zurtie.)

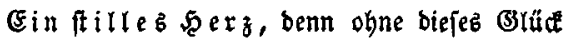

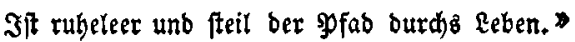

șilgelmine $\mathfrak{R} a$ l. 


\section{Die $\mathfrak{b l a t} \mathfrak{i}$ e Der Erigkeit.}

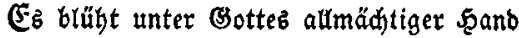

In bes șeltallz unendlidem Sarten

Eine Brume z eitro z von Engern genannt,

Dic bieץe nur pflegen und warten.

Die Brüthe entleimte ber Erwigkeit, Zutd) wird fie nimmer vergegen;

Shy Duft nur allein Eann, alb flüd)tige seit,

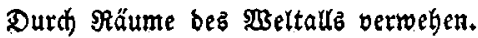

um biefer $\mathfrak{B l u ̈ t}$ the fo lieblid)en Duft Siehft Sdmetterlinge ou foweben;

Sie atbmen bex Blume bal[amijd)e \&uft

und Ëönen nidjt obne fie leben.

Sie baben in engem geidlofienen Bon wed felndem Dafenn burdibrungen, In Zarben ber $\mathfrak{R} a d t$, und bals fonnig und weiß, Sidi feft an einanber gejd)lungen. 
Fn immer glcidjer, nie wanbelnber $3 a b l$ -

Bald naten fie, finten bann nieber,

uno einer nur weilct baz einemal,

Dann febret ein anderer wieber.

In Sd)metterlingsflügch fo zart gebaut

Bon eilendem gluge entfaltet,

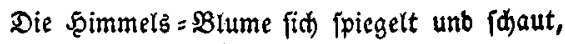

şie bod uno bebr fie geftaltet.

So fdrmeben fie nun voll Şerrlidjeit,

SBie fie fid beifammen gefunben

um die zeitloje $\mathfrak{B}$ lüthe ber Evigleit,

Die flüdtigen eilenten Stunden.

utr, Freigr, b, Sdlippenbat. 
Scab oft mit bir gefprod)en, Dir mand)en (srup gefdict, uno eben ohne Poden รn's 2luge bir gebrictit; Şab' oft mit beinem Sdymurete Bebanécnlos gefpielt, Şab' oft beim Scändebrưde Nidjts als ben Druce gefühlt:

Nun, feit bu fortgegangen Scat fid das Blatt gewanbt, Sid zieht tin jüß' $\mathfrak{B e r l a n g e n ~}$ $3 \mathfrak{u}$ beiner lieben Şand. Beb'n Rieber woltt' idh wagen Für einen $\mathfrak{L}$ aut von bir : Ein Ring, von bir getragen, Ein Âtcinod wär' er mir. 
Tun ift bein Blice mix theuer, Nun büntt er erft mix (Slut: Ex war ein fdyreidjend fouer, unb zünbet jpät, bod gut. Der (Brub bei beinem Sdeiben Durdfubr mid), soie ein Strabl, sit niegetannten Freuben, Mit niegeEannter Rual.

WBo bift bu bingeflogen, Du baft mir'z nidjt befannt. \$230 bifi bu hingezogen?

O nenne mir baz Eanb! Das Rans, - fo wabr id lebe Das Land ift mir berwaft: unb wern'z kein anb'res gäbe, So wắr' $\mathfrak{e}$ - meine $\mathfrak{B r u f t}$ ! Jobann Gabr. Eciol. 


\section{Scer und iofe.}

Alb id̆ am leģten Maientag

Jm Sdatten einer Eidhe lag,

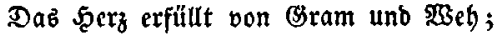

Siam froh auf grïner \$Bergezböb'

Mein trautez Rieb' gegangen;

Dab nupbraun fribne Şar

Sielt weid) ein Irranz tmfangen

$\mathfrak{B o n}$ Rojen jomigetar.

Es grüpte mid fo traulid milo Das Golbe liebe Engerbilb, uno rief mir zu: Sieh hier den Rranz, umwogt von Duft und Sonnenglanz. Sell wie bie Ro广en blühen, In munt'ver Früblingsluft, Jühl iđh baz șerz erglüben Für bid) in meiner $B$ ruft. 
Saum watb aus ihrem Spurpurmuno, Mir $D$ ! bie fitpe Deutung Fund, Wax aud) bes fiummers böfer Gohn, Der finftre unmuth fidnell entflohn.

Dab Brlüc hatt' id gefunden Xuf 'luft'ger $\mathfrak{B e r g e z h}$ b̆h';

Die Sorge war ent/dwwnden, Erftorben jebes $23 e$.

Sdyon ift ein flüdtig Jahr vorbei, unb wieber ift es leģter Mai, Dab arme Şerz von neutem idjwer, Dab Leben trübe um mid her. Mein Lieb' Fommt niabt gegangen Mein $\mathfrak{R}_{\mathfrak{i e b}}$ ' liegt auf ber $\mathfrak{B a b r} \mathfrak{r}^{\prime}$ und weife $\Re$ ofen prangen Im nußbraun feibnen şaar.

Die rothen Rojen find verglüht, Dod) Riebe übers (3rab nod blüht Die Rofen wurben weiß wie Sdnnee,

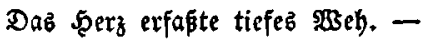


376

Dem harten Sdhidéałbloore,

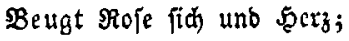

Es bridit ein Sturm bie Roje, Dab berz - ber Iremung Sdhmerz!

- Iheophania.

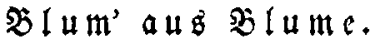

Eeht, wie bie Roje zu ber frühlingstreibe

So lieblid blüht, fo zart und mirb!

unb wie an ifrem Stamm bald eine reue,

Nidjt minber [ajön, bex אinofp' entquillt!

Bro finbet bon ber Roje fid im Sreife

Der Blumenwelt ein Segenftǘd?

Spielt aud Ratur auf nod fo mandje 3 seife,

uno wedjelt taujenofach den silid.

Der Frau allein, ber Eroe fđŏniter SBlume,

\$arb gleide 3auberkaft vertiebn, 
llad jebcm Räbden, bem im Seiligthume

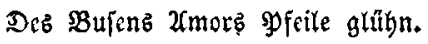

Şa! neue siumen tragen fie, die 5olben,

şsenn ifnen füpe Ricbe ladit,

Rod) reizenber, als aller Blúthendolden,

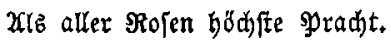

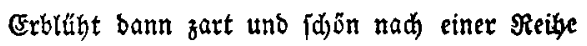

Bon Iahren, burah Dionenb ફ̧and,

2ue jeber Situme wieber eine neute,

So bildet fith ein Rojenband;

Ein Ro/enbant, bab Săuglinge unb B̈rcife

Dit gleider 3aubermadjt um[d)lingt,

und auf ber furzen, lalten kebenbreije

unz einen ew'gen frühling bringt.

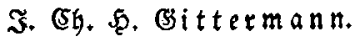




\section{$D \mathfrak{i}$ Bi $\mathfrak{i} \mathfrak{i} \mathfrak{l} \mathfrak{e} \mathfrak{n}$.}

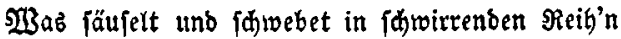

unb zirpt mit gerd)wungenem $\mathfrak{F}(u ̈ g e t$ ?

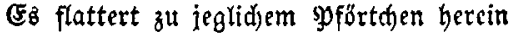

und adtet nidht (Bitter, nicht $\Re$ riegel.

şie Meblthau bie blühenden 3weige verheert,

Dex Bohrwurm bie feimenbe Roje burdfägrt,

So niftet baz graue Befdroaber fin ein

uno zebrt an ber f̧offnung ergrünendem șain.

Dod) balb, allb bez Scaines tief innerftem Shoos, In freunblidfer Stenien $\mathfrak{y f l e g e , ~}$ Da windet ein idwellendes Jeimdien fid log, und fpropt aus bem niebern Bebege. Şoch raget Dionens geroeibeter Baum, Durchouftet mit üppigen Blüthen ben $\Re a u m$,

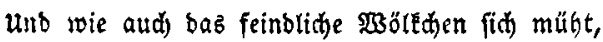
Die Mnrte nur idöner, nur berrlider blüht. 
und fdundl, aus ben filbernen Relden berbor,

Beivappnet, in rüftiger. Eile

Steigt 2Mmors berbrüberter, mutbiger Bgor

und fenbet bie furdhtbaren speile.

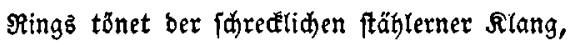

Ring flieben die Erillen in fluthendent Srang;

(5) meibet daz graue Gezüdyte ben Sain, Do thronet nur Riebe, die liebe allein.

SBa ilbelmb. Stubnib.

\section{Die Preife: Pertbeilung.}

(Zrei, nad) einem alten franzöfifăen Riebe.)

J゙ં bat bie Sdonfte junger Sdyönen:

》Berohne bod mein langes Sebnen,

Mein treues Serz, bas feurig lobt!" -

Die Şolde iprah mit 3aubertönen:

> Borerft erfülle mein Bebot

Mem tamft Du Maja's Rindet hier 
980

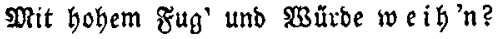

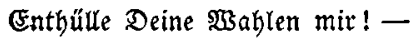

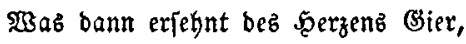

Seb bein, o mein Seliebter, bein!

Sd)on beutet, bas id'b flug bebädte, Kuf ben Slivenzto eig bie Fedhte,

Des Friebent heiligez Eymbol.

$\mathcal{D}$ Dás ez fortan blüben módte

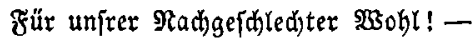

Dir, ben zum Eiebling Fama kübrt,

Der unz ben Frieben fduf, gebührt

Der beilverkünoung $\mathfrak{B i l b}$ allein.

Dein, ber fo vätertid) regiert,

Dein ift Der Selziveig, $\mathfrak{A} \not ̈ \mathfrak{i} i g$, bein.

Sie naht, ben E orberzroeig zu reiden;

Der Lorber ift bes Muthes aeiden,

$23 e m$ fledt' id ihn zum Evorenfranz? -

Dir, ebler Seroz obne Bleidyen!

Du Stab und Scort bes Baterlanbs!

Du reigit bie Ihat an ben Entidglü, 
Bitt Sieger, too Gefahren bräu'n, $\mathfrak{J a}$, bifi ber Bränzen Benitiz, und ficherft $\Re$ tuh und ueberflup. Dein fey bey Aranz, o Felbherr, bein !

23 ie ? - Du , bas nie voran fid) brăngte, Sejheib'nez $\mathfrak{B}$ eild)en? - Bwang nur mengte \$ier unter ftolze $\mathfrak{B l u m e n}$ bid). şilltommen! - Seit ber Sindheit băngte Mein Şerz an beine Frarbe fing. Shr , ftill entblügt uno fdlid)t, wie bu, SBsie bu, fürwabr! veftalijo rein,

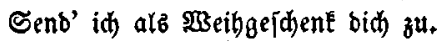
Empfang es mit ber unfhuld $\Re$ th! Dein ift ez, gute $\subseteq$ dwefter, bein!

Shun beut fie mir bie smmortelle, Boll fðönen Ermftez, dá fie Duelle Begeifternber (Bebanken (ey).

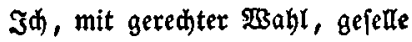
Der Fireunbihaft fie zum Sinnbilb bei. Dir, Jreunb! - Mix weibft ja brübertith 
382

Du bie zum leâten 5audde biđ -

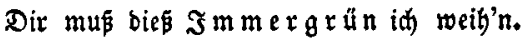

Dein, o mein zreites befireb $\Im$ d)!

Dein $\{e r ' s, \mathfrak{z} r e$ und meiner Secle, bein.

Nun zeigt fie mit veridömten Bliden

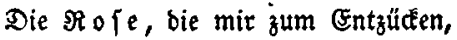

Mit ifrem $B$ ufen ftieg uno fanE.

》) SBsen foll bie Bstüctidthe begtüden ? 》 -

So rief id Enieend, liebetrane.

Cie ftans, ben Finger auf bem Munbe,

Şalb göttltid in ber Sonne Sdyein,

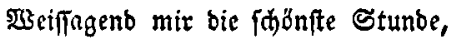

und ftammelte bie Simmelkunde:

》) Rur bein ift meine $\Re$ ofe, bein!

₹r. Sุaug. 


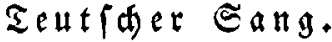

\section{, Ed)Itdte Sort uno gtt Gemút}

Jit bas redite teutidje lied. "

Эoy. ছeinr, Boecler jll Etráburg 3640 .

Lapt das trutfoc lieb exidgallen burch ben reiten Diăterhain, tonreid, wie bie Radtigallen, wie ber $\mathfrak{E} e r d$ e Sänge, rein! Einfad, wie im neuen Eenze für ber Sđåaferinnen Nränze. eine wilbe Rofe brüht! Shlidite ift bas rechte teutjde sieb.

Şą bie innre Stimme fobert wirb in Sängen offenbar, wie in reinen Flammen lobert frommes Spfier vom Zltar! 
384

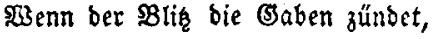
wirb bie Didjterglut verkündet, bie zum Şimmel aufwärţ iprülyt. Sdlidte \$Bort und gut Bemüth ift bas redite teutiche sieb.

Frablingżarten, Wehrenfelber und too Saft ber Reben rinnt, bort ift für bie Dichterwäl(ber 1 )

ein ergöşlid) Eaburinth, wenn fich an bez Spindus Şöhen bolt: Rlarien 2) ergeben, beren Sdlidte s'Bort und gut (semüt) ift bas redite teutfde sieb.

Eolber Frauten Gumft gerwinn

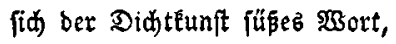
Denn ber Dienft ber keufden Minne

ift DeE Minftrel 3iel und \$ort! Jeber hält in Goben Ehren, des Bejangez alte Eeyren, 
fühlt's, wenn er zum

Sdlidite Wort und gut (Semüth

ift bas redite teutjhe Rieb,

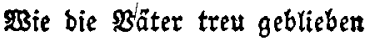

bem verebrten Gängeramt,

fo nod) heut von: $\mathfrak{3}$ औas wir lieben -

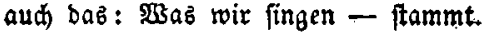

In ben Näben, in ben Fernen,

wo viel bolbe 2ugen fternen, 3)

ift ber $\mathfrak{Y}$ oefie Bebiet!

Sd)lidte \$sjort unb gut Bemuth

ift bas redite teutjdie fieb,

T3o fid billben neue röne, wo erfunden neuer $\mathfrak{B a r}, 4)$ werbe wahr bas Einfadjidäne, benn nur bies ift einfadwahr. Sdjmude unb Zierbe mögen idwinben, wenn wir bas nur wieberfinben, waz in suren innig glüht:

Sdlidte 330 ort und gut Bsmunth

ift bab redite teutione Eieb.

Uxtbur yom Rordtern, 


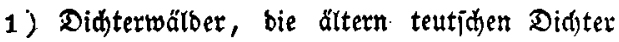
roie z. B. Splis, Flemming lieferten ihre (5ebid)te

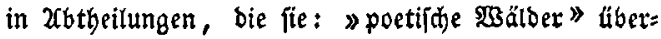
idricben.

2) Rlarien - fo nannten bie ăltern Did)ter bie Mujen, yon Xpoll bem Bott yon Silaroz; (Servius ad Virg. Aes. 3, 360.) 3. $\mathfrak{B}$.

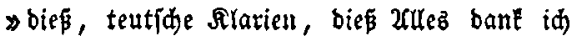
eud!"

$\mathfrak{g} \mathfrak{a} \mathfrak{u} \mathfrak{f} \mathfrak{F} \mathfrak{l} \mathfrak{m} \mathfrak{m} i n g$.

3) fternen, von Stern, altce gutes von Flcm= ming, Eobenftein 26 . gebraud)tez, feitbem unbenuşt gebliebenes 200 t.

4) $\mathfrak{B a r}$, Eieb ber Meifterjänger. 


\section{$\mathfrak{D} \mathfrak{e} \mathfrak{i} \mathfrak{r} \mathfrak{i} \mathfrak{n} \mathfrak{e} \mathfrak{e}$}

\section{$1785,{ }^{*}$ )}

Immer bin, unb immer ber, S(t nur nidjt mein $\mathfrak{B e d j e r}$ leer!

Şa wie flopit mein \$erz vor Freuben, und wie fuminben alle Reiben, खsenn ber $\Re$ ebenjaft mir lart, Dab (Gehirn mir leidfter madt. Simmer hin und immer ber, Sit nur nidht mein $\mathfrak{B}$ ed)er leer.

Stimmer fen mein Bedter leer; Dann mag toben Noro uno Meer!

Mögen Fürften fid zerquälen,

Reidje golone Gdäąe zählen,

Shoren fuben hoben Rang,

(Broper Zitel Leeren Rlang;

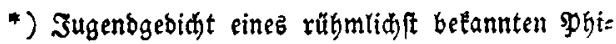
Iologen.

ช. 5 . 
388

Diefes madjt mein Serz nidht fdrwer, Sit nur nid)t mein $\mathfrak{B}_{\text {edjer }}$ leer.

W3ie ift mir bie $\mathfrak{z B}_{3} \mathrm{lt}$ to bebr, Reine Stunbe wonncleer!

Mögen anbre Bseibern fröhnen,

und bor Riebe feufzen, ftöhnen! -

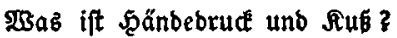

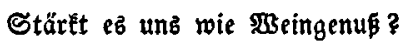

Rein! - Drum geht bez నånbelns leer;

Piur bez ssötterjaftea mebr:

(sebt ihr mir bes Saftes mehr,

$D$ bann hüpf' id bin unb ber.

Mag ber Beiten Eile flüftern,

Daß mid wirb ber aob umbúftern,

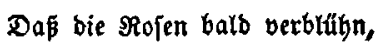

Die um meine Stune grähn: -

$D$ ! beb ad)t' id nimmermehr,

3it nur nidyt mein $B$ eder leer! 